\title{
Interest Rate Pass-Through in the Common Monetary Area of the SACU Countries
}

Citation for published version (APA):

Kleimeier, S., \& Sander, H. (2005). Interest Rate Pass-Through in the Common Monetary Area of the SACU Countries. Finance.

Document status and date:

Published: 01/01/2005

Document Version:

Publisher's PDF, also known as Version of record

\section{Please check the document version of this publication:}

- A submitted manuscript is the version of the article upon submission and before peer-review. There can be important differences between the submitted version and the official published version of record.

People interested in the research are advised to contact the author for the final version of the publication, or visit the DOI to the publisher's website.

- The final author version and the galley proof are versions of the publication after peer review.

- The final published version features the final layout of the paper including the volume, issue and page numbers.

Link to publication

\footnotetext{
General rights rights.

- You may freely distribute the URL identifying the publication in the public portal. please follow below link for the End User Agreement:

www.umlib.nl/taverne-license

Take down policy

If you believe that this document breaches copyright please contact us at:

repository@maastrichtuniversity.nl

providing details and we will investigate your claim.
}

Copyright and moral rights for the publications made accessible in the public portal are retained by the authors and/or other copyright owners and it is a condition of accessing publications that users recognise and abide by the legal requirements associated with these

- Users may download and print one copy of any publication from the public portal for the purpose of private study or research.

- You may not further distribute the material or use it for any profit-making activity or commercial gain

If the publication is distributed under the terms of Article $25 \mathrm{fa}$ of the Dutch Copyright Act, indicated by the "Taverne" license above, 
First Version, August 2004

Comments are welcome

\title{
INTEREST RATE PASS-THROUgh IN AN ENLARgED EUROPE: \\ The Role of Banking Market Structure for Monetary Policy Transmission in TRANSITION COUNTRIES
}

by

\begin{abstract}
Harald Sander ${ }^{\mathrm{a}, \mathrm{c}^{*}}$ and Stefanie Kleimeier ${ }^{\mathrm{b}, \mathrm{c}}$
a. Faculty of Economics and Business Administration, University of Applied Sciences Cologne, Claudiusstr.1, 50678 Köln, Germany.

${ }^{\mathrm{b}}$ Limburg Institute of Financial Economics, Maastricht University, Tongersestraat 53, 6211 LM Maastricht, The Netherlands.

${ }^{c}$. METEOR, Maastricht University, Tongersestraat 53, 6211 LM Maastricht, The Netherlands.
\end{abstract}

\section{Abstract}

This study investigates the transmission of monetary policy onto retail bank interest rates in the eight Central and Eastern European countries (CEECs) that joined the European Union in 2004. Focussing on the period from 1993 to 2003, we employ a unifying empirical passthrough model that allows for thresholds, asymmetric adjustment, and structural changes over time. Our results show that the pass-through in many CEECs is faster and more complete than in the eurozone and that there may be a high potential for convergence of the pass-through across CEECs with market concentration, bank health, foreign bank participation and monetary policy regime as conditioning factors.

JEL Classification Numbers: E43; E52; E58; F36.

Keywords: Interest Rate Pass-Through, Monetary Policy, European Monetary Union, European Enlargement, Transition Economies, European Banking, Competition in Banking, Banking Market Structure, Asymmetric Adjustment.

* Presenting and corresponding author. Tel: +49-221-82753419; fax: +49-221-82753131; E-mail address: gh.sander@t-online.de (Harald Sander) 


\section{$\underline{1 . \text { Introduction }}$}

On May 1, 2004 eight Central and Eastern European Countries (CEECs) joined the European Union (EU). EU-membership implies participation in the single market and the prospect of eurozone membership. For the eurozone incumbents, recent research has shown that the emergence of an integrated European banking market is much slower than expected ${ }^{1}$, mainly due to a lack of cross-border lending and cross-border mergers and acquisitions. However, an integrated banking market is often considered to be a precondition for a smooth, efficient and homogeneous transmission of monetary policy impulses under a single currency. In contrast, in CEECs participation of foreign banks is substantial and may thus play an important role for the eventual emergence of an integrated banking market and a homogeneous monetary transmission process. One essential avenue of investigating these issues is to analyze the pass-through of monetary policy impulses on retail interest rates. Whereas there is ample evidence for the eurozone, this study will be the first to investigate these issues for all eight CEEC accession countries.

With respect to the eurozone most pass-through studies are based on a variant of the pioneering work by Cottarelli and Kourelis (1994). Here important include BIS (1994), Cottarelli, Ferri and Generale (1995), Borio and Fritz (1995), Mojon (2000), de Bondt (2002), de Bondt et al. (2002), Sander and Kleimeier (2002), Toolsema, Sturm and de Haan (2002), and Heinemann and Schüler (2003). Typically these studies find considerable differences in the pass-through not only across different bank lending and deposit rates but also across the countries of the eurozone. Moreover, a substantial degree of short-run bank interest rate stickiness is found. At the same time many but not all studies find evidence for a less than full pass-through in the long-run which can be read as evidence for credit rationing phenomena.

\footnotetext{
${ }^{1}$ A recent overview on financial integration in the eurozone is given by Freixas (2003) and Baele et al. (2004). While the latter study uses predominantly so-called beta- and sigma-convergence measures, Kleimeier and Sander (2000, 2003) and Schüler and Heinemann (2002) investigate retail banking market integration using cointegration methodology. For an application of the cointegration approach to CEECs see Brada, Kutan, and Zhou (2002).
} 
Furthermore, asymmetric adjustment of retail interest rates depending on the type of interest rate shock is regularly documented. Finally, it is often argued that the single currency could act as a unifying force that has the potential to make the pass-through faster, more complete and more homogeneous. Despite these commonalties, the existing studies are often using differing approaches and consequently lead to diverging results. Sander and Kleimeier (2004) therefore introduce a unifying methodological framework for analyzing the pass-through process in the eurozone. The results of these pass-through estimates are then used in secondstage regressions to shed light on the role of competition and integration for a more uniform monetary transmission. For the eurozone, these authors argue that the evidence points to a lack of integration and in particular to the role of legal and cultural differences that may continue to preclude full convergence in the near future even after the introduction of the single currency.

Given the even more diverse structures of the new EU member countries one might hypothesize that enlarging the eurozone will lead to an even more heterogeneous monetary policy transmission process in the EMU. Table 1 provides an overview of important macroeconomic and financial sector developments and characteristics. On the macro-economic side the CEECs have typically experienced higher GDP growth rates and higher inflation rates than the eurozone. However, the reduction of both, inflation and money market rate volatility stands out and might be of high relevance for the pass-through process. The second set of variables relates to the reform and development of the financial sector. A comprehensive assessment of the state of banking sector reform is regularly given by the European Bank of Reconstruction and Development (EBRD), which reports a banking reform index. ${ }^{2}$ While some progress has been made, most CEECs still fall short of eurozone standards which are

\footnotetext{
${ }^{2}$ The assessments are by EBRD country economists. A score of 1 reflects little change except the separation of central banking from commercial banking. A score of 2 can be obtained after significant liberalization of interest rates and credit allocation. For a score of 3 progress has to be made regarding regulation, supervision and resolution of bank insolvencies and establishment of hard budget constraints. A 4+ score reflects a level of reform that approximates advanced industrialized country standards.
} 
reflected by the maximum score of $4+$. This is also true regarding two prominent measures of financial development: As one of the most comprehensive measures of financial development, the ratio of private credit to GDP reveals that despite some progress CEECs still have a long way to go to converge to advanced country levels of financial deepening. The same holds for stock market capitalization. ${ }^{3}$ Some more progress has been made with respect to intermediation margins that are now more in line with industrialized country standards, while at the time the share of non-performing loans could often, but not always be reduced. Nevertheless, its share is still very high. Regarding further important financial structure indicators the picture is heterogeneous. Concentration - here measured as the asset share of the three largest banks in per cent of asset of all commercial banks - is increasing in some cases and decreasing in others, but still conveying substantial differences across countries. In contrast to eurozone banking markets the share of foreign banks is typically very high, with the notable exception of Slovenia and Lithuania. Particularly Slovenia stands out in its reform of the banking system as not only foreign bank entry was restricted for a long time but also the privatization process of banks has for long remained incomplete as can be seen from the still high asset share of state-owned banks in 2002.

(Insert Table 1 about here)

By now there are only very few pass-through studies for CEECs and these are then often limited to individual countries, such as the study by Opiela (1999) and Chmielewski (2004) for the case of Poland, and Crespo-Cuaresma, Égert and Reininger (2004) who compare the Czech Republic, Hungary and Poland. Generally, in all papers short-run stickiness of retail interest is confirmed, while at least in some countries - in particular Poland - strong evidence for a full pass-through is found. The latter authors furthermore find

\footnotetext{
${ }^{3}$ Germany - for example - has a credit to GDP ratio of about 120 per cent. Stock market capitalization in 2002
} 
evidence for a heterogeneous pass-through process across market interest rates and across the three countries.

Our study will extend these works by applying the Sander-Kleimeier approach to all eight new CEECs. The results of our study will show that the pass-through process in CEECs is also characterized by short-term price stickiness, but that it is generally more complete and less asymmetric than in the eurozone. Moreover, we confirm that there is heterogeneity across the eight CEECs, but much less than in the existing eurozone. However, this heterogeneity can largely be explained by a few variables describing macro-economic conditions and financial structure. Thus, conditional convergence can be predicted with market concentration, bank health (as measured by the share of bad loans), foreign bank participation and the monetary policy regime as conditioning factors. With respect to the latter, one might therefore expect that after joining EMU, the newcomers may constitute a quite homogeneous monetary transmission region - which may stand in contrast to the much more divergent picture that can be obtained from the incumbents.

\section{Data and Methodology}

Our study focuses on the Czech Republic, Estonia, Hungary, Latvia, Lithuania, Poland, the Slovak Republic and Slovenia. For these countries we collect monthly data from January 1993 to December 2003 for eight retail interest rates on mortgages, consumer loans, short- and long-term corporate loans, current account deposits, time deposits and savings accounts. As a proxy for the central bank's policy rate we use a one-month money market rate. Our sources for these data are the web-sites of the national central banks. Only in cases where the central bank does not report any money market rate, the series is obtained from the IMF International Financial Statistics. Overall, our sample contains 108 different retail interest rates comprising 32 different loan rates and 76 different deposit rates. Note, however, 
that the majority (69 of 76) of the deposit rates belongs to the category of time deposits. To analyze and account for any structural changes in the national banking markets that might have occurred since 1993, we decide to conduct our analysis for five-year rolling sub-periods and consequently obtain results for 1993-1997, 1994-1998, and so on until 1999-2003. This leads to a total sample of 358 observations of which 102 are loan observations and 256 are deposit observations. Details on the different national rates, their exact definitions and availability are given in Table A1 of appendix A. The development of these rates over time is presented in Figure A1 in the same appendix.

Following Kleimeier and Sander (2004), we model the empirical pass-through analysis as a unifying approach that utilizes VAR and cointegration methodologies and that allows for asymmetric and threshold adjustment. Our pass-through model can take one of the three basic forms:

$$
\begin{aligned}
& \mathrm{BR}_{\mathrm{t}}=\beta_{0}+\sum_{\mathrm{i}=1}^{\mathrm{k}^{*}} \beta_{\mathrm{BR}, \mathrm{i}} \mathrm{BR}_{\mathrm{t}-\mathrm{i}}+\beta_{1} \mathrm{M}_{\mathrm{t}}+\sum_{\mathrm{i}=1}^{\mathrm{n} *} \beta_{\mathrm{M}, \mathrm{i}} \mathrm{M}_{\mathrm{t}-\mathrm{i}}+\varepsilon_{\mathrm{t}} \\
& \Delta \mathrm{BR}_{\mathrm{t}}=\sum_{\mathrm{i}=1}^{\mathrm{k}^{*}} \beta_{\mathrm{BR}, \mathrm{i}} \Delta \mathrm{BR}_{\mathrm{t}-\mathrm{i}}+\beta_{1} \Delta \mathrm{M}_{\mathrm{t}}+\sum_{\mathrm{i}=1}^{\mathrm{n}^{*}} \beta_{\mathrm{M}, \mathrm{i}} \Delta \mathrm{M}_{\mathrm{t}-\mathrm{i}}+\varepsilon_{\mathrm{t}} \\
& \Delta \mathrm{BR}_{\mathrm{t}}=\sum_{\mathrm{i}=1}^{\mathrm{k}^{*}} \beta_{\mathrm{BR}, \mathrm{i}} \Delta \mathrm{BR}_{\mathrm{t}-\mathrm{i}}+\beta_{1} \Delta \mathrm{M}_{\mathrm{t}}+\sum_{\mathrm{i}=1}^{\mathrm{n} *} \beta_{\mathrm{M}, \mathrm{i}} \Delta \mathrm{M}_{\mathrm{t}-\mathrm{i}}+\beta_{\mathrm{ECT}} \mathrm{ECT}_{\mathrm{t}-1}+\varepsilon_{\mathrm{t}}
\end{aligned}
$$

where $\mathrm{BR}_{\mathrm{t}}$ and $\mathrm{M}_{\mathrm{t}}$ are retail and money market rates, respectively, and $\mathrm{k}^{*}$ and $\mathrm{n}^{*}$ indicate the optimal lag lengths. ${ }^{4}$ Equation (1) describes the pass-through model as a standard VAR in levels (STD_LL) and is chosen for interest rates that exhibit an $\mathrm{I}(0)$ property. However, since interest rates are often found to be I(1), the empirical pass-through model is best estimated using first differences as stated in equation (2). This standard VAR specification (STD) avoids spurious regression problems but leads to a loss of information about long-run relationships. If $\mathrm{BR}$ and $\mathrm{M}$ are cointegrated, this information can fortunately be recovered.

\footnotetext{
${ }^{4}$ Whenever an optimal lag length has to be determined, the minimum AIC criterion is used allowing for a maximum of four lags.
} 
The VAR then needs to be augmented by a lagged error correction term $\left(\mathrm{ECT}_{\mathrm{t}-1}\right)$ as shown in equation (3). By estimating the cointegration relationship (4) the ECT can be obtained as shown in equation (5): ${ }^{5}$

$$
\begin{aligned}
& \mathrm{BR}_{\mathrm{t}}=\theta_{0}+\theta \mathrm{M}_{\mathrm{t}}+\mathrm{u}_{\mathrm{t}} \\
& \mathrm{ECT}_{\mathrm{t}-1}=\mathrm{u}_{\mathrm{t}-1}
\end{aligned}
$$

The pass-through process is often characterized by threshold and asymmetric adjustment mechanisms, at least in the existing eurozone (Sander and Kleimeier, 2004). Next to the symmetric error-correction process we therefore consider several asymmetric specifications for the adjustment of interest rates. For all models, we define the long-run cointegrating relationship as given in equation (4). The symmetric model (SYM) visualizes immediate adjustment, which is independent of the state of the dis-equilibrium. Thus, the ECT is defined as in equation (5). The asymmetric adjustment models all belong to the group of threshold autoregressive (TAR) models. They differ with respect to the definition of the ECT and can model different type of adjustment processes. Consider a situation where out of an equilibrium, changes in the money market rate are followed by changes in the retail bank rate. While the SYM model allows only for the same speed of adjustment regardless of the size and direction of the shock, the $\mathrm{TAR}^{0}$ model allows for a different adjustment speed to rising versus falling money market rates. Thus, the threshold is implicitly set to zero. The second asymmetric model (TAR*) is a modification of the $\mathrm{TAR}^{0}$ such that the threshold is now allowed to deviate from zero. The rationale is that retail rates may adjust differently to a disequilibrium once a certain minimum deviation in one direction is exceeded. The third variation is a Band-TAR model (B-TAR*) which can reflect both interest rate smoothing as well as interest rate stickiness driven by menu-cost behavior of banks. Menu-cost behavior could, for example, be relevant if we find cointegration only outside a band, which is defined

\footnotetext{
${ }^{5}$ Cointegration testing is based on the Durbin-Watson (DW), Dickey-Fuller (DF) and augmented Dickey-Fuller (ADF) tests. The details of these tests and all other methodology can be found in appendix C.
} 
by a symmetric threshold around zero. Finally, our fourth and fifth asymmetric models represent momentum threshold autoregressive models $\left(M-T A R^{0}, M-T A R^{*}\right)$ where the adjustment depends on the speed of deviation from equilibrium. Therefore, M-TAR adjustment can reflect behavior by banks, which attempt to smooth out large market rate changes.

(Insert Figure 1 about here)

In order to find the empirical pass-through model that optimally fits the data we follow an (almost) automatic model selection procedure that is illustrated in Figure 1. We start with unit root testing of each interest rate for the full sample period. In case of $\mathrm{I}(0)$ for both BR and M we choose the STD_LL model as the optimal pass-through model. ${ }^{6}$ Otherwise, we proceed with cointegration testing. We first estimate all TAR-type models and select that TAR-type model which best fits the data based on the AIC criterion. For this model, we conduct cointegration and asymmetry tests. If asymmetric cointegration is confirmed, the pass-through model is set as the best TAR-type model. If asymmetric cointegration is not confirmed, we continue with symmetric cointegration testing. If symmetric cointegration is found, the passthrough model is set as SYM. If symmetric cointegration is rejected, the select the standard model (STD). We conduct this selection process for all rolling sub-periods of five years each. Since it is our objective to select for each national retail rate the same pass-through model for all sub-periods, we need a decision rule to select one and only one pass-through model. Our first rule is to choose the model that occurs in the absolute majority of all rolling sub-periods. If no model has the absolute majority, we repeat the estimation and selection procedure for

\footnotetext{
${ }^{6}$ The results of the unit root tests are shown in Table B1 of appendix B. For all Slovenian rates, we choose the STD_LL model. For all other national rates the I(1) property can be confirmed.
} 
the full sample period and choose this model also for all sub-periods. ${ }^{7}$ Based on the selected pass-through models, multipliers of different time horizons (impact, 1, 3, 6, 12 months, and long run) are obtained for positive and negative interest rate shocks of $1.00 \%$ and $0.25 \%$.

Based on these multipliers we try to identify which variables determine the passthrough by means of running second-stage OLS regressions. In a first step we explore country and market differences as well as structural changes over time. To do so we simply regress our multipliers on countries, market, and time dummies. ${ }^{8}$ Additionally we use dummies for different types of monetary policy shocks to explore the existence and nature of potential asymmetries. In a second step we attempt to replace these dummies by macro-economic control variables and financial structure variables in order to provide an as parsimonious model as possible. After some experimenting with different estimations we have settled for two macro-controls ${ }^{9}$ and four financial market descriptors ${ }^{10}$. Our first macro-economic control variable is the volatility of the money market rate $(m m v o l)$, defined as the standard deviation of the monthly money market rates for the respective rolling period and country under consideration. The second one, inflation, is defined as the percentage change on the previous year's average annual harmonized index of consumer prices (European Commission, 2004: Table 35).

These data for three of our four financial structure variables are obtained as annual observations from the EBRD Transition Reports for 2002 and 2003. It should be noted that these are often highly correlated. Therefore we first tried the EBRD's comprehensive measure

\footnotetext{
${ }^{7}$ Estimation results for the full period and rolling sub-periods are listed in tables B2 to B5 in appendix B. Since in some cases we impose the full-period model on the sub-periods, we are following a conservative strategy in selecting asymmetric models for the full period. More specifically, we accept the existence of asymmetric cointegration at the $10 \%$ significance level in the rolling sub-periods but only at the $1 \%$ level in the full period.

${ }^{8} \mathrm{We}$ are grateful to Robert DeYoung for suggesting this regression framework to us for an earlier study.

${ }^{9}$ We also collect data regarding real GDP growth from Table 1 of European Commission (2004) and domestic credit to enterprises (in \% of GDP) from the World Banks Financial Development and Structure Database (Revised: October 28, 2003). As discussed in section 4, we find, however, that these two macro-controls do not contribute to the explanatory power of our model.

${ }^{10}$ Regarding financial structure descriptors we have collected a large number of variables regarding competition, foreign banks participations, bank health, capital market development, the state of the bank and financial reform
} 
of banking sector reform (bankreform), which ranges from 1 to $4+$ with a higher index number reflecting further-reaching reforms. To obtain a measure of foreign competition we employ the EBRD's number for domestic and foreign-owned banks. We construct frgbankp as the number of foreign banks as per cent of all banks. ${ }^{11}$ As a measure of bank health we use badloan defined as non-performing loans in per cent of total loans. ${ }^{12}$ Finally, our fourth proxy CR3 measures the annual concentration of the national banking market and is obtained from the World Bank (2003) Financial Development and Structure Database. CR3 is defined here as assets of the three largest banks in per cent of assets of all commercial banks in the country. ${ }^{13}$ To correspond to our five-year rolling samples, all annual structural variables are converted to five-year averages.

\section{The Pass-Through in CEECs}

From our pass-through regressions we obtain multipliers for all countries, retail interest rates and seven different overlapping periods leading to a total of 358 usable observations. At a first inspection of the results it appears that the pass-through has become somewhat more complete and somewhat faster. ${ }^{14}$ Figure 2 illustrates this impression by showing the average multipliers for various retail banking markets. It can immediately be seen that the pass-through is most efficient with respect to lending to the corporate sector and least efficient with respect to current account and savings deposits. Moreover, over time the changes in the pass-through exhibit a V-shaped adjustment, i.e. the impact and very short-run multipliers have decreased over time, while long(er)-run multipliers have increased over time.

\footnotetext{
process, etc. from the EBRD's transition reports as well as from the World Banks Financial Development and Structure Database (Revised: October 28, 2003).

${ }^{11}$ The EBRD defines "Number of banks (foreign-owned)" as: "Number of commercial and savings banks, excluding cooperative banks. Foreign-owned banks are defined as those with foreign ownership exceeding a 50 per cent share, end-of-year".

${ }^{12}$ The EBRD variable "Non-performing loans (in per cent of total loans)" is defined as: "Ratio of nonperforming loans to total loans. Non-performing loans include substandard, doubtful and loss classification categories for loans, but excludes loans transferred to a state rehabilitation agency or consolidation bank, end-ofyear."

${ }^{13}$ The World Bank obtains these data from the Fitch Bankscope Database.
} 
These impressions could, however, be misleading as we obtain them by simply averaging over all available data. Thus, if some countries start reporting earlier and in much more detail than others, country differences can introduce a bias into the reported averages. Therefore, we use the panel regression approach described in the previous chapter to test for pass-through differences across countries, markets and sub-periods. Here we regress all multipliers of a specific time horizon (h) on dummies for country- (c), market- (m), and time- (t) specific effect as indicated in equation (6):

$$
\begin{array}{r}
\mathrm{h}-\text { multiplier }_{\mathrm{c}, \mathrm{m}, \mathrm{t}}=\alpha_{0}+\sum_{c=1}^{C} \alpha_{c} * c_{-} \text {dummies }_{c}+\sum_{\mathrm{m}=1}^{\mathrm{M}} \alpha_{\mathrm{m}} * m_{-} \text {dummies }_{m} \\
+\sum_{\mathrm{t}=1}^{\mathrm{T}} \alpha_{\mathrm{t}} * t_{-} \text {dummies }_{t}+\varepsilon_{\mathrm{c}, \mathrm{m}, \mathrm{t}}
\end{array}
$$

These second-stage regressions are reported in Table 2 and yield important insights. Regarding the long-run multipliers which reflect the size of the pass-through, the country dummies are all significant and negative except for Slovenia where the size of the passthrough is larger, and Hungary which seems to share the long-run multipliers with the benchmark country Poland. Whereas country differences are marked, the long-term reaction to monetary policy changes is not differing across markets. Exceptions are - not unexpectedly - current and saving account rates which display a less complete pass-through. Finally, the time dummies provide clear evidence for a development towards a full pass-through over time, which may reflect the development of the financial sector in CEEC towards a more competitive banking system.

(Insert Figure 2 about here)

(Insert Table 2 about here)

\footnotetext{
${ }^{14}$ The details on all multipliers can be obtained from Table B6 in appendix B.
} 
How fast countries and markets approach these long-term equilibria can be seen from the regressions for the impact and interim multipliers, which reflect the speed of the passthrough. Here, country differences are not so strong in the very short horizon (impact to 1month multipliers) but are becoming more relevant as the long-run equilibria are approached. Regarding different markets, interest rates charged for lending to the corporate sector, both short-term and long-term, are reacting significantly faster while current account and saving rates are reacting significantly slower.

As, however, the dummies for the rolling regressions indicate, impact multipliers become smaller over time. But as interim multipliers have increased in the recent years, passthrough speed has accelerated and monetary transmission has improved in efficiency. This Vshaped development that could already be observed in Figure 2 is therefore confirmed here. A possible explanation for this pattern could be the decreased volatility in money markets in the recent years which reduces the necessity to fast and instantaneous adjustment but at the same time allows a more complete adjustment in the long-run - an effect that will be supported later by our structural regressions.

In a second step we take into account the differences in loan and deposit markets by splitting the sample into two separate panels. ${ }^{15}$ Panel B in Table 2 reveals that the long-run pass-through in the loan market has less country differences than the general pass-through. There are also no significant market differences and generally the pass-through has improved in the long run. More often than not the loan markets are characterized by a full pass-through. While details on different markets and countries can be obtained from Table B6 in the appendix, it should be noted here that average long-run multipliers are 0.91 for short-term corporate and 1.07 for long-term corporate loan rates. For consumer loans the average is 0.59 ,

\footnotetext{
${ }^{15}$ When trying to explain market characteristics by variables describing financial market structures one can show - not unexpectedly - that these variables have different effects in loan and deposit markets. These can be captured by multiplying the structural variables with a loan or deposit market dummy (see Sander and Kleimeier, 2004), respectively, or by splitting the sample into two separate panels. Since we do have ample observations due to our rolling regression approach we opt here for the second alternative.
} 
but the insignificant consumer loan dummy in our panel regression is caused by a high standard deviation for consumer loan multipliers, thus revealing a high degree of heterogeneity in the consumer credit products and the corresponding data. ${ }^{16}$ Rather than in the size, differences are more pronounced in the speed of the pass-through. Particularly, speed is higher in corporate loan markets. The V-shaped change of the pass-through process is largely confirmed for the loan market. Finally one can see from Panel $\mathrm{C}$ in Table 2 that the developments in the deposit markets are by and large in line with the ones found in the general panel. It should however be highlighted that there is much more variation in the deposit panel than in the loan panel thus pointing to more heterogeneity within and across national deposit markets. Finally, one can observe from Table 2 that the multipliers are very similar independent of the size or direction of the monetary policy shock. For an explicit test we also constructed a large panel using all multipliers for all types of shocks. We then apply the standard dummy regression framework for the general and the separate loan and deposit panels to test for adjustment differences depending on the nature of the shock. Table B7 in appendix B shows that these differences are not statistically significant. This result is not surprising since we select an asymmetric model in only about one quarter of all cases. This is partly due to the fact that the model selection procedure indeed rejects asymmetry, but is also influenced - at least partly - by our decision to use only one model per country and market. Nevertheless, the lack of asymmetry is also confirmed by Crespo-Cuaresma, Égert and Reininger (2004) for the Czech Republic, Hungary and Poland and by Chmielewski (2004) for Poland.

In sum, we find a quiet homogeneous pass-through in CEECs. Moreover, especially in lending markets a full pass-through can often be observed, suggesting that credit-rationing phenomena are largely absent. Both results are standing in some contrast to the more heterogeneous and less perfect pass-through in the eurozone (Sander and Kleimeier, 2004).

\footnotetext{
${ }^{16}$ Chmielewski (2004) also finds similarly high long-run multipliers for Polish corporate loan rates and a smaller
} 
Furthermore, the differences in deposit and lending markets suggest to continue any further analysis with separate loan and deposit market samples and in the latter case - given the negative values for current account and savings rates dummies - a separate panel for time deposits. Finally, the V-shape time pattern of the adjustment of multipliers suggests that the monetary regime (as measured by money market rate volatility), macro-economic developments, financial reform and changes in the financial structure may have played an important role in changing the monetary transmission process in CEECs. These pass-through determinants are explored in more detail in the following.

\section{Structural Determinant of the Pass-Through in CEECs}

To explore the role of structural determinants we collect a large number of variables describing the (evolution of) the financial structure and macro-economic developments. We use these variables in second-stage regressions separated for loan, deposit, and time deposit markets. This allows us not only to identify the most important structural determinants but also to investigate whether and to what extent country or market characteristics are precluding full (conditional) convergence across CEECs.

To control for differing macroeconomics conditions we have initially included GDP growth, inflation, financial development (as measured by the ratio of credit to GDP) and money market rate volatility. The financial development variable, however, is not systematically explaining the pass-through pattern while at the same time introducing multicollinearity problems. GDP growth is found to be insignificant in all regressions and the goodness of fit is typically higher without this variable. For the subsequent analysis we have therefore opted to exclude both macro-controls. When investigating the role of inflation in an approach using macro-controls only, we find that higher inflation leads to a somewhat higher speed at later stages of the pass-through process and will also positively affect the long-run 
multiplier. These effects are somewhat weaker when introducing financial market structure variables, but we find them important enough to include inflation even in a very parsimonious approach. A special role is played by money market rate volatility (mmvol). Studies have shown that money market volatility is positively correlated with interest rate margins (e.g. Saunders and Schumacher, 2000) and negatively correlated with the pass-through (Mojon, 2000; Sander and Kleimeier, 2004). Here we can confirm these results as we find that a lower volatility has a positive impact both on the size as well as the speed of the pass-through after some 6 months. However, the impact and early interim multipliers show the opposite result. This may reflect the need to adjust loan rates somewhat faster under a more unstable regime in the very short-term, while in the longer-term this regime would in fact slow down the transmission, reduce the pass-through and thus eventually lead to higher intermediation margins. This effect is confirmed independent of the specification of financial structure variables chosen in the subsequently discussed regressions, which also contain alternative specifications regarding financial structure descriptors. As the latter variables have different effects in loan and deposit markets we analyze their role in separate panels (see Table 3).

(Insert Table 3 about here)

Starting with the loan markets, we have first experimented with the bankreform indicator in various specifications of our panel regression, with and without including further financial structure variables. However, this variable does not appear to be significant in any specification for the loan market multipliers and may introduce multicollinearity problems when used jointly with other financial structure variables. We therefore opted to drop this variable in favor of other financial structure variables. After some more experimenting we have arrived at a first very parsimonious specification which includes a measure of banking market concentration (CR3) and a measure of bank health (badloan). Both variables have the 
expected sign, are statistically significant and help to explain around 50 per cent of the loan market multiplier variations in CEECs. A special role that warrants further investigation is played by foreign bank participation (frgbankp) which carries an implausible negative and significant coefficient.

In a second step we have explored whether or not there is still a significant role for market and country characteristics. Regarding market characteristics we find again a faster and more complete pass-through in the corporate loan market in all specifications. Regarding country characteristics only the Slovenia dummy shows a consistent and statistically significant pattern. The pass-through in Slovenia is faster and more complete in all specifications. As could be seen from Table 1 however, in Slovenia the asset-share of stateowned banks remained extraordinarily high with over 40 per cent for the whole sample period. At the same time its share of foreign bank participation of about 11 per cent is very low by CEEC standards. The former fact may generally explain the special role of Slovenia. ${ }^{17}$ Regarding the second fact, we hypothesize that the counterintuitive result regarding foreign competition may also largely be due to the inclusion of Slovenia. In fact, this result disappears when a Slovenia dummy is introduced. Our preferred specification of the loan market multiplier determinants is given in the last 6 columns of Panel A in Table 3. Here we show that foreign participation leads to a faster pass-through process once we treat Slovenia as an outlier (though the size is not affected as there is strong evidence for a full pass-through, obviously dependent on the degree of competition and bank health). Nevertheless, even then the Slovenia dummy, but only the Slovenia dummy remains significantly positive. Finally, all time dummies are insignificant in this specification and are consequently dropped. The results therefore suggest "conditional convergence": If concentration, bank health and the monetary

\footnotetext{
${ }^{17}$ Opiela (1999: 5) argues that the central bank may play the role of an oligopolistic leader and thus may try to "...reduce loan rate stickiness through signaling a desire for loan rates to change by altering the administrative rate. This practice is not only widespread, but may also have more relevance in an economy dominated by stateowned banks whose managers have been used to following administrative orders rather than subtle market signals."
} 
policy regime will converge so will also the financial part of the monetary transmission process in CEECs. This conditional convergence feature distinguishes the new EU members ${ }^{18}$ and potential eurozone newcomers from the much more heterogeneous incumbents.

Turning to deposit markets (Table 3, Panel B) it may first be interesting to see that the EBRD's banking reform indicator has some explanatory power here, although - eventually because of its composite nature - it does not explain much of the variation in the data. After experimenting with a number of specifications, the one reached for the loan market is again the most appealing here. Less concentration, less bad loans and more foreign participation (in all countries but Slovenia) lead to a faster pass-through. Also here the Slovenia dummy remains positive and significant. Money market rate volatility has a special impact here. The higher it is, the faster the pass-through in the very short run - but it has little or no impact on the longer- run multipliers or even on the completeness of the pass-through. Likewise, higher inflation leads to a faster pass-through only in the interim period. Thus a more stable monetary policy regime with low inflation has the potential to slow down the pass-through in the medium term.

Since we find current account and saving account rates reacting much slower than time deposit rates and given the large amount of data on time deposit rates we also run a separate pass-through regression for time deposits. Panel $\mathrm{C}$ in Table 3 reveals that most results of the deposit regressions can be confirmed. But additionally we can show that the pass-through is faster for short maturities and time deposits from the corporate sector. In the latter case the pass-through is also more complete.

\section{$\underline{\text { 5. Conclusions }}$}

The messages from our analysis are straightforward: First, most country differences in CEECs can be explained by a handful of financial structure variables and macro-controls.

\footnotetext{
${ }^{18}$ For future studies it would be interesting to investigate the role of Slovenia as privatization and opening up to
} 
Macro-economic and financial structure convergence can thus be expected to "produce" monetary transmission convergence. This result contrasts sharply to the pattern found in the eurozone countries, where "legal and cultural differences may continue to preclude full convergence" (Sander and Kleimeier, 2004: 490). Secondly, a more competitive market structure, a healthier banking system and higher foreign bank participation play an important role in CEECs to speed up the pass-through. Third, we find that the markets for corporate loans are reacting both faster and more completely to monetary policy impulses - a result that is also confirmed by most pass-through studies. Given that we can often observe a full passthrough, the evidence points to much less occurrence of credit rationing phenomena than in the current eurozone member countries.

To put it in a nutshell, the pass-through process in many CEECs appears to be faster and more complete than in most countries of the eurozone and there may be a high potential for an emerging homogeneous transmission process across CEECs. Convergence can be predicted with market concentration, bank health, foreign bank participation and monetary policy regime as conditioning factors. With respect to the latter, one can expect that after joining EMU, the newcomers may constitute a quite homogeneous monetary transmission region - which may stand in contrast to the much more divergent picture that can be obtained from the incumbents. The issue for the future seems therefore not so much to address heterogeneity across CEECs but vis-à-vis the current eurozone.

foreign banks will accelerate now after EU entry. 


\section{$\underline{\text { References }}$}

Baele, L., Ferrando, A., Hördahl, P., Krylova, E., Monnet, C., 2004. Measuring financial integration in the euro area. European Central Bank Occasional Paper, No.14, April, Frankfurt.

Bank for International Settlements, 1994. National differences in interest rate transmission. CB Document 393, Basle.

Bondt, G. de, 2002. Retail bank interest pass-through: New evidence at the euro area level. ECB Working Paper No. 136, Frankfurt.

Bondt, G. de, Mojon, B., Valla, N., 2002. Interest rate setting by universal banks and the monetary policy transmission mechanism in the euro area. Unpublished working paper, European Central Bank, Frankfurt.

Borio, C.E.V., Fritz, W., 1995. The response of short-term bank lending rates to policy rates: A cross-country perspective. In: Bank for International Settlements, Financial structure and the monetary transmission mechanism. CB Document 394, Basle, pp. 106-153.

Brada, J.C., Kutan, A.M., Zhou, S., 2002. Real and monetary convergence within the European Union and between the European Union and candidate countries: A rolling cointegration approach. William Davidson Working Paper 458. Michigan Business School, April.

Chan, K.S., 1993. Consistency and limiting distribution of the least squares estimator of a threshold autoregressive model. The Annals of Statistics 21, 520-533.

Chmielewski, T., 2004. Interest rate pass-through in the Polish banking sector and bankspecific financial disturbances. Paper presented on the ECB Workshop "Asset Prices and Monetary Policy", December 11-12, 2003, Frankfurt (paper obtained from ECB web-site, Version: January 31, 2004).

Cottarelli, C., Ferri, G., Generale, A., 1995. Bank lending rates and financial structure in Italy: a case study. IMF Working Paper 95/38, Washington, DC. 
Cottarelli, C., Kourelis, A., 1994. Financial structure, bank lending rates, and the transmission mechanism of monetary policy. IMF Staff Papers 41 (4), Washington, DC.

Crespo-Cuaresma, J., Égert, B., Reininger Th., 2004. Interest rate pass-through in the new EU member states: The case of the Czech Republic, Hungary and Poland. William Davidson Institute Working Paper Number 671, Michigan Business School, May.

Enders, W. and P.I. Siklos, 2000. Cointegration and threshold adjustment. Unpublished working paper, Iowa State University, Ames, IA.

Engle, R.F. and C.W.J. Granger, 1987. Co-integration and error correction: Representation, estimation, and testing. Econometrica 55, 251-276.

European Bank for Reconstruction and Development, 2002. Transition Report, London.

European Bank for Reconstruction and Development, 2003. Transition Report, London.

European Commission, 2004. EC economic data pocket book - Quarterly. Office for Official Publications of the European Communities, Luxembourg.

Freixas, X., 2003. European banking integration: Where do we stand? Deutsche Bank Lecture presented on Thursday, 5 June, Saïd Business School, Oxford.

Heinemann, F., Schüler, M., 2003. Integration benefits on EU retail credit markets - Evidence from interest rate pass-through. In: Cecchini, P., The incomplete European market for financial services. Springer Verlag, Berlin, pp. 105-129.

Kleimeier, S., Sander, H., 2000. Regionalisation versus globalisation in European financial market integration: Evidence from co-integration analysis. Journal of Banking and Finance 24, 1005-1043.

Kleimeier, S., Sander, H., 2003. European financial market integration: Evidence on the emergence of a single eurozone retail banking market. Research in Banking and Finance 3, 13-91.

Mojon, B., 2000. Financial structure and the interest channel of the ECB monetary policy. ECB Working Paper No. 40, Frankfurt. 
Opiela, T.P., 1999. The responsiveness of loan rates to monetary policy in Poland: The effects of bank structure. National Bank of Poland, Material and Studies No.17, December, Warsaw.

Sander, H. and S. Kleimeier, 2004. Convergence in euro-zone retail banking? What interest rate pass-though tells us about monetary policy transmission, competition and integration. Journal of International Money and Finance 23, 461-492.

Sander, H., Kleimeier, S., 2002. Asymmetric adjustment of commercial bank interest rates in the Euro area: An empirical investigation into interest rate pass-through. Kredit und Kapital 35 (2), 161-192.

Saunders, A., Schumacher, L., 2000. The determinants of bank interest rate margins: an international study. Journal of International Money and Finance 19 (6), 813-832.

Schüler, M., Heinemann, F., 2002. How integrated are the European retail financial markets? A cointegration analysis. Deutsche Bank Research Notes No. 3, Deutsche Bank, Frankfurt.

Tong, H., 1983. Threshold models in non-linear time series. Lecture Notes in Statistics 21, Springer Verlag, Berlin.

Toolsema, L.A., Sturm, J.-E., de Haan, J., 2002. Convergence of monetary transmission in EU: New evidence. CESifo Working Paper 465, Munich.

World Bank, 2003. World Bank Financial Development and Structure Database. Revised: October 28, 2003. Downloaded from www.worldbank.org. 
Appendix A - Data Description

(insert Table A1 and Figure A1 here)

$\underline{\text { Appendix B - Detailed Estimation Results }}$

(insert Tables B1 to B7 here) 
Appendix C - Details on the Methodology 19

\section{$\underline{\text { Unit Root Testing }}$}

In order to establish whether the interest rates are unit roots, or I(1), two test statistics, a t-statistic and an F-statistic, are employed based on regressions on levels as well as first differences of the underlying series. Both include next to lagged observations of the interest rate $\mathrm{I}$ in question also a trend variable $\mathrm{T}$ :

$$
\begin{aligned}
& \Delta \mathrm{I}_{\mathrm{t}}=\eta_{0}+\eta_{1} \mathrm{I}_{\mathrm{t}-1}+\eta_{2} \Delta \mathrm{I}_{\mathrm{t}-1}+\eta_{3} \mathrm{~T}+\varepsilon_{\mathrm{t}} \\
& \Delta^{2} \mathrm{I}_{\mathrm{t}}=\eta_{0}+\eta_{1} \Delta \mathrm{I}_{\mathrm{t}-1}+\eta_{2} \Delta^{2} \mathrm{I}_{\mathrm{t}-1}+\eta_{3} \mathrm{~T}+\varepsilon_{\mathrm{t}}
\end{aligned}
$$

The null hypothesis states that the series I follows a random walk. This corresponds to a null hypothesis of $\mathrm{H}_{0}: \eta_{1}=0$ for the t-statistic and $\mathrm{H}_{0}: \eta_{1}=\eta_{3}=0$ for the F-statistic. We fail to reject the null hypothesis of a random walk if the calculated $t$ or $F$ values are smaller in absolute terms than the critical values. Thus, as a precondition for cointegration, we have to accept the null hypotheses for equation (C1) but reject them for equation (C2). In particular, we do not proceed with cointegration testing only if both the national retail rate BR and the corresponding money market rate $\mathrm{M}$ are found to be $\mathrm{I}(0)$ at the $5 \%$ significance level.

\section{Symmetric Cointegration and the Pass-Through Model}

The cointegration regression is defined as

$$
\mathrm{BR}_{\mathrm{t}}=\theta_{0}+\theta \mathrm{M}_{\mathrm{t}}+\mathrm{u}_{\mathrm{t}}
$$

A first cointegration testing procedure relies on the Durbin-Watson statistics (DW). The null hypothesis of no cointegration can be rejected when the calculated DW values resulting from the regression of equation (4) are larger than the critical values. As Engle and Granger (1987) point out, the Durbin-Watson test can be used as a good but only approximate indicator for

\footnotetext{
${ }^{19}$ This appendix draws heavily on Sander and Kleimeier (2004).
} 
cointegration and should be followed by a more specific testing procedure such as the DickeyFuller (DF) and augmented Dickey-Fuller (ADF) tests. The Dickey-Fuller test is based on the estimated residuals of the cointegration regression

$$
\Delta \mathrm{u}_{\mathrm{t}}=-\delta_{0} \mathrm{u}_{\mathrm{t}-1}+\varepsilon_{\mathrm{t}}
$$

where the t-statistic for the estimated coefficient $-\delta_{0}$ provides an indication regarding the cointegration of the two series. In particular, the null hypothesis of no cointegration can be rejected when the t-statistic is larger in absolute value than the critical value.

The augmented Dickey-Fuller test is obtained in a two-step procedure from the regression

$$
\Delta \mathrm{u}_{\mathrm{t}}=-\delta_{0} \mathrm{u}_{\mathrm{t}-1}+\sum_{i=1}^{4} \delta_{\mathrm{i}} \Delta \mathrm{u}_{\mathrm{t}-\mathrm{i}}+\varepsilon_{\mathrm{t}}
$$

In the first step equation (C5) is estimated including all 4 lags of $\Delta u_{t-i}$. In the second step, equation (C5) is re-estimated including only the significant lags of $\Delta \mathrm{u}_{\mathrm{t}-\mathrm{i}}$ from step 1 . Now, the null hypothesis of no cointegration can be rejected when the t-statistic for the estimated coefficient $-\delta_{0}$ is larger in absolute value than the critical value.

In case of symmetric cointegration, the pass-through model (SYM) is defined as

$$
\Delta \mathrm{BR}_{\mathrm{t}}=\sum_{\mathrm{i}=1}^{\mathrm{k}^{*}} \beta_{\mathrm{BR}, \mathrm{i}} \Delta \mathrm{BR}_{\mathrm{t}-\mathrm{i}}+\beta_{1} \Delta \mathrm{M}_{\mathrm{t}}+\sum_{\mathrm{i}=1}^{\mathrm{n}^{*}} \beta_{\mathrm{M}, \mathrm{i}} \Delta \mathrm{M}_{\mathrm{t}-\mathrm{i}}+\beta_{\mathrm{ECT}} \mathrm{ECT}_{\mathrm{t}-1}+\varepsilon_{\mathrm{t}}
$$

with the ECT defined as the estimated error term of equation (C6):

$$
\mathrm{ECT}_{\mathrm{t}-1}=\mathrm{u}_{\mathrm{t}-1}
$$

\section{$\underline{\text { Asymmetric Cointegration and the Pass-Through Model }}$}

The threshold autoregressive model $\left(\mathrm{TAR}^{0}\right)$ was developed by Tong (1983). Here the ECT is defined as

$$
\mathrm{ECT}_{\mathrm{t}-1}=\mathrm{I}_{\mathrm{t}} \mathrm{u}_{\mathrm{t}-1}+\left(1-\mathrm{I}_{\mathrm{t}}\right) \mathrm{u}_{\mathrm{t}-1}
$$

where $\mathrm{I}_{\mathrm{t}}$ represents a Heaviside indicator for different states of $\mathrm{u}_{\mathrm{t}-1}$ such that 


$$
I_{t}=\left\{\begin{array}{lll}
1 & \text { if } & u_{t-1} \geq 0 \\
0 & \text { if } & u_{t-1}<0
\end{array}\right.
$$

Using this definition we estimate

$$
\Delta u_{t}=I_{t} \rho_{1} u_{t-1}+\left(1-I_{t}\right) \rho_{2} u_{t-1}+\sum_{i=1}^{m^{*}} \rho_{2+i} \Delta u_{t-i}+\varepsilon_{t}
$$

Cointegration testing takes the form of a modified ADF test. The null of no cointegration is rejected if the estimated F-statistic for $\mathrm{H}_{0}: \rho_{1}=\rho_{2}=0$ is statistically significant based on critical values provided by Enders and Siklos (2000). If cointegration is established, an F-test for $H_{0}: \rho_{1}=\rho_{2}$ indicates the presence of asymmetry.

In the TAR* model the Heaviside indicator in conjunction with equation $(\mathrm{C} 8)^{20}$ is defined as

$$
I_{t}=\left\{\begin{array}{lll}
1 & \text { if } & u_{t-1} \geq a_{0}^{*} \\
0 & \text { if } & u_{t-1}<a_{0}^{*}
\end{array}\right.
$$

Following Chan (1993), the optimal threshold $\mathrm{a}_{0} *$ is found by searching over the mid- $80 \%$ of the distribution of $u_{t}$ and selecting the model for which the residual sum of squares is minimized. Cointegration and asymmetry testing proceeds with the above-described F-tests.

For the B-TAR* model, the Heaviside indicator in conjunction with equation $(\mathrm{C} 8)$ is now defined as

$$
I_{j t}=\left\{\begin{array}{ccccc}
I_{1 t}=1 \text { if } & u_{t-1} \geq a_{0}^{*} & \text { and } & 0 & \text { otherwise } \\
I_{2 t}=1 \text { if } & \left|u_{t-1}\right|<a_{0}^{*} & \text { and } & 0 & \text { otherwise } \\
I_{3 \mathrm{t}}=1 \text { if } & u_{t-1} \leq-a_{0}^{*} & \text { and } & 0 & \text { otherwise }
\end{array}\right.
$$

while equation $(\mathrm{C} 10)$ is modified to

$$
\Delta u_{t}=I_{1 t} \rho_{1} u_{t-1}+I_{2 t} \rho_{2} u_{t-1}+I_{3 t} \rho_{3} u_{t-1}+\sum_{i=1}^{m^{*}} \rho_{3+i} \Delta u_{t-i}+\varepsilon_{t}
$$

The F-tests for cointegration and asymmetry are now applied to all three coefficients $\rho_{\mathrm{j}}$.

\footnotetext{
${ }^{20}$ For both, the TAR* and the following B-TAR* model, the optimal lag length $\mathrm{m}^{*}$ of the $\mathrm{TAR}^{0}$ specification is used. Correspondingly, the optimal lag length of the M-TAR ${ }^{0}$ model is used for the M-TAR* model.
} 
In the $\mathrm{MTAR}^{0}$ and MTAR* models, the Heaviside indicator depends on the change in the error correction term $\Delta \mathrm{u}_{\mathrm{t}}$ such that

$$
I_{t}=\left\{\begin{array}{lll}
1 & \text { if } & \Delta \mathrm{u}_{\mathrm{t}-1} \geq \mathrm{a}_{0} \\
0 & \text { if } & \Delta \mathrm{u}_{\mathrm{t}-1}<\mathrm{a}_{0}
\end{array}\right.
$$

with $\mathrm{a}_{0}=0$ for the $\operatorname{MTAR}^{0}$ model and $\mathrm{a}_{0}=\mathrm{a}_{0}$ * for the MTAR* model. The optimal threshold is found using the same procedure as for the optimal threshold in the TAR* model. Equation (C10) remains unchanged as do the cointegration and asymmetry tests.

\section{$\underline{\text { Multipliers }}$}

The impact multiplier is given by the estimated coefficient $\beta_{1}$ in equation (1), (2), or (3). Regarding the long-run multiplier note that the long-run relationship between market rates and retail rates given by equation (4) can be interpreted either as a cointegration relationship or as the long-run solution of the VAR. Therefore, the long-term multiplier $\theta$ can be directly obtained from estimating equation (4) if the rates are cointegrated - that is if the pass-through model take the form of SYM, TAR ${ }^{0}, \mathrm{TAR}^{*}, \mathrm{BTAR}^{*}, \mathrm{MTAR}^{0}$, or MTAR*. Otherwise if the pass-through model takes the form STD or STD_LL, the long-term multiplier has to be calculated from (1) or (2) as:

$$
\theta=\frac{\beta_{1}+\sum_{\mathrm{i}=1}^{\mathrm{n}^{*}} \beta_{M, \mathrm{i}}}{1-\sum_{\mathrm{i}=1}^{\mathrm{k}^{*}} \beta_{B R, \mathrm{i}}}
$$

A full pass-through in the long run is reflected by $\theta=1$. An imperfect pass-through $(\theta<1)$ could be caused by a less than perfect elasticity of demand for banking products, the existence of market power, a lack of market contestability, switching costs, or information asymmetries. If the long-run pass-through is found to be overshooting $(\theta>1)$ in lending markets, this can be interpreted as a situation where banks increase lending rates to compensate for higher risks instead of rationing credit. 
In the second stage analysis, size is defined as the value of the long-run multiplier $(\theta)$. Speed is defined as the impact and intermediate multipliers. We use the software package RATS to estimate intermediate multipliers for $1,3,6$, and 12 months in response to shocks of $+1 \%$. $-1 \%,+0.25 \%$, and $-0.25 \%$. Here a peculiarity of the TAR-type models must be noted: As the speed of adjustment in a TAR-type pass-through model depends on the model's estimated error, multipliers cannot simply be obtained by comparing two sets of forecasts with and without changes in the explanatory variable $\mathrm{M}$ - at any point in time. As in all nonlinear models, multipliers obtained this way apply only to the particular context. In order to provide 'general' or 'state-independent' multipliers, we calculate them based on an equilibrium situation. In particular, we force the money market rate $M$ to zero at the end of each estimation period and allow the retail bank rate to converge to an equilibrium state before the shock in the money market rate is simulated. The multipliers are then calculated based on the resulting bank retail rate's adjustment. Note that in some cases the TAR-type pass-through models are not converging to an equilibrium state. In these cases we would only obtain state-dependent multipliers. To avoid this, we decided to select the next-best converging pass-through model (SYM or STD). For time deposits we decided to delete the non-converging sup-periods given the large number of rates available. Such problems are, however, rare and are indicated clearly in Table B6 of appendix B. 
Table 1: Macro-economic development, financial sector development and financial structure in CEECs

\begin{tabular}{|c|c|c|c|c|c|c|c|c|c|c|c|}
\hline & \multicolumn{3}{|c|}{ macro-economic development $^{1}$} & \multicolumn{5}{|c|}{ financial sector development } & \multicolumn{3}{|c|}{ financial structure } \\
\hline & $\begin{array}{r}\text { growth } \\
\text { (in \%) }\end{array}$ & $\begin{array}{r}\text { inflation } \\
\text { (in \%) }\end{array}$ & $\begin{array}{l}\text { money market } \\
\text { rate volatility }\end{array}$ & $\begin{array}{r}\text { EBRD index } \\
\text { of banking } \\
\text { sector reform }\end{array}$ & $\begin{array}{r}\text { private } \\
\text { credit to GDP } \\
\text { (in \%) }\end{array}$ & $\begin{array}{r}\text { stock market } \\
\text { capitalization } \\
\text { (in \% of GDP) }\end{array}$ & $\begin{array}{r}\text { net interest } \\
\text { margin } \\
(\text { in \%) }\end{array}$ & $\begin{array}{r}\text { performing } \\
\text { loans in } \% \text { of } \\
\text { total loans }\end{array}$ & $\begin{array}{r}\text { CR } 3 \text { asset } \\
\text { concentration } \\
(\text { in } \%)\end{array}$ & $\begin{array}{r}\text { foreign banks } \\
\text { in } \% \text { of all } \\
\text { banks }\end{array}$ & $\begin{array}{r}\text { state-owned } \\
\text { banks } \\
\text { (in \%) } \\
\end{array}$ \\
\hline \multicolumn{12}{|l|}{ Czech Republic } \\
\hline 1995 & 1,8 & 6,4 & 4,6 & 3,0 & 47 & 30 & 3,0 & 27 & 47 & 42 & 18 \\
\hline $2002^{2}$ & 2,4 & 2,3 & 1,7 & 3,7 & 20 & 21 & 3,1 & 9 & 48 & 70 & 5 \\
\hline \multicolumn{11}{|l|}{ Estonia } & 10 \\
\hline $2002^{2}$ & 4,8 & 3,5 & 2,1 & 3,7 & 30 & 34 & 4,0 & 1 & 80 & 57 & 0 \\
\hline \multicolumn{12}{|l|}{ Hungary } \\
\hline 1995 & 3,3 & 16,6 & 4,5 & 3,0 & 23 & 6 & 5,6 & $7^{3}$ & 44 & 49 & 49 \\
\hline $2002^{2}$ & 3,9 & 7,8 & 2,8 & 4,0 & 34 & 17 & 5,5 & 5 & 36 & 71 & 11 \\
\hline \multicolumn{11}{|l|}{ Latvia } & 10 \\
\hline $2002^{2}$ & 6,2 & 2,4 & 1,4 & 3,7 & 33 & 8 & 3,4 & 2 & 35 & 63 & 4 \\
\hline \multicolumn{12}{|l|}{ Lithuania } \\
\hline $2002^{2}$ & 4,9 & 0,4 & 2,0 & 3,0 & 14 & 10 & 3,9 & 6 & 67 & 29 & 0 \\
\hline \multicolumn{6}{|l|}{ Poland } & 4 & 7,5 & 24 & 43 & 22 & 72 \\
\hline $2002^{2}$ & 2,8 & 5,1 & 4,4 & 3,3 & 15 & 14 & 3,2 & 25 & 37 & 76 & 27 \\
\hline \multicolumn{12}{|l|}{ Slovak Republic } \\
\hline 1995 & 4,4 & 7,2 & 5,5 & 2,7 & 26 & 7 & 3,0 & 41 & 78 & 55 & 61 \\
\hline $2002^{2}$ & 3,2 & 8,4 & 3,1 & 3,3 & 25 & 7 & 2,8 & 11 & 61 & 83 & 3 \\
\hline Siovenia & 4,3 & 8,1 & 3,8 & 3,0 & 27 & 2 & 4,1 & 9 & 53 & 15 & 42 \\
\hline $2002^{2}$ & 3,6 & 7,4 & 1,1 & 3,3 & 41 & 19 & 3,3 & $10^{4}$ & 56 & 27 & 49 \\
\hline
\end{tabular}

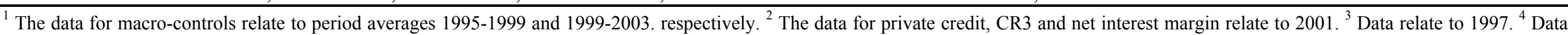

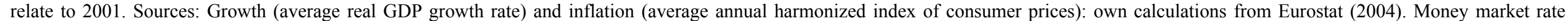

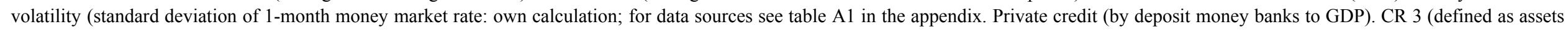

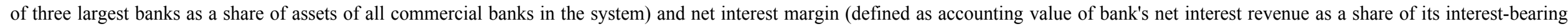

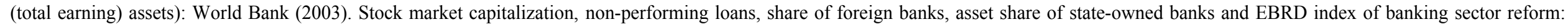
EBRD 2003 and own calculations. "na" indicates that data are not available. 
Table 2: Country, rate, and time patterns in the size and speed of the pass-througl

\begin{tabular}{|c|c|c|c|c|c|c|c|c|c|c|c|c|c|c|c|c|c|c|}
\hline & \multirow{2}{*}{$\begin{array}{l}\text { Impact } \\
\text { multiplier }\end{array}$} & \multicolumn{4}{|c|}{ muitipliers for + $1 \%$ shock } & \multicolumn{4}{|c|}{ muitipiers for - $1 \%$ shock } & \multicolumn{4}{|c|}{ multiphers for $+0.25 \%$ snock } & \multicolumn{4}{|c|}{ multiplers tor $-0.25 \%$ shock } & \multirow{2}{*}{$\begin{array}{r}\text { Iongrun } \\
\text { multiplier }\end{array}$} \\
\hline \multirow{2}{*}{\multicolumn{19}{|c|}{$\begin{array}{l}\text { Panel A: Regressions using a panel of all loan and deposit rate multiplier: } \\
\end{array}$}} \\
\hline & & & & & & & & & & & & & & & & & & \\
\hline \multirow[t]{2}{*}{ intercept } & 0,33 & 0,42 & 0,50 & 0,54 & 0,48 & 0,42 & 0,54 & 0,56 & 0,49 & 0,42 & 0,50 & 0,54 & 0,48 & 0,42 & 0,50 & 0,54 & 0,48 & 0,38 \\
\hline & 4,65 & 5,36 & 5,51 & 5,55 & 4,32 & 5,36 & 5,79 & 5,67 & 4,35 & 5,36 & 5,47 & 5,52 & 4,29 & 5,36 & 5,51 & 5,50 & 4,28 & 2,48 \\
\hline \multirow[t]{2}{*}{ Czech Republic } & $-0,01$ & $-0,17$ & $-0,34$ & $-0,40$ & $-0,35$ & $-0,17$ & $-0,32$ & $-0,38$ & $-0,33$ & $-0,17$ & $-0,33$ & $-0,39$ & $-0,34$ & $-0,17$ & $-0,33$ & $-0,39$ & $-0,34$ & $-0,26$ \\
\hline & $-0,15$ & $-3,74$ & $-6,43$ & $-7,07$ & $-5,39$ & $-3,74$ & $-6,03$ & $-6,69$ & $-5,16$ & $-3,74$ & $-6,35$ & $-7,00$ & $-5,36$ & $-3,74$ & $-6,37$ & $-6,99$ & $-5,36$ & $-2,96$ \\
\hline Estonia & 2,29 & $-1,08$ & $-4,64$ & $-5,92$ & $-5,39$ & $-1,08$ & $-4,57$ & $-5,77$ & $-5,27$ & $-1,08$ & $-4,56$ & $-5,83$ & $-5,31$ & $-1,08$ & $-4,58$ & $-5,82$ & $-5,30$ & $-3,57$ \\
\hline \multirow[t]{2}{*}{ Hungary } & 0,15 & 0,19 & 0,13 & 0,08 & 0,09 & 0,19 & 0,14 & 0,11 & 0,10 & 0,19 & 0,13 & 0,08 & 0,09 & 0,19 & 0,12 & 0,09 & 0,09 & 0,10 \\
\hline & 3,68 & 4,16 & 2,41 & 1,48 & 1,34 & 4,16 & 2,66 & 1,88 & 1,60 & 4,16 & 2,37 & 1,49 & 1,37 & 4,16 & 2,30 & 1,58 & 1,44 & 1,15 \\
\hline \multirow{2}{*}{ Latvia } & 0,06 & $-0,07$ & $-0,27$ & $-0,35$ & $-0,32$ & $-0,07$ & $-0,27$ & $-0,34$ & $-0,32$ & $-0,07$ & $-0,27$ & $-0,34$ & $-0,32$ & $-0,07$ & $-0,27$ & $-0,34$ & $-0,32$ & $-0,37$ \\
\hline & 1,24 & $-1,22$ & $-4,39$ & $-5,18$ & $-4,23$ & $-1,22$ & $-4,22$ & $-4,97$ & $-4,11$ & $-1,22$ & $-4,36$ & $-5,13$ & $-4,19$ & $-1,22$ & $-4,36$ & $-5,10$ & $-4,18$ & $-3,52$ \\
\hline Lithuania & $-0,10$ & $-0,19$ & $-0,38$ & $-0,43$ & $-0,36$ & $-0,19$ & $-0,37$ & $-0,43$ & $-0,35$ & $-0,19$ & $-0,38$ & $-0,43$ & $-0,35$ & $-0,19$ & $-0,37$ & $-0,43$ & $-0,35$ & $-0,22$ \\
\hline \multirow[t]{2}{*}{ Slovak Republic } & $-0,06$ & $-0,23$ & $-0,49$ & $-0,60$ & $-0,60$ & $-0,23$ & $-0,48$ & $-0,60$ & $-0,59$ & $-0,23$ & $-0,48$ & $-0,60$ & $-0,59$ & $-0,23$ & $-0,48$ & $-0,60$ & $-0,60$ & $-0,57$ \\
\hline & $-1,57$ & $-5,43$ & $-9,95$ & $-11,42$ & $-9,97$ & $-5,43$ & $-9,67$ & $-11,10$ & $-9,79$ & $-5,43$ & $-9,86$ & $-11,33$ & $-9,89$ & $-5,43$ & $-9,89$ & $-11,30$ & $-9,89$ & $-6,79$ \\
\hline \multirow[t]{2}{*}{ Slovenia } & 0,27 & 0,27 & 0,23 & 0,27 & 0,42 & 0,27 & 0,23 & 0,28 & 0,43 & 0,27 & 0,23 & 0,28 & 0,43 & 0,27 & 0,23 & 0,28 & 0,43 & 0,62 \\
\hline & 7,33 & 6,79 & 4,81 & 5,38 & 7,29 & 6,79 & 4,78 & 5,35 & 7,30 & 6,79 & 4,93 & 5,49 & 7,38 & 6,79 & 4,93 & 5,44 & 7,35 & 7,77 \\
\hline \multirow{2}{*}{ consumer loans } & 0,01 & $-0,05$ & $-0,03$ & $-0,04$ & $-0,08$ & $-0,05$ & $-0,07$ & $-0,07$ & $-0,10$ & $-0,05$ & $-0,02$ & $-0,04$ & $-0,08$ & $-0,05$ & $-0,02$ & $-0,04$ & $-0,08$ & $-0,16$ \\
\hline & 0,21 & $-0,84$ & $-0,39$ & $-0,51$ & $-1,03$ & $-0,84$ & $-1,01$ & $-0,96$ & $-1,25$ & $-0,84$ & $-0,32$ & $-0,50$ & $-1,01$ & $-0,84$ & $-0,34$ & $-0,49$ & $-1,00$ & $-1,36$ \\
\hline \multirow{2}{*}{ short-term corporate loans } & 0,25 & 0,20 & 0,24 & 0,22 & 0,18 & 0,20 & 0,20 & 0,19 & 0,17 & 0,20 & 0,24 & 0,23 & 0,18 & 0,20 & 0,24 & 0,23 & 0,19 & 0,09 \\
\hline & 4,55 & 3,43 & 3,47 & 3,00 & 2,16 & 3,43 & 2,80 & 2,54 & 1,95 & 3,43 & 3,47 & 3,03 & 2,17 & 3,43 & 3,46 & 3,02 & 2,17 & 0,77 \\
\hline \multirow[t]{2}{*}{ long-term corporate loans } & 0,21 & 0,19 & 0,24 & 0,22 & 0,19 & 0,19 & 0,19 & 0,20 & 0,17 & 0,19 & 0,23 & 0,22 & 0,18 & 0,19 & 0,23 & 0,23 & 0,19 & 0,10 \\
\hline & 3,81 & 3,04 & 3,35 & 2,91 & 2,15 & 3,04 & 2,58 & 2,55 & 1,96 & 3,04 & 3,22 & 2,86 & 2,11 & 3,04 & 3,28 & 3,04 & 2,20 & 0,86 \\
\hline current account deposits & 0,00 & $-0,20$ & $-0,36$ & $-0,48$ & $-0,53$ & $-0,20$ & $-0,41$ & $-0,52$ & $-0,55$ & $-0,20$ & $-0,35$ & $-0,48$ & $-0,52$ & $-0,20$ & $-0,35$ & $-0,48$ & $-0,52$ & $-0,51$ \\
\hline & $-0,02$ & $-2,55$ & $-3,97$ & $-5,00$ & $-4,79$ & $-2,55$ & $-4,44$ & $-5,29$ & $-4,93$ & $-2,55$ & $-3,91$ & $-4,94$ & $-4,75$ & $-2,55$ & $-3,92$ & $-4,94$ & $-4,76$ & $-3,32$ \\
\hline time deposits & 0,10 & 0,05 & 0,08 & 0,04 & $-0,0$ & 0,05 & 0,04 & 0,01 & -0, & 0,05 & 0,08 & 0,04 & -0, & 0,05 & 0,08 & 0,04 & $-0,02$ & $-0,09$ \\
\hline & 2,31 & 0,99 & 1,40 & 0,61 & $-0,30$ & 0,99 & 0,60 & 0,10 & $-0,55$ & 0,99 & 1,37 & 0,58 & $-0,32$ & 0,99 & 1,34 & 0,59 & $-0,30$ & $-0,93$ \\
\hline 1994-1998 & $-0,04$ & 0,10 & 0,15 & 0,16 & 0,17 & 0,10 & 0,15 & 0,16 & 0,1 & 0,10 & 0,14 & 0,16 & 0,17 & 0,10 & 0,14 & 0,16 & 0,17 & \\
\hline & $-0,60$ & 1,55 & 1,95 & 2,02 & 1,92 & 1,55 & 1,92 & 2,02 & 1,92 & 1,55 & 1,94 & 2,02 & 1,91 & 1,55 & 1,94 & 2,01 & 1,91 & 1,46 \\
\hline 1995-1999 & $-0,25$ & $-0,09$ & 0,07 & 0,17 & 0,24 & $-0,09$ & 0,07 & 0,18 & 0,25 & $-0,09$ & 0,07 & 0,17 & 0,24 & $-0,09$ & 0,07 & 0,17 & 0,24 & 0,31 \\
\hline & $-4,60$ & $-1,45$ & 1,03 & 2,32 & 2,88 & $-1,45$ & 0,96 & 2,33 & 2,8 & $-1,45$ & 0,96 & 2,28 & 2,85 & $-1,45$ & 0,96 & 2,32 & 2,88 & 2,60 \\
\hline 1996-2000 & $-0,30$ & $-0,15$ & 0,01 & 0,11 & 0,20 & $-0,15$ & 0,01 & 0,12 & 0,20 & $-0,15$ & 0,01 & 0,11 & 0,20 & $-0,15$ & 0,01 & 0,11 & 0,20 & 0,28 \\
\hline & $-5,47$ & $-2,59$ & 0,12 & 1,52 & 2,38 & $-2,59$ & 0,20 & 1,53 & 2,39 & $-2,59$ & 0,13 & 1,52 & 2,37 & $-2,59$ & 0,16 & 1,53 & 2,37 & 2,41 \\
\hline 1997-2001 & $-0,30$ & $-0,14$ & 0,06 & 0,19 & 0,34 & $-0,14$ & 0,06 & 0,20 & 0,35 & $-0,14$ & 0,06 & 0,19 & 0,34 & $-0,14$ & 0,06 & 0,19 & 0,34 & 0,45 \\
\hline & $-5,75$ & $-2,46$ & 0,84 & 2,69 & 4,16 & $-2,46$ & 0,95 & 2,74 & 4,19 & $-2,46$ & 0,87 & 2,71 & 4,17 & $-2,46$ & 0,87 & 2,68 & 4,15 & 3,95 \\
\hline 1998-2002 & $-0,32$ & $-0,16$ & 0,01 & 0,13 & 0,27 & $-0,16$ & 0,02 & 0,14 & 0,27 & $-0,16$ & 0,01 & 0,12 & 0,26 & $-0,16$ & 0,01 & 0,12 & 0,26 & 0,42 \\
\hline & $-6,15$ & $-2,77$ & 0,11 & 1,79 & 3,26 & $-2,77$ & 0,26 & 1,87 & 3,2 & $-2,77$ & 0,09 & 1,74 & 3,20 & $-2,77$ & 0,08 & 1,71 & 3,18 & 3,66 \\
\hline 1999-2003 & $-0,28$ & $-0,16$ & 0,02 & 0,16 & 0,32 & $-0,16$ & 0,02 & 0,16 & 0,32 & $-0,16$ & 0,02 & 0,16 & 0,32 & $-0,16$ & 0,02 & 0,16 & 0,32 & 0,62 \\
\hline & $-5,40$ & $-2,80$ & 0,27 & 2,21 & 3,98 & $-2,80$ & 0,31 & 2,19 & 3,97 & $-2,80$ & 0,32 & 2,24 & 3,99 & $-2,80$ & 0,31 & 2,21 & 3,97 & 5,56 \\
\hline adjusted $R^{2}$ & $50 \%$ & $57 \%$ & $62 \%$ & $66 \%$ & $64 \%$ & $57 \%$ & $61 \%$ & $65 \%$ & $64 \%$ & $57 \%$ & $62 \%$ & $66 \%$ & $64 \%$ & $57 \%$ & $62 \%$ & $66 \%$ & $64 \%$ & 54 \\
\hline
\end{tabular}


Table 2: Country, rate, and time patterns in the size and speed of the pass-througl

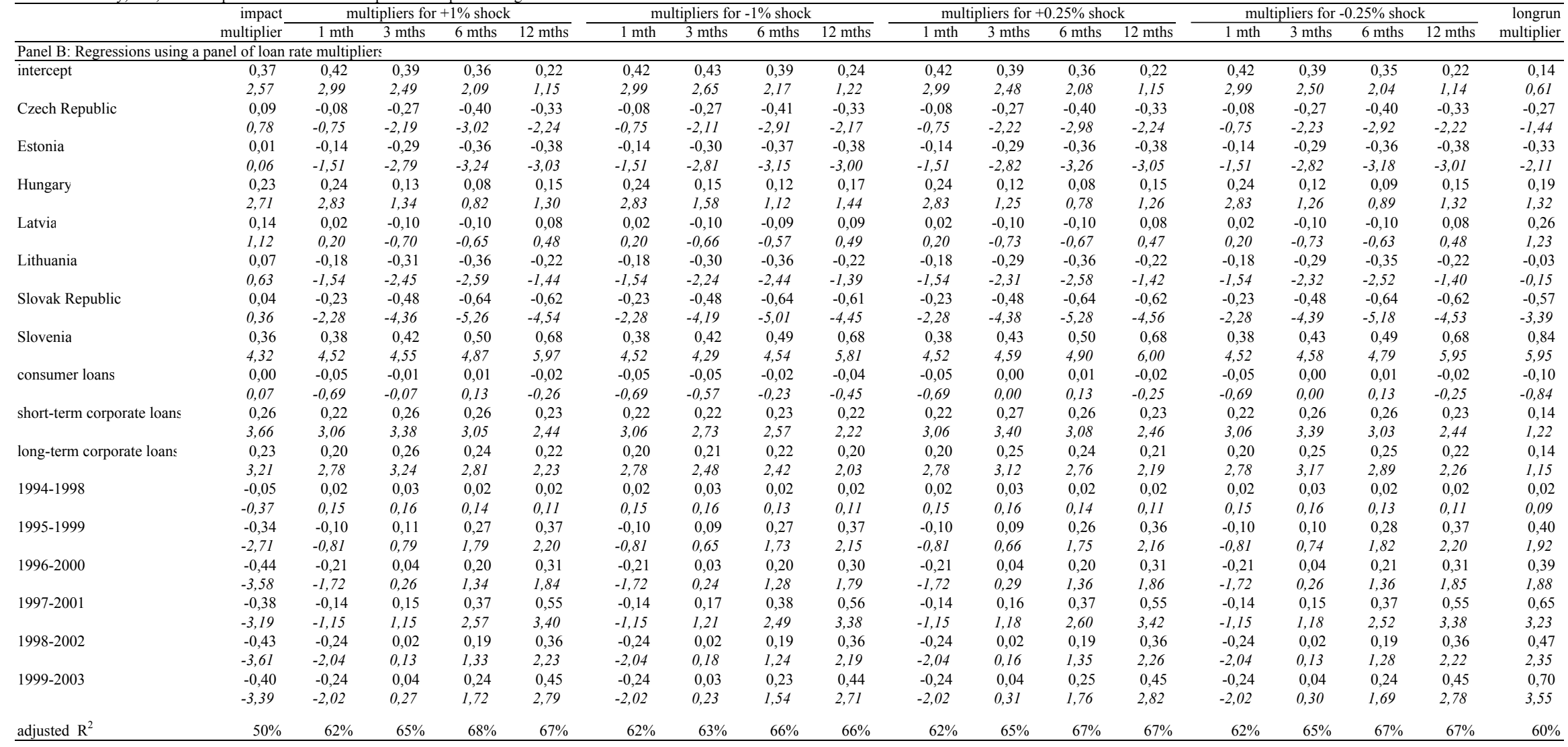


Table 2: Country, rate, and time patterns in the size and speed of the pass-througl

multipliers for $+1 \%$ shock multipliers for $-1 \%$ shock

multipliers for $+0.25 \%$ shock

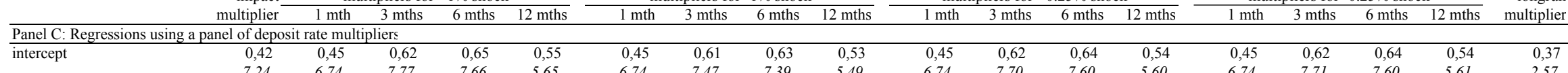

\begin{tabular}{|c|c|c|c|c|c|c|c|c|c|c|c|c|c|c|c|c|c|c|}
\hline intercept & 0,42 & 0,45 & 0,62 & 0,65 & 0,55 & 0,45 & 0,61 & 0,63 & 0,53 & 0,45 & 0,62 & 0,64 & 0,54 & 0,45 & 0,62 & 0,64 & 0,54 & 0,37 \\
\hline \multirow{3}{*}{ Czech Republic } & 7,24 & 6,74 & 7,77 & 7,66 & 5,65 & 6,74 & 7,47 & 7,39 & 5,49 & 6,74 & 7,70 & 7,60 & 5,60 & 6,74 & 7,71 & 7,60 & 5,61 & 2,57 \\
\hline & $-0,03$ & $-0,19$ & $-0,38$ & $-0,43$ & $-0,38$ & $-0,19$ & $-0,36$ & $-0,40$ & $-0,36$ & $-0,19$ & $-0,37$ & $-0,42$ & $-0,38$ & $-0,19$ & $-0,37$ & $-0,42$ & $-0,38$ & $-0,29$ \\
\hline & $-0,85$ & $-4,00$ & $-6,70$ & $-7,19$ & $-5,59$ & $-4,00$ & $-6,23$ & $-6,75$ & $-5,33$ & $-4,00$ & $-6,61$ & $-7,12$ & $-5,54$ & $-4,00$ & $-6,61$ & $-7,13$ & $-5,55$ & $-2,85$ \\
\hline \multirow[t]{2}{*}{ Estonia } & 0,17 & 0,02 & $-0,22$ & $-0,32$ & $-0,32$ & 0,02 & $-0,21$ & $-0,31$ & $-0,31$ & 0,02 & $-0,21$ & $-0,32$ & $-0,31$ & 0,02 & $-0,21$ & $-0,32$ & $-0,31$ & $-0,30$ \\
\hline & 3,77 & 0,38 & $-3,53$ & $-4,96$ & $-4,30$ & 0,38 & $-3,37$ & $-4,75$ & $-4,13$ & 0,38 & $-3,43$ & $-4,83$ & $-4,18$ & 0,38 & $-3,44$ & $-4,83$ & $-4,19$ & $-2,71$ \\
\hline \multirow[t]{2}{*}{ Hungary } & 0,12 & 0,16 & 0,20 & 0,15 & 0,11 & 0,16 & 0,19 & 0,15 & 0,12 & 0,16 & 0,20 & 0,16 & 0,11 & 0,16 & 0,19 & 0,15 & 0,11 & 0,10 \\
\hline & 2,49 & 2,74 & 2,95 & 2,14 & 1,29 & 2,74 & 2,81 & 2,14 & 1,40 & 2,74 & 3,01 & 2,18 & 1,36 & 2,74 & 2,85 & 2,13 & 1,38 & 0,83 \\
\hline \multirow[t]{2}{*}{ Latvia } & 0,04 & $-0,10$ & $-0,34$ & $-0,44$ & $-0,45$ & $-0,10$ & $-0,33$ & $-0,43$ & $-0,44$ & $-0,10$ & $-0,34$ & $-0,43$ & $-0,45$ & $-0,10$ & $-0,34$ & $-0,43$ & $-0,45$ & $-0,57$ \\
\hline & 0,77 & $-1,77$ & $-5,07$ & $-6,14$ & $-5,56$ & $-1,77$ & $-4,87$ & $-5,96$ & $-5,43$ & $-1,77$ & $-5,00$ & $-6,06$ & $-5,49$ & $-1,77$ & $-4,99$ & $-6,06$ & $-5,50$ & $-4,66$ \\
\hline \multirow[t]{2}{*}{ Lithuania } & $-0,13$ & $-0,19$ & $-0,42$ & $-0,47$ & $-0,41$ & $-0,19$ & $-0,41$ & $-0,46$ & $-0,40$ & $-0,19$ & $-0,41$ & $-0,47$ & $-0,41$ & $-0,19$ & $-0,41$ & $-0,47$ & $-0,41$ & $-0,28$ \\
\hline & $-3,66$ & $-4,67$ & $-8,49$ & $-9,08$ & $-6,93$ & $-4,67$ & $-8,14$ & $-8,84$ & $-6,76$ & $-4,67$ & $-8,37$ & $-8,95$ & $-6,82$ & $-4,67$ & $-8,33$ & $-8,95$ & $-6,83$ & $-3,10$ \\
\hline \multirow[t]{2}{*}{ Slovak Republic } & $\begin{array}{l}-0,09 \\
-\end{array}$ & $-0,23$ & $-0,51$ & $-0,62$ & $-0,62$ & $-0,23$ & $-0,50$ & $-0,60$ & $-0,61$ & $-0,23$ & $-0,50$ & $-0,61$ & $\begin{array}{l}-0,02 \\
-0,61\end{array}$ & $-0,23$ & $-0,50$ & $-0,61$ & $-0,61$ & $\begin{array}{l}-0,59 \\
-0,10\end{array}$ \\
\hline & $-2,47$ & $-5,19$ & $-9,61$ & $-11,00$ & $-9,61$ & $-5,19$ & $-9,31$ & $-10,72$ & $-9,44$ & $-5,19$ & $-9,50$ & $-10,87$ & $-9,50$ & $-5,19$ & $-9,51$ & $-10,87$ & $-9,52$ & $-6,13$ \\
\hline \multirow[t]{2}{*}{ Slovenia } & 0,22 & 0,22 & 0,11 & 0,15 & 0,29 & 0,22 & 0,12 & 0,16 & 0,30 & 0,22 & 0,12 & 0,16 & 0,30 & 0,22 & 0,12 & 0,16 & 0,30 & 0,53 \\
\hline & 5,68 & 4,77 & 2,09 & 2,64 & 4,49 & 4,77 & 2,25 & 2,84 & 4,62 & 4,77 & 2,19 & 2,76 & 4,59 & 4,77 & 2,21 & 2,77 & 4,59 & 5,44 \\
\hline \multirow[t]{2}{*}{ current account deposits } & $-0,12$ & $-0,24$ & $-0,50$ & $-0,58$ & $-0,55$ & $-0,24$ & $-0,49$ & $-0,57$ & $-0,55$ & $-0,24$ & $-0,50$ & $-0,57$ & $-0,55$ & $-0,24$ & $-0,49$ & $-0,57$ & $-0,55$ & $-0,46$ \\
\hline & $-2,20$ & $-3,94$ & $-6,81$ & $-7,51$ & $-6,22$ & $-3,94$ & $-6,58$ & $-7,33$ & $-6,15$ & $-3,94$ & $-6,74$ & $-7,40$ & $-6,16$ & $-3,94$ & $-6,67$ & $-7,39$ & $-6,17$ & $-3,45$ \\
\hline \multirow[t]{2}{*}{ savings accounts } & $-0,18$ & $-0,22$ & $-0,31$ & $-0,38$ & $-0,43$ & $-0,22$ & $-0,32$ & $-0,38$ & $-0,43$ & $-0,22$ & $-0,31$ & $-0,38$ & $-0,43$ & $-0,22$ & $-0,31$ & $-0,38$ & $-0,43$ & $-0,50$ \\
\hline & $-4,94$ & $-5,17$ & $-6,07$ & $-6,93$ & $-6,88$ & $-5,17$ & $-6,07$ & $-6,95$ & $-6,89$ & $-5,17$ & $-6,07$ & $-6,91$ & $-6,86$ & $-5,17$ & $-6,08$ & $-6,91$ & $-6,86$ & $-5,37$ \\
\hline \multirow[t]{2}{*}{ 1994-1998 } & $-0,01$ & 0,13 & 0,18 & 0,19 & 0,20 & 0,13 & 0,18 & 0,19 & 0,21 & 0,13 & 0,18 & 0,19 & 0,20 & 0,13 & 0,18 & 0,19 & 0,20 & 0,22 \\
\hline & $-0,17$ & 1,86 & 2,14 & 2,18 & 2,05 & 1,86 & 2,13 & 2,21 & 2,07 & 1,86 & 2,14 & 2,17 & 2,04 & 1,86 & 2,13 & 2,17 & 2,04 & 1,50 \\
\hline \multirow[t]{2}{*}{ 1995-1999 } & $-0,21$ & $-0,08$ & 0,06 & 0,14 & 0,20 & $-0,08$ & 0,06 & 0,14 & 0,20 & $-0,08$ & 0,06 & 0,13 & 0,20 & $-0,08$ & 0,06 & 0,13 & 0,20 & 0,27 \\
\hline & $-3,76$ & $-1,19$ & 0,76 & 1,66 & 2,08 & $-1,19$ & 0,76 & 1,70 & 2,13 & $-1,19$ & 0,76 & 1,64 & 2,07 & $-1,19$ & 0,71 & 1,63 & 2,08 & 1,95 \\
\hline \multirow[t]{2}{*}{ 1996-2000 } & $-0,24$ & $-0,13$ & $-0,01$ & 0,08 & 0,15 & $-0,13$ & 0,00 & 0,08 & 0,16 & $-0,13$ & $-0,01$ & 0,07 & 0,15 & $-0,13$ & 0,00 & 0,07 & 0,15 & 0,24 \\
\hline & $-4,38$ & $-2,08$ & $-0,07$ & 0,93 & 1,64 & $-2,08$ & 0,03 & 0,96 & 1,68 & $-2,08$ & $-0,08$ & 0,91 & 1,63 & $-2,08$ & $-0,03$ & 0,91 & 1,63 & 1,71 \\
\hline \multirow[t]{2}{*}{ 1997-2001 } & $-0,26$ & $-0,14$ & 0,02 & 0,13 & 0,26 & $-0,14$ & 0,03 & 0,14 & 0,27 & $-0,14$ & 0,02 & 0,13 & 0,26 & $-0,14$ & 0,02 & 0,13 & 0,26 & 0,38 \\
\hline & $-4,86$ & $-2,14$ & 0,31 & 1,60 & 2,89 & $-2,14$ & 0,40 & 1,69 & 2,94 & $-2,14$ & 0,32 & 1,60 & 2,88 & $-2,14$ & 0,32 & 1,60 & 2,88 & 2,78 \\
\hline \multirow[t]{2}{*}{ 1998-2002 } & $-0,28$ & $-0,12$ & 0,01 & 0,11 & 0,23 & $-0,12$ & 0,02 & 0,12 & 0,24 & $-0,12$ & 0,00 & 0,10 & 0,23 & $-0,12$ & 0,00 & 0,10 & 0,23 & 0,40 \\
\hline & $-5,08$ & $-1,91$ & 0,10 & 1,36 & 2,57 & $-1,91$ & 0,26 & 1,51 & 2,64 & $-1,91$ & 0,06 & 1,27 & 2,49 & $-1,91$ & 0,06 & 1,27 & 2,48 & 2,95 \\
\hline \multirow[t]{2}{*}{ 1999-2003 } & $-0,24$ & $\begin{array}{l}-0,12 \\
-0,12\end{array}$ & 0,02 & 0,13 & 0,28 & $\begin{array}{l}-1,12 \\
-0,12\end{array}$ & 0,02 & 0,14 & 0,28 & $-0,12$ & 0,02 & 0,13 & 0,28 & $-0,12$ & 0,02 & 0,13 & 0,28 & 0,60 \\
\hline & $-4,40$ & $-1,86$ & 0,22 & 1,63 & 3,06 & $-1,86$ & 0,30 & 1,72 & 3,11 & $-1,86$ & 0,23 & 1,63 & 3,06 & $-1,86$ & 0,23 & 1,63 & 3,06 & 4,43 \\
\hline adjusted $R^{2}$ & $51 \%$ & $51 \%$ & $58 \%$ & $63 \%$ & $61 \%$ & $51 \%$ & $57 \%$ & $62 \%$ & $60 \%$ & $51 \%$ & $58 \%$ & $63 \%$ & $60 \%$ & $51 \%$ & $58 \%$ & $63 \%$ & $60 \%$ & $48 \%$ \\
\hline
\end{tabular}

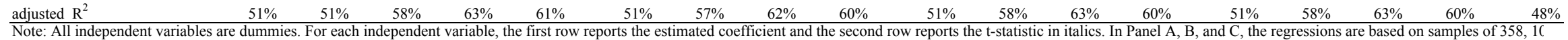
and 256 observations respectively. 


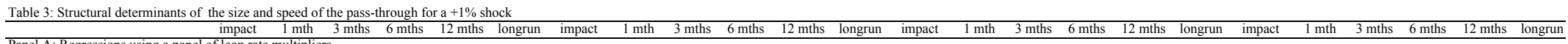

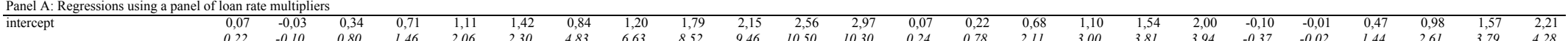

inflation

bankreform

CR3

badloans

frobankp

frgbankp * (1 - Slovenia dummy)

Slovenia dummy

short-term corporate loan dummy

long-term corporate loan dummy

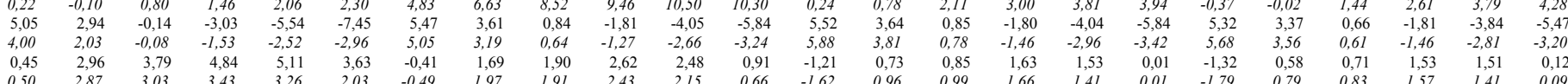

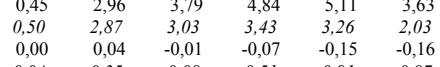

adjusted $R^{2}$

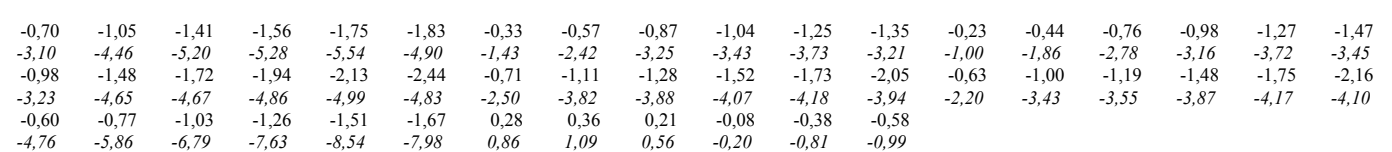

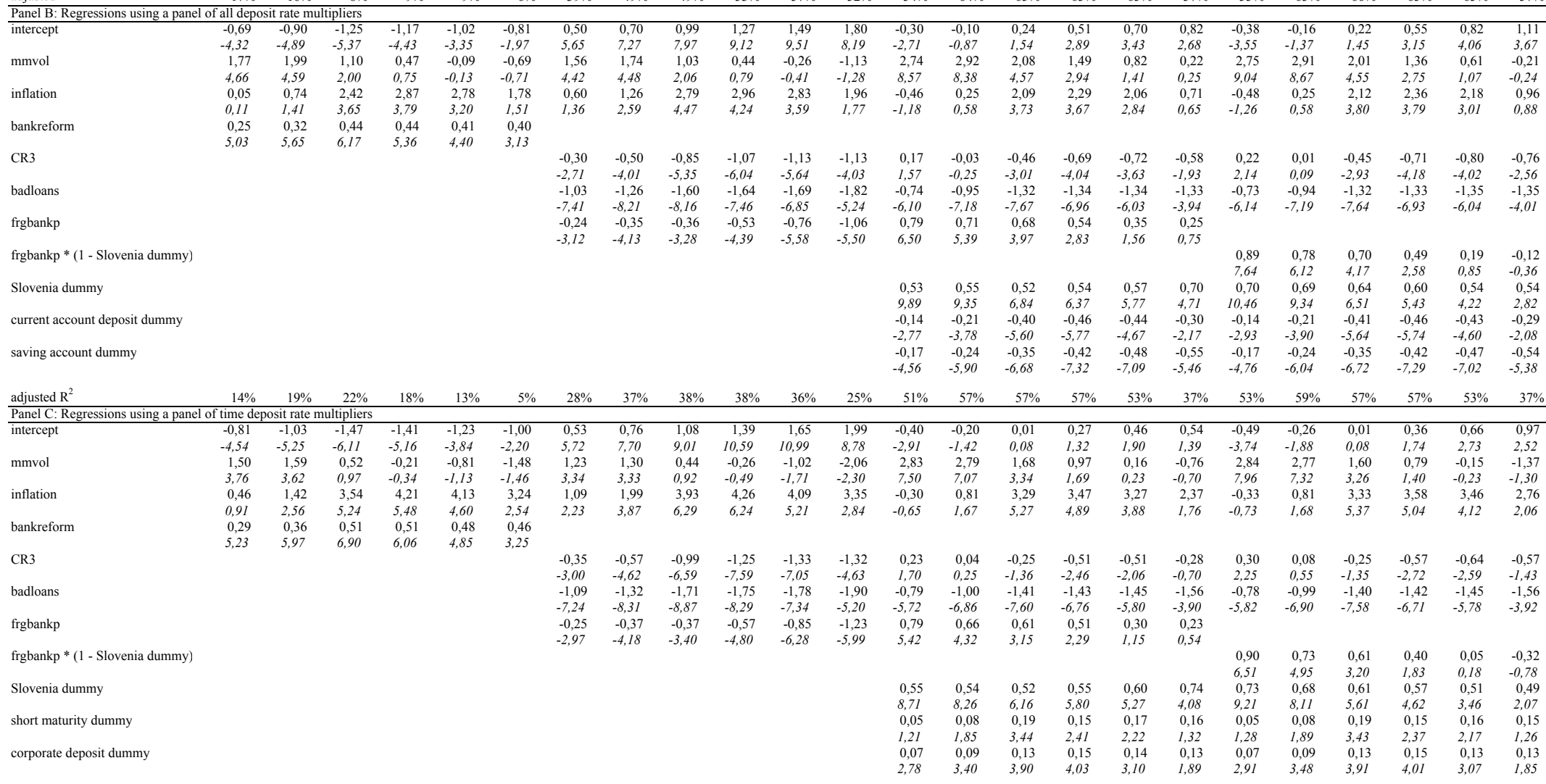

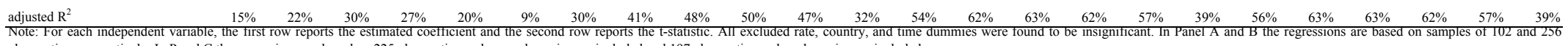
observations respectively. In Panel C the regressions are based on 225 observations when no dummies are included and 197 observations when dummies are included. 


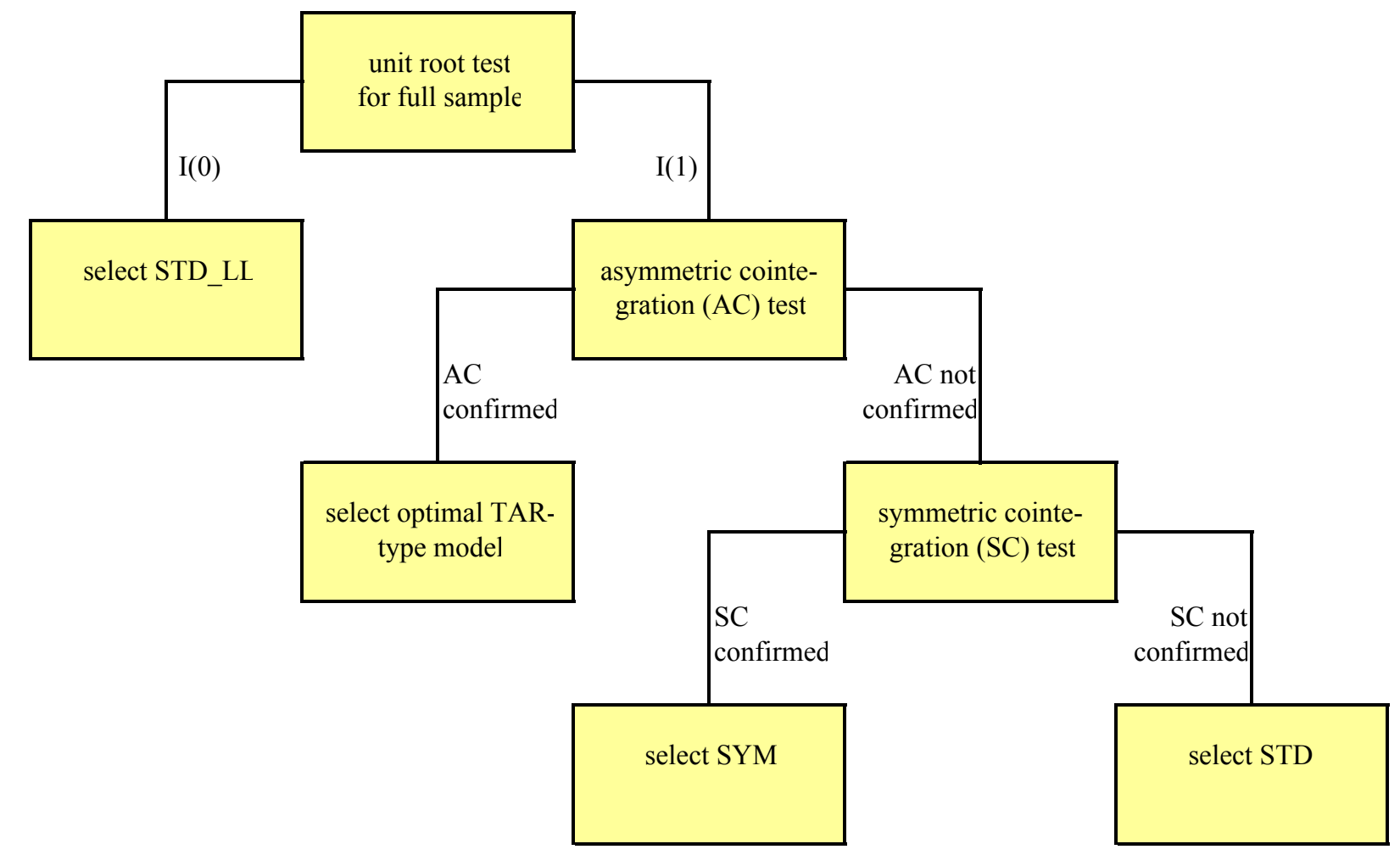




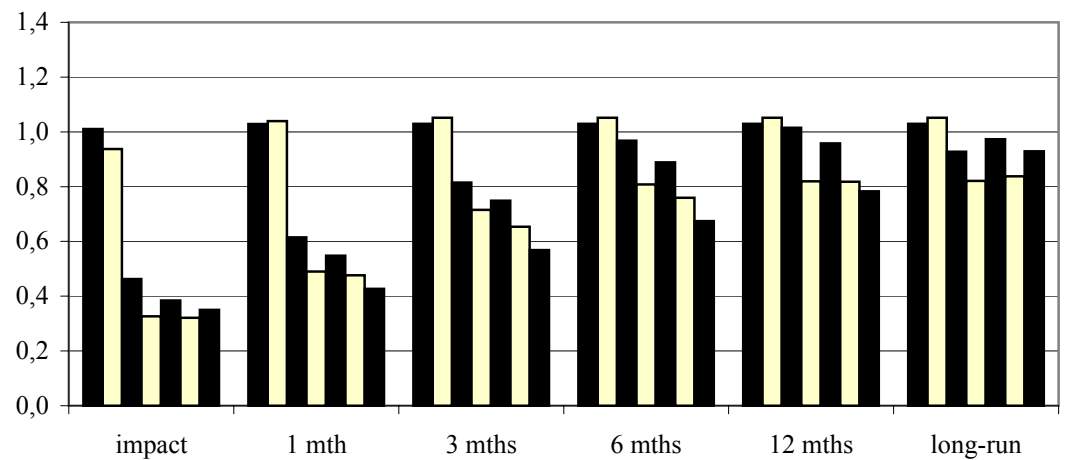

Panel B: Long-term corporate loan rate multipliers

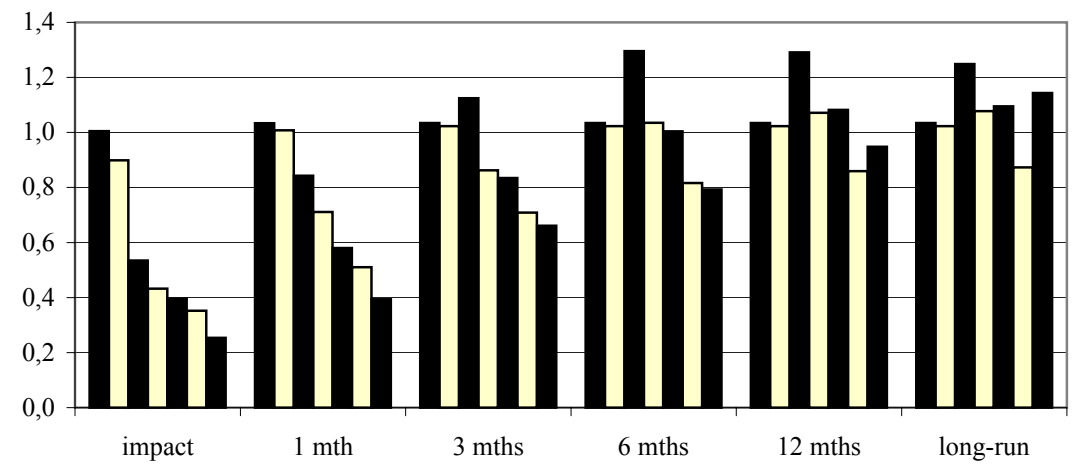

Panel C: Consumer loan rate multipliers

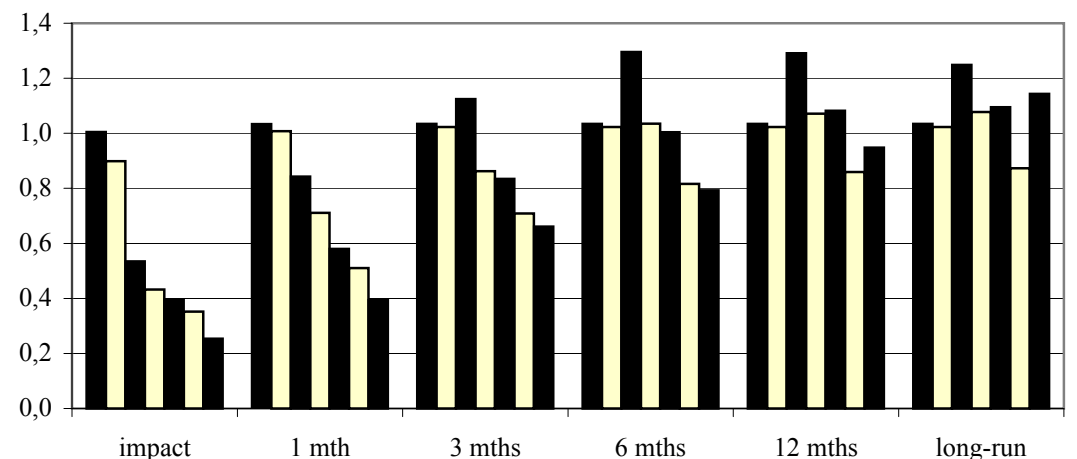

Panel D: Mortgage rate multipliers

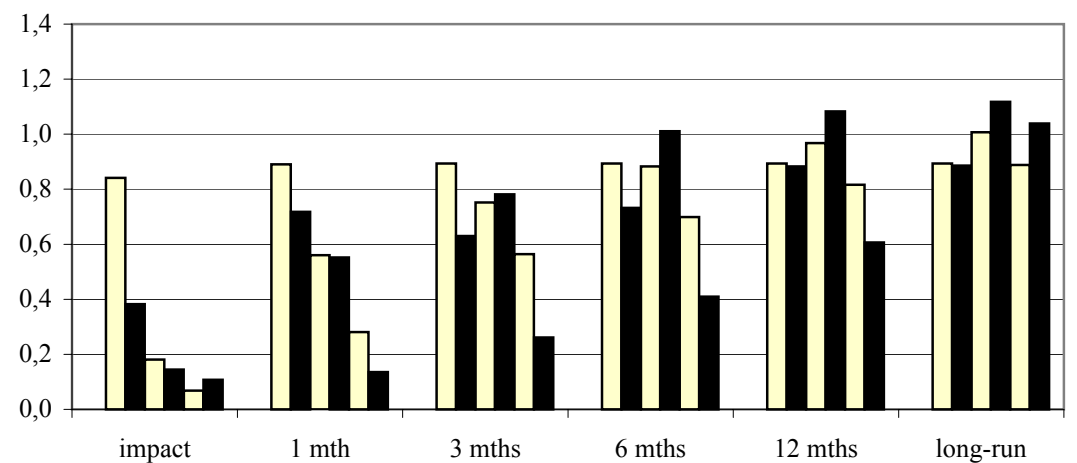


Figure 2: The average pass-through

Panel E: Current account deposit rate multipliers

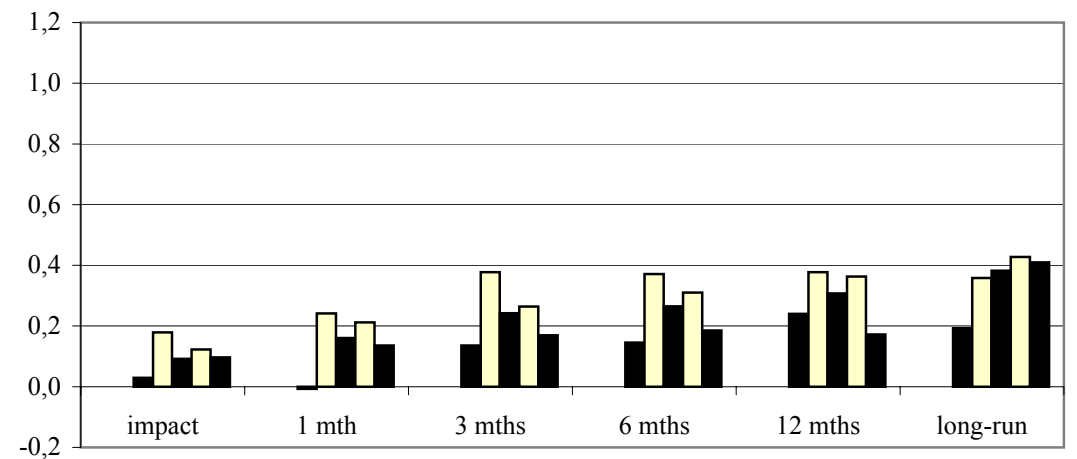

Panel F: Saving account rate multipliers

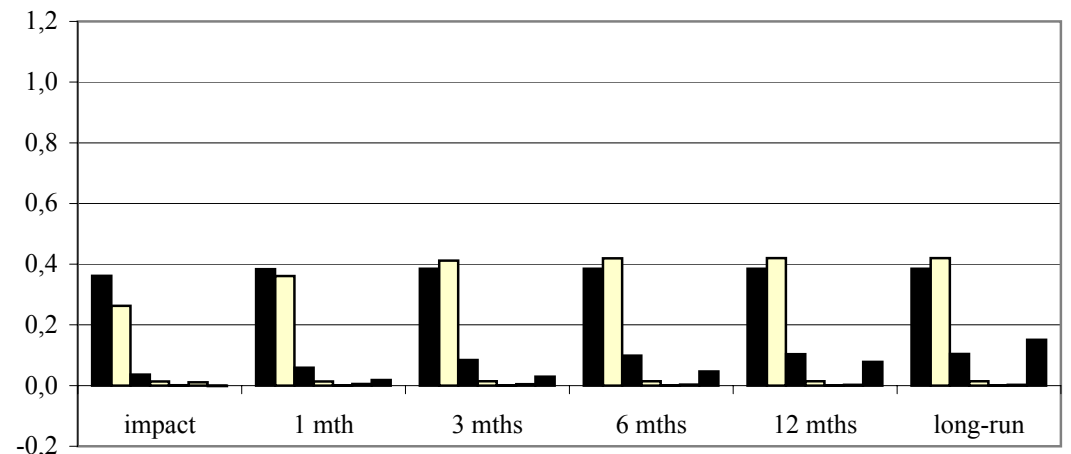

\section{Panel G: Time deposit rate multipliers}

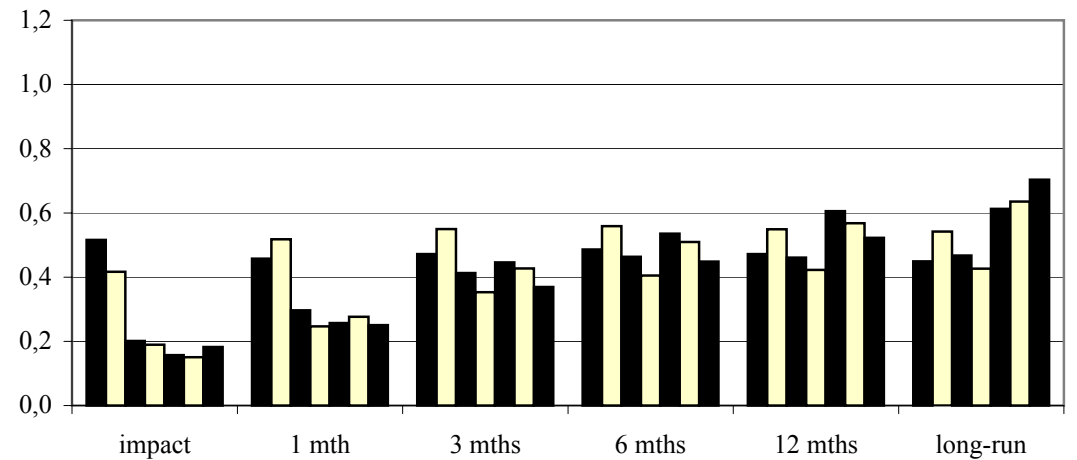

For each category in the panels, the bars represent different multipliers as following from left to right: 1993-1997, 1994-1998, 1995-1999, 1996-2000, 1997-2001, 1998-2002, and 1999-2003. Exceptions are Panel D and E which start on the left with 1994-1998 and 1995-1999, respectively. 


\begin{tabular}{|c|c|c|c|c|}
\hline \multirow{2}{*}{ country } & \multirow[b]{2}{*}{ source } & \multirow[b]{2}{*}{ description } & \multicolumn{2}{|c|}{ data availability } \\
\hline & & & to & from \\
\hline \multicolumn{5}{|c|}{ Panel A: Monetary policy rates } \\
\hline Czech Republic & $\mathrm{CB}$ & $\begin{array}{l}\text { Money and banking statistics } \gg>>\text { National money and banking statistics }>>>\text { Interest rates } \\
\text { > > PRIBOR: Average month PRIBOR rates: } 1 \text { month in } \%\end{array}$ & 1993,01 & 2003,12 \\
\hline Estonia & $\mathrm{CB}$ & 6.3.4.2 TALIBOR monthly arithmetic means: 1 month & 1996,01 & 2003,12 \\
\hline Hungary & $\begin{array}{c}\text { IFS \& } \\
\text { CB }\end{array}$ & $\begin{array}{l}\text { IFS line } 60 \text { discount rate until 12/99. CB's 1-month unsecured HUF interbank lending } \\
\text { transactions starting } 1 / 00 \text {. }\end{array}$ & 1990,01 & 2003,12 \\
\hline Latvia & $\begin{array}{c}\text { IFS \& } \\
\text { CB }\end{array}$ & $\begin{array}{l}\text { For all data CB's table 19a: Interest rates in the interbank market (\% p.a.) -- Interest rates on } \\
\text { loans in lats - up to } 1 \text { month. Exception: IFS line 60b for Aug-Dec } 1993 \text { and Oct-Dec } 2003\end{array}$ & 1993,08 & 2003,12 \\
\hline Lithuania & IFS & Money market rate line $60 \mathrm{~b}$ & 1993,12 & 2003,12 \\
\hline Poland & IFS & Money market rate line $60 \mathrm{~b}$ & 1990,12 & 2003,06 \\
\hline Slovak Republic ${ }^{1}$ & $\mathrm{CB}$ & Bratislava interbank offered rate BRIBOR: offer rate, monthly average - 1 month maturity & 1995,07 & 2003,12 \\
\hline Slovenia $^{2}$ & $\mathrm{CB}$ & $\begin{array}{l}\text { Monthly Bulletin of the Bank of Slovenia Section 1. Financial Markets, Table 2_2: Interbank } \\
\text { Money Market Rates and Indexation Clauses: Interbank market Till } 30 \text { days average - } \\
\text { nominal rate (n) }\end{array}$ & 1992,11 & 2003,12 \\
\hline
\end{tabular}

Panel B: Long-term corporate loan rates

\begin{tabular}{|c|c|c|c|c|c|}
\hline Estonia & 5 & $\mathrm{CB}$ & $\begin{array}{l}\text { 6.1.1 Weighted average annual interest rates of kroon loans by groups of customers and } \\
\text { maturity: long-term kroon loans -commercial undertakings }\end{array}$ & 1997,04 & 2003,12 \\
\hline Hungary & 5 & $\mathrm{CB}$ & $\begin{array}{l}\text { 1. \& 6. Table: Monthly average agreed interest rate of loans and deposits to non-financial } \\
\text { corporations weighted by the amount of new business 1) 2) 3) 4) -- Loans with a maturity } \\
\text { over one vear }\end{array}$ & 1995,01 & 2003,12 \\
\hline Latvia & 5 & $\mathrm{CB}$ & $\begin{array}{l}\text { Weighted average interest rates charged by banks in transactions with domestic enterprises } \\
(\%) \text { : table } 17 \mathrm{~b} \text { - LATOS / IN LATS - new loans -- Long-term }\end{array}$ & 1999,01 & 2003,12 \\
\hline \multirow[t]{2}{*}{ Lithuania } & 5,01 & $\mathrm{CB}$ & corporate loans -1 to 5 years & 1999,01 & 2003,12 \\
\hline & 5,02 & $\mathrm{CB}$ & corporate loans - over 5 years & 1999,01 & 2003,12 \\
\hline \multirow[t]{2}{*}{ Poland } & 5,01 & CB & Loans in zloty to non-financial corporations - with an original maturity of 3 years perioc & 1996,12 & 2003,12 \\
\hline & 5,02 & $\mathrm{CB}$ & Loans in zloty to non-financial corporations - with an original maturity of over 5 years period & 1998,01 & 2003,12 \\
\hline
\end{tabular}

Slovenia $\quad 5 \quad$ CB Average Commercial Banks Interest Rates -- 1. Average Commercial Banks Interest Rates: $1992,012003,12$ (Tolar Indexation Clause) - T2 44 - nominal rate (n): Long term loans -For capital assets

Panel C: Short-term corporate loan rate

\begin{tabular}{|c|c|c|c|c|c|}
\hline Czech Republic & 4 & $\mathrm{CB}$ & $\begin{array}{l}\text { Money and banking statistics }>>>\text { National money and banking statistics }>>>\text { Interest rates } \\
>>>\text { Loans }>>>\text { Client }>>>\text { Newly drawn }>>>\text { Sector breakdown }>>>\text { Monthly: Newly } \\
\text { drawn client loans by sector }(\mathrm{CZK}) \text { - lending rate - Non-financial corporations }(\% \text { : }\end{array}$ & 2001,01 & 2003,12 \\
\hline Estonia & 4 & $\mathrm{CB}$ & $\begin{array}{l}\text { 6.1.1 Weighted average annual interest rates of kroon loans by groups of customers and } \\
\text { maturity - short-term kroon loans -commercial undertakings }\end{array}$ & 1997,04 & 2003,12 \\
\hline Hungary & 4 & $\mathrm{CB}$ & $\begin{array}{l}\text { 1. \& } 6 \text {. Table: Monthly average agreed interest rate of loans and deposits to non-financial } \\
\text { corporations weighted by the amount of new business 1) 2) 3) 4) --- Loans with a maturity up } \\
\text { to one year }\end{array}$ & 1995,01 & 2003,12 \\
\hline Latvia & 4 & $\mathrm{CB}$ & $\begin{array}{l}\text { Table 17b: Weighted average interest rates charged by banks in transactions with domestic } \\
\text { enterprises }(\%) \text { - LATOS / IN LATS - new loans -- short term }\end{array}$ & 1999,01 & 2003,12 \\
\hline Lithuania & 4 & $\mathrm{CB}$ & $\begin{array}{l}\text { corporate loans (authors own calculation as unweighted average of } 4 \text { reported rates with } \\
\text { differing maturities: up to } 1 \mathrm{month}, 1 \text { to } 3 \mathrm{mth}, 3 \text { to } 6 \mathrm{mth} \text {, and } 6 \text { to } 12 \mathrm{mth} \text { ) }\end{array}$ & 1999,01 & 2003,12 \\
\hline Poland & 4 & $\mathrm{CB}$ & Loans in zloty to non-financial corporations - with an original maturity of 1 year perioc & 1996,12 & 2003,12 \\
\hline Slovak Republic & 4 & $\mathrm{CB}$ & $\begin{array}{l}\text { Average Lending Rates of Commercial Banks (\%): 2. NEW LOANS IN TOTAL a) } \\
\text { Enterprise sector - Private sector (incl. cooperatives' }\end{array}$ & 1995,01 & 2003,12 \\
\hline Slovenia & 4 & $\mathrm{CB}$ & $\begin{array}{l}\text { Average Commercial Banks Interest Rates -- 1. Average Commercial Banks Interest Rates: } \\
\text { (Tolar Indexation Clause) - T2 } 42 \text { - nominal rate (n): Short term loans - Working capital }\end{array}$ & 1992,01 & 2003,12 \\
\hline \multicolumn{6}{|c|}{ Panel D: Consumer loan rates } \\
\hline Czech Republic & 3 & $\mathrm{CB}$ & $\begin{array}{l}\text { Money and banking statistics }>>>\text { National money and banking statistics }>>>\text { Interest rates } \\
>>>\text { Loans }>>>\text { Client }>>>\text { Newly drawn }>>>\text { Sector breakdown }>>>\text { Monthly: Newly } \\
\text { drawn client loans by sector (CZK) - ending rate -- Households - individuals }(\%)\end{array}$ & 1993,01 & 2003,12 \\
\hline Estonia & 3 & $\mathrm{CB}$ & $\begin{array}{l}\text { 6.1.4 Weighted average annual interest rates of loans granted to individuals: Consumer } \\
\text { credits - EEK denominated }\end{array}$ & 1997,04 & 2003,12 \\
\hline Hungary & 3 & $\mathrm{CB}$ & $\begin{array}{l}\text { Table } 1 \& 7 \text { : Monthly average agreed interest rate of the loans and deposits to households } \\
\text { weighted by the amount of new business -- Loans for consumtion and other purposes }\end{array}$ & 1995,01 & 2003,12 \\
\hline \multirow[t]{2}{*}{ Latvia } & 3,01 & $\mathrm{CB}$ & $\begin{array}{l}\text { Table 17c: Weighted average interest rates charged by banks in transactions with domestic } \\
\text { privat persons }(\%) \text { - LATOS / IN LATS - new loans - 6-12 months }\end{array}$ & 1999,01 & 2003,12 \\
\hline & 3,02 & $\mathrm{CB}$ & $\begin{array}{l}\text { Table } 17 \mathrm{c} \text { : Weighted average interest rates charged by banks in transactions with domestic } \\
\text { privat persons }(\%) \text { - LATOS / IN LATS - new loans - short term }\end{array}$ & 1999,01 & 2003,12 \\
\hline \multirow[t]{2}{*}{ Lithuania } & 3,01 & CB & months & & 2003,12 \\
\hline & 3,02 & $\mathrm{CB}$ & & & 2003,12 \\
\hline Poland & 3 & $\mathrm{CB}$ & Loans in zloty to households - granted in current accoun1 & 1996,12 & 2003,12 \\
\hline Slovak Republic & 3 & $\mathrm{CB}$ & $\begin{array}{l}\text { Average Lending Rates of Commercial Banks (\%) - 2. NEW LOANS IN TOTAL b) } \\
\text { Households }\end{array}$ & 1995,01 & 2003,12 \\
\hline Slovenia & 3 & $\mathrm{CB}$ & $\begin{array}{l}\text { Average Commercial Banks Interest Rates -- } 1 \text {. Average Commercial Banks Interest Rates: } \\
\text { (Tolar Indexation Clause) - T2 } 43 \text { - nominal rate (n): Short term loans - Consumer credits }\end{array}$ & 1992,01 & 2003,12 \\
\hline \multicolumn{6}{|c|}{ Panel D: Mortgage rates } \\
\hline Estonia & 2 & $\mathrm{CB}$ & $\begin{array}{l}\text { 6.1.4 Weighted average annual interest rates of loans granted to individuals: Housing loans - } \\
\text { EEK denominated }\end{array}$ & 1997,04 & 2003,12 \\
\hline Hungary & 2 & $\mathrm{CB}$ & $\begin{array}{l}\text { Table } 1 . \& 7 \text { : Monthly average agreed interest rate of the loans and deposits to households } \\
\text { weighted by the amount of new business -- Loans for house purchase }\end{array}$ & 1995,01 & 2003,12 \\
\hline
\end{tabular}




\begin{tabular}{|c|c|c|c|c|c|}
\hline \multirow[b]{2}{*}{ country } & \multirow[b]{2}{*}{ 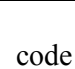 } & \multirow[b]{2}{*}{ source } & \multirow[b]{2}{*}{ description } & \multicolumn{2}{|c|}{ data availability } \\
\hline & & & & to & from \\
\hline Latvia & 2 & $\mathrm{CB}$ & $\begin{array}{l}\text { Table } 17 \mathrm{c} \text { : Weighted average interest rates charged by banks in transactions with domestic } \\
\text { privat persons }(\%) \text { - LATOS / IN LATS - new loans - long-term }\end{array}$ & 1999,01 & 2003,12 \\
\hline \multirow{2}{*}{ Lithuania } & 2,01 & $\mathrm{CB}$ & consumer housing loans - 1 to 5 years & 1999,01 & 2003,12 \\
\hline & 2,02 & $\mathrm{CB}$ & consumer housing loans - over 5 years & 1999,01 & 2003,12 \\
\hline Slovenia & 2 & $\mathrm{CB}$ & $\begin{array}{l}\text { Average Commercial Banks Interest Rates -- } 1 \text {. Average Commercial Banks Interest Rates: } \\
\text { (Tolar Indexation Clause) - T2_4_5 - nominal rate (n): Long term loans - For population - } \\
\text { housing programe }\end{array}$ & 1992,01 & 2003,12 \\
\hline \multicolumn{6}{|c|}{ Panel E: Current account deposit rates } \\
\hline Hungary & 7 & $\mathrm{CB}$ & $\begin{array}{l}\text { Table } 1 . \& \text { 7:Monthly average agreed interest rate of the loans and deposits to households } \\
\text { weighted by the amount of new business }(\%) \text {-- Overnight deposits }\end{array}$ & 1995,01 & 2003,12 \\
\hline Poland & $\begin{array}{l}7,01 \\
7,02 \\
\end{array}$ & $\begin{array}{l}\mathrm{CB} \\
\mathrm{CB}\end{array}$ & $\begin{array}{l}\text { Households, zloty deposits - checking accounts (individuals) } \\
\text { Non-financial corporations, zloty deposits - current accounts }\end{array}$ & $\begin{array}{l}1996,12 \\
1996,12\end{array}$ & $\begin{array}{l}2003,12 \\
2003,12\end{array}$ \\
\hline
\end{tabular}

Panel E: Saving account rates

\begin{tabular}{llllllll}
\hline Czech Republic & 9 & CB & Money and banking statistics $>>>$ National money and banking statistics $>>>$ Interest rates & $1993,01 \quad 2003,12$
\end{tabular}
>>> Deposits $>>>$ Client $>>>$ Maturity breakdown $>>>$ Monthly $\|$ Deposits by time (CZK) Demand deposits $(\%)$

Lithuania $\quad 9 \quad \mathrm{CB}$ Average Annual Interest Rates on Saving deposits in Litas - Per cent; monthly average - $1999,012003,12$ households

Slovak Republic $\quad 9 \quad$ CB Average Interest Rates on Crown Deposits (\%) -- Demand deposits - Total deposits without $1995,012003,12$ REPO -demand deposits

Slovenia $\quad 9 \quad \mathrm{CB} \quad \mathrm{CB}$ of Slovenia Monthly Bulleting: 4. Average Commercial Banks Interest Rates -- $1.1992,012003,12$ Average Commercial Banks Interest Rates: (Tolar Indexation Clause) - T2_4_1 (series n): Demand deposits

Panel E: Time deposit rates

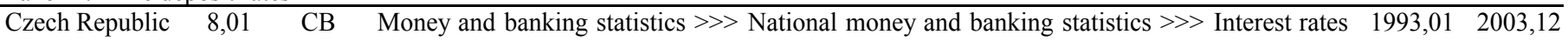
>>> Deposits $\gg>>$ Client $\gg>>$ Maturity breakdown $\gg>$ M Monthly || Deposits by time (CZK): Short-term (up to 1 vear) $(\%)$

8,02 CB Money and banking statistics $>>>$ National money and banking statistics $>>>$ Interest rates $1993,012003,12$ >> Deposits $>>>$ Client $\gg>>$ Maturity breakdown $>>>$ Monthly || Deposits by time (CZK): Over 1 vear and up to 4 vears $(\%)$

8,03 CB Money and banking statistics $\gg>>$ National money and banking statistics $\gg>>$ Interest rates $1993,01 \quad 2003,12$ >> Deposits $\gg>$ C Client $\gg>>$ Maturity breakdown $\gg>>$ Monthly || Deposits by time (CZK): Over 4 vears $(\%)$

8,04 CB Money and banking statistics $\gg>>$ National money and banking statistics $\gg>$ Interest rates $1993,01 \quad 2003,12$ >>> Deposits $\gg>>$ Client $\gg>>$ Maturity breakdown $\gg>>$ Monthly || Deposits by time (CZK): Time deposits (\%)

Estonia $\quad 8,01 \quad \mathrm{CB} \quad$ 6.2.1 Weighted average annual interest rates of kroon time deposits by groups of customers and maturities -- TIME DEPOSITS TOTAL: commercial undertakings

\begin{tabular}{|c|c|c|c|c|c|}
\hline \multirow[t]{6}{*}{ tovera } & . & - & and maturities -- TIME DEPOSITS TOTAL: commercial undertakings & 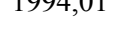 & (1) \\
\hline & 8,02 & $\mathrm{CB}$ & $\begin{array}{l}\text { 6.2.1 Weighted average annual interest rates of kroon time deposits by groups of customers } \\
\text { and maturities -- TIME DEPOSITS TOTAL: individuals }\end{array}$ & 1994,01 & 2003,12 \\
\hline & 8,03 & $\mathrm{CB}$ & $\begin{array}{l}\text { 6.2.1 Weighted average annual interest rates of kroon time deposits by groups of customers } \\
\text { and maturities -- TIME DEPOSITS TOTAL: up to } 3 \text { months }\end{array}$ & 1994,01 & 2003,12 \\
\hline & 8,04 & $\mathrm{CB}$ & $\begin{array}{l}\text { 6.2.1 Weighted average annual interest rates of kroon time deposits by groups of customers } \\
\text { and maturities -- TIME DEPOSITS TOTAL: } 3 \text { - } 6 \text { months }\end{array}$ & 1997,04 & 2003,12 \\
\hline & 8,05 & $\mathrm{CB}$ & $\begin{array}{l}\text { 6.2.1 Weighted average annual interest rates of kroon time deposits by groups of customers } \\
\text { and maturities -- TIME DEPOSITS TOTAL: } 6 \text { - } 12 \text { months }\end{array}$ & 1994,01 & 2003,12 \\
\hline & 8,06 & $\mathrm{CB}$ & $\begin{array}{l}\text { 6.2.1 Weighted average annual interest rates of kroon time deposits by groups of customers } \\
\text { and maturities -- TIME DEPOSITS TOTAL: over } 1 \text { year }\end{array}$ & 1994,01 & 2003,12 \\
\hline \multirow[t]{2}{*}{ Hungary } & 8,01 & $\mathrm{CB}$ & $\begin{array}{l}\text { 1. \& } 7 \text { table - Monthly average agreed interest rate of the loans and deposits to households } \\
\text { weighted by the amount of new business (\%): deposits - up to } 1 \text { yeal }\end{array}$ & 1995,01 & 2003,12 \\
\hline & 8,02 & $\mathrm{CB}$ & $\begin{array}{l}\text { 1. \& } 7 \text { table - Monthly average agreed interest rate of the loans and deposits to households } \\
\text { weighted by the amount of new business }(\%) \text { : deposits over } 1 \text { yeal }\end{array}$ & 1995,01 & 2003,12 \\
\hline \multirow[t]{9}{*}{ Latvia } & 8,01 & $\mathrm{CB}$ & $\begin{array}{l}\text { Table } 17 \mathrm{a} \text { : Weighted average interest rates charged by banks in transactions with domestic } \\
\text { enterprises and private persons, }(\%) \text {, in latos (lats): Up to } 1 \text { month }\end{array}$ & 1999,01 & 2003,12 \\
\hline & 8,02 & $\mathrm{CB}$ & $\begin{array}{l}\text { Table 17a: Weighted average interest rates charged by banks in transactions with domestic } \\
\text { enterprises and private persons, }(\%) \text {, in latos (lats) - New deposits : } 1-3 \text { months }\end{array}$ & 1999,01 & 2003,12 \\
\hline & 8,03 & $\mathrm{CB}$ & $\begin{array}{l}\text { Table 17a: Weighted average interest rates charged by banks in transactions with domestic } \\
\text { enterprises and private persons, }(\%) \text {, in latos (lats) - New deposits : } 3-6 \text { months }\end{array}$ & 1999,01 & 2003,12 \\
\hline & 8,04 & $\mathrm{CB}$ & $\begin{array}{l}\text { Table 17a: Weighted average interest rates charged by banks in transactions with domestic } \\
\text { enterprises and private persons, }(\%) \text {, in latos (lats) - New deposits : } 6-12 \text { months }\end{array}$ & 1999,01 & 2003,12 \\
\hline & 8,05 & $\mathrm{CB}$ & $\begin{array}{l}\text { Table 17a: Weighted average interest rates charged by banks in transactions with domestic } \\
\text { enterprises and private persons, }(\%) \text {, in latos (lats) - New deposits : Short-term }\end{array}$ & 1999,01 & 2003,12 \\
\hline & 8,06 & $\mathrm{CB}$ & $\begin{array}{l}\text { Table 17a: Weighted average interest rates charged by banks in transactions with domestic } \\
\text { enterprises and private persons, }(\%) \text {, in latos (lats) - New deposits : Long-term }\end{array}$ & 1999,01 & 2003,12 \\
\hline & 8,07 & $\mathrm{CB}$ & $\begin{array}{l}\text { Table } 17 \mathrm{~b} \text { : Weighted average interest rates charged by banks in transactions with domestic } \\
\text { enterprises, }(\%) \text {, in latos - New deposits : Up to } 1 \text { month }\end{array}$ & 1999,01 & 2003,12 \\
\hline & 8,08 & $\mathrm{CB}$ & $\begin{array}{l}\text { Table } 17 \mathrm{~b} \text { : Weighted average interest rates charged by banks in transactions with domestic } \\
\text { enterprises, }(\%) \text {, in latos - New deposits : } 1-3 \text { months }\end{array}$ & 1999,01 & 2003,12 \\
\hline & 8,09 & $\mathrm{CB}$ & $\begin{array}{l}\text { Table } 17 \mathrm{~b} \text { : Weighted average interest rates charged by banks in transactions with domestic } \\
\text { enterprises, }(\%) \text {, in latos - New deposits : } 3-6 \text { months }\end{array}$ & 1999,01 & 2003,12 \\
\hline
\end{tabular}




\begin{tabular}{|c|c|c|c|c|c|}
\hline \multirow{2}{*}{ country } & \multirow[b]{2}{*}{ code } & \multirow[b]{2}{*}{ source } & \multirow[b]{2}{*}{ description } & \multicolumn{2}{|c|}{ data availability } \\
\hline & & & & to & from \\
\hline \multirow{24}{*}{ Lithuania } & 8,10 & $\mathrm{CB}$ & $\begin{array}{l}\text { Table 17b: Weighted average interest rates charged by banks in transactions with domestic } \\
\text { enterprises, }(\%) \text {, in latos - New deposits : } 6-12 \text { months }\end{array}$ & 1999,01 & 2003,12 \\
\hline & 8,11 & $\mathrm{CB}$ & $\begin{array}{l}\text { Table 17b: Weighted average interest rates charged by banks in transactions with domestic } \\
\text { enterprises, }(\%) \text {, in latos - New deposits : Short-term }\end{array}$ & 1999,01 & 2003,12 \\
\hline & 8,12 & $\mathrm{CB}$ & $\begin{array}{l}\text { Table 17b: Weighted average interest rates charged by banks in transactions with domestic } \\
\text { enterprises, }(\%) \text {, in latos, new deposits - New deposits : Long-term }\end{array}$ & 1999,01 & 2003,12 \\
\hline & 8,13 & $\mathrm{CB}$ & $\begin{array}{l}\text { Table } 17 \mathrm{c} \text { : Weighted average interest rates charged by banks in transactions with domestic } \\
\text { private persons, }(\%) \text {, in latos - New deposits : Up to } 1 \text { month }\end{array}$ & 1999,01 & 2003,12 \\
\hline & 8,14 & $\mathrm{CB}$ & $\begin{array}{l}\text { Table } 17 \mathrm{c} \text { : Weighted average interest rates charged by banks in transactions with domestic } \\
\text { private persons, }(\%) \text {, in latos - New deposits : } 1-3 \text { months }\end{array}$ & 1999,01 & 2003,12 \\
\hline & 8,15 & $\mathrm{CB}$ & $\begin{array}{l}\text { Table } 17 \mathrm{c} \text { : Weighted average interest rates charged by banks in transactions with domestic } \\
\text { private persons, }(\%) \text {, in latos - New deposits : 3-6 months }\end{array}$ & 1999,01 & 2003,12 \\
\hline & 8,16 & $\mathrm{CB}$ & $\begin{array}{l}\text { Table } 17 \mathrm{c} \text { : Weighted average interest rates charged by banks in transactions with domestic } \\
\text { private persons, }(\%) \text {, in latos - New deposits : } 6-12 \text { months }\end{array}$ & 1999,01 & 2003,12 \\
\hline & 8,17 & $\mathrm{CB}$ & $\begin{array}{l}\text { Table } 17 \mathrm{c} \text { : Weighted average interest rates charged by banks in transactions with domestic } \\
\text { private persons, }(\%) \text {, in latos - New deposits Short-term }\end{array}$ & 1999,01 & 2003,12 \\
\hline & 8,18 & $\mathrm{CB}$ & $\begin{array}{l}\text { Table } 17 \mathrm{c} \text { : Weighted average interest rates charged by banks in transactions with domestic } \\
\text { private persons, }(\%) \text {, in latos - New deposits Long-term }\end{array}$ & 1999,01 & 2003,12 \\
\hline & 8,01 & $\mathrm{CB}$ & $\begin{array}{l}\text { Average Annual Interest Rates on Time deposits in Litas - Per cent; monthly average: up to } 1 \\
\text { month - total amount (=all investors) }\end{array}$ & 1992,12 & 2003,12 \\
\hline & 8,02 & $\mathrm{CB}$ & $\begin{array}{l}\text { Average Annual Interest Rates on Time deposits in Litas - Per cent; monthly average: up to } 1 \\
\text { month - households }\end{array}$ & 1999,01 & 2003,12 \\
\hline & 8,03 & $\mathrm{CB}$ & $\begin{array}{l}\text { Average Annual Interest Rates on Time deposits in Litas - Per cent; monthly average: } 1 \text { - } 3 \\
\text { months - total amount (=all investors) }\end{array}$ & 1992,12 & 2003,12 \\
\hline & 8,04 & $\mathrm{CB}$ & $\begin{array}{l}\text { Average Annual Interest Rates on Time deposits in Litas - Per cent; monthly average: } 1 \text { - } 3 \\
\text { months - households }\end{array}$ & 1999,01 & 2003,12 \\
\hline & 8,05 & $\mathrm{CB}$ & $\begin{array}{l}\text { Average Annual Interest Rates on Time deposits in Litas - Per cent; monthly average: } 3 \text { - } 6 \\
\text { months - total amount (=all investors) }\end{array}$ & 1992,12 & 2003,12 \\
\hline & 8,06 & $\mathrm{CB}$ & $\begin{array}{l}\text { Average Annual Interest Rates on Time deposits in Litas - Per cent; monthly average: } 3 \text { - } 6 \\
\text { months - households }\end{array}$ & 1999,01 & 2003,12 \\
\hline & 8,07 & $\mathrm{CB}$ & $\begin{array}{l}\text { Average Annual Interest Rates on Time deposits in Litas - Per cent; monthly average: } 6 \text { - } 12 \\
\text { months total amount (=all investors) }\end{array}$ & 1992,12 & 2003,12 \\
\hline & 8,08 & $\mathrm{CB}$ & $\begin{array}{l}\text { Average Annual Interest Rates on Time deposits in Litas - Per cent; monthly average: } 6 \text { - } 12 \\
\text { months - households }\end{array}$ & 1999,01 & 2003,12 \\
\hline & 8,09 & $\mathrm{CB}$ & $\begin{array}{l}\text { Average Annual Interest Rates on Time deposits in Litas - Per cent; monthly average: } 1 \text { - } 2 \\
\text { years**total amount (=all investors) }\end{array}$ & 1992,12 & 2003,12 \\
\hline & 8,10 & $\mathrm{CB}$ & $\begin{array}{l}\text { Average Annual Interest Rates on Time deposits in Litas - Per cent; monthly average: } 1 \text { - } 2 \\
\text { years** - households }\end{array}$ & 1999,01 & 2003,12 \\
\hline & 8,11 & $\mathrm{CB}$ & $\begin{array}{l}\text { Average Annual Interest Rates on Time deposits in Litas - Per cent; monthly average: over } 2 \\
\text { years total amount (=all investors) }\end{array}$ & 1997,01 & 2003,12 \\
\hline & 8,12 & $\mathrm{CB}$ & $\begin{array}{l}\text { Average Annual Interest Rates on Time deposits in Litas - Per cent; monthly average: over } 2 \\
\text { years - households }\end{array}$ & 1999,01 & 2003,12 \\
\hline & 8,13 & $\mathrm{CB}$ & $\begin{array}{l}\text { Average Annual Interest Rates on Time deposits in Litas - Per cent; monthly average: total- } \\
\text { total amount (=all investors) }\end{array}$ & 1992,12 & 2003,12 \\
\hline & 8,14 & $\mathrm{CB}$ & $\begin{array}{l}\text { Average Annual Interest Rates on Time deposits in Litas - Per cent; monthly average: total - } \\
\text { households }\end{array}$ & 1999,01 & 2003,12 \\
\hline & 8,15 & $\mathrm{CB}$ & Average Annual Interest Rates on demand deposits in Litas - Per cent; monthly averag, & 1992,12 & 2003,12 \\
\hline \multirow[t]{12}{*}{ Poland } & 8,01 & $\mathrm{CB}$ & Households, zloty deposits - time deposits with agreed maturity of 1 montl & 1999,01 & 2003,12 \\
\hline & 8,02 & $\mathrm{CB}$ & Households, zloty deposits - time deposits with agreed maturity of 3 month: & 1996,12 & 2003,12 \\
\hline & 8,03 & $\mathrm{CB}$ & Households, zloty deposits - time deposits with agreed maturity of 6 month: & 1996,12 & 2003,12 \\
\hline & 8,04 & $\mathrm{CB}$ & Households, zloty deposits - time deposits with agreed maturity of 1 yea & 1996,12 & 2003,12 \\
\hline & 8,05 & $\mathrm{CB}$ & Households, zloty deposits - time deposits with agreed maturity of 2 year: & 1996,12 & 2003,12 \\
\hline & 8,06 & $\mathrm{CB}$ & Personal sector, zloty deposits - 36-month & 1996,12 & 2002,02 \\
\hline & 8,07 & $\mathrm{CB}$ & Non-financial corporations, zloty deposits - time deposits with agreed maturity of 1 montl & 1996,12 & 2003,12 \\
\hline & 8,08 & $\mathrm{CB}$ & Non-financial corporations, zloty deposits - time deposits with agreed maturity of 3 month: & 1996,12 & 2003,12 \\
\hline & 8,09 & $\mathrm{CB}$ & Non-financial corporations, zloty deposits - time deposits with agreed maturity of 6 month: & 1996,12 & 2003,12 \\
\hline & 8,10 & $\mathrm{CB}$ & Non-financial corporations, zloty deposits - time deposits with agreed maturity of 1 yea & 1996,12 & 2003,12 \\
\hline & 8,11 & $\mathrm{CB}$ & Non-financial corporations, zloty deposits - time deposits with agreed maturity of 2 year: & 1996,12 & 2003,12 \\
\hline & 8,12 & $\mathrm{CB}$ & Corporate sector, zloty deposits - 36-month & 1996,12 & 2002,12 \\
\hline \multirow[t]{6}{*}{ Slovak Republic } & 8,01 & $\mathrm{CB}$ & $\begin{array}{l}\text { Average Interest Rates on Crown Deposits (\%) - Total deposits without REPO - term } \\
\text { deposits - - 1-month deposits }\end{array}$ & 1995,01 & 2003,12 \\
\hline & 8,02 & $\mathrm{CB}$ & $\begin{array}{l}\text { Average Interest Rates on Crown Deposits (\%) - Total deposits without REPO -term deposits } \\
\text { - 3-month deposits }\end{array}$ & 1995,01 & 2003,12 \\
\hline & 8,03 & $\mathrm{CB}$ & $\begin{array}{l}\text { Average Interest Rates on Crown Deposits (\%) - Total deposits without REPO - term deposits } \\
\text { - - 6-month deposits }\end{array}$ & 1995,01 & 2003,12 \\
\hline & 8,04 & $\mathrm{CB}$ & $\begin{array}{l}\text { Average Interest Rates on Crown Deposits (\%) - Total deposits without REPO -term deposits } \\
\text { - 1-year deposits }\end{array}$ & 1995,01 & 2003,12 \\
\hline & 8,05 & $\mathrm{CB}$ & $\begin{array}{l}\text { Average Interest Rates on Crown Deposits (\%) - Total deposits without REPO -term deposits } \\
\text { - 2-year deposits }\end{array}$ & 1995,04 & 2003,12 \\
\hline & 8,06 & $\mathrm{CB}$ & $\begin{array}{l}\text { Average Interest Rates on Crown Deposits (\%) - Total deposits without REPO -term deposits } \\
\text { - 5-year deposits }\end{array}$ & 1995,01 & 2003,12 \\
\hline
\end{tabular}




\begin{tabular}{|c|c|c|c|c|c|}
\hline \multirow[b]{2}{*}{ country } & \multirow[b]{2}{*}{ code } & \multirow[b]{2}{*}{ source } & \multirow[b]{2}{*}{ description } & \multicolumn{2}{|c|}{ data availability } \\
\hline & & & & to & from \\
\hline \multirow{6}{*}{ Slovenia } & 8,07 & CB & $\begin{array}{l}\text { Average Interest Rates on Crown Deposits (\%) - Total deposits without REPO -term deposits } \\
\text { - over } 5 \text { year }\end{array}$ & 1995,01 & 2003,12 \\
\hline & 8,01 & $\mathrm{CB}$ & $\begin{array}{l}\mathrm{CB} \text { of Slovenia Monthly Bulleting: 4. Average Commercial Banks Interest Rates -- } 1 . \\
\text { Average Commercial Banks Interest Rates: (Tolar Indexation Clause) - T2_4_1 (series n): } \\
\text { Time deposits - Till } 30 \text { davs }\end{array}$ & 1992,01 & 2003,12 \\
\hline & 8,02 & $\mathrm{CB}$ & $\begin{array}{l}\text { CB of Slovenia Monthly Bulleting: 4. Average Commercial Banks Interest Rates -- } 1 \text {. } \\
\text { Average Commercial Banks Interest Rates: (Tolar Indexation Clause) }-T_{-}{ }_{-}{ }_{-}(\text {(series } \mathrm{n}) \text { : } \\
\text { Time deposits }-32-90 \text { davs }\end{array}$ & 1992,01 & 2003,12 \\
\hline & 8,03 & $\mathrm{CB}$ & $\begin{array}{l}\text { CB of Slovenia Monthly Bulleting: 4. Average Commercial Banks Interest Rates -- } 1 \text {. } \\
\text { Average Commercial Banks Interest Rates: (Tolar Indexation Clause) }- \text { T2_4_1 (series n): } \\
\text { Time deposits }-92-180 \text { days }\end{array}$ & 1992,01 & 2003,12 \\
\hline & 8,04 & $\mathrm{CB}$ & $\begin{array}{l}\text { CB of Slovenia Monthly Bulleting: 4. Average Commercial Banks Interest Rates -- } 1 . \\
\text { Average Commercial Banks Interest Rates: (Tolar Indexation Clause) }-T 2 \_4 \_1 \text { (series n): }\end{array}$ & 1992,01 & 2003,12 \\
\hline & 8,05 & $\mathrm{CB}$ & $\begin{array}{l}\text { Time deposits }-182 \text { davs }-1 \text { vear } \\
\text { CB of Slovenia Monthly Bulleting: 4. Average Commercial Banks Interest Rates -- } 1 . \\
\text { Average Commercial Banks Interest Rates: (Tolar Indexation Clause) }-T_{2}{ }_{-} \_1 \text { (series n): } \\
\text { Time deposits -Over } 1 \text { vear }\end{array}$ & 1992,01 & 2003,12 \\
\hline
\end{tabular}

Note: IFS refers to the International Financial Statistics. CB indicates that the data was obtained from the national central bank's website. ${ }^{1}$ Data is missing from June to September $1997 .^{2}$ The rate is the same as the one provided by IFS. 


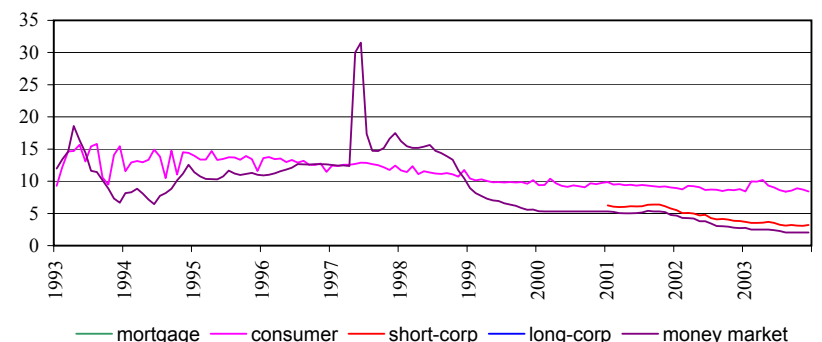

Panel C: Loan rates in Estonia

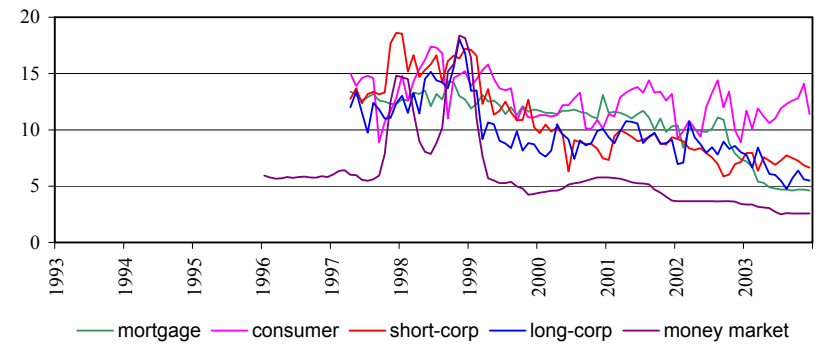

Panel E: Loan rates in Hungary

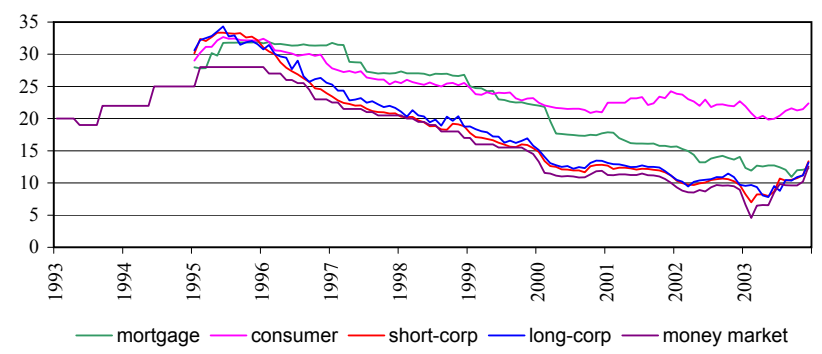

Panel G: Loan rates in Latvia

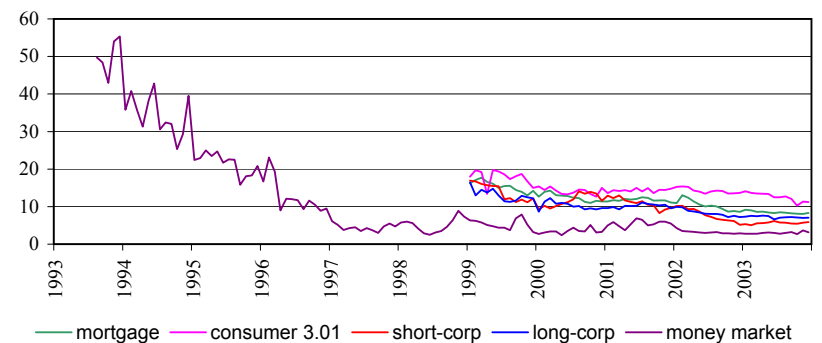

Panel I: Loan rates in Lithuania

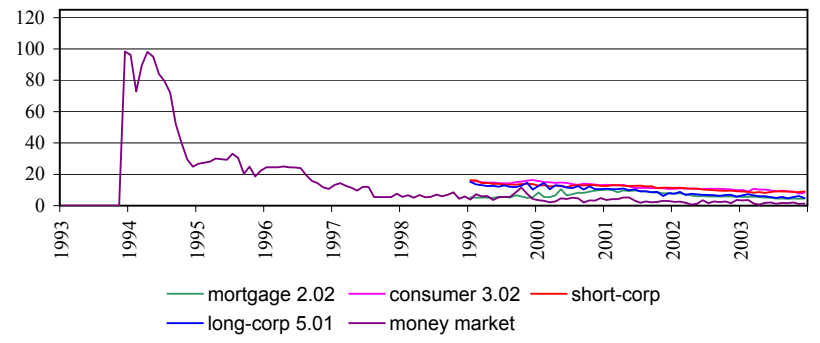

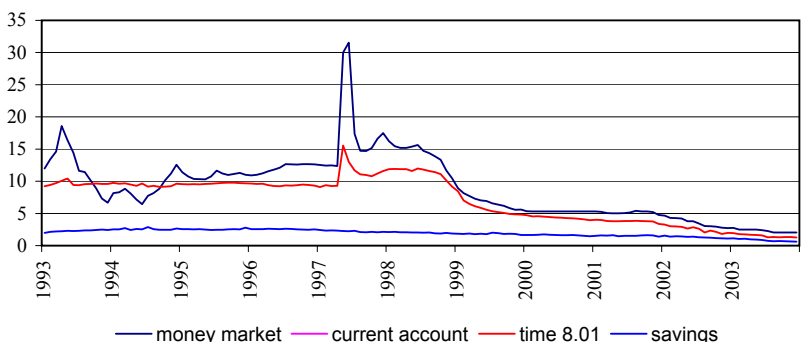

Panel D: Deposit rates in Estonia

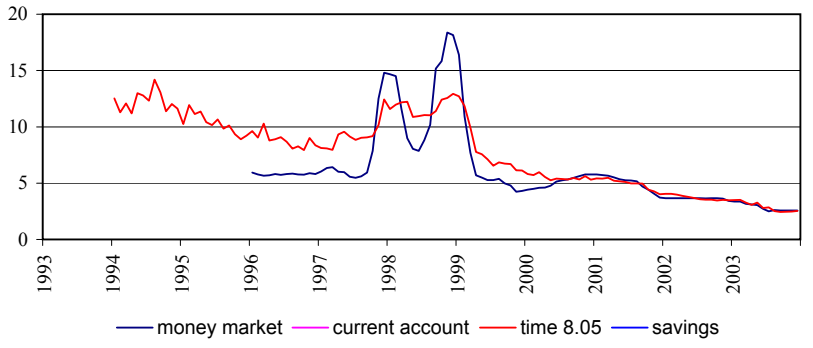

Panel F: Deposit rates in Hungary

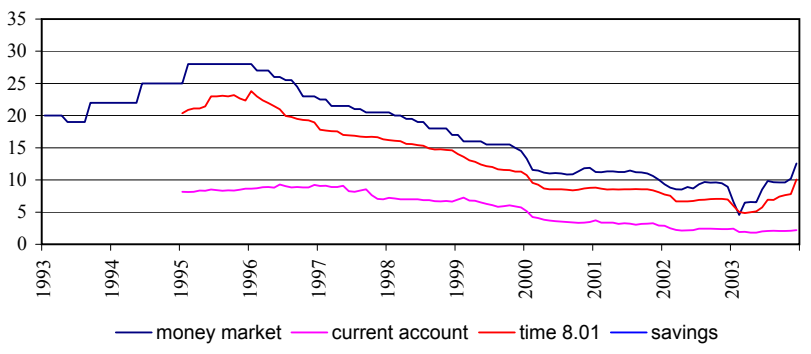

Panel H: Deposit rates in Latvia

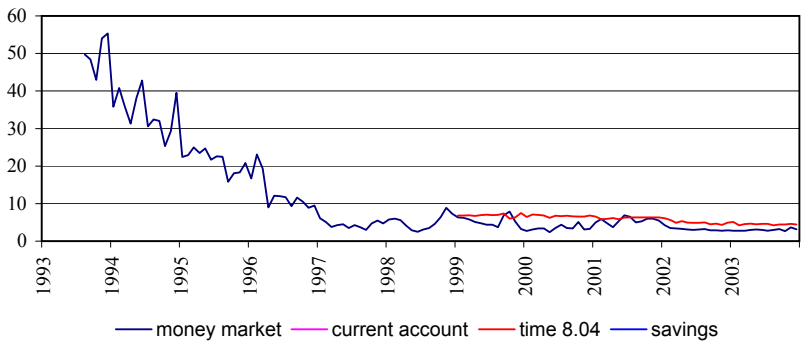

Panel J: Deposit rates in Lithuania

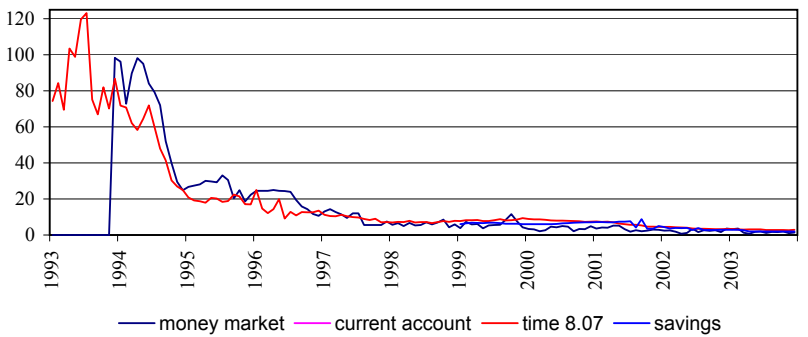




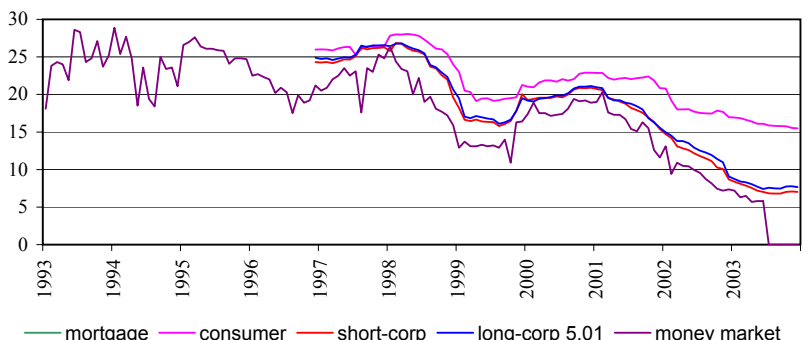

Panel M: Loan rates in the Slovak Republic

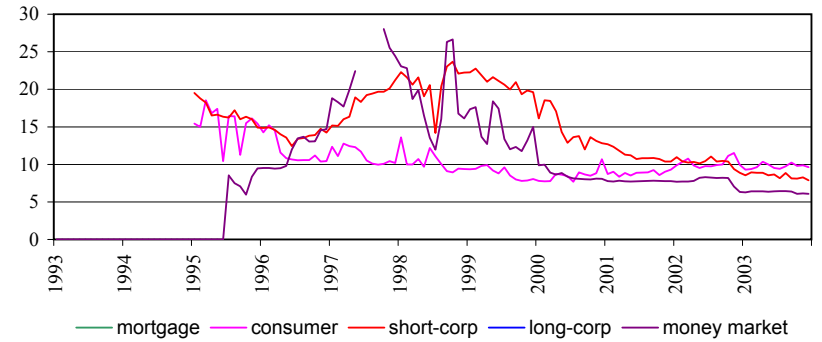

Panel O: Loan rates in Slovenia

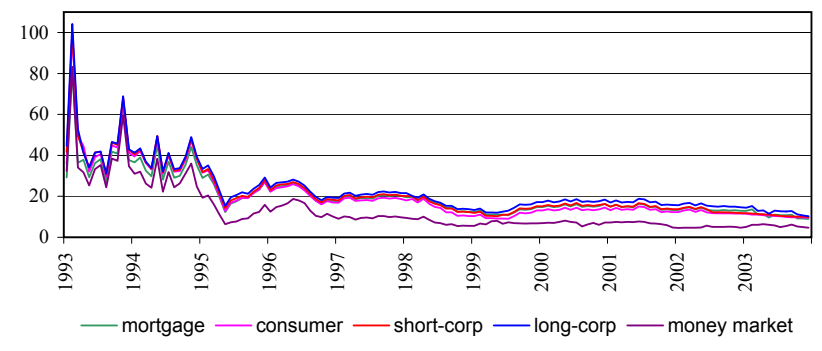

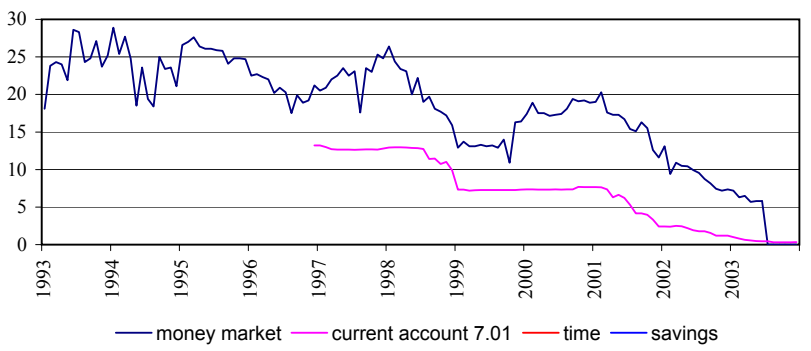

Panel N: Deposit rates in the Slovak Republic

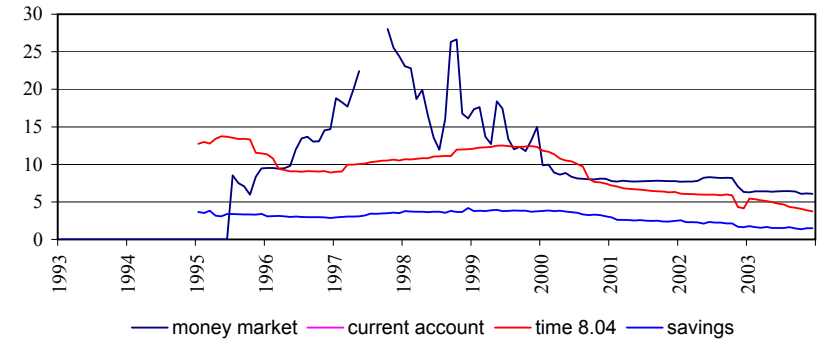

Panel P: Deposit rates in Slovenia

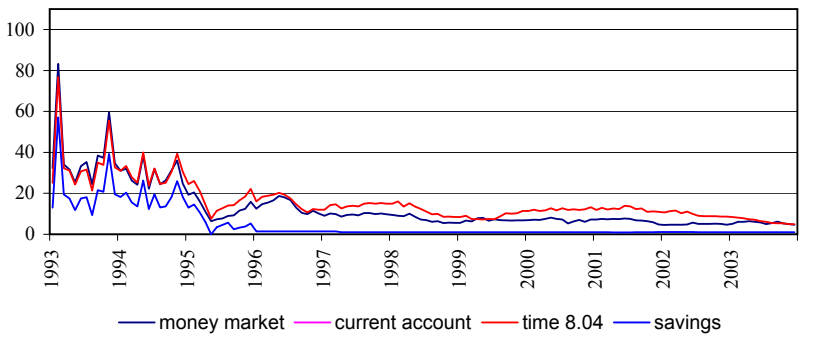


monetary policy rate

Can cointe-

bank rate

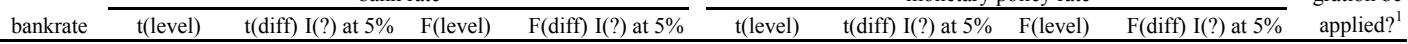
country bankrate t(level)

\begin{tabular}{|c|c|c|c|c|c|c|c|c|c|c|c|c|c|c|}
\hline \multicolumn{15}{|c|}{ Panel A: Short-term corporate loan rates } \\
\hline Czech Republic & 4 & $-1,99$ & $-3,27$ & 1 & 1,97 & 5,41 & 2 & $-3,67$ & $-11,82$ & 0 & 6,75 & 69,84 & 0 & yes \\
\hline Estonia & 4 & $-2,83$ & $-7,35$ & 1 & 4,03 & 26,99 & 1 & $-4,10$ & $-4,53$ & 0 & 8,46 & 10,25 & 0 & yes \\
\hline Hungary & 4 & 0,55 & $-5,04$ & 1 & 4,08 & 13,26 & 1 & $-2,31$ & $-7,95$ & 1 & 3,25 & 31,72 & 1 & yes \\
\hline Latvia & 4 & $-2,04$ & $-4,46$ & 1 & 2,53 & 9,93 & 1 & $-2,78$ & $-15,47$ & 1 & 5,48 & 119,65 & 1 & yes \\
\hline Lithuania & 4 & $-3,15$ & $-8,53$ & 0 & 5,24 & 36,62 & 1 & $-3,81$ & $-8,13$ & 0 & 10,23 & 33,53 & 0 & yes \\
\hline Poland & 4 & $-1,84$ & $-3,81$ & 1 & 2,03 & 7,28 & 1 & $-2,67$ & $-11,17$ & 1 & 3,95 & 62,82 & 1 & yes \\
\hline Slovak Republic & 4 & $-1,32$ & $-8,15$ & 1 & 1,02 & 33,24 & 1 & $-3,45$ & $-9,85$ & 0 & 6,20 & 48,58 & 1 & yes \\
\hline Slovenia & 4 & $-8,67$ & $-13,50$ & 0 & 41,76 & 91,29 & 0 & $-5,14$ & $-15,27$ & 0 & 14,54 & 117,08 & 0 & no \\
\hline \multicolumn{15}{|c|}{ Panel B: Long-term corporate loan rates } \\
\hline Estonia & 5 & $-2,96$ & $-8,15$ & 0 & 4,39 & 33,22 & 1 & $-4,10$ & $-4,53$ & 0 & 8,46 & 10,25 & 0 & yes \\
\hline Hungary & 5 & 0,64 & $-6,65$ & 1 & 2,78 & 22,63 & 1 & $-2,31$ & $-7,95$ & 1 & 3,25 & 31,72 & 1 & yes \\
\hline Latvia & 5 & $-3,10$ & $-7,29$ & 0 & 4,98 & 26,54 & 1 & $-2,78$ & $-15,47$ & 1 & 5,48 & 119,65 & 1 & yes \\
\hline \multirow[t]{2}{*}{ Lithuania } & 5,01 & $-4,57$ & $-12,18$ & 0 & 10,50 & 74,35 & 0 & $-3,81$ & $-8,13$ & 0 & 10,23 & 33,53 & 0 & no \\
\hline & 5,02 & $-3,50$ & $-8,79$ & 0 & 6,65 & 38,80 & 0 & $-3,81$ & $-8,13$ & 0 & 10,23 & 33,53 & 0 & no \\
\hline \multirow[t]{2}{*}{ Poland } & 5,01 & $-1,91$ & $-3,91$ & 1 & 2,10 & 7,67 & 1 & $-2,67$ & $-11,17$ & 1 & 3,95 & 62,82 & 1 & yes \\
\hline & 5,02 & $-1,23$ & $-4,11$ & 1 & 0,76 & 8,47 & 1 & $-2,67$ & $-11,17$ & 1 & 3,95 & 62,82 & 1 & yes \\
\hline Slovenia & 5 & $-8,71$ & $-13,83$ & 0 & 42,17 & 95,83 & 0 & $-5,14$ & $-15,27$ & 0 & 14,54 & 117,08 & 0 & no \\
\hline Panel C: Consum & oan rat & & & & & & & & & & & & & \\
\hline Czech Republic & 3 & $-7,71$ & $-14,59$ & 0 & 29,89 & 106,63 & 0 & $-3,67$ & $-11,82$ & 0 & 6,75 & 69,84 & 0 & no \\
\hline Estonia & 3 & $-3,66$ & $-7,48$ & 0 & 6,69 & 28,00 & 0 & $-4,10$ & $-4,53$ & 0 & 8,46 & 10,25 & 0 & no \\
\hline Hungary & 3 & $-1,31$ & $-7,07$ & 1 & 1,17 & 25,40 & 1 & $-2,31$ & $-7,95$ & 1 & 3,25 & 31,72 & 1 & yes \\
\hline Latvia & 3,01 & $-3,31$ & $-9,29$ & 0 & 5,55 & 43,20 & 1 & $-2,78$ & $-15,47$ & 1 & 5,48 & 119,65 & 1 & yes \\
\hline & 3,02 & $-4,77$ & $-7,58$ & 0 & 11,47 & 28,70 & 0 & $-2,78$ & $-15,47$ & 1 & 5,48 & 119,65 & 1 & no \\
\hline Lithuania & 3,01 & $-6,25$ & $-9,16$ & 0 & 19,54 & 41,99 & 0 & $-3,81$ & $-8,13$ & 0 & 10,23 & 33,53 & 0 & no \\
\hline & 3,02 & $-2,49$ & $-5,43$ & 1 & 3,16 & 14,74 & 1 & $-3,81$ & $-8,13$ & 0 & 10,23 & 33,53 & 0 & yes \\
\hline Poland & 3 & $-1,86$ & $-4,23$ & 1 & 1,82 & 8,97 & 1 & $-2,67$ & $-11,17$ & 1 & 3,95 & 62,82 & 1 & yes \\
\hline Slovak Republic & 3 & $-3,06$ & $-12,59$ & 0 & 4,94 & 79,35 & 1 & $-3,45$ & $-9,85$ & 0 & 6,20 & 48,58 & 1 & yes \\
\hline Slovenia & 3 & $-8,72$ & $-13,86$ & 0 & 42,13 & 96,31 & 0 & $-5,14$ & $-15,27$ & 0 & 14,54 & 117,08 & 0 & no \\
\hline Panel D: Mortgag & ates & & & & & & & & & & & & & \\
\hline Estonia & 2 & $-1,45$ & $\begin{array}{l}-7,63 \\
\end{array}$ & 1 & 1,97 & 29,11 & 1 & $-4,10$ & $-4,53$ & 0 & 8,46 & 10,25 & 0 & yes \\
\hline Hungary & 2 & $-4,21$ & $-6,32$ & 0 & 9,71 & 20,00 & 0 & $-2,31$ & $-7,95$ & 1 & 3,25 & 31,72 & 1 & yes \\
\hline Latvia & 2 & $-2,87$ & $-7,28$ & 1 & 4,52 & 26,72 & 1 & $-2,78$ & $-15,47$ & 1 & 5,48 & 119,65 & 1 & yes \\
\hline Lithuania & 2,01 & $-3,17$ & $-9,03$ & 0 & 5,03 & 40,77 & 1 & $-3,81$ & $-8,13$ & 0 & 10,23 & 33,53 & 0 & yes \\
\hline & 2,02 & $-1,76$ & $-9,17$ & 1 & 2,38 & 42,05 & 1 & $-3,81$ & $-8,13$ & 0 & 10,23 & 33,53 & 0 & yes \\
\hline Slovenia & 2 & $-8,90$ & $-13,89$ & 0 & 43,61 & 96,71 & 0 & $-5,14$ & $-15,27$ & 0 & 14,54 & 117,08 & 0 & no \\
\hline Panel E: Current & ount de & rates & & & & & & & & & & & & \\
\hline Hungary & 7 & $-2,48$ & $\begin{array}{l}-6,94 \\
\end{array}$ & 1 & 3,20 & 24,11 & 1 & $-2,31$ & $-7,95$ & 1 & 3,25 & 31,72 & 1 & yes \\
\hline Poland & 7,01 & $-2,10$ & $-5,58$ & 1 & 2,20 & 15,55 & 1 & $-2,67$ & $-11,17$ & 1 & 3,95 & 62,82 & 1 & yes \\
\hline & 7,02 & $-2,15$ & $-6,16$ & 1 & 2,42 & 19,00 & 1 & $-2,67$ & $-11,17$ & 1 & 3,95 & 62,82 & 1 & yes \\
\hline Panel F: Savings & ount ra & & & & & & & & & & & & & \\
\hline Czech Republic & 9 & $-3,00$ & $-10,53$ & 0 & 9,66 & 55,54 & 0 & $-3,67$ & $-11,82$ & 0 & 6,75 & 69,84 & 0 & no \\
\hline Lithuania & 9 & $-2,30$ & $-10,46$ & 1 & 2,94 & 54,70 & 1 & $-3,81$ & $-8,13$ & 0 & 10,23 & 33,53 & 0 & yes \\
\hline Slovak Republic & 9 & $-0,72$ & $-9,31$ & 1 & 1,31 & 43,47 & 1 & $-3,45$ & $-9,85$ & 0 & 6,20 & 48,58 & 1 & yes \\
\hline Slovenia & 9 & $-8,72$ & $-13,56$ & 0 & 42,27 & 92,12 & 0 & $-5,14$ & $-15,27$ & 0 & 14,54 & 117,08 & 0 & yes \\
\hline Panel G: Time de & it rates & & & & & & & & & & & & & \\
\hline Czech Republic & 8,01 & $-1,97$ & $-9,37$ & 1 & 2,41 & 43,94 & 1 & $-3,67$ & $-11,82$ & 0 & 6,75 & 69,84 & 0 & yes \\
\hline & 8,02 & $-1,87$ & $-7,16$ & 1 & 1,92 & 25,64 & 1 & $-3,67$ & $-11,82$ & 0 & 6,75 & 69,84 & 0 & yes \\
\hline & 8,03 & $-0,55$ & $-10,50$ & 1 & 4,34 & 55,15 & 1 & $-3,67$ & $-11,82$ & 0 & 6,75 & 69,84 & 0 & yes \\
\hline & 8,04 & $-1,96$ & $-8,75$ & 1 & 2,09 & 38,27 & 1 & $-3,67$ & $-11,82$ & 0 & 6,75 & 69,84 & 0 & yes \\
\hline Estonia & 8,01 & $-3,60$ & $-8,41$ & 0 & 6,99 & 35,38 & 0 & $-4,10$ & $-4,53$ & 0 & 8,46 & 10,25 & 0 & no \\
\hline & 8,02 & $-2,75$ & $-7,00$ & 1 & 3,94 & 24,72 & 1 & $-4,10$ & $-4,53$ & 0 & 8,46 & 10,25 & 0 & yes \\
\hline & 8,03 & $-3,69$ & $-7,35$ & 0 & 7,21 & 27,05 & 0 & $-4,10$ & $-4,53$ & 0 & 8,46 & 10,25 & 0 & no \\
\hline & 8,04 & $-2,74$ & $-4,75$ & 1 & 3,86 & 11,29 & 1 & $-4,10$ & $-4,53$ & 0 & 8,46 & 10,25 & 0 & yes \\
\hline & 8,05 & $-2,09$ & $-7,67$ & 1 & 2,27 & 29,39 & 1 & $-4,10$ & $-4,53$ & 0 & 8,46 & 10,25 & 0 & yes \\
\hline & 8,06 & $-5,20$ & $-11,62$ & 0 & 13,61 & 67,55 & 0 & $-4,10$ & $-4,53$ & 0 & 8,46 & 10,25 & 0 & no \\
\hline Hungary & 8,01 & 0,60 & $-4,51$ & 1 & 1,41 & 10,86 & 1 & $-2,31$ & $-7,95$ & 1 & 3,25 & 31,72 & 1 & yes \\
\hline & 8,02 & $-0,37$ & $-6,96$ & 1 & 1,34 & 24,78 & 1 & $-2,31$ & $-7,95$ & 1 & 3,25 & 31,72 & 1 & yes \\
\hline Latvia & 8,01 & $-3,77$ & $-9,12$ & 0 & 7,11 & 41,61 & 0 & $-2,78$ & $-15,47$ & 1 & 5,48 & 119,65 & 1 & yes \\
\hline & 8,02 & $-2,69$ & $-5,02$ & 1 & 3,61 & 12,60 & 1 & $-2,78$ & $-15,47$ & 1 & 5,48 & 119,65 & 1 & yes \\
\hline & 8,03 & $-2,80$ & $-7,76$ & 1 & 3,93 & 30,15 & 1 & $-2,78$ & $-15,47$ & 1 & 5,48 & 119,65 & 1 & yes \\
\hline & 8,04 & $-3,31$ & $-9,09$ & 0 & 5,54 & 41,33 & 1 & $-2,78$ & $-15,47$ & 1 & 5,48 & 119,65 & 1 & yes \\
\hline & 8,05 & $-3,16$ & $-8,33$ & 0 & 5,01 & 34,72 & 1 & $-2,78$ & $-15,47$ & 1 & 5,48 & 119,65 & 1 & yes \\
\hline & 8,06 & $-2,72$ & $-7,83$ & 1 & 3,94 & 30,64 & 1 & $-2,78$ & $-15,47$ & 1 & 5,48 & 119,65 & 1 & yes \\
\hline & 8,07 & $-3,75$ & $-9,10$ & 0 & 7,03 & 41,37 & 0 & $-2,78$ & $-15,47$ & 1 & 5,48 & 119,65 & 1 & yes \\
\hline & 8,08 & $-2,85$ & $-5,25$ & 1 & 4,07 & 13,79 & 1 & $-2,78$ & $-15,47$ & 1 & 5,48 & 119,65 & 1 & yes \\
\hline & 8,01 & $-3,18$ & $-7,76$ & 0 & 5,04 & 30,15 & 1 & $-2,78$ & $-15,47$ & 1 & 5,48 & 119,65 & 1 & yes \\
\hline & 8,10 & $-5,33$ & $-9,57$ & 0 & 14,21 & 45,82 & 0 & $-2,78$ & $-15,47$ & 1 & 5,48 & 119,65 & 1 & yes \\
\hline & 8,11 & $-3,35$ & $-8,49$ & 0 & 5,62 & 36,07 & 1 & $-2,78$ & $-15,47$ & 1 & 5,48 & 119,65 & 1 & yes \\
\hline & 8,12 & $-2,48$ & $-8,51$ & 1 & 3,36 & 36,28 & 1 & $-2,78$ & $-15,47$ & 1 & 5,48 & 119,65 & 1 & yes \\
\hline & 8,13 & $-2,97$ & $-6,95$ & 0 & 4,43 & 24,24 & 1 & $-2,78$ & $-15,47$ & 1 & 5,48 & 119,65 & 1 & yes \\
\hline & 8,14 & $-2,21$ & $-5,19$ & 1 & 2,60 & 13,53 & 1 & $-2,78$ & $-15,47$ & 1 & 5,48 & 119,65 & 1 & yes \\
\hline & 8,15 & $-2,33$ & $-4,25$ & 1 & 2,83 & 9,05 & 1 & $-2,78$ & $-15,47$ & 1 & 5,48 & 119,65 & 1 & yes \\
\hline & 8,16 & $-2,51$ & $-5,50$ & 1 & 3,42 & 15,12 & 1 & $-2,78$ & $-15,47$ & 1 & 5,48 & 119,65 & 1 & yes \\
\hline & 8,17 & $-2,86$ & $-4,82$ & 1 & 4,27 & 11,65 & 1 & $-2,78$ & $-15,47$ & 1 & 5,48 & 119,65 & 1 & yes \\
\hline & 8,18 & $-3,09$ & $-6,80$ & 0 & 5,15 & 23,13 & 1 & $-2,78$ & $-15,47$ & 1 & 5,48 & 119,65 & 1 & yes \\
\hline
\end{tabular}


Table B1: Full-period unit root tests

\begin{tabular}{|c|c|c|c|c|c|c|c|c|c|c|c|c|c|c|}
\hline \multirow{3}{*}{$\frac{\text { country }}{\text { Lithuania }}$} & \multirow[b]{2}{*}{ bankrate } & \multicolumn{6}{|c|}{ bank rate } & \multicolumn{6}{|c|}{ monetary policy rate } & \multirow{2}{*}{$\begin{array}{r}\text { Can cointe- } \\
\text { gration be } \\
\text { applied? }^{1}\end{array}$} \\
\hline & & \multirow{2}{*}{$\frac{\mathrm{t}(\text { level })}{-2,07}$} & \multicolumn{2}{|c|}{$\mathrm{t}($ diff) $\mathrm{I}(?)$ at $5 \%$} & \multirow{2}{*}{$\begin{array}{c}\mathrm{F} \text { (level) } \\
2,73\end{array}$} & \multicolumn{2}{|c|}{$\mathrm{F}(\mathrm{diff}) \mathrm{I}(?)$ at $5 \%$} & \multirow{2}{*}{$\frac{\mathrm{t}(\text { level })}{-3,81}$} & \multicolumn{2}{|c|}{$\mathrm{t}($ diff) $\mathrm{I}(?)$ at $5 \%$} & \multirow{2}{*}{$\frac{F(\text { level })}{10,23}$} & \multicolumn{2}{|c|}{$\mathrm{F}($ diff) $\mathrm{I}(?)$ at $5 \%$} & \\
\hline & 8,01 & & $-12,67$ & 1 & & 80,29 & 1 & & $-8,13$ & 0 & & 33,53 & 0 & yes \\
\hline & 8,02 & $-1,63$ & $-6,21$ & 1 & 1,34 & 19,31 & 1 & $-3,81$ & $-8,13$ & 0 & 10,23 & 33,53 & 0 & yes \\
\hline & 8,03 & $-2,27$ & $-9,62$ & 1 & 5,03 & 46,31 & 1 & $-3,81$ & $-8,13$ & 0 & 10,23 & 33,53 & 0 & yes \\
\hline & 8,04 & $-1,94$ & $-3,75$ & 1 & 1,91 & 7,07 & 1 & $-3,81$ & $-8,13$ & 0 & 10,23 & 33,53 & 0 & yes \\
\hline & 8,05 & $-3,31$ & $-10,31$ & 0 & 6,36 & 53,31 & 1 & $-3,81$ & $-8,13$ & 0 & 10,23 & 33,53 & 0 & yes \\
\hline & 8,06 & $-2,13$ & $-3,53$ & 1 & 2,46 & 6,32 & 2 & $-3,81$ & $-8,13$ & 0 & 10,23 & 33,53 & 0 & yes \\
\hline & 8,07 & $-1,91$ & $-9,22$ & 1 & 2,38 & 42,56 & 1 & $-3,81$ & $-8,13$ & 0 & 10,23 & 33,53 & 0 & yes \\
\hline & 8,08 & $-1,73$ & $-4,82$ & 1 & 1,51 & 11,76 & 1 & $-3,81$ & $-8,13$ & 0 & 10,23 & 33,53 & 0 & yes \\
\hline & 8,09 & $-3,02$ & $-9,07$ & 0 & 5,77 & 41,19 & 1 & $-3,81$ & $-8,13$ & 0 & 10,23 & 33,53 & 0 & yes \\
\hline & 8,10 & $-2,89$ & $-4,40$ & 0 & 5,66 & 9,69 & 1 & $-3,81$ & $-8,13$ & 0 & 10,23 & 33,53 & 0 & yes \\
\hline & 8,11 & $-4,34$ & $-8,91$ & 0 & 9,54 & 40,11 & 0 & $-3,81$ & $-8,13$ & 0 & 10,23 & 33,53 & 0 & no \\
\hline & 8,12 & $-2,18$ & $-4,94$ & 1 & 2,78 & 12,59 & 1 & $-3,81$ & $-8,13$ & 0 & 10,23 & 33,53 & 0 & yes \\
\hline & 8,13 & $-1,99$ & $-9,76$ & 1 & 3,39 & 47,68 & 1 & $-3,81$ & $-8,13$ & 0 & 10,23 & 33,53 & 0 & yes \\
\hline & 8,14 & $-1,87$ & $-3,61$ & 1 & 1,82 & 6,62 & 1 & $-3,81$ & $-8,13$ & 0 & 10,23 & 33,53 & 0 & yes \\
\hline & 8,15 & $-1,81$ & $-15,74$ & 1 & 2,05 & 126,11 & 1 & $-3,81$ & $-8,13$ & 0 & 10,23 & 33,53 & 0 & yes \\
\hline \multirow[t]{12}{*}{ Poland } & 8,01 & $-2,00$ & $-3,18$ & 1 & 2,14 & 5,11 & 2 & $-2,67$ & $-11,17$ & 1 & 3,95 & 62,82 & 1 & yes \\
\hline & 8,02 & $-2,06$ & $-3,80$ & 1 & 2,31 & 7,26 & 1 & $-2,67$ & $-11,17$ & 1 & 3,95 & 62,82 & 1 & yes \\
\hline & 8,03 & $-1,99$ & $-4,10$ & 1 & 2,08 & 8,41 & 1 & $-2,67$ & $-11,17$ & 1 & 3,95 & 62,82 & 1 & yes \\
\hline & 8,04 & $-2,05$ & $-4,11$ & 1 & 2,16 & 8,47 & 1 & $-2,67$ & $-11,17$ & 1 & 3,95 & 62,82 & 1 & yes \\
\hline & 8,05 & $-1,62$ & $-3,23$ & 1 & 1,37 & 5,25 & 2 & $-2,67$ & $-11,17$ & 1 & 3,95 & 62,82 & 1 & yes \\
\hline & 8,06 & $-1,50$ & $-3,35$ & 1 & 1,52 & 5,65 & 2 & $-2,67$ & $-11,17$ & 1 & 3,95 & 62,82 & 1 & yes \\
\hline & 8,07 & $-1,86$ & $-3,09$ & 1 & 1,86 & 4,81 & 2 & $-2,67$ & $-11,17$ & 1 & 3,95 & 62,82 & 1 & yes \\
\hline & 8,08 & $-1,87$ & $-3,60$ & 1 & 1,94 & 6,50 & 1 & $-2,67$ & $-11,17$ & 1 & 3,95 & 62,82 & 1 & yes \\
\hline & 8,09 & $-1,89$ & $-3,58$ & 1 & 1,94 & 6,43 & 2 & $-2,67$ & $-11,17$ & 1 & 3,95 & 62,82 & 1 & yes \\
\hline & 8,10 & $-1,99$ & $-3,80$ & 1 & 2,05 & 7,27 & 1 & $-2,67$ & $-11,17$ & 1 & 3,95 & 62,82 & 1 & yes \\
\hline & 8,11 & $-1,70$ & $-4,26$ & 1 & 1,54 & 9,08 & 1 & $-2,67$ & $-11,17$ & 1 & 3,95 & 62,82 & 1 & yes \\
\hline & 8,12 & $-1,16$ & $-3,36$ & 1 & 0,88 & 5,65 & 2 & $-2,67$ & $-11,17$ & 1 & 3,95 & 62,82 & 1 & yes \\
\hline \multirow[t]{7}{*}{ Slovak Republic } & 8,01 & $-1,30$ & $-6,48$ & 1 & 1,46 & 21,01 & 1 & $-3,45$ & $-9,85$ & 0 & 6,20 & 48,58 & 1 & yes \\
\hline & 8,02 & $-1,16$ & $-5,32$ & 1 & 1,53 & 14,19 & 1 & $-3,45$ & $-9,85$ & 0 & 6,20 & 48,58 & 1 & yes \\
\hline & 8,03 & $-1,74$ & $-6,56$ & 1 & 2,89 & 21,59 & 1 & $-3,45$ & $-9,85$ & 0 & 6,20 & 48,58 & 1 & yes \\
\hline & 8,04 & $-1,26$ & $-6,73$ & 1 & 0,93 & 22,67 & 1 & $-3,45$ & $-9,85$ & 0 & 6,20 & 48,58 & 1 & yes \\
\hline & 8,05 & $-2,72$ & $-8,29$ & 1 & 3,80 & 34,35 & 1 & $-3,45$ & $-9,85$ & 0 & 6,20 & 48,58 & 1 & yes \\
\hline & 8,06 & $-2,31$ & $-6,85$ & 1 & 2,68 & 23,46 & 1 & $-3,45$ & $-9,85$ & 0 & 6,20 & 48,58 & 1 & yes \\
\hline & 8,07 & $-2,03$ & $-7,51$ & 1 & 2,22 & 28,23 & 1 & $-3,45$ & $-9,85$ & 0 & 6,20 & 48,58 & 1 & yes \\
\hline \multirow[t]{5}{*}{ Slovenia } & 8,01 & $-8,72$ & $-14,15$ & 0 & 42,03 & 100,29 & 0 & $-5,14$ & $-15,27$ & 0 & 14,54 & 117,08 & 0 & no \\
\hline & 8,02 & $-8,70$ & $-14,12$ & 0 & 41,74 & 99,85 & 0 & $-5,14$ & $-15,27$ & 0 & 14,54 & 117,08 & 0 & no \\
\hline & 8,03 & $-8,61$ & $-13,96$ & 0 & 40,92 & 97,61 & 0 & $-5,14$ & $-15,27$ & 0 & 14,54 & 117,08 & 0 & no \\
\hline & 8,04 & $-8,65$ & $-13,98$ & 0 & 41,33 & 97,96 & 0 & $-5,14$ & $-15,27$ & 0 & 14,54 & 117,08 & 0 & no \\
\hline & 8,05 & $-8,69$ & $-14,02$ & 0 & 41,59 & 98,53 & 0 & $-5,14$ & $-15,27$ & 0 & 14,54 & 117,08 & 0 & no \\
\hline
\end{tabular}

The critical values for the unit root test at $5 \%$ are -2.88 for the t-test and 6.49 for the F-test. ${ }^{1}$ No if both rates are $\mathrm{I}(0)$. 
Table B2: Full-period symmetric cointegration of bank rate and money market rate

\begin{tabular}{|c|c|c|c|c|c|c|c|c|c|c|}
\hline \multirow[b]{2}{*}{ country } & \multirow[b]{2}{*}{ rate } & \multirow{2}{*}{$\begin{array}{c}\text { obser- } \\
\text { vations }\end{array}$} & \multicolumn{2}{|c|}{ intercept } & \multicolumn{2}{|c|}{ slope } & \multirow[b]{2}{*}{ DW } & \multirow[b]{2}{*}{ DF } & \multirow[b]{2}{*}{$\mathrm{ADF}$} & \multirow{2}{*}{$\begin{array}{r}\text { cointe- } \\
\text { gration? }\end{array}$} \\
\hline & & & coefficient & t-statistic & coefficient & t-statistic & & & & \\
\hline \multicolumn{11}{|c|}{ Panel A: Short-term corporate loan rates } \\
\hline Czech Republic & 4 & 36 & 1,17 & 21,79 & 0,95 & 69,05 & 0,96 & $-3,24$ & & yes \\
\hline Estonia & 4 & 81 & 5,93 & 15,34 & 0,77 & 14,55 & 0,52 & $-3,54$ & & yes \\
\hline Hungary & 4 & 108 & $-0,30$ & $-1,03$ & 1,10 & 67,17 & 0,16 & $-2,87$ & & 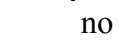 \\
\hline Latvia & 4 & 60 & 4,12 & 3,59 & 1,43 & 5,37 & 0,37 & $-2,58$ & & no \\
\hline Lithuania & 4 & 60 & 6,03 & 9,59 & 1,13 & 7,18 & 0,60 & $-3,46$ & & yes \\
\hline Poland & 4 & 79 & 2,79 & 5,42 & 0,99 & 32,49 & 1,35 & $-6,24$ & $-3,38$ & yes \\
\hline Slovak Republic & 4 & 98 & 7,22 & 10,57 & 0,65 & 12,22 & 0,24 & $-2,81$ & $-2,40$ & no \\
\hline \multicolumn{11}{|c|}{ Panel B: Long-term corporate loan rates } \\
\hline Estonia & 5 & 81 & 6,24 & 18,90 & 0,58 & 12,70 & 0,86 & $-4,76$ & & yes \\
\hline Hungary & 5 & 108 & 0,20 & 0,76 & 1,11 & 74,53 & 0,69 & $-5,20$ & & yes \\
\hline Latvia & 5 & 60 & 5,51 & 7,63 & 1,05 & 6,25 & 0,55 & $-3,70$ & $-2,95$ & yes \\
\hline \multirow[t]{2}{*}{ Lithuania } & 5,01 & 60 & 5,91 & 10,75 & 1,00 & 7,25 & 0,74 & $-4,26$ & & yes \\
\hline & 5,02 & 60 & 5,95 & 10,84 & 0,83 & 6,05 & 0,56 & $-3,04$ & $-2,42$ & yes \\
\hline \multirow[t]{2}{*}{ Poland } & 5,01 & 79 & 3,50 & 6,56 & 0,97 & 30,66 & 1,21 & $-5,76$ & $-3,22$ & yes \\
\hline & 5,02 & 66 & 4,44 & 8,89 & 0,90 & 28,11 & 1,12 & $-5,45$ & $-2,90$ & yes \\
\hline \multicolumn{11}{|c|}{ Panel C: Consumer loan rates } \\
\hline Czech Republic & 3 & 132 & 8,91 & 32,25 & 0,26 & 9,69 & 0,71 & $-5,49$ & $-1,63$ & yes \\
\hline Estonia & 3 & 81 & 11,39 & 29,58 & 0,21 & 3,89 & 0,88 & $-4,87$ & $-5,29$ & yes \\
\hline Hungary & 3 & 108 & 16,62 & 61,99 & 0,51 & 34,19 & 0,29 & $-2,85$ & & no \\
\hline \multirow[t]{2}{*}{ Latvia } & 3,01 & 60 & 11,25 & 15,31 & 0,82 & 4,82 & 0,71 & $-3,46$ & & yes \\
\hline & 3,02 & 60 & 1,93 & 0,96 & 2,76 & 5,88 & 0,41 & $-2,60$ & $-0,99$ & yes \\
\hline \multirow[t]{2}{*}{ Lithuania } & 3,01 & 60 & 9,26 & 15,70 & 0,82 & 5,53 & 0,92 & $-4,34$ & & yes \\
\hline & 3,02 & 60 & 9,59 & 22,56 & 0,77 & 7,24 & 0,56 & $-3,19$ & & yes \\
\hline Poland & 3 & 79 & 12,53 & 25,24 & 0,60 & 20,39 & 0,58 & $-3,64$ & $-2,15$ & yes \\
\hline Slovak Republic & 3 & 98 & 10,05 & 21,70 & 0,02 & 0,42 & 0,35 & $-3,93$ & & yes \\
\hline Panel D: Mortgag & rates & & & & & & & & & \\
\hline Estonia & 2 & 81 & 8,21 & 17,86 & 0,40 & 6,33 & 0,16 & $-1,45$ & $-0,85$ & no \\
\hline Hungary & 2 & 108 & 6,31 & 11,52 & 0,98 & 31,90 & 0,15 & $-1,23$ & & no \\
\hline Latvia & 2 & 60 & 7,55 & 8,43 & 1,01 & 4,88 & 0,34 & $-2,31$ & $-1,95$ & no \\
\hline Lithuania & 2,01 & 60 & 5,73 & 7,84 & 1,24 & 6,77 & 0,58 & $-3,47$ & & yes \\
\hline & 2,02 & 60 & 6,51 & 14,01 & 0,06 & 0,55 & 0,34 & $-2,27$ & $-1,27$ & no \\
\hline Panel E: Current & ccount & posit rate & & & & & & & & \\
\hline Hungary & 7 & 108 & $-0,38$ & $-1,74$ & 0,36 & 29,60 & 0,12 & $-1,11$ & $-1,59$ & no \\
\hline Poland & 7,01 & 79 & $-3,93$ & $-6,39$ & 0,70 & 19,32 & 0,43 & $-3,21$ & $-2,88$ & yes \\
\hline & 7,02 & 79 & $-2,32$ & $-5,40$ & 0,45 & 17,43 & 0,37 & $-2,79$ & $-1,71$ & no \\
\hline Panel F: Savings & $\overline{\operatorname{ccount}}$ & & & & & & & & & \\
\hline Czech Republic & 9 & 132 & 1,30 & 17,76 & 0,07 & 10,06 & 0,18 & $-2,13$ & $-1,39$ & no \\
\hline Lithuania & 9 & 60 & 3,00 & 6,88 & 0,56 & 5,14 & 0,71 & $-3,47$ & $-1,97$ & yes \\
\hline Slovak Republic & 9 & 98 & 2,02 & 13,75 & 0,08 & 7,11 & 0,11 & $-1,45$ & $-0,73$ & no \\
\hline Panel G: Time de & osit ra & & & & & & & & & \\
\hline Czech Republic & 8,01 & 132 & 1,62 & 5,86 & 0,62 & 22,88 & 0,35 & $-3,46$ & $-2,59$ & yes \\
\hline & 8,02 & 132 & 2,19 & 5,21 & 0,62 & 15,28 & 0,27 & $-3,14$ & $-1,70$ & no \\
\hline & 8,03 & 132 & 3,91 & 6,25 & 0,28 & 4,65 & 0,04 & $-1,96$ & $-1,66$ & no \\
\hline & 8,04 & 132 & 2,10 & 6,11 & 0,58 & 17,40 & 0,23 & $-2,89$ & $-1,80$ & no \\
\hline Estonia & 8,01 & 96 & 1,43 & 5,95 & 0,48 & 14,06 & 0,62 & $-4,33$ & & yes \\
\hline & 8,02 & 96 & 2,24 & 6,17 & 0,60 & 11,72 & 0,11 & $-2,06$ & $-2,47$ & no \\
\hline & 8,03 & 96 & 0,84 & 3,44 & 0,57 & 16,45 & 0,45 & $-3,46$ & $-3,72$ & yes \\
\hline & 8,04 & 81 & 1,41 & 5,81 & 0,67 & 19,98 & 0,30 & $-2,73$ & $-4,65$ & yes \\
\hline & 8,05 & 96 & 2,42 & 7,05 & 0,72 & 14,89 & 0,21 & $-2,56$ & $-2,85$ & no \\
\hline & 8,06 & 96 & 4,15 & 9,19 & 0,50 & 7,79 & 0,52 & $-3,86$ & & yes \\
\hline Hungary & 8,01 & 108 & $-0,47$ & $-3,41$ & 0,82 & 107,64 & 0,77 & $-5,04$ & $-5,89$ & yes \\
\hline & 8,02 & 108 & $-1,23$ & $-6,11$ & 0,90 & 79,97 & 0,57 & $-3,85$ & & yes \\
\hline
\end{tabular}


Table B2: Full-period symmetric cointegration of bank rate and money market rate

\begin{tabular}{|c|c|c|c|c|c|c|c|c|c|c|}
\hline \multirow[b]{2}{*}{ country } & \multirow[b]{2}{*}{ rate } & \multirow{2}{*}{$\begin{array}{c}\text { obser- } \\
\text { vations }\end{array}$} & \multicolumn{2}{|c|}{ intercept } & \multicolumn{2}{|c|}{ slope } & \multirow[b]{2}{*}{ DW } & \multirow[b]{2}{*}{ DF } & \multirow[b]{2}{*}{$\mathrm{ADF}$} & \multirow{2}{*}{$\begin{array}{r}\text { cointe- } \\
\text { gration? }\end{array}$} \\
\hline & & & coefficient & t-statistic & coefficient & t-statistic & & & & \\
\hline \multirow[t]{18}{*}{ Latvia } & 8,01 & 60 & 0,45 & 2,83 & 0,77 & 21,04 & 1,69 & $-6,56$ & $-6,25$ & yes \\
\hline & 8,02 & 60 & 2,06 & 9,01 & 0,56 & 10,56 & 0,75 & $-3,61$ & $-1,72$ & yes \\
\hline & 8,03 & 60 & 2,89 & 8,37 & 0,54 & 6,68 & 0,73 & $-3,53$ & $-0,61$ & yes \\
\hline & 8,04 & 60 & 4,32 & 12,11 & 0,38 & 4,56 & 0,44 & $-2,53$ & $-1,69$ & yes \\
\hline & 8,05 & 60 & 1,39 & 6,38 & 0,68 & 13,46 & 0,85 & $-3,97$ & $-2,15$ & yes \\
\hline & 8,06 & 60 & 5,01 & 12,15 & 0,30 & 3,17 & 0,47 & $-2,63$ & $-1,77$ & yes \\
\hline & 8,07 & 60 & 0,42 & 2,65 & 0,78 & 20,95 & 1,67 & $-6,50$ & & yes \\
\hline & 8,08 & 60 & 1,73 & 7,15 & 0,64 & 11,28 & 0,88 & $-3,99$ & $-1,24$ & yes \\
\hline & 8,09 & 60 & 2,46 & 6,71 & 0,64 & 7,54 & 1,00 & $-4,43$ & $-0,75$ & yes \\
\hline & 8,10 & 60 & 4,08 & 8,22 & 0,34 & 2,97 & 1,09 & $-4,62$ & $-3,21$ & yes \\
\hline & 8,11 & 60 & 1,01 & 4,94 & 0,73 & 15,37 & 1,09 & $-4,66$ & & yes \\
\hline & 8,12 & 60 & 5,52 & 10,24 & 0,07 & 0,59 & 0,85 & $-3,96$ & $-2,23$ & yes \\
\hline & 8,13 & 60 & 1,68 & 7,73 & 0,47 & 9,32 & 0,59 & $-3,10$ & $-3,53$ & yes \\
\hline & 8,14 & 60 & 3,13 & 13,58 & 0,32 & 5,90 & 0,48 & $-2,70$ & $-1,91$ & yes \\
\hline & 8,15 & 60 & 3,44 & 11,28 & 0,40 & 5,59 & 0,33 & $-2,09$ & $-0,83$ & no \\
\hline & 8,16 & 60 & 4,55 & 13,70 & 0,35 & 4,55 & 0,25 & $-1,73$ & $-0,73$ & no \\
\hline & 8,17 & 60 & 3,66 & 11,58 & 0,38 & 5,21 & 0,27 & $-1,89$ & $-0,84$ & no \\
\hline & 8,18 & 60 & 4,61 & 11,03 & 0,46 & 4,71 & 0,34 & $-2,13$ & $-1,42$ & no \\
\hline \multirow[t]{15}{*}{ Lithuania } & 8,01 & 121 & $-1,00$ & $-2,87$ & 0,81 & 64,41 & 0,98 & $-6,54$ & $-7,75$ & yes \\
\hline & 8,02 & 60 & 1,56 & 4,04 & 0,70 & 7,19 & 0,42 & $-2,72$ & & yes \\
\hline & 8,03 & 121 & 2,22 & 7,54 & 0,65 & 60,87 & 0,76 & $-6,69$ & & yes \\
\hline & 8,04 & 60 & 2,13 & 5,08 & 0,72 & 6,88 & 0,45 & $-2,81$ & & yes \\
\hline & 8,05 & 121 & 2,54 & 6,99 & 0,70 & 52,76 & 1,43 & $-8,36$ & $-8,29$ & yes \\
\hline & 8,06 & 60 & 2,63 & 6,14 & 0,74 & 6,87 & 0,43 & $-2,71$ & & yes \\
\hline & 8,07 & 121 & 2,86 & 6,89 & 0,70 & 46,58 & 1,26 & $-8,67$ & $-6,41$ & yes \\
\hline & 8,08 & 60 & 3,17 & 7,27 & 0,75 & 6,88 & 0,43 & $-2,71$ & & yes \\
\hline & 8,09 & 121 & 4,33 & 8,77 & 0,52 & 29,07 & 1,35 & $-7,90$ & $-5,82$ & yes \\
\hline & 8,10 & 60 & 4,04 & 8,88 & 0,69 & 6,03 & 0,32 & $-2,15$ & & no \\
\hline & 8,11 & 84 & 4,34 & 11,50 & 0,47 & 6,91 & 0,72 & $-4,94$ & $-2,88$ & yes \\
\hline & 8,12 & 60 & 3,75 & 8,06 & 0,69 & 5,88 & 0,35 & $-2,34$ & & no \\
\hline & 8,13 & 121 & 0,81 & 2,99 & 0,70 & 70,33 & 1,00 & $-7,75$ & $-7,33$ & yes \\
\hline & 8,14 & 60 & 2,57 & 6,16 & 0,72 & 6,86 & 0,41 & $-2,64$ & & yes \\
\hline & 8,15 & 121 & 0,81 & 6,47 & 0,19 & 42,20 & 0,97 & $-6,15$ & $-4,28$ & yes \\
\hline \multirow[t]{12}{*}{ Poland } & 8,01 & 79 & $-1,36$ & $-3,39$ & 0,82 & 34,57 & 1,35 & $-6,25$ & $-3,33$ & yes \\
\hline & 8,02 & 79 & $-1,75$ & $-4,07$ & 0,85 & 33,27 & 1,29 & $-6,06$ & $-3,25$ & yes \\
\hline & 8,03 & 79 & $-1,77$ & $-3,87$ & 0,87 & 32,21 & 1,20 & $-5,80$ & $-3,26$ & yes \\
\hline & 8,04 & 79 & $-1,68$ & $-3,37$ & 0,91 & 30,74 & 1,08 & $-5,41$ & $-3,16$ & yes \\
\hline & 8,05 & 79 & $-1,11$ & $-2,21$ & 0,91 & 30,30 & 1,12 & $-5,57$ & $-3,23$ & yes \\
\hline & 8,06 & 63 & 0,20 & 0,25 & 0,84 & 19,37 & 1,26 & $-5,36$ & $-2,88$ & yes \\
\hline & 8,07 & 79 & $-1,23$ & $-3,32$ & 0,82 & 37,47 & 1,48 & $-6,73$ & & yes \\
\hline & 8,08 & 79 & $-1,38$ & $-3,35$ & 0,86 & 34,94 & 1,38 & $-6,38$ & $-3,57$ & yes \\
\hline & 8,09 & 79 & $-1,55$ & $-3,46$ & 0,88 & 33,30 & 1,29 & $-6,09$ & $-3,46$ & yes \\
\hline & 8,10 & 79 & $-1,78$ & $-3,57$ & 0,93 & 31,47 & 1,11 & $-5,50$ & $-3,23$ & yes \\
\hline & 8,11 & 79 & $-0,71$ & $-1,44$ & 0,88 & 29,94 & 1,11 & $-5,52$ & $-3,28$ & yes \\
\hline & 8,12 & 63 & 0,34 & 0,41 & 0,83 & 18,40 & 1,22 & $-5,33$ & $-3,17$ & yes \\
\hline \multirow[t]{7}{*}{ Slovak Republic } & 8,01 & 98 & 1,14 & 1,95 & 0,71 & 15,51 & 0,25 & $-2,40$ & $-1,26$ & no \\
\hline & 8,02 & 98 & 2,20 & 2,89 & 0,65 & 11,02 & 0,20 & $-2,46$ & $-1,53$ & no \\
\hline & 8,03 & 98 & 3,82 & 5,14 & 0,50 & 8,62 & 0,14 & $-2,02$ & $-2,15$ & no \\
\hline & 8,04 & 98 & 5,70 & 10,19 & 0,28 & 6,36 & 0,09 & $-2,21$ & $-1,48$ & no \\
\hline & 8,05 & 98 & 6,14 & 10,47 & 0,24 & 5,16 & 0,12 & $-3,10$ & $-3,51$ & yes \\
\hline & 8,06 & 98 & 8,95 & 11,27 & 0,20 & 3,25 & 0,07 & $-2,08$ & $-2,35$ & no \\
\hline & 8,07 & 98 & 5,66 & 15,71 & $-0,06$ & $-2,23$ & 0,14 & $-1,30$ & $-2,54$ & no \\
\hline
\end{tabular}

Note: Cointegration is considered to exist if at least two test statistics are significant at $10 \%$ level or if at least one test-statistic is significant at $5 \%$ level. No results for Slovenia are reported because these rates were found to be I(0) in table B1. 


\begin{tabular}{|c|c|c|c|c|c|c|c|c|c|c|c|c|c|c|c|}
\hline country & rate & $\mathrm{TAR}^{0}$ & TAR* & BTAR* & MTAR $^{0}$ & MTAR* & AIC & model & $\mathrm{H}_{0}: \Sigma_{\mathrm{j}} \rho_{\mathrm{j}}=0$ & $\mathrm{H}_{0}: \rho_{1}=\rho_{2}$ & $\mathrm{H}_{0}: \rho_{1}=\rho_{3}$ & $H_{0}: \rho_{2}=\rho_{3}$ & cointegration? & cointegration? & model \\
\hline \multicolumn{16}{|c|}{ Panel A: Short-term corporate loan rates } \\
\hline Czech Republic & 4 & $-42,95$ & $-43,54$ & $-46,68$ & $-44,21$ & $-47,19$ & $-47,19$ & MTAR* & 7,44 & 3,46 & & & no & yes & SYM \\
\hline Estonia & 4 & 368,19 & 366,62 & 360,27 & 367,26 & 364,06 & 360,27 & BTAR* & 7,12 & 9,62 & 0,00 & 9,62 & no & yes & SYM \\
\hline Hungary & 4 & 310,00 & 309,31 & 308,87 & 310,53 & 300,11 & 300,11 & MTAR* & 10,04 & 11,23 & & & yes,asym & no & MTAR* \\
\hline Latvia & 4 & 280,51 & 276,17 & 268,41 & 279,03 & 275,66 & 268,41 & BTAR* & 7,27 & 15,11 & 4,39 & 10,19 & no & no & STD \\
\hline Lithuania & 4 & 288,10 & 280,75 & 279,34 & 283,91 & 275,72 & 275,72 & MTAR* & 9,85 & 13,67 & & & yes,asym & yes & MTAR* \\
\hline Poland & 4 & 369,00 & 367,98 & 366,06 & 367,63 & 365,87 & 365,87 & MTAR* & 5,90 & 3,04 & & & no & yes & SYM \\
\hline Slovak Republic & 4 & 448,82 & 446,17 & 437,12 & 449,69 & 443,96 & 437,12 & BTAR* & 5,96 & 12,15 & 3,16 & 13,80 & no & no & STD \\
\hline \multicolumn{16}{|c|}{ Panel B: Long-term corporate loan rates } \\
\hline Estonia & 5 & 370,98 & 368,12 & 364,10 & 368,12 & 364,61 & 364,10 & BTAR* & 8,26 & 7,99 & 0,05 & 7,12 & no & yes & SYM \\
\hline Hungary & 5 & 433,01 & 432,01 & 425,17 & 432,92 & 429,47 & 425,17 & BTAR* & 7,19 & 9,35 & 0,06 & 8,98 & no & yes & SYM \\
\hline Latvia & 5 & 226,70 & 224,23 & 224,78 & 227,42 & 218,35 & 218,35 & MTAR* & 8,81 & 10,55 & & & no & yes & SYM \\
\hline \multirow{2}{*}{ Lithuania } & 5,01 & 278,38 & 277,59 & 275,47 & 278,05 & 275,08 & 275,08 & MTAR* & 4,15 & 3,10 & & & no & yes & SYM \\
\hline & 5,02 & 257,02 & 249,48 & 244,37 & 250,17 & 249,86 & 244,37 & BTAR* & 5,51 & 6,69 & 2,09 & 13,82 & no & yes & SYM \\
\hline \multirow[t]{2}{*}{ Poland } & 5,01 & 363,93 & 362,39 & 359,04 & 362,10 & 359,77 & 359,04 & BTAR* & 4,55 & 5,86 & 0,55 & 6,69 & no & yes & SYM \\
\hline & 5,02 & 259,70 & 256,47 & 258,05 & 259,34 & 255,37 & 255,37 & MTAR* & 5,15 & 6,94 & & & no & yes & SYM \\
\hline \multicolumn{16}{|c|}{ Panel C: Consumer loan rates } \\
\hline Czech Republic & 3 & 629,52 & 628,67 & 628,95 & 630,82 & 629,94 & 628,67 & TAR* & 2,91 & 3,11 & & & no & yes & SYM \\
\hline Estonia & 3 & 396,59 & 395,95 & 396,17 & 394,63 & 391,36 & 391,36 & MTAR* & 15,04 & 5,08 & & & yes,asym & yes & MTAR* \\
\hline Hungary & 3 & 361,37 & 355,03 & 348,64 & 361,39 & 357,58 & 348,64 & BTAR* & 7,02 & 11,67 & 0,17 & 8,15 & no & no & STD \\
\hline \multirow[t]{2}{*}{ Latvia } & 3,01 & 219,28 & 218,49 & 218,56 & 219,23 & 216,63 & 216,63 & MTAR* & 2,95 & 2,68 & & & no & yes & SYM \\
\hline & 3,02 & 343,16 & 337,36 & 339,36 & 341,75 & 338,55 & 337,36 & TAR* & 3,71 & 6,85 & & & no & yes & SYM \\
\hline \multirow[t]{2}{*}{ Lithuania } & 3,01 & 301,77 & 295,11 & 297,11 & 294,74 & 294,02 & 294,02 & MTAR* & 8,72 & 9,28 & & & no & yes & SYM \\
\hline & 3,02 & 243,44 & 239,66 & 230,06 & 242,67 & 235,33 & 230,06 & BTAR* & 7,04 & 0,08 & 13,15 & 14,26 & no & yes & SYM \\
\hline Poland & 3 & 308,31 & 307,66 & 306,62 & 308,05 & 303,67 & 303,67 & MTAR* & 5,10 & 4,42 & & & no & yes & SYM \\
\hline Slovak Republic & 3 & 351,09 & 350,36 & 349,15 & 352,83 & 346,95 & 346,95 & MTAR* & 8,13 & 5,67 & & & no & yes & SYM \\
\hline \multicolumn{16}{|c|}{ Panel D: Mortgage rates } \\
\hline Estonia & 2 & 310,90 & 308,98 & 308,34 & 314,89 & 309,31 & 308,34 & BTAR* & 3,43 & 4,38 & 5,65 & 2,84 & no & no & STD \\
\hline Hungary & 2 & 428,91 & 422,91 & 424,83 & 427,21 & 418,89 & 418,89 & MTAR* & 6,39 & 11,27 & & & no & no & STD \\
\hline Latvia & 2 & 241,42 & 238,66 & 236,14 & 238,67 & 225,76 & 225,76 & MTAR* & 11,10 & 17,56 & & & yes,asym & no & MTAR* \\
\hline \multirow[t]{2}{*}{ Lithuania } & 2,01 & 300,89 & 299,81 & 298,48 & 300,51 & 299,70 & 298,48 & BTAR* & 2,58 & 3,95 & 0,09 & 3,11 & no & yes & SYM \\
\hline & 2,02 & 212,92 & 212,48 & 202,73 & 212,64 & 207,37 & 202,73 & BTAR* & 4,46 & 11,89 & 0,07 & 11,92 & no & no & STD \\
\hline \multicolumn{16}{|c|}{ Panel E: Current account deposit rates } \\
\hline Hungary & 7 & 218,25 & 215,53 & 217,16 & 217,91 & 208,50 & 208,50 & MTAR* & 6,47 & 9,92 & & & no & no & STD \\
\hline \multirow[t]{2}{*}{$\begin{array}{l}\text { Poland } \\
\text { Pol }\end{array}$} & 7,01 & 315,98 & 309,16 & 303,38 & 309,96 & 301,85 & 301,85 & MTAR* & 9,13 & 15,09 & & & no & yes & SYM \\
\hline & 7,02 & 257,01 & 251,49 & 249,85 & 253,01 & 243,57 & 243,57 & MTAR* & 8,41 & 13,62 & & & no & no & STD \\
\hline \multicolumn{16}{|c|}{ Panel F: Savings account rates } \\
\hline Czech Republic & 9 & 173,07 & 172,55 & 165,46 & 172,66 & 167,88 & 165,46 & BTAR* & 3,55 & 9,43 & 0,05 & 9,00 & no & no & STD \\
\hline Lithuania & 9 & 251,26 & 246,93 & 242,52 & 252,10 & 240,62 & 240,62 & MTAR* & 7,47 & 12,07 & & & no & yes & SYM \\
\hline Slovak Republic & 9 & 112,45 & 109,85 & 108,04 & 113,78 & 106,66 & 106,66 & MTAR* & 3,84 & 7,16 & & & no & no & STD \\
\hline \multicolumn{16}{|c|}{ Panel G: Time deposit rates } \\
\hline \multirow[t]{4}{*}{ Czech Republic } & 8,01 & 574,61 & 566,59 & 558,82 & 574,15 & 556,25 & 556,25 & MTAR* & 15,86 & 26,55 & & & yes,asym & yes & MTAR* \\
\hline & 8,02 & 639,41 & 634,09 & 544,09 & 637,41 & 629,18 & 544,09 & BTAR* & 50,65 & 137,91 & 7,24 & 138,22 & yes,asym & no & BTAR* \\
\hline & 8,03 & 502,66 & 498,33 & 484,46 & 501,93 & 495,52 & 484,46 & BTAR* & 7,82 & 2,82 & 19,03 & 20,50 & no & no & STD \\
\hline & 8,04 & 573,11 & 562,42 & 554,78 & 569,15 & 553,19 & 553,19 & MTAR* & 14,39 & 25,82 & & & yes,asym & no & MTAR* \\
\hline Estonia & 8,01 & 385,58 & 381,98 & 381,00 & 385,91 & 380,31 & 380,31 & MTAR* & 8,86 & 5,55 & & & no & yes & SYM \\
\hline & 8,02 & 310,24 & 308,17 & 301,37 & 309,71 & 303,96 & 301,37 & BTAR* & 5,80 & 5,09 & 2,63 & 8,52 & no & no & STD \\
\hline & 8,03 & 365,85 & 365,17 & 364,34 & 365,39 & 363,50 & 363,50 & MTAR* & 7,27 & 2,33 & & & no & yes & SYM \\
\hline & 8,04 & 227,76 & 225,50 & 225,91 & 227,21 & 219,21 & 219,21 & MTAR* & 10,96 & 8,36 & & & yes,asym & yes & MTAR* \\
\hline & 8,05 & 356,66 & 354,46 & 353,65 & 356,86 & 339,57 & 339,57 & MTAR* & 13,10 & 17,78 & & & yes,asym & no & MTAR* \\
\hline & 8,06 & 477,46 & 474,01 & 471,60 & 476,84 & 402,85 & 402,85 & MTAR* & 59,16 & 108,70 & & & yes,asym & yes & MTAR* \\
\hline Hungary & 8,01 & 284,72 & 284,59 & 283,40 & 281,55 & 279,92 & 279,92 & MTAR* & 18,31 & 4,81 & & & yes,asym & yes & MTAR* \\
\hline & 8.02 & 373.93 & 3766 & 373.16 & 37462 & 371.44 & 371.44 & MTAR* & 6.50 & 321 & & & & ves & \\
\hline
\end{tabular}




\begin{tabular}{|c|c|c|c|c|c|c|c|c|c|c|c|c|c|c|c|}
\hline \multirow[b]{2}{*}{ country } & \multirow[b]{2}{*}{ rate } & \multicolumn{5}{|c|}{ AIC } & \multirow{2}{*}{$\begin{array}{r}\text { minimum } \\
\text { AIC }\end{array}$} & \multirow{2}{*}{$\begin{array}{r}\text { TAR-type } \\
\text { model }\end{array}$} & \multicolumn{5}{|c|}{ asymmetric cointegration testing } & \multirow{2}{*}{$\begin{array}{l}\text { Engle-GrangeI } \\
\text { cointegration? }\end{array}$} & \multirow{2}{*}{$\begin{array}{r}\text { Pass-through } \\
\text { model }\end{array}$} \\
\hline & & $\mathrm{TAR}^{0}$ & TAR* & BTAR* & MTAR $^{0}$ & MTAR* & & & $\mathrm{H}_{0}: \Sigma_{\mathrm{j}} \rho_{\mathrm{j}}=0$ & $H_{0}: \rho_{1}=\rho_{2}$ & $H_{0}: \rho_{1}=\rho_{3}$ & $\mathrm{H}_{0}: \rho_{2}=\rho_{3}$ & cointegration? & & \\
\hline \multirow[t]{18}{*}{ Latvia } & 8,01 & 116,65 & 116,07 & 116,18 & 116,12 & 115,23 & 115,23 & MTAR* & 7,84 & 1,43 & & & no & yes & SYM \\
\hline & 8,02 & 130,92 & 129,27 & 129,00 & 130,81 & 125,59 & 125,59 & MTAR* & 2,83 & 5,02 & & & no & yes & SYM \\
\hline & 8,03 & 166,06 & 164,55 & 161,68 & 165,72 & 159,55 & 159,55 & MTAR* & 3,44 & 6,45 & & & no & yes & SYM \\
\hline & 8,04 & 153,36 & 152,26 & 148,65 & 153,49 & 150,65 & 148,65 & BTAR* & 2,41 & 6,24 & 0,01 & 6,17 & no & yes & SYM \\
\hline & 8,05 & 137,32 & 136,72 & 134,91 & 135,19 & 133,60 & 133,60 & MTAR* & 3,19 & 3,52 & & & no & yes & SYM \\
\hline & 8,06 & 179,03 & 178,54 & 174,04 & 178,23 & 174,96 & 174,04 & BTAR* & 2,66 & 6,06 & 0,40 & 7,03 & no & yes & SYM \\
\hline & 8,07 & 118,48 & 117,94 & 118,92 & 118,07 & 117,10 & 117,10 & MTAR* & 7,68 & 1,40 & & & no & yes & SYM \\
\hline & 8,08 & 143,49 & 142,35 & 143,66 & 143,01 & 136,28 & 136,28 & MTAR* & 4,00 & 6,96 & & & no & yes & SYM \\
\hline & 8,01 & 181,64 & 180,85 & 177,04 & 181,76 & 180,67 & 177,04 & BTAR* & 2,37 & 6,11 & 0,42 & 6,12 & no & yes & SYM \\
\hline & 8,10 & 224,05 & 222,50 & 218,78 & 224,23 & 223,30 & 218,78 & BTAR* & 3,04 & 6,33 & 0,00 & 5,25 & no & yes & SYM \\
\hline & 8,11 & 139,60 & 138,87 & 136,75 & 137,89 & 136,15 & 136,15 & MTAR* & 3,48 & 3,23 & & & no & yes & SYM \\
\hline & 8,12 & 225,62 & 225,32 & 224,47 & 221,76 & 219,25 & 219,25 & MTAR* & 4,33 & 6,15 & & & no & yes & SYM \\
\hline & 8,13 & 107,53 & 102,36 & 104,33 & 105,36 & 94,37 & 94,37 & MTAR* & 11,43 & 16,14 & & & yes,asym & yes & MTAR* \\
\hline & 8,14 & 111,28 & 110,82 & 109,53 & 111,22 & 105,96 & 105,96 & MTAR* & 2,98 & 5,05 & & & no & yes & SYM \\
\hline & 8,15 & 117,65 & 117,04 & 115,80 & 118,01 & 114,01 & 114,01 & MTAR* & 2,54 & 3,92 & & & no & no & STD \\
\hline & 8,16 & 116,44 & 114,86 & 109,55 & 115,04 & 109,02 & 109,02 & MTAR* & 4,24 & 7,41 & & & no & no & STD \\
\hline & 8,17 & 117,46 & 115,98 & 113,58 & 117,66 & 113,50 & 113,50 & MTAR* & 2,69 & 3,85 & & & no & no & STD \\
\hline & 8,18 & 160,23 & 158,61 & 158,73 & 159,53 & 154,91 & 154,91 & MTAR* & 2,92 & 5,29 & & & no & no & STD \\
\hline \multirow[t]{15}{*}{ Lithuania } & 8,01 & 725,72 & 723,54 & 724,35 & 729,13 & 723,23 & 723,23 & MTAR* & 29,39 & 6,50 & & & yes,asym & yes & MTAR* \\
\hline & 8,02 & 219,68 & 215,98 & 217,07 & 216,89 & 213,82 & 213,82 & MTAR* & 5,13 & 5,54 & & & no & yes & SYM \\
\hline & 8,03 & 645,13 & 644,30 & 643,48 & 642,43 & 629,32 & 629,32 & MTAR* & 14,31 & 16,25 & & & yes,asym & yes & MTAR* \\
\hline & 8,04 & 228,57 & 224,87 & 225,38 & 223,37 & 223,25 & 223,25 & MTAR* & 4,67 & 5,02 & & & no & yes & SYM \\
\hline & 8,05 & 687,13 & 679,87 & 679,12 & 688,07 & 686,12 & 679,12 & BTAR* & 9,34 & 2,61 & 2,58 & 10,45 & no & yes & SYM \\
\hline & 8,06 & 229,05 & 224,00 & 224,43 & 223,20 & 223,18 & 223,18 & MTAR* & 4,57 & 5,64 & & & no & yes & SYM \\
\hline & 8,07 & 750,78 & 749,60 & 747,13 & 746,62 & 743,09 & 743,09 & MTAR* & 10,82 & 7,73 & & & yes,asym & yes & MTAR* \\
\hline & 8,08 & 232,37 & 227,11 & 227,17 & 230,43 & 227,81 & 227,11 & TAR* & 4,31 & 5,04 & & & no & yes & SYM \\
\hline & 8,09 & 837,53 & 837,55 & 839,55 & 837,31 & 837,55 & 837,31 & MTAR0 & 4,49 & 0,23 & & & no & yes & SYM \\
\hline & 8,10 & 221,62 & 214,47 & 210,55 & 220,01 & 211,71 & 210,55 & BTAR* & 5,13 & 12,89 & 0,07 & 12,88 & no & no & STD \\
\hline & 8,11 & 403,91 & 401,33 & 396,71 & 402,57 & 389,88 & 389,88 & MTAR* & 9,31 & 14,30 & & & no & yes & SYM \\
\hline & 8,12 & 229,09 & 223,84 & 223,77 & 226,01 & 224,66 & 223,77 & BTAR* & 3,12 & 1,84 & 1,42 & 6,83 & no & no & STD \\
\hline & 8,13 & 629,73 & 628,00 & 626,49 & 629,30 & 625,89 & 625,89 & MTAR* & 13,79 & 3,84 & & & yes,asym & yes & MTAR* \\
\hline & 8,14 & 224,66 & 220,00 & 220,86 & 219,40 & 219,40 & 219,40 & MTAR0 & 4,29 & 4,99 & & & no & yes & SYM \\
\hline & 8,15 & 430,99 & 421,11 & 417,19 & 423,99 & 409,41 & 409,41 & MTAR* & 22,25 & 30,92 & & & yes,asym & yes & MTAR* \\
\hline \multirow[t]{12}{*}{ Poland } & 8,01 & 324,35 & 323,14 & 317,96 & 323,59 & 320,88 & 317,96 & BTAR* & 5,43 & 7,20 & 0,27 & 7,62 & no & yes & SYM \\
\hline & 8,02 & 331,85 & 330,83 & 326,96 & 331,33 & 328,95 & 326,96 & BTAR* & 5,05 & 6,23 & 0,03 & 5,89 & no & yes & SYM \\
\hline & 8,03 & 336,79 & 335,41 & 332,67 & 335,72 & 332,86 & 332,67 & BTAR* & 4,70 & 5,77 & 0,46 & 5,76 & no & yes & SYM \\
\hline & 8,04 & 343,76 & 341,86 & 337,19 & 341,48 & 339,07 & 337,19 & BTAR* & 5,38 & 8,57 & 0,00 & 6,32 & no & yes & SYM \\
\hline & 8,05 & 345,71 & 343,18 & 337,57 & 344,17 & 339,46 & 337,57 & BTAR* & 6,51 & 10,93 & 0,24 & 7,25 & no & yes & SYM \\
\hline & 8,06 & 257,48 & 256,88 & 258,13 & 257,30 & 255,83 & 255,83 & MTAR* & 3,66 & 1,54 & & & no & yes & SYM \\
\hline & 8,07 & 320,09 & 319,48 & 319,90 & 318,73 & 315,90 & 315,90 & MTAR* & 7,84 & 4,00 & & & no & yes & SYM \\
\hline & 8,08 & 332,48 & 331,57 & 328,29 & 332,11 & 329,53 & 328,29 & BTAR* & 4,96 & 5,55 & 0,08 & 5,59 & no & yes & SYM \\
\hline & 8,09 & 339,54 & 338,33 & 334,95 & 339,03 & 336,22 & 334,95 & BTAR* & 5,00 & 6,36 & 0,24 & 5,89 & no & yes & SYM \\
\hline & 8,10 & 345,24 & 343,22 & 316,53 & 344,23 & 340,22 & 316,53 & BTAR* & 15,89 & 34,46 & 1,52 & 34,53 & yes,asym & yes & BTAR* \\
\hline & 8,11 & 345,14 & 343,08 & 333,75 & 342,44 & 341,15 & 333,75 & BTAR* & 7,71 & 13,06 & 0,00 & 11,16 & no & yes & SYM \\
\hline & 8,12 & 260,08 & 257,87 & 248,02 & 258,63 & 255,91 & 248,02 & BTAR* & 8,16 & 13,99 & 1,49 & 13,82 & no & yes & SYM \\
\hline \multirow[t]{7}{*}{ Slovak Republic } & 8,01 & 419,94 & 411,65 & 402,96 & 421,81 & 405,17 & 402,96 & BTAR* & 7,84 & 19,36 & 3,46 & 19,49 & no & no & STD \\
\hline & 8,02 & 437,91 & 430,75 & 432,21 & 438,84 & 423,73 & 423,73 & MTAR* & 9,34 & 16,54 & & & no & no & STD \\
\hline & 8,03 & 410,84 & 407,18 & 408,65 & 411,79 & 406,26 & 406,26 & MTAR* & 3,49 & 5,36 & & & no & no & STD \\
\hline & 8,04 & 315,34 & 313,99 & 314,70 & 315,99 & 313,45 & 313,45 & MTAR* & 2,42 & 2,58 & & & no & no & STD \\
\hline & 8,05 & 347,59 & 346,68 & 347,97 & 349,31 & 347,25 & 346,68 & TAR* & 4,16 & 3,27 & & & no & yes & SYM \\
\hline & 8,06 & 381,52 & 378,47 & 373,69 & 378,10 & 377,57 & 373,69 & BTAR* & 6,66 & 9,98 & 5,68 & 8,40 & no & no & STD \\
\hline & 8,07 & 299,61 & 294,17 & 224,74 & 304,08 & 293,24 & 224,74 & BTAR* & 43,46 & 0,10 & 122,02 & 116,75 & yes,asym & no & BTAR* $^{*}$ \\
\hline
\end{tabular}

Note: Asymmetric cointegration is established based on the critical values reported by Enders and Siklos (2000) for 50 observations and the 1\% significancelevel. Regarding the symmetric Engle-Granger cointegration decision see table B2. The STD_LL model is applied to all rates from Slovenia because these rates were found to be $\mathrm{I}(0)$. As such, no results for Slovenia are reported in this table. 
Table B4: 5-year rolling symmetric cointegration of bank rate and money market ratı

\begin{tabular}{|c|c|c|c|c|c|c|c|c|c|c|c|c|}
\hline \multirow{2}{*}{ country } & \multirow[b]{2}{*}{ rate } & \multirow{2}{*}{$\begin{array}{c}\text { obser- } \\
\text { vations }\end{array}$} & \multicolumn{2}{|c|}{ rolling sample } & \multicolumn{2}{|c|}{ intercept } & \multicolumn{2}{|c|}{ slope } & & & & cointe- \\
\hline & & & from & to & coefficient & t-statistic & coefficient & $\overline{\text { t-statistic }}$ & DW & DF & $\mathrm{ADF}$ & gration? \\
\hline Panel A: Short-te & m corp & ate loan 1 & & & & & & & & & & \\
\hline Czech Republic & 4 & 36 & Jan-99 & Dec-03 & 1,17 & 21,79 & 0,95 & 69,05 & 0,96 & $-3,24$ & & yes \\
\hline Estonia & 4 & 57 & Jan-97 & Dec-01 & 7,18 & 13,16 & 0,66 & 10,19 & 0,57 & $-3,11$ & & yes \\
\hline & 4 & 60 & Jan-98 & Dec-02 & 6,11 & 13,09 & 0,72 & 11,87 & 0,61 & $-3,31$ & & yes \\
\hline & 4 & 60 & Jan-99 & Dec-03 & 5,19 & 11,21 & 0,84 & 9,29 & 0,70 & $-3,67$ & & yes \\
\hline Hungary & 4 & 60 & Jan-95 & Dec-99 & $-4,92$ & $-6,75$ & 1,30 & 39,75 & 0,24 & $-2,46$ & & no \\
\hline & 4 & 60 & Jan-96 & Dec-00 & 0,20 & 0,55 & 1,04 & 54,72 & 0,36 & $-3,16$ & & yes \\
\hline & 4 & 60 & Jan-97 & Dec-01 & 1,82 & 9,57 & 0,93 & 79,14 & 0,84 & $-4,25$ & $-4,90$ & yes \\
\hline & 4 & 60 & Jan-98 & Dec-02 & 1,91 & 11,01 & 0,92 & 72,78 & 0,80 & $-3,90$ & $-5,08$ & yes \\
\hline & 4 & 60 & Jan-99 & Dec-03 & 1,90 & 10,84 & 0,92 & 60,40 & 1,00 & $-4,42$ & $-5,47$ & yes \\
\hline Latvia & 4 & 60 & Jan-99 & Dec-03 & 4,12 & 3,59 & 1,43 & 5,37 & 0,37 & $-2,58$ & & no \\
\hline Lithuania & 4 & 60 & Jan-99 & Dec-03 & 6,03 & 9,59 & 1,13 & 7,18 & 0,60 & $-3,46$ & & yes \\
\hline Poland & 4 & 49 & Jan-96 & Dec-00 & 5,81 & 5,33 & 0,85 & 14,90 & 1,26 & $-4,65$ & & yes \\
\hline & 4 & 60 & Jan-97 & Dec-01 & 5,27 & 5,52 & 0,86 & 16,80 & 1,18 & $-5,00$ & $-2,77$ & yes \\
\hline & 4 & 60 & Jan-98 & Dec-02 & 3,51 & 5,40 & 0,95 & 23,72 & 1,11 & $-5,09$ & $-2,39$ & yes \\
\hline & 4 & 54 & Jan-99 & Dec-03 & 3,00 & 6,48 & 0,95 & 29,01 & 1,42 & $-6,28$ & $-3,34$ & yes \\
\hline Slovak Republic & 4 & 50 & Jan-95 & Dec-99 & 12,89 & 10,94 & 0,35 & 4,77 & 0,28 & $-1,94$ & $-0,95$ & no \\
\hline & 4 & 56 & Jan-96 & Dec-00 & 11,48 & 10,79 & 0,42 & 6,19 & 0,31 & $-2,21$ & $-1,33$ & no \\
\hline & 4 & 56 & Jan-97 & Dec-01 & 9,70 & 10,04 & 0,53 & 8,30 & 0,31 & $-2,56$ & $-1,79$ & no \\
\hline & 4 & 60 & Jan-98 & Dec-02 & 6,11 & 7,56 & 0,82 & 12,84 & 0,54 & $-3,05$ & $-2,66$ & yes \\
\hline & 4 & 60 & Jan-99 & Dec-03 & 1,00 & 1,32 & 1,34 & 16,91 & 0,71 & $-3,61$ & $-1,47$ & yes \\
\hline Panel B: Long-ter & n corp & ate loan $\mathrm{r}$ & & & & & & & & & & \\
\hline Estonia & 5 & 57 & Jan-97 & Dec-01 & 7,28 & 16,84 & 0,48 & 9,31 & 0,96 & $-4,31$ & & yes \\
\hline & 5 & 60 & Jan-98 & Dec-02 & 6,61 & 19,62 & 0,54 & 12,42 & 1,25 & $-5,26$ & & yes \\
\hline & 5 & 60 & Jan-99 & Dec-03 & 5,70 & 15,65 & 0,63 & 8,76 & 1,20 & $-5,40$ & $-1,77$ & yes \\
\hline Hungary & 5 & 60 & Jan-95 & Dec-99 & $-2,82$ & $-4,43$ & 1,24 & 43,31 & 0,89 & $-4,73$ & $-2,70$ & yes \\
\hline & 5 & 60 & Jan-96 & Dec-00 & 0,49 & 1,28 & 1,07 & 53,35 & 1,17 & $-4,90$ & & yes \\
\hline & 5 & 60 & Jan-97 & Dec-01 & 1,47 & 5,56 & 1,00 & 61,18 & 1,01 & $-5,08$ & & yes \\
\hline & 5 & 60 & Jan-98 & Dec-02 & 1,71 & 7,61 & 0,98 & 59,51 & 1,04 & $-4,62$ & & yes \\
\hline & 5 & 60 & Jan-99 & Dec-03 & 2,05 & 5,19 & 0,95 & 27,47 & 0,98 & $-4,35$ & $-4,38$ & yes \\
\hline Latvia & 5 & 60 & Jan-99 & Dec-03 & 5,51 & 7,63 & 1,05 & 6,25 & 0,55 & $-3,70$ & $-2,95$ & yes \\
\hline Lithuania & 5,01 & 60 & Jan-99 & Dec-03 & 5,91 & 10,75 & 1,00 & 7,25 & 0,74 & $-4,26$ & & yes \\
\hline & 5,02 & 60 & Jan-99 & Dec-03 & 5,95 & 10,84 & 0,83 & 6,05 & 0,56 & $-3,04$ & $-2,42$ & yes \\
\hline Poland & 5,01 & 60 & Jan-97 & Dec-01 & 5,62 & 5,61 & 0,86 & 15,90 & 1,06 & $-4,66$ & $-2,66$ & yes \\
\hline & 5,01 & 60 & Jan-98 & Dec-02 & 4,50 & 6,69 & 0,91 & 21,85 & 0,96 & $-4,38$ & $-2,18$ & yes \\
\hline & 5,01 & 54 & Jan-99 & Dec-03 & 3,99 & 7,97 & 0,90 & 25,47 & 1,19 & $-6,02$ & & yes \\
\hline & 5,02 & 60 & Jan-98 & Dec-02 & 6,14 & 11,59 & 0,81 & 24,66 & 1,41 & $-5,86$ & $-3,04$ & yes \\
\hline & 5,02 & 54 & Jan-99 & Dec-03 & 4,39 & 8,22 & 0,90 & 23,69 & 1,13 & $-5,25$ & & yes \\
\hline Panel C: Consum & r loan & & & & & & & & & & & \\
\hline Czech Republic & 3 & 60 & Jan-93 & Dec-97 & 13,28 & 25,32 & $-0,01$ & $-0,37$ & 1,63 & $-7,28$ & & yes \\
\hline & 3 & 60 & Jan-94 & Dec-98 & 13,51 & 31,08 & $-0,06$ & $-1,95$ & 1,32 & $-5,50$ & $-2,69$ & yes \\
\hline & 3 & 60 & Jan-95 & Dec-99 & 10,81 & 22,83 & 0,10 & 2,83 & 0,30 & $-2,38$ & $-0,93$ & no \\
\hline & 3 & 60 & Jan-96 & Dec-00 & 9,26 & 29,49 & 0,18 & 7,11 & 0,46 & $-3,34$ & $-2,44$ & yes \\
\hline & 3 & 60 & Jan-97 & Dec-01 & 8,75 & 53,83 & 0,18 & 12,88 & 1,11 & $-5,17$ & $-4,00$ & yes \\
\hline & 3 & 60 & Jan-98 & Dec-02 & 8,26 & 92,33 & 0,22 & 19,84 & 1,48 & $-5,85$ & & yes \\
\hline & 3 & 60 & Jan-99 & Dec-03 & 8,28 & 54,71 & 0,23 & 7,46 & 0,91 & $-4,11$ & & yes \\
\hline Estonia & 3 & 57 & Jan-97 & Dec-01 & 12,09 & 22,92 & 0,14 & 2,31 & 0,79 & $-3,80$ & & yes \\
\hline & 3 & 60 & Jan-98 & Dec-02 & 11,19 & 23,82 & 0,24 & 3,92 & 0,85 & $-3,78$ & $-4,40$ & yes \\
\hline & 3 & 60 & Jan-99 & Dec-03 & 10,88 & 22,55 & 0,27 & 2,85 & 0,87 & $-4,11$ & $-5,15$ & yes \\
\hline Hungary & 3 & 60 & Jan-95 & Dec-99 & 12,96 & 30,61 & 0,67 & 35,24 & 0,62 & $-3,34$ & & yes \\
\hline & 3 & 60 & Jan-96 & Dec-00 & 14,33 & 43,97 & 0,60 & 35,56 & 0,55 & $-3,34$ & & yes \\
\hline & 3 & 60 & Jan-97 & Dec-01 & 17,27 & 46,22 & 0,43 & 18,76 & 0,49 & $-1,78$ & & yes \\
\hline & 3 & 60 & Jan-98 & Dec-02 & 19,08 & 45,51 & 0,31 & 10,27 & 0,42 & $-2,53$ & & yes \\
\hline & 3 & 60 & Jan-99 & Dec-03 & 19,32 & 39,85 & 0,27 & 6,40 & 0,40 & $-2,63$ & & yes \\
\hline Latvia & 3,01 & 60 & Jan-99 & Dec-03 & 11,25 & 15,31 & 0,82 & 4,82 & 0,71 & $-3,46$ & & yes \\
\hline & 3,02 & 60 & Jan-99 & Dec-03 & 1,93 & 0,96 & 2,76 & 5,88 & 0,41 & $-2,60$ & $-0,99$ & yes \\
\hline Lithuania & 3,01 & 60 & Jan-99 & Dec-03 & 9,26 & 15,70 & 0,82 & 5,53 & 0,92 & $-4,34$ & & yes \\
\hline & 3,02 & 60 & Jan-99 & Dec-03 & 9,59 & 22,56 & 0,77 & 7,24 & 0,56 & $-3,19$ & & yes \\
\hline Poland & 3 & 60 & Jan-97 & Dec-01 & 12,31 & 12,17 & 0,61 & 11,23 & 0,57 & $-3,11$ & $-1,62$ & yes \\
\hline & 3 & 60 & Jan-98 & Dec-02 & 12,16 & 16,55 & 0,62 & 13,65 & 0,43 & $-2,67$ & $-1,91$ & yes \\
\hline & 3 & 54 & Jan-99 & Dec-03 & 13,92 & 35,23 & 0,46 & 16,43 & 0,80 & $-5,17$ & & yes \\
\hline Slovak Republic & 3 & 50 & Jan-95 & Dec-99 & 13,84 & 15,34 & $-0,18$ & $-3,25$ & 0,50 & $-2,77$ & & yes \\
\hline & 3 & 56 & Jan-96 & Dec-00 & 9,34 & 13,66 & 0,05 & 1,17 & 0,44 & $-3,15$ & & yes \\
\hline & 3 & 56 & Jan-97 & Dec-01 & 7,83 & 19,83 & 0,12 & 4,57 & 1,03 & $-4,32$ & & yes \\
\hline & 3 & 60 & Jan-98 & Dec-02 & 8,83 & 24,93 & 0,05 & 1,65 & 0,69 & $-4,73$ & $-3,16$ & yes \\
\hline & 3 & 60 & Jan-99 & Dec-03 & 9,93 & 29,76 & $-0,08$ & $-2,18$ & 0,57 & $-3,20$ & & yes \\
\hline Panel D: Mortgag & rates & & & & & & & & & & & \\
\hline Estonia & 2 & 57 & Jan-97 & Dec-01 & 11,12 & 49,58 & 0,13 & 4,91 & 0,80 & $-3,92$ & & yes \\
\hline & 2 & 60 & Jan-98 & Dec-02 & 9,98 & 36,63 & 0,23 & 6,39 & 0,63 & $-2,59$ & $-2,60$ & yes \\
\hline & 2 & 60 & Jan-99 & Dec-03 & 6,50 & 9,35 & 0,73 & 5,33 & 0,21 & $-2,86$ & $-1,45$ & no \\
\hline Hungary & 2 & 60 & Jan-95 & Dec-99 & 14,89 & 15,58 & 0,61 & 14,21 & 0,24 & $-2,09$ & $-2,78$ & no \\
\hline
\end{tabular}


Table B4: 5-year rolling symmetric cointegration of bank rate and money market ratı

\begin{tabular}{|c|c|c|c|c|c|c|c|c|c|c|c|c|}
\hline \multirow[b]{2}{*}{ country } & \multirow[b]{2}{*}{ rate } & \multirow{2}{*}{$\begin{array}{c}\text { obser- } \\
\text { vations }\end{array}$} & \multicolumn{2}{|c|}{ rolling sample } & \multicolumn{2}{|c|}{ intercept } & \multicolumn{2}{|c|}{ slope } & & & & cointe- \\
\hline & & & from & to & coefficient & $\overline{\text { t-statistic }}$ & coefficient & $\overline{\text { t-statistic }}$ & DW & DF & $\mathrm{ADF}$ & gration? \\
\hline & 2 & 60 & Jan-96 & Dec-00 & 8,72 & 12,98 & 0,92 & 26,25 & 0,32 & $-2,59$ & & no \\
\hline & 2 & 60 & Jan-97 & Dec-01 & 4,54 & 7,27 & 1,16 & 30,15 & 0,39 & $-2,57$ & & yes \\
\hline & 2 & 60 & Jan-98 & Dec-02 & 2,81 & 5,06 & 1,29 & 31,79 & 0,44 & $-3,02$ & & yes \\
\hline & 2 & 60 & Jan-99 & Dec-03 & 2,42 & 2,55 & 1,30 & 15,75 & 0,31 & $-0,63$ & $-1,38$ & no \\
\hline Latvia & 2 & 60 & Jan-99 & Dec-03 & 7,55 & 8,43 & 1,01 & 4,88 & 0,34 & $-2,31$ & $-1,91$ & no \\
\hline Lithuania & 2,01 & 60 & Jan-99 & Dec-03 & 5,73 & 7,84 & 1,24 & 6,77 & 0,58 & $-3,47$ & & yes \\
\hline & 2,02 & 60 & Jan-99 & Dec-03 & 6,51 & 14,01 & 0,06 & 0,55 & 0,34 & $-2,27$ & $-1,27$ & no \\
\hline Panel E: Current & ccount & eposit rat & & & & & & & & & & \\
\hline Hungary & 7 & 60 & Jan-95 & Dec-99 & 3,57 & 8,93 & 0,19 & 10,71 & 0,18 & $-1,54$ & $-1,96$ & no \\
\hline & 7 & 60 & Jan-96 & Dec-00 & 0,21 & 0,64 & 0,36 & 20,60 & 0,19 & $-2,36$ & $-2,03$ & no \\
\hline & 7 & 60 & Jan-97 & Dec-01 & $-1,67$ & $-6,55$ & 0,47 & 29,91 & 0,33 & $-2,28$ & $-2,01$ & no \\
\hline & 7 & 60 & Jan-98 & Dec-02 & $-1,91$ & $-8,52$ & 0,49 & 29,73 & 0,23 & $-2,42$ & & no \\
\hline & 7 & 60 & Jan-99 & Dec-03 & $-2,29$ & $-7,21$ & 0,52 & 18,97 & 0,38 & $-1,16$ & $-2,35$ & no \\
\hline Poland & 7,01 & 60 & Jan-97 & Dec-01 & $-2,50$ & $-2,06$ & 0,63 & 9,68 & 0,35 & $-2,48$ & $-1,42$ & no \\
\hline & 7,01 & 60 & Jan-98 & Dec-02 & $-3,63$ & $-4,18$ & 0,67 & 12,49 & 0,29 & $-2,19$ & $-1,23$ & no \\
\hline & 7,01 & 54 & Jan-99 & Dec-03 & $-2,40$ & $-3,85$ & 0,55 & 12,39 & 0,25 & $-2,43$ & $-1,87$ & no \\
\hline & 7,02 & 60 & Jan-97 & Dec-01 & 0,22 & 0,29 & 0,32 & 7,67 & 0,25 & $-1,64$ & $-1,12$ & no \\
\hline & 7,02 & 60 & Jan-98 & Dec-02 & $-2,50$ & $-3,93$ & 0,46 & 11,65 & 0,24 & $-2,11$ & $-1,31$ & no \\
\hline & 7,02 & 54 & Jan-99 & Dec-03 & $-2,35$ & $-4,37$ & 0,43 & 11,34 & 0,20 & $-2,38$ & $-1,84$ & no \\
\hline Panel F: Savings & ccoun & & & & & & & & & & & \\
\hline Czech Republic & 9 & 60 & Jan-93 & Dec-97 & 2,69 & 44,17 & $-0,02$ & $-4,37$ & 0,69 & $-4,42$ & $-3,32$ & yes \\
\hline & 9 & 60 & Jan-94 & Dec-98 & 2,72 & 30,63 & $-0,03$ & $-3,99$ & 0,40 & $-1,88$ & $-0,47$ & yes \\
\hline & 9 & 60 & Jan-95 & Dec-99 & 2,08 & 19,02 & 0,01 & 1,50 & 0,10 & $-0,89$ & $-0,54$ & no \\
\hline & 9 & 60 & Jan-96 & Dec-00 & 1,67 & 19,99 & 0,03 & 5,04 & 0,21 & $-2,01$ & $-1,78$ & no \\
\hline & 9 & 60 & Jan-97 & Dec-01 & 1,49 & 35,40 & 0,04 & 9,82 & 0,68 & $-4,23$ & $-1,97$ & yes \\
\hline & 9 & 60 & Jan-98 & Dec-02 & 1,27 & 34,11 & 0,06 & 12,12 & 0,33 & $-1,68$ & $-0,71$ & no \\
\hline & 9 & 60 & Jan-99 & Dec-03 & 0,51 & 9,47 & 0,20 & 18,09 & 0,32 & $-3,15$ & & no \\
\hline Lithuania & 9 & 60 & Jan-99 & Dec-03 & 3,00 & 6,88 & 0,56 & 5,14 & 0,71 & $-3,47$ & $-1,97$ & yes \\
\hline Slovak Republic & 9 & 50 & Jan-95 & Dec-99 & 3,19 & 21,66 & 0,02 & 1,84 & 0,18 & $-0,96$ & 0,05 & no \\
\hline & 9 & 56 & Jan-96 & Dec-00 & 3,33 & 24,30 & 0,01 & 1,12 & 0,15 & $-1,06$ & $-0,55$ & no \\
\hline & 9 & 56 & Jan-97 & Dec-01 & 2,87 & 18,52 & 0,04 & 3,54 & 0,13 & $-1,03$ & & no \\
\hline & 9 & 60 & Jan-98 & Dec-02 & 2,13 & 12,29 & 0,09 & 6,45 & 0,22 & $-1,41$ & $-0,15$ & no \\
\hline & 9 & 60 & Jan-99 & Dec-03 & 0,83 & 3,62 & 0,21 & 8,70 & 0,26 & $-2,07$ & $-0,93$ & no \\
\hline Panel G: Time de & osit ra & & & & & & & & & & & \\
\hline Czech Republic & 8,01 & 60 & Jan-93 & Dec-97 & 7,42 & 33,29 & 0,20 & 11,50 & 1,11 & $-4,76$ & $-2,85$ & yes \\
\hline & 8,01 & 60 & Jan-94 & Dec-98 & 7,07 & 25,50 & 0,25 & 11,85 & 0,77 & $-3,77$ & $-2,08$ & yes \\
\hline & 8,01 & 60 & Jan-95 & Dec-99 & 4,32 & 10,38 & 0,42 & 13,18 & 0,50 & $-2,67$ & & yes \\
\hline & 8,01 & 60 & Jan-96 & Dec-00 & 2,73 & 7,36 & 0,51 & 17,02 & 0,64 & $-3,34$ & $-2,64$ & yes \\
\hline & 8,01 & 60 & Jan-97 & Dec-01 & 1,95 & 5,91 & 0,55 & 18,82 & 0,68 & $-3,40$ & $-2,50$ & yes \\
\hline & 8,01 & 60 & Jan-98 & Dec-02 & $-0,08$ & $-0,72$ & 0,80 & 59,23 & 0,27 & $-3,07$ & & no \\
\hline & 8,01 & 60 & Jan-99 & Dec-03 & $-0,75$ & $-7,92$ & 0,94 & 48,90 & 0,33 & $-3,78$ & $-2,03$ & yes \\
\hline & 8,02 & 60 & Jan-93 & Dec-97 & 11,10 & 39,40 & 0,00 & $-0,17$ & 0,20 & $-1,81$ & & no \\
\hline & 8,02 & 60 & Jan-94 & Dec-98 & 10,90 & 36,73 & 0,01 & 0,52 & 0,16 & $-1,72$ & & no \\
\hline & 8,02 & 60 & Jan-95 & Dec-99 & 6,38 & 10,51 & 0,29 & 6,31 & 0,27 & $-1,90$ & $-1,49$ & no \\
\hline & 8,02 & 60 & Jan-96 & Dec-00 & 3,83 & 7,21 & 0,43 & 10,12 & 0,47 & $-2,82$ & $-2,16$ & yes \\
\hline & 8,02 & 60 & Jan-97 & Dec-01 & 2,55 & 5,39 & 0,49 & 11,82 & 0,55 & $-2,99$ & $-1,69$ & yes \\
\hline & 8,02 & 60 & Jan-98 & Dec-02 & $-0,13$ & $-0,64$ & 0,83 & 32,22 & 0,39 & $-2,79$ & & yes \\
\hline & 8,02 & 60 & Jan-99 & Dec-03 & $-0,90$ & $-4,01$ & 0,98 & 21,52 & 0,49 & $-5,45$ & $-1,64$ & yes \\
\hline & 8,03 & 60 & Jan-93 & Dec-97 & 14,24 & 12,91 & $-0,36$ & $-4,24$ & 0,18 & $-1,90$ & $-1,51$ & no \\
\hline & 8,03 & 60 & Jan-94 & Dec-98 & 13,74 & 13,80 & $-0,45$ & $-6,07$ & 0,35 & $-2,08$ & $-0,56$ & no \\
\hline & 8,03 & 60 & Jan-95 & Dec-99 & 6,26 & 7,10 & 0,00 & $-0,01$ & 0,01 & $-2,09$ & $-1,90$ & no \\
\hline & 8,03 & 60 & Jan-96 & Dec-00 & 3,62 & 10,05 & 0,12 & 3,97 & 0,11 & $-3,39$ & $-3,43$ & yes \\
\hline & 8,03 & 60 & Jan-97 & Dec-01 & 3,03 & 27,30 & 0,12 & 11,81 & 0,68 & $-4,28$ & $-1,67$ & yes \\
\hline & 8,03 & 60 & Jan-98 & Dec-02 & 2,79 & 90,13 & 0,13 & 34,19 & 0,26 & $-2,27$ & $-1,72$ & no \\
\hline & 8,03 & 60 & Jan-99 & Dec-03 & 2,66 & 71,01 & 0,16 & 20,87 & 0,16 & $-1,57$ & $-2,11$ & no \\
\hline & 8,04 & 60 & Jan-93 & Dec-97 & 9,44 & 32,52 & 0,08 & 3,35 & 0,47 & $-2,92$ & $-1,96$ & yes \\
\hline & 8,04 & 60 & Jan-94 & Dec-98 & 9,08 & 31,32 & 0,09 & 4,28 & 0,49 & $-2,79$ & $-1,85$ & yes \\
\hline & 8,04 & 60 & Jan-95 & Dec-99 & 5,01 & 10,47 & 0,34 & 9,42 & 0,27 & $-1,86$ & & no \\
\hline & 8,04 & 60 & Jan-96 & Dec-00 & 3,01 & 7,95 & 0,45 & 14,80 & 0,54 & $-3,17$ & $-2,20$ & yes \\
\hline & 8,04 & 60 & Jan-97 & Dec-01 & 2,15 & 6,82 & 0,49 & 17,49 & 0,67 & $-3,38$ & $-1,60$ & yes \\
\hline & 8,04 & 60 & Jan-98 & Dec-02 & 0,30 & 2,81 & 0,72 & 54,83 & 0,30 & $-2,84$ & & no \\
\hline & 8,04 & 60 & Jan-99 & Dec-03 & $-0,11$ & $-1,06$ & 0,81 & 36,87 & 0,30 & $-4,36$ & $-1,43$ & yes \\
\hline Estonia & 8,01 & 60 & Jan-96 & Dec-00 & 2,03 & 5,02 & 0,42 & 8,72 & 0,63 & $-3,41$ & & yes \\
\hline & 8,01 & 60 & Jan-97 & Dec-01 & 1,56 & 4,49 & 0,45 & 10,74 & 0,67 & $-3,69$ & & yes \\
\hline & 8,01 & 60 & Jan-98 & Dec-02 & 1,50 & 5,98 & 0,42 & 13,01 & 0,48 & $-3,46$ & & yes \\
\hline & 8,01 & 60 & Jan-99 & Dec-03 & 0,92 & 7,30 & 0,52 & 21,06 & 1,32 & $-5,61$ & $-3,06$ & yes \\
\hline & 8,02 & 60 & Jan-96 & Dec-00 & 4,70 & 10,21 & 0,38 & 6,97 & 0,12 & $-1,29$ & & no \\
\hline & 8,02 & 60 & Jan-97 & Dec-01 & 2,99 & 7,41 & 0,50 & 10,30 & 0,19 & $-1,97$ & & no \\
\hline & 8,02 & 60 & Jan-98 & Dec-02 & 1,80 & 5,96 & 0,57 & 14,64 & 0,28 & $-2,12$ & $-4,06$ & yes \\
\hline & 8,02 & 60 & Jan-99 & Dec-03 & 0,81 & 4,01 & 0,71 & 17,74 & 0,34 & $-3,55$ & $-2,61$ & yes \\
\hline & 8,03 & 60 & Jan-96 & Dec-00 & 1,03 & 2,38 & 0,55 & 10,67 & 0,44 & $-2,69$ & & yes \\
\hline
\end{tabular}


Table B4: 5-year rolling symmetric cointegration of bank rate and money market ratı

\begin{tabular}{|c|c|c|c|c|c|c|c|c|c|c|c|c|}
\hline \multirow[b]{2}{*}{ country } & \multirow[b]{2}{*}{ rate } & \multirow{2}{*}{$\begin{array}{c}\text { obser- } \\
\text { vations }\end{array}$} & \multicolumn{2}{|c|}{ rolling sample } & \multicolumn{2}{|c|}{ intercept } & \multicolumn{2}{|c|}{ slope } & & & & cointe- \\
\hline & & & from & to & coefficient & $\overline{\text { t-statistic }}$ & coefficient & $\overline{\text { t-statistic }}$ & DW & DF & $\mathrm{ADF}$ & gration? \\
\hline & 8,03 & 60 & Jan-97 & Dec-01 & 0,91 & 2,25 & 0,56 & 11,46 & 0,37 & $-2,64$ & $-2,67$ & no \\
\hline & 8,03 & 60 & Jan-98 & Dec-02 & 1,05 & 4,08 & 0,50 & 15,07 & 0,27 & $-3,33$ & $-3,76$ & yes \\
\hline & 8,03 & 60 & Jan-99 & Dec-03 & 1,10 & 9,31 & 0,46 & 20,10 & 0,66 & $-3,50$ & $-2,13$ & yes \\
\hline & 8,04 & 57 & Jan-97 & Dec-01 & 2,24 & 6,39 & 0,59 & 14,21 & 0,29 & $-2,19$ & $-3,48$ & yes \\
\hline & 8,04 & 60 & Jan-98 & Dec-02 & 1,57 & 5,55 & 0,63 & 17,24 & 0,34 & $-2,33$ & $-6,03$ & yes \\
\hline & 8,04 & 60 & Jan-99 & Dec-03 & 0,63 & 4,44 & 0,77 & 27,79 & 0,86 & $-5,55$ & & yes \\
\hline & 8,05 & 60 & Jan-96 & Dec-00 & 4,89 & 12,06 & 0,50 & 10,41 & 0,29 & $-1,98$ & & no \\
\hline & 8,05 & 60 & Jan-97 & Dec-01 & 3,44 & 8,18 & 0,60 & 11,96 & 0,27 & $-1,98$ & & no \\
\hline & 8,05 & 60 & Jan-98 & Dec-02 & 2,17 & 6,24 & 0,70 & 15,47 & 0,33 & $-2,25$ & $-3,78$ & yes \\
\hline & 8,05 & 60 & Jan-99 & Dec-03 & 0,89 & 3,08 & 0,88 & 15,70 & 0,41 & $-3,67$ & & yes \\
\hline & 8,06 & 60 & Jan-96 & Dec-00 & 7,64 & 16,09 & 0,18 & 3,18 & 1,41 & $-5,76$ & $-5,78$ & yes \\
\hline & 8,06 & 60 & Jan-97 & Dec-01 & 5,87 & 14,89 & 0,30 & 6,43 & 0,56 & $-2,90$ & & yes \\
\hline & 8,06 & 60 & Jan-98 & Dec-02 & 4,39 & 10,91 & 0,42 & 7,96 & 0,34 & $-2,15$ & & no \\
\hline & 8,06 & 60 & Jan-99 & Dec-03 & 1,85 & 4,11 & 0,83 & 9,44 & 0,29 & $-2,92$ & $-2,29$ & no \\
\hline Hungary & 8,01 & 60 & Jan-95 & Dec-99 & $-0,33$ & $-0,84$ & 0,82 & 46,13 & 0,73 & $-3,59$ & $-4,35$ & yes \\
\hline & 8,01 & 60 & Jan-96 & Dec-00 & $-0,92$ & $-3,99$ & 0,85 & 71,06 & 0,67 & $-3,74$ & $-4,39$ & yes \\
\hline & 8,01 & 60 & Jan-97 & Dec-01 & $-0,77$ & $-4,10$ & 0,84 & 72,87 & 0,64 & $-3,33$ & $-3,66$ & yes \\
\hline & 8,01 & 60 & Jan-98 & Dec-02 & $-0,77$ & $-4,08$ & 0,84 & 61,20 & 0,55 & $-3,13$ & $-3,67$ & yes \\
\hline & 8,01 & 60 & Jan-99 & Dec-03 & $-0,03$ & $-0,13$ & 0,78 & 37,47 & 0,72 & $-3,88$ & $-4,93$ & yes \\
\hline & 8,02 & 60 & Jan-95 & Dec-99 & $-2,97$ & $-6,85$ & 0,98 & 50,07 & 1,00 & $-4,35$ & & yes \\
\hline & 8,02 & 60 & Jan-96 & Dec-00 & $-1,34$ & $-3,54$ & 0,90 & 45,48 & 0,80 & $-4,15$ & & yes \\
\hline & 8,02 & 60 & Jan-97 & Dec-01 & $-0,86$ & $-2,88$ & 0,87 & 47,19 & 0,71 & $-3,76$ & $-4,31$ & yes \\
\hline & 8,02 & 60 & Jan-98 & Dec-02 & 0,14 & 0,60 & 0,79 & 46,45 & 0,83 & $-3,88$ & $-4,49$ & yes \\
\hline & 8,02 & 60 & Jan-99 & Dec-03 & 0,28 & 0,70 & 0,76 & 21,69 & 0,41 & $-2,05$ & & yes \\
\hline Latvia & 8,01 & 60 & Jan-99 & Dec-03 & 0,45 & 2,83 & 0,77 & 21,04 & 1,69 & $-6,56$ & $-6,25$ & yes \\
\hline & 8,02 & 60 & Jan-99 & Dec-03 & 2,06 & 9,01 & 0,56 & 10,56 & 0,75 & $-3,61$ & $-1,07$ & yes \\
\hline & 8,03 & 60 & Jan-99 & Dec-03 & 2,89 & 8,37 & 0,54 & 6,68 & 0,73 & $-3,53$ & $-0,61$ & yes \\
\hline & 8,04 & 60 & Jan-99 & Dec-03 & 4,32 & 12,11 & 0,38 & 4,56 & 0,44 & $-2,53$ & $-1,69$ & yes \\
\hline & 8,05 & 60 & Jan-99 & Dec-03 & 1,39 & 6,38 & 0,68 & 13,46 & 0,85 & $-3,97$ & $-2,15$ & yes \\
\hline & 8,06 & 60 & Jan-99 & Dec-03 & 5,01 & 12,15 & 0,30 & 3,17 & 0,47 & $-2,63$ & $-1,77$ & yes \\
\hline & 8,07 & 60 & Jan-99 & Dec-03 & 0,42 & 2,65 & 0,78 & 20,95 & 1,67 & $-6,50$ & & yes \\
\hline & 8,08 & 60 & Jan-99 & Dec-03 & 1,73 & 7,15 & 0,64 & 11,28 & 0,88 & $-3,99$ & $-1,24$ & yes \\
\hline & 8,09 & 60 & Jan-99 & Dec-03 & 2,46 & 6,71 & 0,64 & 7,54 & 1,00 & $-4,43$ & $-0,74$ & yes \\
\hline & 8,10 & 60 & Jan-99 & Dec-03 & 4,08 & 8,22 & 0,34 & 2,97 & 1,09 & $-4,62$ & $-3,21$ & yes \\
\hline & 8,11 & 60 & Jan-99 & Dec-03 & 1,01 & 4,94 & 0,73 & 15,37 & 1,09 & $-4,66$ & & yes \\
\hline & 8,12 & 60 & Jan-99 & Dec-03 & 5,52 & 10,24 & 0,07 & 0,59 & 0,85 & $-3,96$ & $-2,23$ & yes \\
\hline & 8,13 & 60 & Jan-99 & Dec-03 & 1,68 & 7,73 & 0,47 & 9,32 & 0,59 & $-3,10$ & $-3,50$ & yes \\
\hline & 8,14 & 60 & Jan-99 & Dec-03 & 3,13 & 13,58 & 0,32 & 5,90 & 0,48 & $-2,70$ & $-1,90$ & yes \\
\hline & 8,15 & 60 & Jan-99 & Dec-03 & 3,44 & 11,28 & 0,40 & 5,59 & 0,33 & $-2,09$ & $-0,83$ & no \\
\hline & 8,16 & 60 & Jan-99 & Dec-03 & 4,55 & 13,70 & 0,35 & 4,55 & 0,25 & $-1,73$ & $-0,73$ & no \\
\hline & 8,17 & 60 & Jan-99 & Dec-03 & 3,66 & 11,58 & 0,38 & 5,21 & 0,27 & $-1,89$ & $-0,84$ & no \\
\hline & 8,18 & 60 & Jan-99 & Dec-03 & 4,61 & 11,03 & 0,46 & 4,71 & 0,34 & $-2,13$ & $-1,42$ & no \\
\hline Lithuania & 8,01 & 60 & Jan-94 & Dec-98 & $-1,40$ & $-1,78$ & 0,81 & 37,86 & 1,00 & $-4,53$ & $-5,41$ & yes \\
\hline & 8,01 & 60 & Jan-95 & Dec-99 & 0,35 & 0,76 & 0,68 & 24,51 & 2,05 & $-8,48$ & & yes \\
\hline & 8,01 & 60 & Jan-96 & Dec-00 & 0,43 & 1,24 & 0,66 & 21,50 & 1,84 & $-7,12$ & & yes \\
\hline & 8,01 & 60 & Jan-97 & Dec-01 & 0,88 & 3,00 & 0,53 & 11,72 & 1,24 & $-5,09$ & $-3,43$ & yes \\
\hline & 8,01 & 60 & Jan-98 & Dec-02 & 0,78 & 3,01 & 0,47 & 8,71 & 1,11 & $-4,92$ & & yes \\
\hline & 8,01 & 60 & Jan-99 & Dec-03 & 0,68 & 3,95 & 0,41 & 9,52 & 1,41 & $-6,06$ & & yes \\
\hline & 8,02 & 60 & Jan-99 & Dec-03 & 1,56 & 4,04 & 0,70 & 7,19 & 0,42 & $-2,72$ & & yes \\
\hline & 8,03 & 60 & Jan-94 & Dec-98 & 2,20 & 3,86 & 0,64 & 41,29 & 1,00 & $-5,21$ & & yes \\
\hline & 8,03 & 60 & Jan-95 & Dec-99 & 3,59 & 8,43 & 0,55 & 21,17 & 1,17 & $-4,88$ & & yes \\
\hline & 8,03 & 60 & Jan-96 & Dec-00 & 4,74 & 21,32 & 0,41 & 20,62 & 0,98 & $-4,33$ & & yes \\
\hline & 8,03 & 60 & Jan-97 & Dec-01 & 4,77 & 16,40 & 0,33 & 7,28 & 0,57 & $-2,96$ & & yes \\
\hline & 8,03 & 60 & Jan-98 & Dec-02 & 3,22 & 7,47 & 0,54 & 6,03 & 0,42 & $-2,20$ & & yes \\
\hline & 8,03 & 60 & Jan-99 & Dec-03 & 2,14 & 5,13 & 0,74 & 7,05 & 0,47 & $-3,01$ & & yes \\
\hline & 8,04 & 60 & Jan-99 & Dec-03 & 2,13 & 5,08 & 0,72 & 6,88 & 0,45 & $-2,81$ & & yes \\
\hline & 8,05 & 60 & Jan-94 & Dec-98 & 2,20 & 2,75 & 0,69 & 31,96 & 1,60 & $-6,22$ & $-6,21$ & yes \\
\hline & 8,05 & 60 & Jan-95 & Dec-99 & 3,58 & 7,71 & 0,59 & 21,08 & 1,55 & $-6,04$ & & yes \\
\hline & 8,05 & 60 & Jan-96 & Dec-00 & 4,84 & 14,21 & 0,47 & 15,43 & 1,10 & $-4,71$ & & yes \\
\hline & 8,05 & 60 & Jan-97 & Dec-01 & 5,35 & 17,98 & 0,32 & 6,89 & 0,45 & $-2,42$ & & yes \\
\hline & 8,05 & 60 & Jan-98 & Dec-02 & 3,97 & 8,95 & 0,49 & 5,24 & 0,35 & $-1,88$ & & no \\
\hline & 8,05 & 60 & Jan-99 & Dec-03 & 2,64 & 6,11 & 0,75 & 6,90 & 0,44 & $-2,77$ & & yes \\
\hline & 8,06 & 60 & Jan-99 & Dec-03 & 2,63 & 6,14 & 0,74 & 6,87 & 0,43 & $-2,71$ & & yes \\
\hline & 8,07 & 60 & Jan-94 & Dec-98 & 2,31 & 2,70 & 0,69 & 29,88 & 1,51 & $-5,97$ & $-4,80$ & yes \\
\hline & 8,07 & 60 & Jan-95 & Dec-99 & 5,12 & 8,74 & 0,48 & 13,47 & 1,45 & $-5,87$ & $-5,71$ & yes \\
\hline & 8,07 & 60 & Jan-96 & Dec-00 & 6,09 & 13,54 & 0,37 & 9,24 & 1,38 & $-8,96$ & $-6,18$ & yes \\
\hline & 8,07 & 60 & Jan-97 & Dec-01 & 5,92 & 19,77 & 0,33 & 7,18 & 0,46 & $-2,41$ & & yes \\
\hline & 8,07 & 60 & Jan-98 & Dec-02 & 4,53 & 10,27 & 0,50 & 5,41 & 0,31 & $-1,76$ & & no \\
\hline & 8,07 & 60 & Jan-99 & Dec-03 & 3,23 & 7,37 & 0,75 & 6,81 & 0,44 & $-2,73$ & & yes \\
\hline & 8,08 & 60 & Jan-99 & Dec-03 & 3,17 & 7,27 & 0,75 & 6,88 & 0,43 & $-2,71$ & & yes \\
\hline & 8,09 & 60 & Jan-94 & Dec-98 & 3,99 & 3,54 & 0,52 & 16,96 & 1,43 & $-6,48$ & $-5,83$ & yes \\
\hline
\end{tabular}


Table B4: 5-year rolling symmetric cointegration of bank rate and money market ratı

\begin{tabular}{|c|c|c|c|c|c|c|c|c|c|c|c|c|}
\hline \multirow{2}{*}{ country } & \multirow[b]{2}{*}{ rate } & \multirow{2}{*}{$\begin{array}{c}\text { obser- } \\
\text { vations }\end{array}$} & \multicolumn{2}{|c|}{ rolling sample } & \multicolumn{2}{|c|}{ intercept } & \multicolumn{2}{|c|}{ slope } & & & & cointe- \\
\hline & & & from & to & coefficient & t-statistic & coefficient & $\overline{\text { t-statistic }}$ & DW & DF & $\mathrm{ADF}$ & gration? \\
\hline & 8,09 & 60 & Jan-95 & Dec-99 & 4,90 & 4,95 & 0,46 & 7,62 & 1,45 & $-5,94$ & $-3,76$ & yes \\
\hline & 8,09 & 60 & Jan-96 & Dec-00 & 7,70 & 21,47 & 0,14 & 4,51 & 0,85 & $-4,35$ & $-4,83$ & yes \\
\hline & 8,09 & 60 & Jan-97 & Dec-01 & 6,88 & 20,99 & 0,23 & 4,54 & 0,36 & $-2,12$ & & no \\
\hline & 8,09 & 60 & Jan-98 & Dec-02 & 5,52 & 12,01 & 0,39 & 4,09 & 0,23 & $-1,38$ & & no \\
\hline & 8,09 & 60 & Jan-99 & Dec-03 & 4,10 & 8,95 & 0,67 & 5,83 & 0,32 & $-2,16$ & & no \\
\hline & 8,10 & 60 & Jan-99 & Dec-03 & 4,04 & 8,88 & 0,69 & 6,03 & 0,32 & $-2,15$ & & no \\
\hline & 8,11 & 60 & Jan-97 & Dec-01 & 6,72 & 15,52 & 0,17 & 2,62 & 1,15 & $-5,49$ & & yes \\
\hline & 8,11 & 60 & Jan-98 & Dec-02 & 5,07 & 9,85 & 0,38 & 3,53 & 0,89 & $-3,96$ & & yes \\
\hline & 8,11 & 60 & Jan-99 & Dec-03 & 3,78 & 7,46 & 0,60 & 4,72 & 0,81 & $-3,87$ & & yes \\
\hline & 8,12 & 60 & Jan-99 & Dec-03 & 3,75 & 8,06 & 0,69 & 5,88 & 0,35 & $-2,34$ & & no \\
\hline & 8,13 & 60 & Jan-94 & Dec-98 & 1,62 & 2,93 & 0,67 & 44,41 & 1,11 & $-5,19$ & & yes \\
\hline & 8,13 & 60 & Jan-95 & Dec-99 & 2,02 & 4,96 & 0,62 & 25,08 & 1,27 & $-5,24$ & & yes \\
\hline & 8,13 & 60 & Jan-96 & Dec-00 & 2,34 & 9,09 & 0,54 & 23,82 & 1,07 & $-4,60$ & & yes \\
\hline & 8,13 & 60 & Jan-97 & Dec-01 & 2,05 & 6,92 & 0,53 & 11,65 & 1,07 & $-4,64$ & & yes \\
\hline & 8,13 & 60 & Jan-98 & Dec-02 & 1,44 & 4,54 & 0,57 & 8,56 & 0,81 & $-3,95$ & & yes \\
\hline & 8,13 & 60 & Jan-99 & Dec-03 & 1,07 & 4,38 & 0,55 & 8,94 & 0,81 & $-4,25$ & & yes \\
\hline & 8,14 & 60 & Jan-99 & Dec-03 & 2,57 & 6,16 & 0,72 & 6,86 & 0,41 & $-2,64$ & & yes \\
\hline & 8,15 & 60 & Jan-94 & Dec-98 & 1,58 & 6,14 & 0,18 & 25,36 & 1,17 & $-4,93$ & $-2,82$ & yes \\
\hline & 8,15 & 60 & Jan-95 & Dec-99 & 0,63 & 3,30 & 0,23 & 19,91 & 0,84 & $-3,96$ & $-5,10$ & yes \\
\hline & 8,15 & 60 & Jan-96 & Dec-00 & 0,76 & 5,37 & 0,21 & 16,35 & 0,69 & $-3,44$ & $-4,09$ & yes \\
\hline & 8,15 & 60 & Jan-97 & Dec-01 & 1,23 & 12,23 & 0,10 & 6,19 & 0,61 & $-3,03$ & & yes \\
\hline & 8,15 & 60 & Jan-98 & Dec-02 & 0,67 & 4,79 & 0,17 & 5,92 & 0,59 & $-2,84$ & & yes \\
\hline & 8,15 & 60 & Jan-99 & Dec-03 & 0,37 & 2,90 & 0,20 & 6,48 & 0,47 & $-3,66$ & & yes \\
\hline Poland & 8,01 & 60 & Jan-97 & Dec-01 & 0,64 & 0,86 & 0,72 & 17,91 & 1,23 & $-5,08$ & $-2,60$ & yes \\
\hline & 8,01 & 60 & Jan-98 & Dec-02 & $-1,61$ & $-3,03$ & 0,84 & 25,43 & 1,10 & $-5,12$ & $-2,68$ & yes \\
\hline & 8,01 & 54 & Jan-99 & Dec-03 & $-1,39$ & $-3,68$ & 0,80 & 30,12 & 1,42 & $-6,43$ & $-3,48$ & yes \\
\hline & 8,02 & 60 & Jan-97 & Dec-01 & 0,11 & 0,14 & 0,75 & 17,13 & 1,17 & $-4,91$ & $-2,53$ & yes \\
\hline & 8,02 & 60 & Jan-98 & Dec-02 & $-2,06$ & $-3,52$ & 0,87 & 23,94 & 1,10 & $-5,15$ & $-2,73$ & yes \\
\hline & 8,02 & 54 & Jan-99 & Dec-03 & $-1,76$ & $-4,49$ & 0,83 & 29,92 & 1,45 & $-6,11$ & $-2,16$ & yes \\
\hline & 8,03 & 60 & Jan-97 & Dec-01 & $-0,07$ & $-0,08$ & 0,79 & 16,47 & 1,08 & $-4,74$ & $-2,56$ & yes \\
\hline & 8,03 & 60 & Jan-98 & Dec-02 & $-1,87$ & $-3,03$ & 0,88 & 22,96 & 0,98 & $-4,76$ & $-2,50$ & yes \\
\hline & 8,03 & 54 & Jan-99 & Dec-03 & $-1,49$ & $-3,72$ & 0,83 & 29,13 & 1,31 & $-5,65$ & $-3,05$ & yes \\
\hline & 8,04 & 60 & Jan-97 & Dec-01 & $-0,42$ & $-0,43$ & 0,84 & 15,89 & 0,99 & $-4,50$ & $-2,54$ & yes \\
\hline & 8,04 & 60 & Jan-98 & Dec-02 & $-1,74$ & $-2,59$ & 0,90 & 21,79 & 0,87 & $-4,32$ & $-2,31$ & yes \\
\hline & 8,04 & 54 & Jan-99 & Dec-03 & $-0,98$ & $-2,34$ & 0,82 & 27,80 & 1,18 & $-5,25$ & $-2,99$ & yes \\
\hline & 8,05 & 60 & Jan-97 & Dec-01 & 0,42 & 0,42 & 0,83 & 15,43 & 1,01 & $-4,65$ & $-2,63$ & yes \\
\hline & 8,05 & 60 & Jan-98 & Dec-02 & $-1,03$ & $-1,54$ & 0,89 & 21,49 & 0,96 & $-4,61$ & $-2,44$ & yes \\
\hline & 8,05 & 54 & Jan-99 & Dec-03 & $-0,55$ & $-1,26$ & 0,83 & 26,88 & 1,34 & $-6,36$ & $-3,50$ & yes \\
\hline & 8,06 & 60 & Jan-97 & Dec-01 & 0,56 & 0,65 & 0,82 & 17,87 & 1,23 & $-5,19$ & $-2,80$ & yes \\
\hline & 8,06 & 50 & Jan-98 & Dec-02 & 0,04 & 0,04 & 0,84 & 15,82 & 1,10 & $-4,52$ & $-2,17$ & yes \\
\hline & 8,06 & 38 & Jan-99 & Dec-03 & 1,90 & 2,55 & 0,70 & 14,94 & 1,88 & $-6,22$ & & yes \\
\hline & 8,07 & 60 & Jan-97 & Dec-01 & 0,34 & 0,48 & 0,74 & 19,30 & 1,32 & $-5,46$ & & yes \\
\hline & 8,07 & 60 & Jan-98 & Dec-02 & $-1,25$ & $-2,65$ & 0,82 & 28,29 & 1,22 & $-5,46$ & $-3,01$ & yes \\
\hline & 8,07 & 54 & Jan-99 & Dec-03 & $-1,10$ & $-3,43$ & 0,79 & 35,10 & 1,84 & $-7,96$ & & yes \\
\hline & 8,08 & 60 & Jan-97 & Dec-01 & 0,56 & 0,71 & 0,76 & 17,93 & 1,23 & $-5,14$ & $-2,85$ & yes \\
\hline & 8,08 & 60 & Jan-98 & Dec-02 & $-1,49$ & $-2,75$ & 0,86 & 25,86 & 1,14 & $-5,15$ & $-2,71$ & yes \\
\hline & 8,08 & 54 & Jan-99 & Dec-03 & $-1,25$ & $-3,36$ & 0,83 & 31,39 & 1,53 & $-6,71$ & $-3,73$ & yes \\
\hline & 8,09 & 60 & Jan-97 & Dec-01 & 0,33 & 0,38 & 0,79 & 16,91 & 1,14 & $-4,91$ & $-2,76$ & yes \\
\hline & 8,09 & 60 & Jan-98 & Dec-02 & $-1,46$ & $-2,47$ & 0,87 & 23,99 & 1,04 & $-4,85$ & $-2,54$ & yes \\
\hline & 8,09 & 54 & Jan-99 & Dec-03 & $-1,25$ & $-3,15$ & 0,84 & 29,75 & 1,37 & $-6,14$ & $-3,42$ & yes \\
\hline & 8,10 & 60 & Jan-97 & Dec-01 & $-0,42$ & $-0,42$ & 0,86 & 16,15 & 0,98 & $-4,45$ & $-2,49$ & yes \\
\hline & 8,10 & 60 & Jan-98 & Dec-02 & $-1,61$ & $-2,47$ & 0,91 & 22,58 & 0,87 & $-4,32$ & $-2,30$ & yes \\
\hline & 8,10 & 54 & Jan-99 & Dec-03 & $-1,14$ & $-2,64$ & 0,85 & 27,81 & 1,17 & $-5,42$ & $-3,07$ & yes \\
\hline & 8,11 & 60 & Jan-97 & Dec-01 & 0,99 & 1,02 & 0,79 & 15,15 & 0,96 & $-4,49$ & $-2,61$ & yes \\
\hline & 8,11 & 60 & Jan-98 & Dec-02 & $-0,43$ & $-0,64$ & 0,86 & 20,74 & 0,94 & $-4,53$ & $-2,53$ & yes \\
\hline & 8,11 & 54 & Jan-99 & Dec-03 & $-0,26$ & $-0,54$ & 0,82 & 24,35 & 1,12 & $-5,67$ & & yes \\
\hline & 8,12 & 60 & Jan-97 & Dec-01 & 0,55 & 0,62 & 0,82 & 17,07 & 1,20 & $-5,29$ & $-3,13$ & yes \\
\hline & 8,12 & 50 & Jan-98 & Dec-02 & 0,75 & 0,85 & 0,80 & 15,60 & 1,17 & $-4,56$ & $-2,44$ & yes \\
\hline & 8,12 & 38 & Jan-99 & Dec-03 & 2,93 & 3,35 & 0,63 & 11,55 & 1,51 & $-5,93$ & & yes \\
\hline Slovak Republic & 8,01 & 50 & Jan-95 & Dec-99 & 3,59 & 2,62 & 0,58 & 6,85 & 0,18 & $-1,21$ & $-0,33$ & no \\
\hline & 8,01 & 56 & Jan-96 & Dec-00 & 3,49 & 2,91 & 0,58 & 7,63 & 0,19 & $-1,44$ & $-0,75$ & no \\
\hline & 8,01 & 56 & Jan-97 & Dec-01 & 3,11 & 3,21 & 0,62 & 9,73 & 0,24 & $-1,82$ & & no \\
\hline & 8,01 & 60 & Jan-98 & Dec-02 & $-0,02$ & $-0,03$ & 0,90 & 17,17 & 0,83 & $-4,00$ & $-2,09$ & yes \\
\hline & 8,01 & 60 & Jan-99 & Dec-03 & $-2,75$ & $-5,91$ & 1,18 & 24,09 & 1,19 & $-5,01$ & $-2,59$ & yes \\
\hline & 8,02 & 50 & Jan-95 & Dec-99 & 6,47 & 3,89 & 0,42 & 4,14 & 0,12 & $-1,24$ & & no \\
\hline & 8,02 & 56 & Jan-96 & Dec-00 & 6,03 & 4,09 & 0,45 & 4,77 & 0,13 & $-1,44$ & & no \\
\hline & 8,02 & 56 & Jan-97 & Dec-01 & 5,17 & 4,23 & 0,52 & 6,43 & 0,17 & $-2,05$ & & no \\
\hline & 8,02 & 60 & Jan-98 & Dec-02 & 0,89 & 1,05 & 0,89 & 13,38 & 0,69 & $-3,56$ & $-2,49$ & yes \\
\hline & 8,02 & 60 & Jan-99 & Dec-03 & $-3,43$ & $-5,19$ & 1,31 & 18,85 & 1,00 & $-4,43$ & $-2,91$ & yes \\
\hline & 8,03 & 50 & Jan-95 & Dec-99 & 8,85 & 6,39 & 0,23 & 2,67 & 0,09 & $-0,77$ & & no \\
\hline & 8,03 & 56 & Jan-96 & Dec-00 & 9,10 & 7,21 & 0,22 & 2,68 & 0,08 & $-0,75$ & $-1,06$ & no \\
\hline
\end{tabular}


Table B4: 5-year rolling symmetric cointegration of bank rate and money market ratı

\begin{tabular}{|c|c|c|c|c|c|c|c|c|c|c|c|c|}
\hline \multirow[b]{2}{*}{ country } & \multirow[b]{2}{*}{ rate } & \multirow{2}{*}{$\begin{array}{l}\text { obser- } \\
\text { vations }\end{array}$} & \multicolumn{2}{|c|}{ rolling sample } & \multicolumn{2}{|c|}{ intercept } & \multicolumn{2}{|c|}{ slope } & \multirow[b]{2}{*}{ DW } & \multirow[b]{2}{*}{ DF } & \multirow[b]{2}{*}{$\mathrm{ADF}$} & \multirow{2}{*}{$\begin{array}{l}\text { cointe- } \\
\text { gration? }\end{array}$} \\
\hline & & & from & to & coefficient & t-statistic & coefficient & t-statistic & & & & \\
\hline & 8,03 & 56 & Jan-97 & Dec-01 & 7,07 & 6,09 & 0,34 & 4,48 & 0,11 & $-1,48$ & & no \\
\hline & 8,03 & 60 & Jan-98 & Dec-02 & 2,93 & 3,27 & 0,69 & 9,77 & 0,38 & $-2,59$ & $-1,97$ & no \\
\hline & 8,03 & 60 & Jan-99 & Dec-03 & $-2,79$ & $-3,40$ & 1,26 & 14,65 & 0,58 & $-3,28$ & & yes \\
\hline & 8,04 & 50 & Jan-95 & Dec-99 & 11,83 & 20,08 & $-0,05$ & $-1,42$ & 0,09 & $-1,39$ & $-1,61$ & no \\
\hline & 8,04 & 56 & Jan-96 & Dec-00 & 9,65 & 18,02 & 0,06 & 1,83 & 0,09 & $-0,34$ & $-1,46$ & no \\
\hline & 8,04 & 56 & Jan-97 & Dec-01 & 7,44 & 11,96 & 0,18 & 4,44 & 0,10 & $-1,11$ & $-1,48$ & no \\
\hline & 8,04 & 60 & Jan-98 & Dec-02 & 4,97 & 7,73 & 0,36 & 7,05 & 0,22 & $-1,75$ & $-0,98$ & no \\
\hline & 8,04 & 60 & Jan-99 & Dec-03 & 0,62 & 0,94 & 0,80 & 11,61 & 0,41 & $-2,74$ & $-1,42$ & yes \\
\hline & 8,05 & 50 & Jan-95 & Dec-99 & 12,76 & 19,74 & $-0,12$ & $-2,96$ & 0,17 & $-3,14$ & $-2,60$ & no \\
\hline & 8,05 & 56 & Jan-96 & Dec-00 & 9,15 & 24,12 & 0,07 & 2,94 & 0,33 & $-2,02$ & & no \\
\hline & 8,05 & 56 & Jan-97 & Dec-01 & 6,99 & 15,26 & 0,18 & 6,04 & 0,25 & $-1,81$ & & no \\
\hline & 8,05 & 60 & Jan-98 & Dec-02 & 5,16 & 9,50 & 0,30 & 7,00 & 0,31 & $-1,54$ & $-0,61$ & no \\
\hline & 8,05 & 60 & Jan-99 & Dec-03 & 2,19 & 3,50 & 0,59 & 8,95 & 0,42 & $-2,67$ & & yes \\
\hline & 8,06 & 50 & Jan-95 & Dec-99 & 17,47 & 22,90 & $-0,25$ & $-5,43$ & 0,61 & $-3,55$ & $-4,13$ & yes \\
\hline & 8,06 & 56 & Jan-96 & Dec-00 & 14,47 & 34,23 & $-0,10$ & $-3,69$ & 0,87 & $-4,07$ & $-3,99$ & yes \\
\hline & 8,06 & 56 & Jan-97 & Dec-01 & 11,11 & 16,55 & 0,07 & 1,54 & 0,23 & $-1,35$ & & no \\
\hline & 8,06 & 60 & Jan-98 & Dec-02 & 8,72 & 10,43 & 0,19 & 2,94 & 0,15 & $-1,18$ & $-0,93$ & no \\
\hline & 8,06 & 60 & Jan-99 & Dec-03 & 3,51 & 3,51 & 0,70 & 6,70 & 0,16 & $-1,54$ & & no \\
\hline & 8,07 & 50 & Jan-95 & Dec-99 & 4,59 & 18,17 & 0,00 & 0,11 & 0,36 & $-2,43$ & & no \\
\hline & 8,07 & 56 & Jan-96 & Dec-00 & 5,08 & 31,85 & $-0,02$ & $-2,33$ & 0,14 & $-1,49$ & & no \\
\hline & 8,07 & 56 & Jan-97 & Dec-01 & 4,58 & 61,17 & 0,00 & $-0,47$ & 0,35 & $-0,92$ & $-0,97$ & no \\
\hline & 8,07 & 60 & Jan-98 & Dec-02 & 3,92 & 21,42 & 0,03 & 1,89 & 0,07 & $-0,06$ & & no \\
\hline & 8,07 & 60 & Jan-99 & Dec-03 & 6,96 & 9,62 & $-0,20$ & $-2,70$ & 0,15 & $-1,04$ & $-0,98$ & no \\
\hline
\end{tabular}

See notes to table B2. 


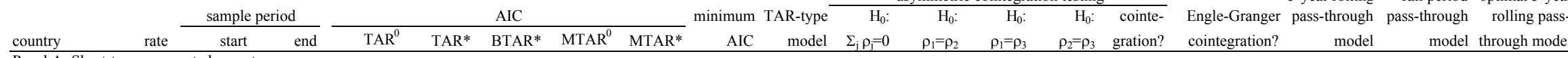

\begin{tabular}{|c|c|c|c|c|c|c|c|c|c|c|c|c|c|c|c|c|c|c|c|}
\hline \multicolumn{20}{|c|}{ Panel A: Short-term corporate loan rates } \\
\hline \multirow{4}{*}{$\begin{array}{l}\text { Czech Republic } \\
\text { Estonia }\end{array}$} & 4 & $\operatorname{Jan} 99$ & Dez 03 & $-42,95$ & $-43,54$ & $-46,68$ & $-44,21$ & $-47,19$ & $-47,19$ & MTAR* & 7,44 & 3,46 & & & yes,asym & yes & MTAR* & SYM & MTAR* \\
\hline & 4 & Jan 97 & Dez 01 & 246,01 & 244,67 & 243,98 & 245,02 & 243,77 & 243,77 & MTAR* & 3,83 & 2,39 & & & no & yes & SYM & SYM & SYM \\
\hline & 4 & Jan 98 & Dez 02 & 250,74 & 249,04 & 243,09 & 249,74 & 246,44 & 243,09 & BTAR* & 6,29 & 8,18 & 0,01 & 7,47 & yes,asym & yes & BTAR* & SYM & SYM \\
\hline & 4 & Jan 99 & Dez 03 & 217,77 & 216,34 & 215,20 & 217,45 & 214,73 & 214,73 & MTAR* & 3,23 & 2,78 & & & no & yes & SYM & SYM & SYM \\
\hline \multirow[t]{4}{*}{ Hungary } & 4 & Jan 95 & Dez 99 & 150,53 & 145,87 & 144,53 & 150,30 & 143,71 & 143,71 & MTAR* & 4,97 & 6,47 & & & no & no & STD & MTAR* & MTAR* \\
\hline & 4 & Jan 96 & Dez 00 & 115,81 & 113,91 & 107,89 & 117,26 & 112,92 & 107,89 & BTAR* & 6,07 & 9,86 & 2,85 & 8,05 & yes,asym & yes & BTAR* & MTAR* & MTAR* \\
\hline & 4 & Jan 98 & Dez 02 & 80,42 & 79,30 & 79,48 & 70,89 & 58,94 & 58,94 & MTAR* & 25,99 & 23,57 & & & yes,asym & yes & MTAR* & MTAR* & MTAR* \\
\hline & 4 & Jan 99 & Dez 03 & 80,54 & 79,63 & 77,76 & 80,07 & 79,52 & 77,76 & BTAR* & 12,05 & 4,46 & 0,30 & 4,38 & yes,asym & yes & BTAR* & MTAR* & MTAR* \\
\hline Latvia & 4 & Jan 99 & Dez 03 & 280,51 & 276,17 & 268,41 & 279,03 & 275,66 & 268,41 & BTAR* & 7,27 & 15,11 & 4,39 & 10,19 & yes,asym & no & BTAR* & STD & BTAR* \\
\hline Lithuania & 4 & Jan 99 & Dez 03 & 288,10 & 280,75 & 279,34 & 283,91 & 275,72 & 275,72 & MTAR* & 9,85 & 13,67 & & & yes,asym & yes & MTAR* & MTAR* & MTAR* \\
\hline \multirow[t]{3}{*}{ Poland } & 4 & Jan 96 & Dez 00 & 202,31 & 201,36 & 197,36 & 201,98 & 200,67 & 197,36 & BTAR* & 3,86 & 6,03 & 0,20 & 6,37 & no & yes & SYM & SYM & SYM \\
\hline & 4 & Jan 97 & Dez 01 & 258,32 & 257,06 & 247,85 & 256,87 & 256,35 & 247,85 & BTAR* & 6,07 & 12,12 & 0,13 & 12,22 & yes,asym & yes & BTAR* & SYM & SYM \\
\hline & 4 & Jan 99 & Dez 03 & 187,70 & 182,99 & 184,52 & 188,56 & 187,68 & 182,99 & TAR* & 5,86 & 7,29 & & & no & yes & SYM & SYM & SYM \\
\hline \multirow[t]{5}{*}{ Slovak Republic } & 4 & Jan 95 & Dez 99 & 174,59 & 173,33 & 171,49 & 174,32 & 168,02 & 168,02 & MTAR* & 3,40 & 6,21 & & & no & no & STD & STD & STD \\
\hline & 4 & Jan 96 & Dez 00 & 219,00 & 218,22 & 208,07 & 219,28 & 210,38 & 208,07 & BTAR* & 4,63 & 12,97 & 0,49 & 12,02 & no & no & STD & STD & STD \\
\hline & 4 & Jan 97 & Dez 01 & 221,59 & 220,76 & 220,08 & 221,00 & 216,92 & 216,92 & MTAR* & 2,72 & 4,88 & & & no & no & STD & STD & STD \\
\hline & 4 & Jan 98 & Dez 02 & 288,04 & 287,27 & 280,85 & 287,56 & 283,35 & 280,85 & BTAR* & 3,42 & 8,76 & 0,20 & 8,11 & no & yes & SYM & STD & STD \\
\hline & 4 & $\operatorname{Jan} 99$ & Dez 03 & 220,58 & 210,75 & 208,28 & 219,31 & 209,61 & 208,28 & BTAR* & 7,54 & 6,25 & 18,44 & 4,06 & yes,asym & yes & BTAR* & STD & STD \\
\hline \multicolumn{20}{|c|}{ Panel B: Long-term corpora } \\
\hline \multirow{3}{*}{ Estonia } & 5 & Jan 97 & Dez 01 & 237,15 & 233,72 & 234,46 & 234,06 & 231,76 & 231,76 & MTAR* & 8,52 & 5,63 & & & yes,asym & yes & MTAR* & SYM & $\overline{\text { SYM }}$ \\
\hline & 5 & Jan 98 & Dez 02 & 235,90 & 227,89 & 229,51 & 238,44 & 231,39 & 227,89 & TAR* & 13,40 & 13,89 & & & yes,asym & yes & TAR* & SYM & SYM \\
\hline & 5 & Jan 99 & Dez 03 & 220,41 & 217,42 & 214,48 & 220,37 & 218,39 & 214,48 & BTAR* & 3,10 & 4,50 & 0,33 & 7,14 & no & yes & SYM & SYM & SYM \\
\hline \multirow[t]{4}{*}{ Hungary } & 5 & Jan 95 & Dez 99 & 197,24 & 196,15 & 196,90 & 195,93 & 195,06 & 195,06 & MTAR* & 3,35 & 1,99 & & & no & yes & SYM & SYM & MTAR* \\
\hline & 5 & Jan 96 & Dez 00 & 174,62 & 172,56 & 174,34 & 169,57 & 168,67 & 168,67 & MTAR* & 7,54 & 7,83 & & & yes,asym & yes & MTAR* & SYM & MTAR* \\
\hline & 5 & Jan 98 & Dez 02 & 124,21 & 123,22 & 124,14 & 123,93 & 122,22 & 122,22 & MTAR* & 5,32 & 2,03 & & & no & yes & SYM & SYM & MTAR* \\
\hline & 5 & Jan 99 & Dez 03 & 176,43 & 171,58 & 170,85 & 174,78 & 168,84 & 168,84 & MTAR* & 13,22 & 7,74 & & & yes,asym & yes & MTAR* & SYM & MTAR* \\
\hline Latvia & 5 & Jan 99 & Dez 03 & 226,70 & 224,23 & 224,78 & 227,42 & 218,35 & 218,35 & MTAR* & 8,81 & 10,55 & & & yes,asym & yes & MTAR* & SYM & MTAR* \\
\hline \multirow[t]{2}{*}{ Lithuania } & 5,01 & Jan 99 & Dez 03 & 278,38 & 277,59 & 275,47 & 278,05 & 275,08 & 275,08 & MTAR* & 4,15 & 3,10 & & & no & yes & SYM & SYM & SYM \\
\hline & 5,02 & Jan 99 & Dez 03 & 257,02 & 249,48 & 244,37 & 250,17 & 249,86 & 244,37 & BTAR* & 5,51 & 6,69 & 2,09 & 13,82 & no & yes & SYM & SYM & SYM \\
\hline \multirow[t]{5}{*}{ Poland } & 5,01 & Jan 97 & Dez 01 & 259,27 & 257,12 & 249,45 & 256,79 & 255,53 & 249,45 & BTAR* & 5,45 & 11,34 & 0,47 & 11,51 & no & yes & SYM & SYM & SYM \\
\hline & 5,01 & Jan 98 & Dez 02 & 226,38 & 224,72 & 222,80 & 222,02 & 220,46 & 220,46 & MTAR* & 4,67 & 5,69 & & & no & yes & SYM & SYM & SYM \\
\hline & 5,01 & Jan 99 & Dez 03 & 187,23 & 183,14 & 185,10 & 190,84 & 189,50 & 183,14 & TAR* & 6,42 & 8,19 & & & no & yes & SYM & SYM & SYM \\
\hline & 5,02 & Jan 98 & Dez 02 & 220,31 & 218,81 & 208,22 & 219,36 & 214,52 & 208,22 & BTAR* & 6,22 & 14,01 & 0,04 & 14,05 & yes,asym & yes & BTAR* & SYM & SYM \\
\hline & 5,02 & Jan 99 & Dez 03 & 197,32 & 190,66 & 192,33 & 200,62 & 197,57 & 190,66 & TAR* & 7,19 & 10,75 & & & yes,asym & yes & TAR* & SYM & SYM \\
\hline \multicolumn{20}{|c|}{ Panel C: Consumer loan rat } \\
\hline Czech Republic & 3 & Jan 93 & Dez 97 & 247,68 & 245,52 & 243,30 & 247,88 & 246,05 & 243,30 & BTAR* & 5,80 & 6,13 & 0,25 & 3,93 & no & yes & SYM & SYM & MTAR* \\
\hline & 3 & Jan 94 & Dez 98 & 220,32 & 218,71 & 220,03 & 217,33 & 205,51 & 205,51 & MTAR* & 8,60 & 15,33 & & & yes,asym & yes & MTAR* & SYM & MTAR* \\
\hline & 3 & Jan 95 & Dez 99 & 158,83 & 158,31 & 155,86 & 162,39 & 160,21 & 155,86 & BTAR* & 3,06 & 5,03 & 3,61 & 4,08 & no & no & STD & SYM & MTAR* \\
\hline & 3 & Jan 96 & Dez 00 & 167,32 & 164,89 & 166,05 & 165,12 & 158,11 & 158,11 & MTAR* & 10,37 & 13,32 & & & yes,asym & yes & MTAR* & SYM & MTAR* \\
\hline & 3 & Jan 97 & Dez 01 & 133,04 & 131,75 & 131,53 & 133,04 & 123,26 & 123,26 & MTAR* & 7,79 & 9,90 & & & yes,asym & yes & MTAR* & SYM & MTAR* \\
\hline & 3 & Jan 98 & Dez 02 & 93,60 & 91,32 & 83,29 & 92,87 & 92,67 & 83,29 & BTAR* & 6,94 & 11,98 & 1,16 & 12,03 & yes,asym & yes & BTAR* & SYM & MTAR* \\
\hline & 3 & Jan 99 & Dez 03 & 110,63 & 106,02 & 106,48 & 110,67 & 105,24 & 105,24 & MTAR* & 8,62 & 5,29 & & & yes,asym & yes & MTAR* & SYM & MTAR* \\
\hline Estonia & 3 & Jan 97 & Dez 01 & 257,21 & 256,83 & 257,83 & 251,75 & 248,23 & 248,23 & MTAR* & 10,53 & 8,79 & & & yes,asym & yes & MTAR* & MTAR* & MTAR* \\
\hline & 3 & Jan 98 & Dez 02 & 267,59 & 265,77 & 267,93 & 266,66 & 261,62 & 261,62 & MTAR* & 13,10 & 5,78 & & & yes,asym & yes & MTAR* & MTAR* & MTAR* \\
\hline & 3 & Jan 99 & Dez 03 & 243,02 & 237,72 & 237,74 & 240,89 & 237,74 & 237,72 & $\mathrm{TAR}^{*}$ & 13,40 & 6,31 & & & yes,asym & yes & $\mathrm{TAR}^{*}$ & MTAR* & MTAR* \\
\hline Hungary & 3 & Jan 95 & Dez 99 & 143,22 & 135,83 & 134,50 & 143,32 & 138,25 & 134,50 & BTAR* & 5,99 & 10,44 & 1,29 & 3,00 & yes,asym & yes & BTAR* & STD & SYM \\
\hline & 3 & Jan 96 & Dez 00 & 133,96 & 133,30 & 126,89 & 134,39 & 130,06 & 126,89 & BTAR* & 5,51 & 8,56 & 0,68 & 8,64 & no & yes & SYM & STD & SYM \\
\hline & 3 & Jan 97 & Dez 01 & 153,98 & 152,45 & 152,83 & 155,40 & 151,85 & 151,85 & MTAR* & 2,13 & 3,31 & & & no & yes & SYM & STD & SYM \\
\hline & 3 & Jan 98 & Dez 02 & 160,42 & 159,38 & 156,91 & 160,68 & 159,43 & 156,91 & BTAR* & 3,06 & 4,66 & 0,41 & 5,08 & no & yes & SYM & STD & SYM \\
\hline
\end{tabular}




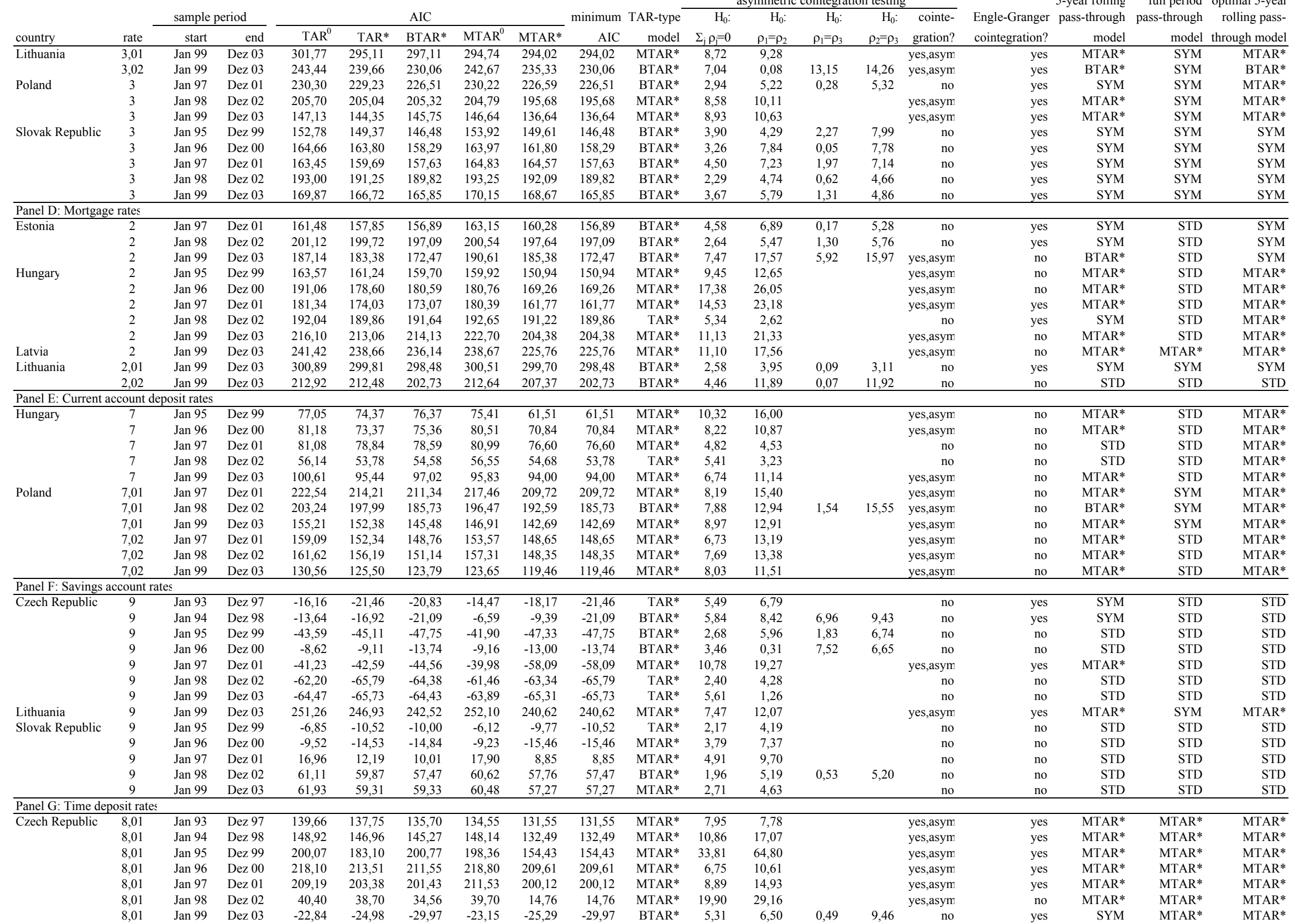


AIC

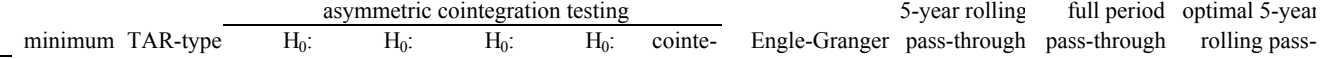

\begin{tabular}{|c|c|c|c|c|c|c|c|c|c|c|c|c|c|c|c|c|c|c|c|}
\hline \multirow[b]{2}{*}{ country } & \multirow[b]{2}{*}{ rate } & \multicolumn{2}{|c|}{ sample period } & \multicolumn{5}{|c|}{ AIC } & \multirow{2}{*}{$\begin{array}{r}\text { minimum } \\
\text { AIC }\end{array}$} & \multirow{2}{*}{$\begin{array}{r}\text { TAR-type } \\
\text { model }\end{array}$} & \multirow{2}{*}{$\begin{array}{r}\mathrm{H}_{0}: \\
\sum_{\mathrm{i} \rho}=0\end{array}$} & \multirow{2}{*}{$\begin{array}{r}H_{0}: \\
\rho_{1}=\rho_{2}\end{array}$} & \multirow{2}{*}{$\begin{array}{r}H_{0}: \\
\rho_{1}=\rho_{3}\end{array}$} & \multirow{2}{*}{$\begin{array}{r}H_{0}: \\
\rho_{2}=\rho_{3}\end{array}$} & \multirow{2}{*}{$\begin{array}{l}\text { cointe- } \\
\text { gration? }\end{array}$} & & pass-through & pass-through & rolling pass- \\
\hline & & start & end & $\mathrm{TAR}^{0}$ & TAR* & BTAR* & MTAR $^{0}$ & MTAR* & & & & & & & & cointegration? & model & model & through model \\
\hline & 8,02 & Jan 93 & Dez 97 & $\begin{array}{ll}105,07 \\
\end{array}$ & 100,53 & 101,67 & 102,69 & 96,96 & 96,96 & MTAR* & 5,95 & 8,14 & & & no & no & STD & BTAR* & BTAR* $^{*}$ \\
\hline & 8,02 & Jan 94 & Dez 98 & 86,93 & 86,37 & 74,50 & 88,02 & 84,72 & 74,50 & BTAR* & 7,10 & 14,40 & 1,27 & 14,30 & yes,asym & no & BTAR* & BTAR* & BTAR* \\
\hline & 8,02 & Jan 95 & Dez 99 & 204,62 & 201,11 & 200,11 & 200,12 & 198,98 & 198,98 & MTAR* & 3,74 & 5,35 & & & no & no & STD & BTAR* & BTAR* \\
\hline & 8,02 & $\operatorname{Jan} 96$ & Dez 00 & 237,78 & 235,14 & 235,29 & 237,40 & 233,38 & 233,38 & MTAR* & 3,64 & 4,11 & & & no & yes & SYM & BTAR* & BTAR* \\
\hline & 8,02 & Jan 97 & Dez 01 & 182,65 & 171,72 & 162,68 & 183,22 & 167,71 & 162,68 & BTAR* & 11,67 & 5,48 & 9,41 & 29,81 & yes,asym & yes & BTAR* & BTAR* & BTAR* \\
\hline & 8,02 & $\operatorname{Jan} 98$ & Dez 02 & 142,32 & 138,33 & 138,89 & 143,52 & 137,66 & 137,66 & MTAR* & 6,44 & 5,61 & & & yes,asym & yes & MTAR* & BTAR* & BTAR* \\
\hline & 8,02 & Jan 99 & Dez 03 & 31,80 & 30,04 & 30,90 & 32,73 & 28,98 & 28,98 & MTAR* & 5,59 & 3,70 & & & no & yes & SYM & BTAR* & BTAR* \\
\hline & 8,03 & $\operatorname{Jan} 93$ & Dez 97 & 237,41 & 236,27 & 236,12 & 239,61 & 235,84 & 235,84 & MTAR* & 3,20 & 3,54 & & & no & no & STD & STD & STD \\
\hline & 8,03 & Jan 94 & Dez 98 & 253,21 & 252,26 & 249,41 & 253,86 & 251,55 & 249,41 & BTAR* & 2,35 & 3,82 & 0,17 & 4,47 & no & no & STD & STD & STD \\
\hline & 8,03 & Jan 95 & Dez 99 & 54,30 & 54,09 & 47,61 & 57,20 & 48,02 & 47,61 & BTAR* & 4,95 & 8,24 & 4,60 & 8,21 & no & no & STD & STD & STD \\
\hline & 8,03 & Jan 96 & Dez 00 & 112,97 & 106,54 & 98,08 & 112,64 & 105,17 & 98,08 & BTAR* & 8,31 & 17,22 & 0,70 & 17,70 & yes,asym & yes & BTAR* & STD & STD \\
\hline & 8,03 & Jan 97 & Dez 01 & 56,30 & 48,69 & 5,46 & 57,50 & 36,00 & 5,46 & BTAR* & 30,79 & 38,74 & 12,10 & 81,85 & yes,asym & yes & BTAR* & STD & STD \\
\hline & 8,03 & Jan 98 & Dez 02 & $-106,93$ & $-109,32$ & $-110,69$ & $-107,32$ & $-112,74$ & $-112,74$ & MTAR* & 4,46 & 5,61 & & & no & no & STD & STD & STD \\
\hline & 8,03 & Jan 99 & Dez 03 & $-147,89$ & $-149,14$ & $-148,37$ & $-150,06$ & $-155,21$ & $-155,21$ & MTAR* & 5,34 & 7,69 & & & no & no & STD & STD & STD \\
\hline & 8,04 & $\operatorname{Jan} 93$ & Dez 97 & 145,75 & 141,00 & 145,24 & 145,52 & 141,70 & 141,00 & TAR* & 3,68 & 4,44 & & & no & yes & SYM & MTAR* & MTAR* \\
\hline & 8,04 & Jan 94 & Dez 98 & 142,20 & 138,79 & 140,91 & 142,81 & 133,79 & 133,79 & MTAR* & 6,68 & 8,75 & & & yes,asym & yes & MTAR* & MTAR* & MTAR* \\
\hline & 8,04 & Jan 95 & Dez 99 & 184,80 & 170,83 & 184,36 & 184,12 & 130,52 & 130,52 & MTAR* & 42,58 & 82,48 & & & yes,asym & no & MTAR* & MTAR* & MTAR* \\
\hline & 8,04 & Jan 96 & Dez 00 & 210,46 & 206,04 & 207,50 & 209,68 & 201,93 & 201,93 & MTAR* & 6,26 & 8,92 & & & yes,asym & yes & MTAR* & MTAR* & MTAR* \\
\hline & 8,04 & Jan 97 & Dez 01 & 188,96 & 182,22 & 179,38 & 191,32 & 178,15 & 178,15 & MTAR* & 9,93 & 16,90 & & & yes,asym & yes & MTAR* & MTAR* & MTAR* \\
\hline & 8,04 & Jan 98 & Dez 02 & 46,64 & 42,47 & 41,26 & 45,16 & 9,61 & 9,61 & MTAR* & 30,09 & 47,17 & & & yes,asym & no & MTAR* & MTAR* & MTAR* \\
\hline & 8,04 & Jan 99 & Dez 03 & $-38,18$ & $-39,42$ & $-44,99$ & $-40,21$ & $-41,41$ & $-44,99$ & BTAR* & 4,82 & 8,36 & 1,27 & 8,11 & no & yes & SYM & MTAR* & MTAR* \\
\hline Estonia & 8,01 & Jan 96 & Dez 00 & 231,96 & 230,08 & 225,42 & 232,12 & 228,53 & 225,42 & BTAR* & 5,51 & 8,27 & 0,32 & 7,29 & no & yes & SYM & SYM & SYM \\
\hline & 8,01 & Jan 97 & Dez 01 & 219,75 & 219,08 & 215,60 & 219,23 & 213,10 & 213,10 & MTAR* & 8,04 & 6,32 & & & yes,asym & yes & MTAR* & SYM & SYM \\
\hline & 8,01 & $\operatorname{Jan} 98$ & Dez 02 & 166,72 & 163,31 & 161,14 & 168,16 & 160,92 & 160,92 & MTAR* & 11,60 & 6,90 & & & yes,asym & yes & MTAR* & SYM & SYM \\
\hline & 8,01 & Jan 99 & Dez 03 & 114,63 & 105,86 & 100,24 & 111,97 & 111,13 & 100,24 & BTAR* & 7,65 & 15,68 & 0,04 & 7,14 & yes,asym & yes & BTAR* & SYM & SYM \\
\hline & 8,02 & $\operatorname{Jan} 96$ & Dez 00 & 160,30 & 159,14 & 157,88 & 161,66 & 161,10 & 157,88 & BTAR* & 2,62 & 3,75 & 2,19 & 4,66 & no & no & STD & STD & STD \\
\hline & 8,02 & Jan 97 & Dez 01 & 169,95 & 168,10 & 165,01 & 170,46 & 167,41 & 165,01 & BTAR* & 4,15 & 3,91 & 1,47 & 5,14 & no & no & STD & STD & STD \\
\hline & 8,02 & $\operatorname{Jan} 98$ & Dez 02 & 149,73 & 145,90 & 138,57 & 149,55 & 143,32 & 138,57 & BTAR* & 10,27 & 0,49 & 7,99 & 13,00 & yes,asym & yes & BTAR* & STD & STD \\
\hline & 8,02 & Jan 99 & Dez 03 & 15,44 & 14,20 & 14,74 & 12,06 & 9,40 & 9,40 & MTAR* & 7,59 & 7,67 & & & yes,asym & yes & MTAR* & STD & STD \\
\hline & 8,03 & Jan 96 & Dez 00 & 223,09 & 222,59 & 220,98 & 222,77 & 221,54 & 220,98 & BTAR* & 3,72 & 3,81 & 0,07 & 3,26 & no & yes & SYM & SYM & SYM \\
\hline & 8,03 & Jan 97 & Dez 01 & 204,23 & 203,68 & 202,79 & 202,98 & 201,54 & 201,54 & MTAR* & 5,21 & 2,46 & & & no & no & STD & SYM & SYM \\
\hline & 8,03 & $\operatorname{Jan} 98$ & Dez 02 & 119,35 & 113,41 & 106,25 & 119,54 & 117,62 & 106,25 & BTAR* & 11,13 & 13,83 & 2,25 & 12,01 & yes,asym & yes & BTAR* & SYM & SYM \\
\hline & 8,03 & Jan 99 & Dez 03 & 74,89 & 69,81 & 71,24 & 73,83 & 72,58 & 69,81 & TAR* & 3,56 & 4,82 & & & no & yes & SYM & SYM & SYM \\
\hline & 8,04 & Jan 97 & Dez 01 & 151,81 & 151,01 & 143,27 & 151,87 & 146,19 & 143,27 & BTAR* & 5,80 & 0,12 & 9,33 & 9,94 & no & yes & SYM & MTAR* & SYM \\
\hline & 8,04 & $\operatorname{Jan} 98$ & Dez 02 & 134,99 & 129,20 & 128,02 & 134,74 & 127,76 & 127,76 & MTAR* & 13,05 & 7,13 & & & yes,asym & yes & MTAR* & MTAR* & SYM \\
\hline & 8,04 & Jan 99 & Dez 03 & 6,53 & 5,77 & 4,17 & 7,27 & 1,16 & 1,16 & MTAR* & 5,96 & 6,65 & & & no & yes & SYM & MTAR* & SYM \\
\hline & 8,05 & Jan 96 & Dez 00 & 191,77 & 188,26 & 181,36 & 193,34 & 188,19 & 181,36 & BTAR* & 5,97 & 10,82 & 2,33 & 12,36 & no & no & STD & MTAR* & MTAR* \\
\hline & 8,05 & Jan 97 & Dez 01 & 194,21 & 191,91 & 190,43 & 194,73 & 180,47 & 180,47 & MTAR* & 11,79 & 14,93 & & & yes,asym & no & MTAR* & MTAR* & MTAR* \\
\hline & 8,05 & Jan 98 & Dez 02 & 173,05 & 171,03 & 156,15 & 172,80 & 158,52 & 156,15 & BTAR* & 11,86 & 11,46 & 9,18 & 18,71 & yes,asym & yes & BTAR* & MTAR* & MTAR* \\
\hline & 8,05 & Jan 99 & Dez 03 & 45,19 & 44,48 & 38,64 & 42,88 & 34,72 & 34,72 & MTAR* & 9,27 & 11,61 & & & yes,asym & yes & MTAR* & MTAR* & MTAR* \\
\hline & 8,06 & Jan 96 & Dez 00 & 270,22 & 257,21 & 259,19 & 262,37 & 258,82 & 257,21 & TAR* & 9,87 & 13,46 & & & yes,asym & yes & TAR* & MTAR* & MTAR* \\
\hline & 8,06 & Jan 97 & Dez 01 & 216,73 & 214,66 & 216,66 & 217,15 & 214,10 & 214,10 & MTAR* & 2,95 & 2,93 & & & no & yes & SYM & MTAR* & MTAR* \\
\hline & 8,06 & Jan 98 & Dez 02 & 211,31 & 207,02 & 200,83 & 212,33 & 209,31 & 200,83 & BTAR* & 6,25 & 9,39 & 0,19 & 7,70 & yes,asym & no & BTAR* & MTAR* & MTAR* \\
\hline & 8,06 & $\operatorname{Jan} 99$ & Dez 03 & 167,31 & 161,79 & 160,31 & 168,40 & 163,22 & 160,31 & BTAR* & 4,04 & 8,77 & 2,49 & 7,16 & no & no & STD & MTAR* & MTAR* \\
\hline Hungary & 8,01 & Jan 95 & Dez 99 & 126,90 & 126,75 & 125,85 & 124,23 & 120,80 & 120,80 & MTAR* & 12,01 & 7,05 & & & yes,asym & yes & MTAR* & MTAR* & MTAR* \\
\hline & 8,01 & Jan 96 & Dez 00 & 98,86 & 97,42 & 97,75 & 94,45 & 93,35 & 93,35 & MTAR* & 9,99 & 5,45 & & & yes,asym & yes & MTAR* & MTAR* & MTAR* \\
\hline & 8,01 & Jan 97 & Dez 01 & 71, & 70,36 & 68,78 & 66,87 & 65,05 & 65,05 & MTAR* & 8,83 & 6,06 & & & yes,asym & yes & MTAR* & MTAR* & MTAR* \\
\hline & 8,01 & Jan 98 & Dez 02 & 78,57 & 75,29 & 74,64 & 73,21 & 72,14 & 72,14 & MTAR* & 9,49 & 6,27 & & & yes,asym & yes & MTAR* & MTAR* & MTAR* \\
\hline & 8,01 & Jan 99 & Dez 03 & 98,22 & 95,40 & 96,73 & 99,06 & 97,44 & 95,40 & TAR* & 13,15 & 5,18 & & & yes,asym & yes & TAR* & MTAR* & MTAR* \\
\hline & 8,02 & $\operatorname{Jan} 95$ & Dez 99 & 174,70 & 170,81 & 171,60 & 172,56 & 171,23 & 170,81 & TAR* & 6,42 & 4,25 & & & no & yes & SYM & SYM & SYM \\
\hline & 8,02 & $\operatorname{Jan} 96$ & Dez 00 & 154,43 & 153,82 & 150,31 & 154,04 & 152,05 & 150,31 & BTAR* & 4,46 & 5,64 & 0,00 & 5,64 & no & yes & SYM & SYM & SYM \\
\hline & 8,02 & Jan 97 & Dez 01 & 130,90 & 127,20 & 128,94 & 124,33 & 118,40 & 118,40 & MTAR* & 14,07 & 13,13 & & & yes,asym & yes & MTAR* & SYM & SYM \\
\hline & 8,02 & Jan 98 & Dez 02 & 116,04 & 115,61 & 113,79 & 114,96 & 110,46 & 110,46 & MTAR* & 9,73 & 5,31 & & & yes,asym & yes & MTAR* & SYM & SYM \\
\hline & 8,02 & Jan 99 & Dez 03 & 142,46 & 139,67 & $\begin{array}{l}140,78 \\
\end{array}$ & 144,59 & 142,38 & 139,67 & TAR* & 3,86 & 4,61 & & & no & yes & SYM & SYM & SYM \\
\hline
\end{tabular}


AIC

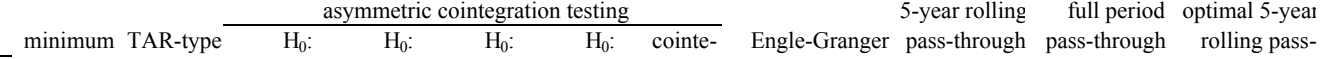

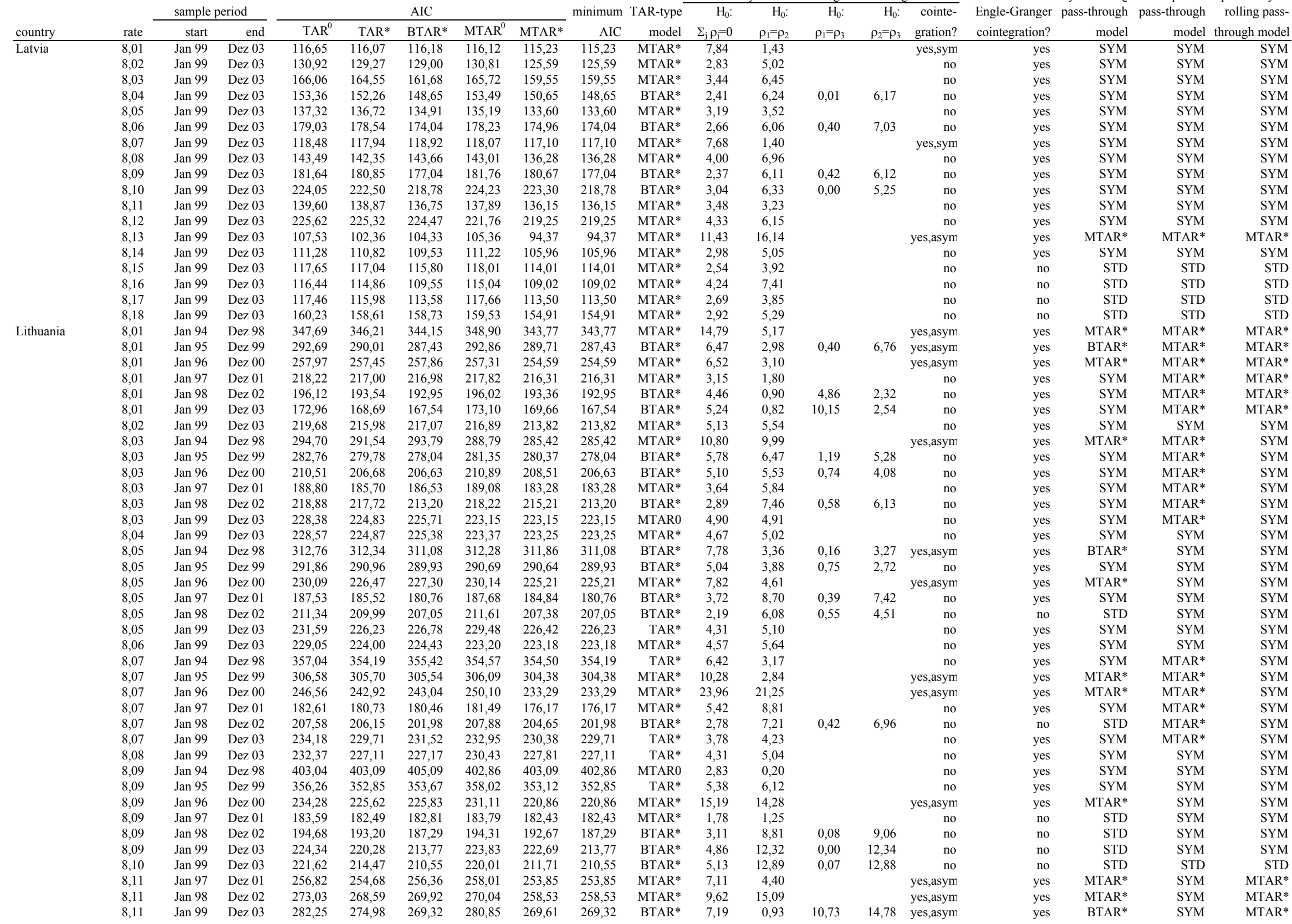




\begin{tabular}{|c|c|c|c|c|c|c|c|c|c|c|c|c|c|c|c|c|c|c|c|}
\hline \multirow[b]{2}{*}{ country } & \multirow[b]{2}{*}{ rate } & \multicolumn{2}{|c|}{ sample period } & \multicolumn{5}{|c|}{$\mathrm{AIC}$} & \multirow{2}{*}{$\begin{array}{r}\text { minimum } \\
\text { AIC }\end{array}$} & \multirow{2}{*}{$\begin{array}{r}\text { TAR-type } \\
\text { model }\end{array}$} & $\mathrm{H}_{0}:$ & $\mathrm{H}_{0}:$ & $\mathrm{H}_{0}:$ & $\mathrm{H}_{0}:$ & cointe- & \multirow{2}{*}{$\begin{array}{l}\text { Engle-Granger } \\
\text { cointegration? }\end{array}$} & \multirow{2}{*}{$\begin{array}{r}\text { pass-through } \\
\text { model }\end{array}$} & \multirow{2}{*}{$\begin{array}{r}\text { pass-through } \\
\text { model }\end{array}$} & rolling pass- \\
\hline & & start & end & $\mathrm{TAR}^{0}$ & TAR* & BTAR* & MTAR $^{0}$ & MTAR* & & & $\Sigma_{\mathrm{j}} \rho_{=}=0$ & $\rho_{1}=\rho_{2}$ & $\rho_{1}=\rho_{3}$ & $\rho_{2}=\rho_{3}$ & gration? & & & & through model \\
\hline & 8,12 & $\operatorname{Jan} 99$ & Dez 03 & 229,09 & 223,84 & 223,77 & 226,01 & 224,66 & 223,77 & BTAR* & 3,12 & 1,84 & 1,42 & 6,83 & no & no & STD & STD & STD \\
\hline & 8,13 & Jan 94 & Dez 98 & 288,50 & 287,15 & 283,59 & 288,56 & 283,15 & 283,15 & MTAR* & 10,18 & 6,34 & & & yes,asym & yes & MTAR* & MTAR* & MTAR* \\
\hline & 8,13 & $\operatorname{Jan} 95$ & Dez 99 & 282,05 & 280,33 & 281,06 & 281,49 & 275,10 & 275,10 & MTAR* & 10,34 & 7,84 & & & yes,asym & yes & MTAR* & MTAR* & MTAR* \\
\hline & 8,13 & Jan 96 & Dez 00 & 235,75 & 234,51 & 234,83 & 235,69 & 232,10 & 232,10 & MTAR* & 6,12 & 3,37 & & & yes,asym & yes & MTAR* & MTAR* & MTAR* \\
\hline & 8,13 & Jan 98 & Dez 02 & 210,90 & 209,61 & 206,22 & 210,73 & 206,42 & 206,22 & BTAR* & 3,63 & 6,20 & 0,25 & 6,19 & no & yes & SYM & MTAR* & MTAR* \\
\hline & 8,13 & Jan 99 & Dez 03 & 194,18 & 189,20 & 183,81 & 192,39 & 188,56 & 183,81 & BTAR* & 6,16 & 1,96 & 13,20 & 6,90 & yes,asym & yes & BTAR* & MTAR* & MTAR* \\
\hline & 8,14 & Jan 99 & Dez 03 & 224,66 & 220,00 & 220,86 & 219,40 & 219,40 & 219,40 & MTAR0 & 4,29 & 4,99 & & & no & yes & SYM & SYM & SYM \\
\hline & 8,15 & Jan 94 & Dez 98 & 195,13 & 187,43 & 186,85 & 187,34 & 183,19 & 183,19 & MTAR* & 12,05 & 16,35 & & & yes,asym & yes & MTAR* & MTAR* & MTAR* \\
\hline & 8,15 & $\operatorname{Jan} 95$ & Dez 99 & 176,68 & 176,21 & 175,98 & 176,85 & 171,35 & 171,35 & MTAR* & 10,17 & 5,17 & & & yes,asym & yes & MTAR* & MTAR* & MTAR* \\
\hline & 8,15 & Jan 96 & Dez 00 & 150,20 & 149,59 & 150,18 & 147,54 & 147,33 & 147,33 & MTAR* & 7,09 & 2,74 & & & yes,asym & yes & MTAR* & MTAR* & MTAR* \\
\hline & 8,15 & $\operatorname{Jan} 98$ & Dez 02 & 111,91 & 110,07 & 111,20 & 111,31 & 108,82 & 108,82 & MTAR* & 2,81 & 3,10 & & & no & yes & SYM & MTAR* & MTAR* \\
\hline & 8,15 & Jan 99 & Dez 03 & 96,33 & 95,19 & 92,76 & 94,69 & 92,49 & 92,49 & MTAR* & 4,96 & 3,72 & & & no & yes & SYM & MTAR* & MTAR* \\
\hline \multirow[t]{30}{*}{ Poland } & 8,01 & Jan 97 & Dez 01 & 230,81 & 229,47 & 229,13 & 230,39 & 226,62 & 226,62 & MTAR* & 4,49 & 3,99 & & & no & yes & SYM & SYM & SYM \\
\hline & 8,01 & Jan 98 & Dez 02 & 201,20 & 199,06 & 192,58 & 198,32 & 196,12 & 192,58 & BTAR* & 5,15 & 10,22 & 1,31 & 10,22 & no & yes & SYM & SYM & SYM \\
\hline & 8,01 & Jan 99 & Dez 03 & 164,21 & 161,27 & 158,56 & 160,45 & 159,38 & 158,56 & BTAR* & 4,74 & 8,05 & 2,45 & 5,72 & no & yes & SYM & SYM & SYM \\
\hline & 8,02 & Jan 97 & Dez 01 & 237,83 & 237,03 & 238,05 & 237,61 & 233,73 & 233,73 & MTAR* & 4,76 & 3,91 & & & no & yes & SYM & SYM & SYM \\
\hline & 8,02 & Jan 98 & Dez 02 & 210,91 & 209,02 & 202,47 & 208,26 & 207,81 & 202,47 & BTAR* & 5,41 & 9,99 & 1,25 & 10,03 & no & yes & SYM & SYM & SYM \\
\hline & 8,02 & Jan 99 & Dez 03 & 170,54 & 167,06 & 164,38 & 165,86 & 165,50 & 164,38 & BTAR* & 4,25 & 8,98 & 1,53 & 4,21 & no & yes & SYM & SYM & SYM \\
\hline & 8,03 & Jan 97 & Dez 01 & 243,24 & 241,87 & 236,04 & 242,54 & 240,79 & 236,04 & BTAR* & 4,92 & 8,01 & 1,13 & 8,88 & no & yes & SYM & SYM & SYM \\
\hline & 8,03 & $\operatorname{Jan} 98$ & Dez 02 & 212,87 & 210,76 & 203,99 & 209,47 & 206,91 & 203,99 & BTAR* & 5,52 & 10,50 & 1,45 & 10,37 & no & yes & SYM & SYM & SYM \\
\hline & 8,03 & Jan 99 & Dez 03 & 171,11 & 165,63 & 162,57 & 164,31 & 163,72 & 162,57 & BTAR* & 5,56 & 12,11 & 2,56 & 4,56 & no & yes & SYM & SYM & SY \\
\hline & 8,04 & Jan 97 & Dez 01 & 250,90 & 249,63 & 247,03 & 249,13 & 247,71 & 247,03 & BTAR* & 3,63 & 4,23 & 1,77 & 5,54 & no & yes & SYM & SYM & SYM \\
\hline & 8,05 & Jan 97 & Dez 01 & 252,08 & 249,58 & 246,03 & 250,76 & 246,87 & 246,03 & BTAR* & 4,82 & 8,61 & 1,18 & 5,10 & no & yes & SYM & SYM & SY \\
\hline & 8,05 & $\operatorname{Jan} 98$ & Dez 02 & 222,82 & 219,96 & 216,80 & 221,20 & 217,24 & 216,80 & BTAR* & 4,38 & 8,39 & 0,01 & 5,78 & no & yes & SYM & SYM & SY \\
\hline & 8,05 & Jan 99 & Dez 03 & 178,55 & 174,99 & 172,57 & 178,01 & 172,39 & 172,39 & MTAR* & 6,37 & 7,42 & & & yes,asym & yes & MTAR* & SYM & SY \\
\hline & 8,06 & Jan 97 & Dez 01 & 243,95 & 243,27 & 244,86 & 243,81 & 242,39 & 242,39 & MTAR* & 3,57 & 1,46 & & & no & yes & SYM & SYM & \\
\hline & 8,07 & Jan 97 & Dez 01 & 230,98 & 229,55 & 219,08 & 229,34 & 227,36 & 219,08 & BTAR* & 7,45 & 13,49 & 0,51 & 13,81 & yes,asym & yes & BTAR* & SYM & SYM \\
\hline & 8,07 & Jan 98 & Dez 02 & 199,78 & 198,24 & 196,97 & 197,38 & 194,41 & 194,41 & MTAR* & 5,26 & 5,15 & & & no & yes & SYM & SYM & \\
\hline & 8,07 & Jan 99 & Dez 03 & 161,48 & 160,89 & 155,74 & 161,79 & 160,63 & 155,74 & BTAR* & 5,32 & 7,15 & 0,48 & 7,19 & no & yes & SYM & SYM & SY \\
\hline & 8,08 & Jan 97 & Dez 01 & 238,34 & 237,71 & 232,45 & 237,69 & 235,01 & 232,45 & BTAR* & 4,36 & 6,90 & 0,38 & 7,51 & no & yes & SYM & SYM & BTAl \\
\hline & 8,08 & Jan 98 & Dez 02 & 206,40 & 205,08 & 193,45 & 206,11 & 203,70 & 193,45 & BTAR* & 7,15 & 14,76 & 1,28 & 15,01 & yes,asym & yes & BTAR* & SYM & BTAF \\
\hline & 8,08 & Jan 99 & Dez 03 & 167,42 & 162,96 & 158,37 & 167,06 & 164,60 & 158,37 & BTAR* & 6,31 & 11,15 & 1,00 & 10,16 & yes,asym & yes & BTAR* & SYM & BTAF \\
\hline & 8,09 & Jan 97 & Dez 01 & 244,51 & 243,08 & 237,29 & 245,01 & 241,58 & 237,29 & BTAR* & 4,95 & 7,61 & 1,19 & 8,94 & no & yes & SYM & SYM & \\
\hline & 8,09 & Jan 98 & Dez 02 & 213,18 & 211,50 & 206,21 & 213,45 & 205,43 & 205,43 & MTAR* & 6,56 & 8,45 & & & yes,asym & yes & MTAR* & SYM & SY \\
\hline & 8,09 & Jan 99 & Dez 03 & 172,86 & 169,35 & 161,20 & 171,44 & 167,28 & 161,20 & BTAR* & 6,71 & 14,03 & 0,88 & 10,63 & yes,asym & yes & BTAR* & SYM & \\
\hline & 8,10 & Jan 97 & Dez 01 & 251,24 & 248,86 & 242,97 & 250,74 & & 242,97 & BTAR* & 5,44 & 9,92 & 2,17 & 9,42 & no & yes & SYM & BTAR* & BTAl \\
\hline & 8,10 & $\operatorname{Jan} 98$ & Dez 02 & 216,99 & 214,49 & 209,57 & 215,30 & 207,43 & 207,43 & MTAR* & 7,67 & 10,20 & & & yes,asym & yes & MTAR* & BTAR* & BTAl \\
\hline & 8,10 & Jan 99 & Dez 03 & 175,78 & 173,03 & 164,78 & 173,81 & 167,79 & 164,78 & BTAR* & 7,08 & 13,85 & 1,57 & 10,42 & yes,asym & yes & BTAR* & BTAR* & BTAR \\
\hline & 8,11 & Jan 97 & Dez 01 & & & 239,83 & 246,5 & & 239,83 & BTAR* & 5,56 & 11,32 & 0,19 & 7,80 & no & yes & SYM & SYM & \\
\hline & 8,11 & Jan 98 & Dez 02 & 231,59 & 228,98 & 221,69 & 228,06 & 226,67 & 221,69 & BTAR* & 5,41 & 8,86 & 0,86 & 10,72 & no & yes & SYM & SYM & SY \\
\hline & 8,11 & Jan 99 & Dez 03 & 188,05 & 184,66 & 180,08 & 187,74 & 186,82 & 180,08 & BTAR* & 4,92 & 7,13 & 0,59 & 9,22 & no & yes & SYM & SYM & \\
\hline & 8,12 & Jan 97 & Dez 01 & 248,26 & 245,77 & 241,40 & 246,73 & 243,72 & 241,40 & BTAR* & 5,96 & 8,37 & 1,87 & 8,18 & & yes & SYM & SYM & \\
\hline
\end{tabular}




\begin{tabular}{|c|c|c|c|c|c|c|c|c|c|c|c|c|c|c|c|c|c|c|c|}
\hline & \multirow{2}{*}{ rate } & \multicolumn{2}{|c|}{ sample period } & \multirow{2}{*}{\multicolumn{5}{|c|}{ AIC }} & \multirow{2}{*}{$\begin{array}{r}\text { minimum } \\
\text { AIC }\end{array}$} & \multirow{2}{*}{$\begin{array}{r}\text { TAR-type } \\
\text { model }\end{array}$} & \multirow{2}{*}{$\begin{array}{r}\mathrm{H}_{0}: \\
\Sigma_{\mathrm{j}} \rho^{=}=0\end{array}$} & \multirow{2}{*}{$\begin{array}{r}H_{0}: \\
\rho_{1}=\rho_{2}\end{array}$} & \multirow{2}{*}{$\begin{array}{r}\mathrm{H}_{0}: \\
\rho_{1}=\rho_{3}\end{array}$} & \multirow{2}{*}{$\begin{array}{r}\mathrm{H}_{0}: \\
\rho_{2}=\rho_{3}\end{array}$} & \multirow{2}{*}{$\begin{array}{l}\text { cointe- } \\
\text { gration? }\end{array}$} & \multirow{2}{*}{$\begin{array}{l}\text { Engle-Granger } \\
\text { cointegration? }\end{array}$} & \multirow{2}{*}{$\begin{array}{r}\text { pass-through } \\
\text { model }\end{array}$} & \multirow{2}{*}{$\begin{array}{r}\text { pass-through } \\
\text { model }\end{array}$} & \multirow{2}{*}{ rolling pass- } \\
\hline & & $\begin{array}{c}\text { Sample } \\
\text { start }\end{array}$ & $\frac{r l o d}{\text { end }}$ & & & & & & & & & & & & & & & & \\
\hline $\begin{array}{l}\text { country } \\
\text { Slovak Republic }\end{array}$ & 8,01 & Jan 95 & Dez 99 & 172,52 & 169,73 & 165,62 & 173,41 & 163,66 & 163,66 & MTAR* & $\frac{j+10}{4,76}$ & 9,42 & & & $\frac{{ }^{2}}{\text { notion? }}$ & $\begin{array}{r}\text { cointegration? } \\
\text { no }\end{array}$ & $\begin{array}{r}\text { model } \\
\text { STD }\end{array}$ & $\begin{array}{r}\text { model } \\
\text { STD }\end{array}$ & $\begin{array}{r}\text { through model } \\
\text { STD }\end{array}$ \\
\hline & 8,01 & Jan 96 & Dez 00 & 206,22 & 202,44 & 201,94 & 207,36 & 199,12 & 199,12 & MTAR* & 4,18 & 7,87 & & & no & no & STD & STD & \\
\hline & 8,01 & Jan 97 & Dez 01 & 207,78 & 203,69 & 200,21 & 208,08 & 197,26 & 197,26 & MTAR* & 6,21 & 10,61 & & & yes,asym & no & MTAR* & STD & \\
\hline & 8,01 & Jan 98 & Dez 02 & 271,04 & 268,81 & 265,34 & 270,55 & 266,51 & 265,34 & BTAR* & 3,76 & 4,44 & 1,15 & 6,26 & no & yes & SYM & STD & \\
\hline & 8,01 & Jan 99 & Dez 03 & 205,41 & 199,81 & 197,23 & 207,58 & 204,10 & 197,23 & BTAR* & 5,27 & 0,66 & 11,45 & 8,86 & no & yes & SYM & STD & \\
\hline & 8,02 & Jan 95 & Dez 99 & 166,99 & 162,60 & 150,20 & 167,34 & 161,08 & 150,20 & BTAR* & 7,41 & 19,78 & 3,52 & 19,06 & yes,asym & no & BTAR* & STD & \\
\hline & 8,02 & Jan 96 & Dez 00 & 200,12 & 196,11 & 193,42 & 199,47 & 193,75 & 193,42 & BTAR* & 3,59 & 8,50 & 2,84 & 4,18 & no & no & STD & STD & \\
\hline & 8,02 & Jan 97 & Dez 01 & 209,48 & 204,88 & 202,02 & 208,41 & 200,60 & 200,60 & MTAR* & 5,73 & 9,26 & & & no & no & STD & STD & \\
\hline & 8,02 & $\operatorname{Jan} 98$ & Dez 02 & 285,15 & 283,15 & 281,43 & 284,70 & 280,46 & 280,46 & MTAR* & 4,17 & 4,37 & & & no & yes & SYM & STD & \\
\hline & 8,02 & Jan 99 & Dez 03 & 226,59 & 220,09 & 221,88 & 229,61 & 229,61 & 220,09 & TAR* & 6,90 & 10,66 & & & yes,asym & yes & TAR* & STD & \\
\hline & 8,03 & $\operatorname{Jan} 95$ & Dez 99 & 135,40 & 133,94 & 123,58 & 135,87 & 130,01 & 123,58 & BTAR* & 4,79 & 13,62 & 1,69 & 13,30 & no & no & STD & STD & \\
\hline & 8,03 & Jan 96 & Dez 00 & 152,99 & 151,69 & 142,29 & 154,61 & 150,35 & 142,29 & BTAR* & 5,15 & 12,40 & 2,94 & 11,77 & no & no & STD & STD & \\
\hline & 8,03 & Jan 97 & Dez 01 & 183,98 & 179,96 & 172,83 & 184,18 & 182,02 & 172,83 & BTAR* & 5,10 & 12,26 & 1,10 & 12,92 & no & no & STD & STD & \\
\hline & 8,03 & Jan 98 & Dez 02 & 271,88 & 266,98 & 262,48 & 271,98 & 267,86 & 262,48 & BTAR* & 4,64 & 4,44 & 5,01 & 10,70 & no & no & STD & STD & \\
\hline & 8,03 & Jan 99 & Dez 03 & 235,33 & 232,76 & 231,95 & 236,09 & 230,15 & 230,15 & MTAR* & 4,62 & 6,37 & & & no & yes & SYM & STD & \\
\hline & 8,04 & Jan 95 & Dez 99 & 62,06 & 59,35 & 47,23 & 62,41 & 60,28 & 47,23 & BTAR* & 7,55 & 16,82 & 0,10 & 17,74 & yes,asym & no & BTAR* & STD & \\
\hline & 8,04 & Jan 96 & Dez 00 & 88,11 & 86,75 & 87,04 & 88,28 & 85,28 & 85,28 & MTAR* & 2,38 & 2,70 & & & no & no & STD & STD & \\
\hline & 8,04 & Jan 97 & Dez 01 & 126,96 & 125,82 & 100,82 & 126,94 & 121,72 & 100,82 & BTAR* & 11,59 & 32,68 & 0,05 & 32,85 & yes,asym & no & BTAR* & STD & \\
\hline & 8,04 & Jan 98 & Dez 02 & 200,56 & 195,79 & 195,44 & 199,46 & 197,85 & 195,44 & BTAR* & 2,41 & 6,63 & 0,01 & 6,59 & no & no & STD & STD & \\
\hline & 8,04 & Jan 99 & Dez 03 & 196,96 & 190,51 & 190,92 & 196,34 & 191,52 & 190,51 & TAR* & 4,31 & 6,62 & & & no & yes & SYM & STD & \\
\hline & 8,05 & Jan 95 & Dez 99 & 86,79 & 80,24 & 82,24 & 87,89 & 82,66 & 80,24 & TAR* & 7,81 & 7,19 & & & yes,asym & no & TAR* & STD & \\
\hline & 8,05 & Jan 96 & Dez 00 & 128,76 & 127,92 & 122,25 & 128,70 & 126,53 & 122,25 & BTAR* & 3,54 & 7,74 & 0,83 & 7,07 & no & no & STD & STD & \\
\hline & 8,05 & Jan 97 & Dez 01 & 148,21 & 146,94 & 146,98 & 147,87 & 142,34 & 142,34 & MTAR* & 3,93 & 6,09 & & & no & no & STD & STD & \\
\hline & 8,05 & $\operatorname{Jan} 98$ & Dez 02 & 213,59 & 212,53 & 209,80 & 213,54 & 213,15 & 209,80 & BTAR* & 2,18 & 4,35 & 0,21 & 4,30 & no & no & STD & STD & \\
\hline & 8,05 & $\operatorname{Jan} 99$ & Dez 03 & 209,38 & 203,81 & 203,90 & 210,47 & 207,99 & 203,81 & TAR* & 4,55 & 6,30 & & & no & yes & SYM & STD & \\
\hline & 8,06 & Jan 95 & Dez 99 & 168,60 & 168,51 & 168,05 & 169,66 & 168,28 & 168,05 & BTAR* & 5,47 & 3,34 & 0,34 & 2,36 & no & yes & SYM & STD & \\
\hline & 8,06 & Jan 96 & Dez 00 & 173,83 & 171,37 & 173,36 & 172,15 & 170,97 & 170,97 & MTAR* & 7,30 & 3,53 & & & yes,asym & yes & MTAR* & STD & \\
\hline & 8,06 & Jan 97 & Dez 01 & 187,67 & 184,76 & 180,75 & 184,81 & 182,11 & 180,75 & BTAR* & 3,78 & 8,34 & 0,05 & 8,45 & no & no & STD & STD & \\
\hline & 8,06 & $\operatorname{Jan} 98$ & Dez 02 & 208,17 & 206,83 & 205,77 & 203,02 & 195,18 & 195,18 & MTAR* & 7,60 & 13,34 & & & yes,asym & no & MTAR* & STD & \\
\hline & 8,06 & Jan 99 & Dez 03 & 200,28 & 197,72 & 194,28 & 200,19 & 191,73 & 191,73 & MTAR* & 5,38 & 8,32 & & & no & no & STD & STD & \\
\hline & 8,07 & Jan 95 & Dez 99 & 69,25 & 60,14 & 62,12 & 71,43 & 10,30 & 10,30 & MTAR* & 90,42 & 147,58 & & & yes,asym & no & MTAR* & BTAR* & BTA \\
\hline & 8,07 & Jan 96 & Dez 00 & $-0,89$ & $-2,60$ & $-7,29$ & $-11,18$ & $-24,82$ & $-24,82$ & MTAR* & 17,10 & 28,30 & & & yes,asym & no & MTAR* & BTAR* & \\
\hline & 8,07 & Jan 97 & Dez 01 & $-1,14$ & $-2,06$ & $-2,67$ & $-7,28$ & $-8,99$ & $-8,99$ & MTAR* & 4,70 & 8,41 & & & no & no & STD & BTAR* & BTA \\
\hline & 8,07 & Jan 98 & Dez 02 & 6,25 & 5,28 & 2,54 & 5,21 & 2,62 & 2,54 & BTAR* & 2,32 & 4,92 & 0,01 & 5,28 & no & no & STD & BTAR* & BTA \\
\hline & 8,07 & Jan 99 & Dez 03 & 177,95 & 170,99 & 90,80 & 179,24 & 164,83 & 90,80 & BTAR* & 69,21 & 0,16 & 190,30 & 192,95 & yes,asym & no & BTAR* & BTAR* & BTAR \\
\hline
\end{tabular}

Note: Asymmetric cointegration is established based on the critical values reported by Enders and Siklos (2000) for 50 observations and the $10 \%$ significance level. Regarding the symmetric cointegration decision see table B2. Regarding the fullperiod pass-through model decision see table B3. The optimal 5-year rolling pass-though model is determined as follows: (1) Choose model with absolute majority (>50\%) in the rolling periods. If (1) does not lead to a choice, then (2) choose the full-period model. The STD_LL model is applied to all rates from Slovenia because these rates were found to be I $(0)$. As such, no results for Slovenia are reported in this table. 
Table B6: 5-year rolling multipliers of the optimal pass-through model

\begin{tabular}{|c|c|c|c|c|c|c|c|c|c|c|c|c|c|c|c|c|c|c|c|c|c|c|c|}
\hline \multirow[b]{2}{*}{ country } & \multirow[b]{2}{*}{ rate } & \multicolumn{2}{|c|}{ sample peri } & \multirow{2}{*}{$\begin{array}{r}\text { pass-through } \\
\text { model } \\
\end{array}$} & \multirow{2}{*}{$\begin{array}{c}\text { thres- } \\
\text { hold } \mathrm{m}\end{array}$} & \multirow{2}{*}{\multicolumn{2}{|c|}{$\begin{array}{l}\text { impact long-run } \\
\text { ultiplier multiplier } \\
\end{array}$}} & & & & & & muilt1 & 111ers for & $+0.25 \% \mathrm{~s}$ & & & pliers for & $-0.25 \% \mathrm{~s}$ & nock \\
\hline & & start & end & & & & & $1 \mathrm{mth}$ & $3 \mathrm{mths}$ & $6 \mathrm{mths}$ & $12 \mathrm{mths}$ & $1 \mathrm{mth}$ & $3 \mathrm{mths}$ & $6 \mathrm{mths}$ & $12 \mathrm{mths}$ & $1 \mathrm{mth}$ & $3 \mathrm{mths}$ & $6 \mathrm{mths}$ & $12 \mathrm{mths}$ & $1 \mathrm{mth}$ & $3 \mathrm{mths}$ & $6 \mathrm{mths}$ & $12 \mathrm{mths}$ \\
\hline $\begin{array}{l}\text { Panel A: Short-te } \\
\text { Czech Republic }\end{array}$ & $\frac{m \text { corr }}{4}$ & $\frac{\text { e loan rat }}{\text { Jan-99 }}$ & Dec-03 & MTAR* & $-0,13$ & 0,98 & 0,95 & 0,98 & 106 & 0,89 & 0,95 & 0,98 & 1,06 & 0,84 & 0,95 & 0,98 & 1,06 & 0,94 & 0,96 & 0,98 & 1,06 & 0.94 & 0,96 \\
\hline Estonia & 4 & Jan-97 & Dec-01 & SYM & & 0,40 & 0,66 & 0,36 & 0,47 & 0,56 & 0,63 & 0,36 & $\begin{array}{l}1,00 \\
0,47\end{array}$ & 0,84 & 0,63 & $\begin{array}{l}0,98 \\
0,36\end{array}$ & $\begin{array}{l}1,00 \\
0,47\end{array}$ & 0,56 & $\begin{array}{l}0,90 \\
0,63\end{array}$ & 0,36 & $\begin{array}{l}1,00 \\
0,47\end{array}$ & 0,96 & 0,60 \\
\hline & 4 & Jan-98 & Dec-02 & SYM & & 0,23 & 0,72 & 0,30 & 0,46 & 0,59 & 0,69 & 0,30 & 0,46 & 0,59 & 0,69 & 0,30 & 0,46 & 0,59 & 0,69 & 0,30 & 0,46 & 0,59 & 0,69 \\
\hline & 4 & Jan-99 & Dec-03 & SYM & & 0,32 & 0,84 & 0,42 & 0,61 & 0,75 & 0,83 & 0,42 & 0,61 & 0,75 & 0,83 & 0,42 & 0,61 & 0,75 & 0,83 & 0,42 & 0,61 & 0,75 & 0,83 \\
\hline Hungary & 4 & Jan-95 & Dec-99 & MTAR* & 0,68 & 0,41 & 1,30 & 0,67 & 1,03 & 1,43 & 1,56 & 0,67 & 1,13 & 1,55 & 1,61 & 0,67 & 1,03 & 1,43 & 1,56 & 0,67 & 1,03 & 1,43 & 1,56 \\
\hline & 4 & Jan-96 & Dec-00 & MTAR* & 0,67 & 0,56 & 1,04 & 0,86 & 1,08 & 1,09 & 1,04 & 0,86 & 1,08 & 1,09 & 1,04 & 0,86 & 1,08 & 1,09 & 1,04 & 0,86 & 1,08 & 1,09 & 1,04 \\
\hline & 4 & Jan-97 & Dec-01 & MTAR* & $-0,08$ & 0,56 & 0,93 & 0,93 & 0,93 & 0,93 & 0,93 & 0,93 & 0,94 & 0,93 & 0,93 & 0,93 & 0,93 & 0,93 & 0,93 & 0,93 & 0,94 & 0,93 & 0,93 \\
\hline & 4 & Jan-98 & Dec-02 & MTAR* & $-0,08$ & 0,57 & 0,92 & 0,94 & 0,96 & 0,94 & 0,93 & 0,94 & 0,92 & 0,92 & 0,92 & 0,94 & 0,96 & 0,94 & 0,93 & 0,94 & 0,92 & 0,92 & 0,92 \\
\hline & 4 & Jan-99 & Dec-03 & MTAR* & 0,36 & 0,72 & 0,92 & 0,88 & 0,81 & 0,96 & 0,91 & 0,88 & 0,81 & 0,96 & 0,91 & 0,88 & 0,81 & 0,96 & 0,91 & 0,88 & 0,81 & 0,96 & 0,91 \\
\hline Latvia $^{1}$ & 4 & Jan-99 & Dec-03 & STD & & $-0,05$ & $-0,04$ & $-0,04$ & $-0,04$ & $-0,04$ & $-0,04$ & $-0,04$ & $-0,04$ & $-0,04$ & $-0,04$ & $-0,04$ & $-0,04$ & $-0,04$ & $-0,04$ & $-0,04$ & $-0,04$ & $-0,04$ & $-0,04$ \\
\hline Lithuania & 4 & Jan-99 & Dec-03 & MTAR* & $-2,77$ & 0,09 & 1,13 & 0,03 & 0,28 & 0,46 & 0,74 & 0,03 & 0,28 & 0,46 & 0,74 & 0,03 & 0,28 & 0,46 & 0,74 & 0,03 & 0,28 & 0,46 & 0,74 \\
\hline $\begin{array}{l}\text { Poland } \\
\text { Pal }\end{array}$ & 4 & Jan-96 & Dec-00 & SYM & & 0,08 & 0,85 & 0,30 & 0,67 & 0,84 & 0,85 & 0,30 & 0,67 & 0,84 & 0,85 & 0,30 & 0,67 & 0,84 & 0,85 & 0,30 & 0,67 & 0,84 & 0,85 \\
\hline & 4 & Jan-97 & Dec-01 & SYM & & 0,09 & 0,86 & 0,27 & 0,61 & 0,83 & 0,87 & 0,27 & 0,61 & 0,83 & 0,87 & 0,27 & 0,61 & 0,83 & 0,87 & 0,27 & 0,61 & 0,83 & 0,87 \\
\hline & 4 & Jan-98 & Dec-02 & SYM & & 0,18 & 0,95 & 0,44 & 0,80 & 0,95 & 0,95 & 0,44 & 0,80 & 0,95 & 0,95 & 0,44 & 0,80 & 0,95 & 0,95 & 0,44 & 0,80 & 0,95 & 0,95 \\
\hline & 4 & Jan-99 & Jun- 03 & SYM & & 0,21 & 0,95 & 0,47 & 0,83 & 0,97 & 0,95 & 0,47 & 0,83 & 0,97 & 0,95 & 0,47 & 0,83 & 0,97 & 0,95 & 0,47 & 0,83 & 0,97 & 0,95 \\
\hline Slovak Republic & 4 & Jan-95 & Dec-99 & STD & & 0,29 & 0,2 & 0,17 & 0,20 & 0,21 & 0,21 & 0,17 & 0,20 & 0,21 & 0,21 & 0,17 & 0,20 & 0,21 & 0,2 & 0,17 & 0,20 & 0,21 & 0,21 \\
\hline & 4 & Jan-96 & Dec-00 & STD & & 0,33 & 0,24 & 0,21 & 0,24 & 0,24 & 0,24 & 0,21 & 0,24 & 0,24 & 0,24 & 0,21 & 0,24 & 0,24 & 0,24 & 0,21 & 0,24 & 0,24 & 0,24 \\
\hline & 4 & Jan-97 & Dec-01 & STD & & 0,34 & 0,25 & 0,21 & 0,24 & 0,25 & 0,25 & 0,21 & 0,24 & 0,25 & 0,25 & 0,21 & 0,24 & 0,25 & 0,25 & 0,21 & 0,24 & 0,25 & 0,25 \\
\hline & 4 & Jan-98 & Dec-02 & STD & & 0,35 & 0,26 & 0,22 & 0,25 & 0,26 & 0,26 & 0,22 & 0,25 & 0,26 & 0,26 & 0,22 & 0,25 & 0,26 & 0,26 & 0,22 & 0,25 & 0,26 & 0,26 \\
\hline & 4 & Jan-99 & Dec-03 & STD & & 0,31 & 0,27 & 0,27 & 0,27 & 0,27 & 0,27 & 0,27 & 0,27 & 0,27 & 0,27 & 0,27 & 0,27 & 0,27 & 0,27 & 0,27 & 0,27 & 0,27 & 0,27 \\
\hline Slovenia & 4 & Jan-93 & Dec-97 & STD LL & & 1,01 & 1,03 & 1,03 & 1,03 & 1,03 & 1,03 & 1,03 & 1,03 & 1,03 & 1,03 & 1,03 & 1,03 & 1,03 & 1,03 & 1,03 & 1,03 & 1,03 & 1,03 \\
\hline & 4 & Jan-94 & Dec-98 & STD_LL & & 0,94 & 1,05 & 1,04 & 1,05 & 1,05 & 1,05 & 1,04 & 1,05 & 1,05 & 1,05 & 1,04 & 1,05 & 1,05 & 1,05 & 1,04 & 1,05 & 1,05 & 1,05 \\
\hline & 4 & Jan-95 & Dec-99 & STD_LL & & 0,68 & 1,27 & 1,00 & 1,22 & 1,27 & 1,27 & 1,00 & 1,22 & 1,27 & 1,27 & 1,00 & 1,22 & 1,27 & 1,27 & 1,00 & 1,22 & 1,27 & 1,27 \\
\hline & 4 & Jan-96 & Dec-00 & STD_LL & & 0,35 & 1,15 & 0,59 & 0,88 & 1,06 & 1,14 & 0,59 & 0,88 & 1,06 & 1,14 & 0,59 & 0,88 & 1,06 & 1,14 & 0,59 & 0,88 & 1,06 & 1,14 \\
\hline & 4 & Jan-97 & Dec-01 & STD_LL & & 0,54 & 2,16 & 0,95 & 1,48 & 1,88 & 2,11 & 0,95 & 1,48 & 1,88 & 2,1 & 0,9 & & 1,88 & 2, & 0,95 & 1,4 & 1,88 & 2,11 \\
\hline & 4 & Jan-98 & Dec-02 & STD LL & & 0,27 & 1,34 & 0,49 & 0,80 & 1,06 & 1,27 & 0,49 & 0,80 & 1,06 & 1,27 & 0,49 & 0,80 & 1,06 & 1,27 & 0,49 & 0,80 & 1,06 & 1,27 \\
\hline & 4 & Jan-99 & Dec-03 & STD_LL & & 0,21 & 2,40 & 0,40 & 0,73 & 1,13 & 1,66 & 0,40 & 0,73 & 1,13 & 1,66 & 0,40 & 0,73 & 1,13 & 1,66 & 0,40 & 0,73 & 1,13 & 1,66 \\
\hline Panel B: Long-ter & $\mathrm{m}$ corp & e loan rats & & & & & & & & & & & & & & & & & & & & & \\
\hline Estonia & 5 & Jan-97 & Dec-01 & SYM & & 0,27 & 0,48 & 0,34 & 0,43 & 0,47 & 0,48 & 0,34 & 0,43 & 0,47 & 0,48 & 0,34 & 0,43 & 0,47 & 0,48 & 0,34 & 0,43 & 0,47 & $\overline{0,48}$ \\
\hline & 5 & Jan-98 & Dec-02 & SYM & & & & 0,45 & & 0,5 & 0,5 & 0.4 & & & & & & & & & & & 0,54 \\
\hline & 5 & Jan-99 & Dec-03 & SYM & & 0,42 & 0,63 & 0,50 & 0,59 & 0,62 & 0,63 & 0,50 & 0,59 & 0,62 & 0,63 & 0,50 & 0,59 & 0,62 & 0,6 & 0,50 & 0,59 & 0,62 & 0,63 \\
\hline Hungary & 5 & Jan-95 & Dec-99 & MTAR* & 0,14 & 0,38 & 1,24 & 0,68 & 1,04 & 1,34 & 1,32 & 0,68 & 0,92 & 1,45 & 1,36 & 0,68 & 0,80 & 1,23 & 1,21 & 0,68 & 0,92 & 1,45 & 1,36 \\
\hline & 5 & Jan-96 & Dec-00 & MTAR* & 0,05 & 0,53 & 1,07 & 0,86 & 0,89 & 1,07 & 1,07 & 0,86 & 0,86 & 1,17 & 1,1 & 0,86 & & 1,07 & 1, & 0,86 & 0,86 & 1,17 & 1,11 \\
\hline & 5 & Jan-97 & Dec-01 & MTAR* & $-0,28$ & 0,77 & 1,0 & 0,91 & 1, & 1,01 & 1,0 & 0,91 & & 1,0 & 1, & & & & & 0 & 1 & 1,01 & 1,00 \\
\hline & 5 & Jan- & Dec-02 & MTAR* & 0,01 & & & & & & & & & & & & & & & & & & 0,97 \\
\hline & 5 & Jan-99 & Dec-03 & MTAR* & $\begin{array}{r}-0,41 \\
-0,49\end{array}$ & 0,44 & 0, & 0,47 & 1,07 & 0,91 & 0,95 & 0,47 & 1,09 & 0,90 & 0,9 & 0,47 & 1,09 & 0,90 & 0,9 & 0,47 & 1,09 & 0,90 & 0,95 \\
\hline Latvia & 5 & Jan-99 & Dec-03 & MTAR* & 2,22 & 0,13 & 1, & 0,29 & 0,3 & 0 & 0,7 & 0,29 & 0,3 & & & & & 57 & & 0,29 & 0,39 & 0,57 & 0,79 \\
\hline Lithuania & 5,01 & Jan-99 & Dec-03 & SYM & & & 1, & 0,24 & 0 & 0 & 0,8 & 0,24 & 0,4 & 0,60 & & 0,2 & 0,41 & 0,60 & & 0,24 & 0,41 & 0,60 & 0,81 \\
\hline & 5,02 & Jan-99 & Dec- - & & & & & 0,28 & & & 0 & 0,28 & & & & & & 0 & & 0,28 & 0,41 & 55 & 0,70 \\
\hline Poland & 5,01 & Jan-97 & Dec-01 & SY & & & & 0 & & & & 0,2 & & & & & & & & & & & 0,86 \\
\hline THanta & 5,01 & Jan-98 & Dec-02 & SYM & & 0,13 & 0, & 0,34 & 0,69 & 0,90 & 0,9 & 0,34 & 0,69 & 0, & & 0,3 & 0 & 0,90 & & 0,34 & 0,69 & 0,90 & 0,91 \\
\hline & 5,01 & Jan- & Jun- & SY & & 0 , & 0, & 0 & 0 & 0 & 0 & 0 & 0, & & & & & 93 & & 0,43 & 0 & 0,93 & 0,90 \\
\hline & 5,02 & Jan-98 & Dec- & SYM & & & & 0,5 & & 0 & 0, & 0,5 & & & & 0,50 & 0,75 & & & 0,50 & 0,75 & 0,81 & 0,81 \\
\hline & 5,02 & Jan-99 & Jun-03 & SYM & & 0, & & 0,5 & 0 , & 0 & 0, & 0,50 & & & & 0,50 & 0,80 & 0 & & 0,50 & 0,80 & 0,89 & 0,90 \\
\hline Slovenia & 5 & Jan-93 & Dec-97 & STD LL & & & 1, & 1,0 & 1, & 1, & 1, & 1,03 & 1, & & & 1, & & & & 1,0 & 1,03 & 1,03 & 1,03 \\
\hline Sioventa & 5 & Jan-94 & Dec-98 & STD LL & & 0, & 1 , & 1,0 & 1, & 1, & 1, & 1, & & & & 1, & & & & 1, & 1, & $\begin{array}{l}1,02 \\
1,0\end{array}$ & $\begin{array}{l}1,02 \\
1,02\end{array}$ \\
\hline & 5 & Jan-95 & Dec-99 & STD LL & & & 1,26 & 1,00 & 1, & 1,2 & 1,2 & 1,0 & 1,2 & 1,2 & & 1,0 & 1,21 & 1,25 & & 1,00 & 1,21 & 1,25 & 1,26 \\
\hline & 5 & Jan-96 & Dec-00 & STD_LL & & 0,33 & $\begin{array}{l}1,09 \\
1,09\end{array}$ & 0,56 & 0,83 & 1,00 & 1,0 & 0,56 & 0,83 & 1,00 & 1, & 0,56 & 0,83 & 1,00 & & 0,56 & 0,83 & 1,00 & $\begin{array}{l}1,08 \\
1,08\end{array}$ \\
\hline & 5 & Jan-97 & Dec-01 & STD_LL & & & & 0,8 & 1, & 1, & 1 , & & & & & & & & & & & & 1,98 \\
\hline & 5 & Jan- & Dec- & STD_L & & & & 0, & 0 , & 0 , & 1 , & & 0, & 0, & 1 , & & & 0, & & & & & $\begin{array}{l}1,06 \\
1,06\end{array}$ \\
\hline & 5 & Jan-99 & Dec-03 & STD LL & & 0,23 & 2,89 & 0,44 & 0,81 & $\begin{array}{l}1,27 \\
1,27\end{array}$ & 1,91 & 0,44 & 0,81 & 1,27 & 1,91 & 0,44 & 0,81 & 1,27 & 1,91 & 0,44 & 0,81 & 1,27 & 1,91 \\
\hline Panel C: Consum & or loan & & & & & & & & & & & & & & & & & & & & & & \\
\hline Czech Republic $^{1}$ & 3 & Jan-93 & Dec-97 & SYM & & $-0,0$ & & $-0,02$ & & $-0,0$ & $-0,8$ & $-0,02$ & $-0,01$ & $-0,0$ & -0, & $-0,02$ & $-0,0$ & $-0,0$ & -0, & $-0,02$ & $-0,0$ & $-0,01$ & $-0,01$ \\
\hline & 3 & Jan-94 & Dec- -9 & SYM & & 0 & $-0,06$ & $-0,0$ & -0, & $-0,0$ & $-0,0$ & $-0,0$ & $-0,0$ & $-0,05$ & $-0,0$ & $-0,02$ & $-0,0$ & $-0,05$ & -0, & $-0,02$ & $-0,04$ & $-0,05$ & $-0,06$ \\
\hline & 3 & Jan-95 & Dec-99 & SYM & & 0,02 & $\begin{array}{r}-0,00 \\
0,10\end{array}$ & $\begin{array}{r}-r, 02 \\
0,02\end{array}$ & 0,0 & 0,04 & $\begin{array}{r}-0,0 \\
0,0\end{array}$ & $\begin{array}{r}-0,02 \\
0,02\end{array}$ & $\begin{array}{r}-0,04 \\
0,03\end{array}$ & $\begin{array}{r}-0,03 \\
0,04\end{array}$ & $\begin{array}{rl}-0,0 & 0 \\
0,0\end{array}$ & $\begin{array}{r}-0,02 \\
0,02\end{array}$ & $\begin{array}{r}-0,04 \\
0,03\end{array}$ & $\begin{array}{r}-0,03 \\
0,04\end{array}$ & $\begin{array}{r}-0,0 \\
0,0\end{array}$ & $\begin{array}{r}-r, 02 \\
0,02\end{array}$ & $\begin{array}{r}-r, 04 \\
0,03\end{array}$ & $\begin{aligned}-0,03 \\
0,04\end{aligned}$ & $\begin{array}{r}-0,00 \\
0,06\end{array}$ \\
\hline & 3 & Jan-96 & Dec-00 & SYM & & & & 0,0 & 0, & 0, & 0, & & & 0, & & 0,0 & 0, & & & & & & \\
\hline & 3 & Jan- & Dec- & & & & & & & & & & & & & & & & & & & & 0, \\
\hline & 3 & Jan-98 & Dec-02 & SYM & & 0,17 & 0,2 & 0,17 & 0,21 & 0,22 & 0,22 & 0,17 & 0,21 & 0,22 & 0,2 & 0,17 & 0,21 & 0,22 & 0,22 & 0,17 & 0,21 & 0,22 & 0,22 \\
\hline & 3 & Jan-99 & Dec-03 & SYM & & 0,27 & 0,23 & 0,25 & 0,24 & 0,23 & 0,23 & 0,25 & 0,24 & 0,23 & 0,23 & 0,25 & 0,24 & 0,23 & 0,23 & 0,25 & 0,24 & 0,23 & 0,23 \\
\hline
\end{tabular}


Table B6: 5-year rolling multipliers of the optimal pass-through model

\begin{tabular}{|c|c|c|c|c|c|c|c|c|c|c|c|c|c|c|c|c|c|c|c|c|c|c|c|}
\hline \multirow{3}{*}{ country } & \multirow[b]{2}{*}{ rate } & \multicolumn{2}{|c|}{ sample period } & \multirow{2}{*}{$\begin{array}{r}\text { pass-through } \\
\text { model }\end{array}$} & \multirow{2}{*}{$\begin{array}{l}\text { thres- } \\
\text { hold }\end{array}$} & \multirow{2}{*}{\multicolumn{2}{|c|}{$\begin{array}{l}\text { impact long-run } \\
\text { ultiplier multiplier }\end{array}$}} & \multicolumn{4}{|c|}{ multipliers for $+1 \%$ shock } & $\mathrm{mul}$ & ipliers fo & $-1 \% \mathrm{sh}$ & & multip & liers for & $-0.25 \% \mathrm{~s}$ & hock & multip & liers for & $-0.25 \% \mathrm{~s}$ & 1ock \\
\hline & & start & end & & & & & $1 \mathrm{mth}$ & $3 \mathrm{mths}$ & $6 \mathrm{mths}$ & $12 \mathrm{mths}$ & $1 \mathrm{mth}$ & $3 \mathrm{mths}$ & $6 \mathrm{mths}$ & $12 \mathrm{mths}$ & $1 \mathrm{mth}$ & $3 \mathrm{mths}$ & $6 \mathrm{mths}$ & $12 \mathrm{mths}$ & $1 \mathrm{mth}$ & 3 mths & $6 \mathrm{mths}$ & $12 \mathrm{mths}$ \\
\hline & 3 & Jan-97 & Dec-01 & & 0,77 & $-0,21$ & 0,14 & $-0,09$ & 0,14 & 0,12 & 0,14 & $-0,09$ & 0,14 & 0,12 & 0,14 & $-0,09$ & 0,14 & 0,12 & 0,14 & $-0,09$ & 0,14 & 0,12 & 0,14 \\
\hline & 3 & Jan-98 & Dec-02 & MTAR* & $-0,80$ & $-0,24$ & 0,24 & $-0,15$ & 0,08 & 0,20 & 0,24 & $-0,15$ & 0,08 & 0,20 & 0,24 & $-0,15$ & 0,08 & 0,20 & 0,24 & $-0,15$ & 0,08 & 0,20 & 0,24 \\
\hline & 3 & Jan-99 & Dec-03 & MTAR* & $-1,99$ & $-0,57$ & 0,27 & $-0,25$ & 0,08 & 0,54 & 0,15 & $-0,25$ & 0,08 & 0,54 & 0,15 & $-0,25$ & 0,08 & 0,54 & 0,15 & $-0,25$ & 0,08 & 0,54 & 0,15 \\
\hline Hungary & 3 & Jan-95 & Dec-99 & SYM & & 0,23 & 0,67 & 0,42 & 0,61 & 0,66 & 0,67 & 0,42 & 0,61 & 0,66 & 0,67 & 0,42 & 0,61 & 0,66 & 0,67 & 0,42 & 0,61 & 0,66 & 0,67 \\
\hline & 3 & Jan-96 & Dec-00 & SYM & & 0,23 & 0,60 & 0,38 & 0,52 & 0,59 & 0,60 & 0,38 & 0,52 & 0,59 & 0,60 & 0,38 & 0,52 & 0,59 & 0,60 & 0,38 & 0,52 & 0,59 & 0,60 \\
\hline & 3 & Jan-97 & Dec-01 & SYM & & 0,14 & 0,43 & 0,18 & 0,25 & 0,32 & 0,39 & 0,18 & 0,25 & 0,32 & 0,39 & 0,18 & 0,25 & 0,32 & 0,39 & 0,18 & 0,25 & 0,32 & 0,39 \\
\hline & 3 & Jan-98 & Dec-02 & SYM & & $-0,03$ & 0,31 & 0,04 & 0,13 & 0,21 & 0,28 & 0,04 & 0,13 & 0,21 & 0,28 & 0,04 & 0,13 & 0,21 & 0,28 & 0,04 & 0,13 & 0,21 & 0,28 \\
\hline & 3 & Jan-99 & Dec-03 & SYM & & 0,07 & 0,27 & 0,12 & 0,19 & 0,24 & 0,27 & 0,12 & 0,19 & 0,24 & 0,27 & 0,12 & 0,19 & 0,24 & 0,27 & 0,12 & 0,19 & 0,24 & 0,27 \\
\hline Latvia & 3,01 & Jan-99 & Dec-03 & SYM & & 0,33 & 0,82 & 0,37 & 0,54 & 0,68 & 0,78 & 0,37 & 0,54 & 0,68 & 0,78 & 0,37 & 0,54 & 0,68 & 0,78 & 0,37 & 0,54 & 0,68 & 0,78 \\
\hline & 3,02 & Jan-99 & Dec-03 & SYM & & 0,66 & 2,76 & 0,78 & 1,15 & 1,57 & 2,10 & 0,78 & 1,15 & 1,57 & 2,10 & 0,78 & 1,15 & 1,57 & 2,10 & 0,78 & 1,15 & 1,57 & 2,10 \\
\hline Lithuania & 3,01 & Jan-99 & Dec-03 & MTAR* & $-0,26$ & 0,24 & 0,82 & $-0,23$ & 0,03 & 0,20 & 0,49 & $-0,23$ & 0,17 & 0,22 & 0,50 & $-0,23$ & 0,17 & 0,24 & 0,51 & $-0,23$ & 0,17 & 0,24 & 0,51 \\
\hline Lithuania $^{1}$ & 3,02 & Jan-99 & Dec-03 & SYM & & 0,00 & 0,77 & 0,06 & 0,15 & 0,27 & 0,44 & 0,06 & 0,15 & 0,27 & 0,44 & 0,06 & 0,15 & 0,27 & 0,44 & 0,06 & 0,15 & 0,27 & 0,44 \\
\hline Poland & 3 & Jan-97 & Dec-01 & MTAR* & 0,63 & 0,09 & 0,61 & 0,24 & 0,39 & 0,58 & 0,66 & 0,24 & 0,39 & 0,58 & 0,66 & 0,24 & 0,39 & 0,58 & 0,66 & 0,24 & 0,39 & 0,58 & 0,66 \\
\hline & 3 & Jan-98 & Dec-02 & MTAR* & 1.84 & 0.12 & 0.62 & 0.32 & 0.47 & 0.61 & 0.64 & 0.32 & 0.47 & 0,61 & 0,64 & 0.32 & 0.47 & 0.61 & 0.64 & 0.32 & 0.47 & 0,61 & 0.64 \\
\hline & 3 & Jan-99 & Jun-03 & MTAR* & 0,86 & 0,10 & 0,46 & 0,23 & 0,37 & 0,55 & 0,46 & 0,23 & 0,37 & 0,55 & 0,46 & 0,23 & 0,37 & & & & & 0,55 & 0,46 \\
\hline Slovak Republic & 3 & Jan-95 & Dec-99 & SYM & & $-0,08$ & $-0,18$ & $-0,09$ & $-0,12$ & $-0,14$ & $-0,17$ & $-0,09$ & $-0,12$ & $-0,14$ & $-0,17$ & $-0,09$ & $-0,12$ & $-0,14$ & $-0,17$ & $-0,09$ & $-0,12$ & $-0,14$ & $-0,17$ \\
\hline & 3 & Jan-96 & Dec-00 & SYM & & $-0,04$ & 0,05 & $-0,01$ & 0,00 & 0,02 & 0,04 & $-0,01$ & 0,00 & 0,02 & 0,04 & $-0,01$ & 0,00 & 0,02 & 0,04 & $-0,01$ & 0,00 & 0,02 & 0,04 \\
\hline & 3 & Jan-97 & Dec-01 & SYM & & $-0,05$ & 0,12 & 0,00 & 0,02 & 0,05 & 0,09 & 0,00 & 0,02 & 0,05 & 0,09 & 0,00 & 0,02 & 0,05 & 0,09 & 0,00 & 0,02 & 0,05 & 0,09 \\
\hline & 3 & Jan-98 & Dec-02 & SYM & & $-0,05$ & 0,05 & $-0,01$ & 0,01 & 0,03 & 0,04 & $-0,01$ & 0,01 & 0,03 & 0,04 & $-0,01$ & & & & & & 0,03 & 0,04 \\
\hline & 3 & Jan-99 & Dec-03 & SYM & & $-0,05$ & $-0,08$ & $-0,05$ & $-0,06$ & $-0,07$ & $-0,08$ & $-0,05$ & $-0,06$ & $-0,07$ & $-0,08$ & $-0,05$ & $-0,06$ & $-0,07$ & -0, & $-0,05$ & $-0,06$ & $-0,07$ & $-0,08$ \\
\hline Slovenia & 3 & Jan-93 & Dec-97 & STD_LL & & 1,01 & 1,03 & 1,03 & 1,03 & 1,03 & 1,03 & 1,03 & 1,03 & 1,03 & 1,03 & 1,03 & 1,03 & 1,03 & 1,03 & 1,03 & 1,03 & 1,03 & 1,03 \\
\hline & 3 & Jan-94 & Dec-98 & STD_LL & & 0,93 & 1,09 & 1,07 & 1,09 & 1,09 & 1,09 & 1,07 & 1,09 & 1,09 & 1,09 & 1,07 & 1,09 & 1,09 & 1,09 & 1,07 & 1,09 & 1,09 & 1,09 \\
\hline & 3 & Jan-95 & Dec-99 & STD LL & & 0,70 & 1,33 & 1,04 & 1,27 & 1,33 & 1,33 & 1,04 & 1,27 & 1,33 & 1,33 & 1,04 & 1,27 & 1,33 & 1,33 & 1,04 & 1,27 & 1,33 & 1,33 \\
\hline & 3 & Jan-96 & Dec-00 & STD LL & & 0,39 & 1,2 & 0,66 & 0,97 & 1,15 & 1,23 & 0,66 & & & 1,2 & 0,66 & & & & & & & 1,23 \\
\hline & 3 & Jan-97 & Dec-01 & STD_LL & & 0,59 & 2,18 & 1,03 & 1,57 & 1,94 & 2,15 & 1,03 & 1,57 & 1,94 & 2,15 & 1,03 & 1,57 & 1,94 & 2,15 & 1,03 & 1,57 & 1,94 & 2,15 \\
\hline & 3 & Jan-98 & Dec-02 & STD_LL & & 0,25 & 1,16 & 0,44 & 0,72 & 0,94 & 1,11 & 0,44 & 0,72 & 0,94 & 1,11 & 0,44 & 0,72 & 0,94 & 1,11 & 0,44 & 0,72 & 0,94 & 1,11 \\
\hline & 3 & Jan-99 & Dec-03 & STD_LL & & 0,16 & 1,35 & 0,30 & 0,53 & 0,78 & 1,08 & 0,30 & 0,53 & 0,78 & 1,08 & 0,30 & 0,53 & 0,78 & 1,08 & 0,30 & 0,53 & 0,78 & 1,08 \\
\hline anel D: & e rates & & & & & & & & & & & & & & & & & & & & & & \\
\hline Estonia & 2 & Jan-97 & \begin{tabular}{|l|} 
Dec-01 \\
\end{tabular} & SYM & & 0,03 & & 0,05 & , & 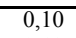 & 0,1 & 0,05 & & & & 0,05 & & & & & & 0,10 & 0,12 \\
\hline & 2 & Jan-98 & Dec-02 & SYM & & 0,04 & 0,23 & 0,06 & 0,09 & 0,12 & 0,17 & 0,06 & 0,09 & 0,12 & 0,1 & 0,06 & 0,09 & 0,12 & 0,1 & 0,06 & 0,09 & 0,12 & 0,17 \\
\hline & 2 & Jan-99 & Dec-03 & SYM & & $-0,01$ & 0,73 & 0,02 & 0,05 & 0,09 & 0,17 & 0,02 & 0,05 & 0,09 & 0,17 & 0,02 & 0,05 & 0,09 & 0,1 & 0,02 & 0,05 & 0,09 & 0,17 \\
\hline Hungary & 2 & Jan-95 & Dec-99 & MTAR* & 0,57 & 0,08 & 0 , & 0,47 & 13 & 0,30 & 0,60 & 0,47 & & & 0,60 & & & & & & & 0,30 & 0,60 \\
\hline & 2 & Jan-96 & Dec- & MTAR* & 0,88 & 0,01 & & 0,53 & & 0,74 & 0,85 & 0,53 & & & 0,8 & & & & & & & 74 & 0,85 \\
\hline & 2 & & & MTAR* & 1,25 & $-0,1$ & & & & & & 0,60 & & & & & & & & & & 06 & 1,09 \\
\hline & 2 & Jan-98 & Dec-02 & MTAR* & 1,00 & $\begin{array}{l}-0,11 \\
-10\end{array}$ & 1, & 0,31 & 0,84 & 1,00 & 1,16 & 0,31 & 1,13 & 1,19 & 1,24 & 0,31 & 0,84 & 1,00 & $\begin{array}{l}1,1 \\
1,1\end{array}$ & 0,31 & 0,84 & 1,00 & 1,16 \\
\hline & 2 & Jan-99 & Dec-03 & $\mathrm{AR}^{*}$ & 1,42 & 0,2 & & 0,26 & & & & 0,26 & & & 1,0 & 0,26 & & & & & 47 & 73 & 1,03 \\
\hline Latvia & 2 & Jan-99 & Dec & MTAR* & 0,68 & $-0,04$ & 1,01 & $-0,03$ & & 0,27 & 0,50 & $-0,03$ & 0 , & & & -0, & & & & -0 , & 11 & 0,27 & 0,50 \\
\hline Lithuania & 2,01 & & & SYM & & & & 0,09 & & & & 0,09 & & & & & & & & & & & 0,41 \\
\hline & 2,02 & Jan-99 & Dec-03 & STD & & 0,18 & & & & & & & & & & & & & & & & 13 & 0,13 \\
\hline Slovenia & 2 & Jan-93 & Dec-97 & STD_LL & & 0,87 & & 0,81 & & & & & & & & & & & & & 31 & 0,81 & 0,81 \\
\hline & 2 & & Dec & STD_L & & & & & & & & & & & & & & & & & 0,89 & 0,89 & 0,89 \\
\hline & 2 & & Dec- & STD ${ }^{-}$LL & & 0,68 & & 0,96 & & & 1,1 & 0,96 & & & & & & & & & & 1,16 & 1,16 \\
\hline & 2 & Jan- & Dec & STD I & & & & 0, & & & & 0, & & & & & & & & & & & 1,09 \\
\hline & 2 & Jan-97 & Dec-01 & STD I & & 0,5 & & 1,01 & & 1,8 & 2, & 1,0 & & & & & & & & & & 37 & 2,03 \\
\hline & 2 & & Dec- & STD_I & & 0 , & & 0,48 & & & & 0,48 & & & 1 , & 0,48 & & & & & 0,76 & 0,98 & 1,12 \\
\hline & 2 & Jan-99 & Dec-03 & STD LL & & 0,19 & 1,82 & 0,36 & 0,66 & 0,99 & 1,39 & 0,36 & 0,66 & 0,99 & 1,39 & 0,36 & 0,66 & 0,99 & 1,39 & 0,36 & 0,66 & 0,99 & 1,39 \\
\hline anel E: Current & 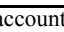 & osit rates & & & & & & & & & & & & & & & & & & & & & \\
\hline Hungary & 7 & Jan-95 & & & 0,1 & & & -0, & & & & & & & & -0 & & & & & & & 0,24 \\
\hline & 7 & & Dec- & & 0,1 & & & 0,2 & & & & & & & & & & & & & & & 0,38 \\
\hline & 7 & & & & & & & & & & & & & & & & & & & & & 0,47 & 0,47 \\
\hline & 7 & Jan-98 & Dec-02 & MTAR* & 0,09 & 0,16 & 0,49 & 0,34 & 0,40 & 0,44 & 0,48 & 0,34 & 0,39 & 0,44 & 0,47 & 0,34 & 0,41 & 0,45 & 0,48 & 0,34 & 0,41 & 0,45 & 0,48 \\
\hline & 7 & Jan-99 & Dec-03 & MTAR* & $-0,25$ & 0,13 & 0,52 & 0,20 & 0,31 & 0,41 & 0,49 & 0,20 & 0,32 & 0,42 & 0,49 & 0,20 & 0,32 & 0,42 & 0,49 & 0,20 & 0,32 & 0,42 & 0,49 \\
\hline Poland ${ }^{1}$ & 7,01 & Jan-97 & Dec-01 & SYM & & 0,08 & 0,63 & 0,13 & 0,20 & 0,28 & 0,40 & 0,13 & 0,20 & 0,28 & 0,40 & 0,13 & 0,20 & 0,28 & 0,40 & 0,13 & 0,20 & 0,28 & 0,40 \\
\hline & 7,01 & Jan-98 & Dec-02 & SYI & & 0,12 & 0,67 & 0,19 & 0,27 & 0,36 & 0,4 & 0,19 & 0,27 & 0,36 & 0 & & 0,27 & 0,36 & & & 0,27 & 0,36 & 0,49 \\
\hline & 7,01 & Jan-99 & Jun-03 & SYM & & 0,07 & 0,55 & 0,07 & 0,04 & $-0,01$ & $-0,13$ & 0,07 & 0,04 & $-0,01$ & $-0,13$ & 0,07 & 0,04 & $-0,01$ & $-0,13$ & 0,07 & 0,04 & $-0,01$ & $-0,13$ \\
\hline Poland $^{1}$ & 7,02 & Jan-97 & Dec-01 & SYM & & 0,03 & 0,04 & 0,04 & 0,04 & 0,04 & 0,04 & 0,04 & 0,04 & 0,04 & 0,04 & 0,04 & 0,04 & 0,04 & 0,04 & 0,04 & 0,04 & 0,04 & 0,04 \\
\hline & 7,02 & Jan-98 & & SYI & & & 0,1 & & & & & 0,11 & & & & & & & & & & 0,13 & 0,13 \\
\hline & 7,02 & Jan-99 & Jun-03 & SYM & & 0,10 & 0,16 & 0,13 & 0,15 & 0,16 & 0,16 & 0,13 & 0,15 & 0,16 & 0,16 & 0,13 & 0,15 & 0,16 & 0,16 & 0,13 & 0,15 & 0,16 & 0,16 \\
\hline
\end{tabular}


Table B6: 5-year rolling multipliers of the optimal pass-through model

\begin{tabular}{|c|c|c|c|c|c|c|c|c|c|c|c|c|c|c|c|c|c|c|c|c|c|c|c|}
\hline \multirow{3}{*}{\multicolumn{2}{|c|}{ country rate }} & & \multirow{2}{*}{$\begin{array}{r}\text { pass-through } \\
\text { model }\end{array}$} & \multirow{2}{*}{\multicolumn{3}{|c|}{$\begin{array}{c}\text { thres- } \text { impact long-run } \\
\text { hold multiplier multiplier }\end{array}$}} & & & & & & & & & & \\
\hline & & start & end & & & & & $1 \mathrm{mth}$ & $3 \mathrm{mths}$ & $6 \mathrm{mths}$ & $12 \mathrm{mths}$ & $1 \mathrm{mth}$ & $3 \mathrm{mths}$ & $6 \mathrm{mths}$ & $12 \mathrm{mths}$ & $1 \mathrm{mth}$ & $3 \mathrm{mths}$ & $6 \mathrm{mths}$ & $12 \mathrm{mths}$ & $1 \mathrm{mth}$ & $3 \mathrm{mths}$ & $6 \mathrm{mths}$ & $12 \mathrm{mths}$ \\
\hline & & & & & & & & & & & & & & & & & & & & & & & \\
\hline Czech Republic & 9 & Jan-93 & Dec-97 & STD & & 0,00 & 0,00 & 0,00 & 0,00 & 0,00 & 0,00 & 0,00 & 0,00 & 0,00 & 0,00 & 0,00 & 0,00 & 0,00 & 0,00 & 0,00 & 0,00 & 0,00 & 0,00 \\
\hline & 9 & Jan-94 & Dec-98 & STD & & 0,00 & 0,00 & 0,00 & 0,00 & 0,00 & 0,00 & 0,00 & 0,00 & 0,00 & 0,00 & 0,00 & 0,00 & 0,00 & 0,00 & 0,00 & 0,00 & 0,00 & 0,00 \\
\hline & 9 & Jan-95 & Dec-99 & STD & & 0,00 & 0,00 & 0,00 & 0,00 & 0,00 & 0,00 & 0,00 & 0,00 & 0,00 & 0,00 & 0,00 & 0,00 & 0,00 & 0,00 & 0,00 & 0,00 & 0,00 & 0,00 \\
\hline & 9 & Jan-96 & Dec-00 & STD & & 0,00 & 0,00 & 0,00 & 0,00 & 0,00 & 0,00 & 0,00 & 0,00 & 0,00 & 0,00 & 0,00 & 0,00 & 0,00 & 0,00 & 0,00 & 0,00 & 0,00 & 0,00 \\
\hline & 9 & Jan-97 & Dec-01 & STD & & 0,00 & 0,00 & 0,00 & 0,00 & 0,00 & 0,00 & 0,00 & 0,00 & 0,00 & 0,00 & 0,00 & 0,00 & 0,00 & 0,00 & 0,00 & 0,00 & 0,00 & 0,00 \\
\hline & 9 & Jan-98 & Dec-02 & STD & & 0,03 & 0,02 & 0,02 & 0,02 & 0,02 & 0,02 & 0,02 & 0,02 & 0,02 & 0,02 & 0,02 & 0,02 & 0,02 & 0,02 & 0,02 & 0,02 & 0,02 & 0,02 \\
\hline & 9 & Jan-99 & Dec-03 & STD & & 0,08 & 0,06 & 0,06 & 0,06 & 0,06 & 0,06 & 0,06 & 0,06 & 0,06 & 0,06 & 0,06 & 0,06 & 0,06 & 0,06 & 0,06 & 0,06 & 0,06 & 0,06 \\
\hline Lithuania $^{1}$ & 9 & Jan-99 & Dec-03 & SYM & & $-0,09$ & 0,56 & 0,03 & 0,07 & 0,14 & 0,27 & 0,03 & 0,07 & 0,14 & 0,27 & 0,03 & 0,07 & 0,14 & 0,27 & 0,03 & 0,07 & 0,14 & 0,27 \\
\hline Slovak Republic & 9 & Jan-95 & Dec-99 & STD & & 0,01 & 0,00 & 0,00 & 0,00 & 0,00 & 0,00 & 0,00 & 0,00 & 0,00 & 0,00 & 0,00 & 0,00 & 0,00 & 0,00 & 0,00 & 0,00 & 0,00 & 0,00 \\
\hline & 9 & Jan-96 & Dec-00 & STD & & 0,00 & 0,00 & 0,00 & 0,00 & 0,00 & 0,00 & 0,00 & 0,00 & 0,00 & 0,00 & 0,00 & 0,00 & 0,00 & 0,00 & 0,00 & 0,00 & 0,00 & 0,00 \\
\hline & 9 & Jan-97 & Dec-01 & STD & & 0,00 & 0,00 & 0,00 & 0,00 & 0,00 & 0,00 & 0,00 & 0,00 & 0,00 & 0,00 & 0,00 & 0,00 & 0,00 & 0,00 & 0,00 & 0,00 & 0,00 & 0,00 \\
\hline & 9 & Jan-98 & Dec-02 & STD & & 0,01 & 0,00 & 0,00 & 0,00 & 0,00 & 0,00 & 0,00 & 0,00 & 0,00 & 0,00 & 0,00 & 0,00 & 0,00 & 0,00 & 0,00 & 0,00 & 0,00 & 0,00 \\
\hline & 9 & Jan-99 & Dec-03 & STD & & 0,01 & 0,01 & 0,01 & 0,01 & 0,01 & 0,01 & 0,01 & 0,01 & 0,01 & 0,01 & 0,01 & 0,01 & 0,01 & 0,01 & 0,01 & 0,01 & 0,01 & 0,01 \\
\hline Slovenia & 9 & Jan-93 & Dec-97 & STD_LL & & 0,72 & 0,77 & 0,77 & 0,77 & 0,77 & 0,77 & 0,77 & 0,77 & 0,77 & 0,77 & 0,77 & 0,77 & 0,77 & 0,77 & 0,77 & 0,77 & 0,77 & 0,77 \\
\hline & 9 & Jan-94 & Dec-98 & STD LL & & 0,53 & 0,84 & 0,72 & 0,82 & 0,84 & 0,84 & 0,72 & 0,82 & 0,84 & 0,84 & 0,72 & 0,82 & 0,84 & 0,84 & 0,72 & 0,82 & 0,84 & 0,84 \\
\hline & 9 & Jan-95 & Dec-99 & STD_LL & & 0,11 & 0,31 & 0,18 & 0,25 & $\begin{array}{l}0,07 \\
0,29\end{array}$ & 0,31 & 0,18 & 0,25 & 0,29 & 0,31 & 0,18 & 0,25 & 0,29 & 0,31 & 0,18 & 0,25 & 0,29 & 0,31 \\
\hline & 9 & Jan-96 & Dec-00 & STD LL & & 0,04 & 0,04 & 0,04 & 0,04 & 0,04 & 0,04 & 0,04 & 0,04 & 0,04 & 0,04 & 0,04 & 0,04 & 0,04 & 0,04 & 0,04 & 0,04 & 0,04 & 0,04 \\
\hline & 9 & Jan-97 & Dec-01 & STD LL & & 0,00 & 0,00 & 0,00 & 0,00 & 0,00 & 0,00 & 0,00 & 0,00 & 0,00 & 0,00 & 0,00 & 0,00 & 0,00 & 0,00 & 0,00 & 0,00 & 0,00 & 0,00 \\
\hline & 9 & Jan-98 & Dec-02 & STD_LL & & $-0,01$ & $-0,02$ & $-0,01$ & $-0,01$ & $-0,02$ & $-0,02$ & $-0,01$ & $-0,01$ & $-0,02$ & $-0,02$ & $-0,01$ & $-0,01$ & $-0,02$ & $-0,02$ & $-0,01$ & $-0,01$ & $-0,02$ & $-0,02$ \\
\hline & 9 & Jan-99 & Dec-03 & STD_LL & & $-0,01$ & $-0,03$ & $-0,01$ & $-0,02$ & $-0,02$ & $-0,03$ & $-0,01$ & $-0,02$ & $-0,02$ & $-0,03$ & $-0,01$ & $-0,02$ & $-0,02$ & $-0,03$ & $-0,01$ & $-0,02$ & $-0,02$ & $-0,03$ \\
\hline Panel G: Time de & posit ra & & & & & & & & & & & & & & & & & & & & & & \\
\hline Czech Republic & 8,01 & Jan-93 & Dec-97 & MTAR* & 0,03 & 0,33 & 0,20 & 0,16 & 0,21 & 0,28 & 0,20 & 0,16 & 0,21 & 0,28 & 0,20 & 0,16 & 0,21 & 0,28 & 0,20 & 0,16 & 0,21 & 0,28 & 0,20 \\
\hline & 8,01 & Jan-94 & Dec-98 & MTAR* & 0,61 & 0,35 & 0,25 & 0,18 & 0,26 & 0,34 & 0,28 & 0,18 & 0,26 & 0,34 & 0,28 & 0,18 & 0,26 & 0,34 & 0,28 & 0,18 & 0,26 & 0,34 & 0,28 \\
\hline & 8,01 & Jan-95 & Dec-99 & MTAR* & $-1,73$ & 0,36 & 0,42 & 0,19 & 0,32 & 0,48 & 0,4 & 0,19 & 0,32 & 0,48 & 0,47 & 0,19 & 0,32 & 0,48 & & 0,19 & 0,32 & 0,48 & 0,47 \\
\hline & 8,01 & Jan-96 & Dec-00 & MTAR* & $-1,02$ & 0,36 & 0,51 & 0,19 & 0,33 & 0,52 & 0,55 & 0,19 & 0,33 & 0,52 & 0,55 & 0,19 & 0,33 & 0,52 & 0,55 & 0,19 & 0,33 & 0,52 & 0,55 \\
\hline & 8,01 & Jan-97 & Dec-01 & MTAR* & $-1,03$ & 0,36 & 0,55 & 0,19 & 0,33 & 0,52 & 0,56 & 0,19 & 0,33 & 0,52 & 0,56 & 0,19 & 0,33 & 0,52 & 0,56 & 0,19 & 0,33 & 0,52 & 0,56 \\
\hline & 8,01 & Jan-98 & Dec-02 & MTAR* & 0,14 & 0,41 & 0,80 & 0,62 & 0,84 & 0,83 & 0,81 & 0,62 & 0,85 & 0,84 & 0,81 & 0,62 & 0,83 & 0,82 & 0,80 & 0,62 & 0,83 & 0,82 & 0,80 \\
\hline & 8,01 & Jan-99 & Dec-03 & MTAR* & 0,09 & 0,60 & 0,94 & 0,61 & 0,84 & 0,76 & 0,81 & 0,61 & 0,84 & 0,76 & 0,81 & 0,61 & 0,84 & 0,76 & 0,81 & 0,61 & 0,84 & 0,76 & 0,81 \\
\hline & 8,02 & Jan-93 & Dec-97 & BTAR* & $-0,02$ & & & 0,06 & 0,0 & 0,08 & 0,0 & 0,06 & & & & 0,0 & & & & & & 0,08 & 0,06 \\
\hline & 8,02 & Jan-94 & Dec-98 & BTAR* & $-0,02$ & 0,06 & 0,01 & 0,06 & 0,07 & 0,05 & 0,02 & 0,06 & 0,08 & 0,06 & 0,02 & 0,06 & 0,07 & 0,05 & 0,02 & 0,06 & 0,07 & 0,05 & 0,02 \\
\hline & 8,02 & Jan-95 & Dec-99 & BTAR* & $-0,04$ & 0,07 & 0,29 & 0,09 & 0,13 & 0,20 & 0,27 & 0,09 & 0,15 & 0,21 & 0,27 & 0,09 & 0,13 & 0,20 & 0,27 & 0,09 & 0,15 & 0,21 & 0,27 \\
\hline & 8,02 & Jan-96 & Dec-00 & BTAR* & $-0,49$ & 0,08 & 0,43 & 0,10 & 0,18 & 0,28 & 0,38 & 0,10 & 0,18 & 0,28 & 0,38 & 0,10 & 0,18 & 0,28 & 0,3 & 0,10 & 0,18 & 0,28 & 0,38 \\
\hline & 8,02 & Jan-97 & Dec-01 & BTAR* & $-0,57$ & 0,09 & 0,49 & 0,10 & 0,20 & 0,31 & 0,43 & 0,10 & 0,20 & 0,31 & 0 & 0,10 & 0,20 & 0,31 & & 0,10 & 0,2 & 0,31 & 0,43 \\
\hline & 8,02 & Jan-98 & Dec-02 & BTAR* & 0,72 & & & 0,3 & 0, & 0,46 & & 0,3 & 0,9 & & & & & & & & & 0,37 & 0,49 \\
\hline & 8,02 & Jan-99 & Dec-03 & BTAR* & 0,04 & 0,47 & 0,98 & 0,48 & 0,56 & 0,65 & 0,78 & $\begin{array}{l}0,34 \\
0,48\end{array}$ & 0,54 & 0,64 & 0,77 & $\begin{array}{l}0,34 \\
0,48\end{array}$ & 0,56 & 0,65 & $\begin{array}{l}0,49 \\
0,78\end{array}$ & 0,48 & 0,54 & 0,64 & $\begin{array}{l}0,49 \\
0,77\end{array}$ \\
\hline & 8,03 & Jan-93 & Dec-97 & STD & & $-0,01$ & $-0,01$ & $-0,01$ & $-0,01$ & $-0,01$ & $-0,01$ & $-0,01$ & $-0,01$ & $-0,01$ & $-0,0$ & $-0,01$ & $-0,01$ & $-0,01$ & $-0,01$ & $-0,01$ & $-0,01$ & $-0,01$ & $-0,01$ \\
\hline & 8,03 & Jan-94 & Dec-98 & STD & & 0,00 & 0,00 & 0,00 & 0,00 & 0,00 & 0,00 & 0,00 & 0,00 & 0,00 & $0, \mathrm{c}$ & 0,00 & 0,00 & 0,00 & & 0,00 & 0,00 & 0,00 & 0,00 \\
\hline & & Jan-95 & Dec- & STD & & & & 0,0 & 0 & 0,00 & & 0,00 & 0, & & & 0,00 & & 0,00 & & & & 0,00 & 0,00 \\
\hline & 8,03 & Jan & c-00 & STI & & & & 0, & & & & 0, & & & & 0, & & & & & & 01 & 0,01 \\
\hline & $\begin{array}{l}8,03 \\
8,03\end{array}$ & Jan-97 & Dec-01 & STD & & 0,00 & 0,00 & 0,00 & 0,00 & 0,00 & 0, & 0,00 & 0,00 & 0,00 & 0,0 & 0,00 & 0,00 & 0,00 & 0,0 & 0,00 & 0,00 & 0,00 & 0,00 \\
\hline & 8,03 & Jan- & Dec-02 & STD & & 0, & 0, & 0, & 0, & 0, & 0 & 0,08 & 0,0 & 0,09 & 0, & 0,0 & 0,09 & 0,09 & & 0, & 9 & 0,09 & 0,09 \\
\hline & 8,03 & Jan-99 & Dec- & STD & & 0,06 & 0, & 0,08 & 0,0 & 0,09 & 0, & 0,08 & 0,09 & 0,09 & & 0,08 & 0,09 & 0,09 & & 0,08 & 0,09 & 0,09 & 0,09 \\
\hline & 8,0 & Jan-93 & Dec-97 & MTAR* & $-0,09$ & 0,24 & & 0,13 & 0,1 & 0,24 & 0 & 0,13 & 0, & 0,23 & & 0,1 & & 0,24 & & 0,13 & 0,18 & 0,24 & 0,22 \\
\hline & 8,0 & Jan & Dec-98 & TAR* & 0,31 & & 0, & 0, & 0, & 0 & & 0,1 & 0, & 0,2 & & 0 , & & & & 0 , & & 0,25 & 0,18 \\
\hline & $\begin{array}{l}8,04 \\
8,04\end{array}$ & Jan-95 & Dec-99 & MTAR* & $\begin{array}{r}0,01 \\
-2,13\end{array}$ & 0, & 0, & 0,15 & $\begin{array}{l}0,21 \\
0,25\end{array}$ & $\begin{array}{l}0,23 \\
0,37\end{array}$ & 0, & 0,15 & $\begin{array}{l}0,21 \\
0,25\end{array}$ & $\begin{array}{l}0,23 \\
0,37\end{array}$ & 0, & 0, & 0, & 0,37 & & 0,15 & $\begin{array}{l}0,21 \\
0,25\end{array}$ & $\begin{array}{l}0,23 \\
0,37\end{array}$ & $\begin{array}{l}0,40 \\
0,40\end{array}$ \\
\hline & 8,04 & Jan- & Dec- & MTAR* & $-1,18$ & 0,26 & & 0,16 & 0,27 & 0,43 & 0, & 0,1 & 0,2 & 0,43 & & 0,16 & 0,2 & 0,43 & & 0,16 & 0,27 & 0,43 & 0,48 \\
\hline & 8,04 & Jan-97 & Dec-01 & MTAR* & 0,80 & 0,26 & 0,49 & 0,17 & 0,28 & 0,42 & 0,47 & 0,17 & 0,28 & 0,42 & 0,4 & 0,17 & 0,28 & 0,42 & 0,4 & 0,17 & 0,28 & 0,42 & 0,47 \\
\hline & 8,8 & Jan-98 & Dec-02 & & 0,37 & & & 0,5 & 0 & & & & 0,8 & & & & & & & & & & 0,70 \\
\hline & 8,0 & Jan & Dec- & MTAR* & 0,09 & 0, & 0, & 0, & 0,6 & & 0, & & 0, & & & & & & & 0,4 & & & 0,59 \\
\hline Estonia & $\begin{array}{l}8,04 \\
8,01\end{array}$ & & & SYM & & & & 0 & 0 & 0 & & 0, & 0, & 0,5 & & & & 0,4 & & 0,4 & $\begin{array}{l}0,00 \\
0,41\end{array}$ & 0,42 & $\begin{array}{l}0,42 \\
0,42\end{array}$ \\
\hline & 8,01 & Jan-97 & Dec-01 & SYM & & 0,43 & 0,45 & 0,41 & 0,43 & 0,44 & 0,45 & 0,41 & 0,43 & 0,44 & 0,4 & 0,41 & 0,43 & 0,44 & 0,4 & 0,41 & 0,43 & 0,44 & 0,45 \\
\hline & 8,01 & Jan-98 & Dec-02 & SYM & & 0,20 & 0,42 & 0,28 & 0,37 & 0,41 & 0,4 & 0,28 & 0,37 & 0.41 & & 0,28 & 0,37 & 0,41 & & 0,28 & 0,37 & 0,41 & 0.42 \\
\hline & 8,01 & Jan-99 & Dec-03 & SY & & & & & 0 & & & & 0 & & & & & & & & & & \\
\hline & $\begin{array}{l}8,01 \\
8,02\end{array}$ & & & S1 & & & 0 , & 0, & 0, & 0, & 0, & 0, & 0, & 0, & 0 & 0, & & & & 0 & 0, & 0,24 & 0,24 \\
\hline & 8,02 & Jan-97 & Dec- & STD & & 0, & 0,2 & 0,25 & 0,2 & 0,25 & 0,2 & 0,2 & 0,2 & 0,25 & 0,2 & 0,25 & 0,25 & 0,25 & 0,2 & 0,25 & 0,25 & 0,25 & 0,25 \\
\hline & 8,02 & Jan-98 & Dec-02 & STD & & 0,17 & 0,23 & 0,21 & 0,23 & 0,23 & 0,23 & 0,21 & 0,23 & 0,23 & 0,23 & 0,21 & 0,23 & 0,23 & 0,23 & 0,21 & 0,23 & 0,23 & 0,23 \\
\hline & 8,02 & Jan-99 & Dec-03 & STD & & 0,28 & 0,38 & 0,35 & 0,3 & 0,38 & 0 & 0,3 & 0,3 & 0,3 & 0 & 0,35 & 0,38 & 0,38 & 0 , & 0,35 & 0,38 & 0,38 & 0,38 \\
\hline & 8,03 & Jan-96 & Dec-00 & SYM & & & & 0,5 & 0 & 0,5 & & & 0,5 & 0 & & & & & & 0,56 & & & 0,55 \\
\hline & $\begin{array}{l}8,03 \\
8,03\end{array}$ & Jan-97 & Dec-01 & SYM & & 0,54 & 0,5 & $\begin{array}{l}0,50 \\
0,57\end{array}$ & $\begin{array}{l}0,50 \\
0,57\end{array}$ & 0,56 & & 0,57 & 0,57 & 0,56 & 0,56 & 0,57 & 0,57 & 0,56 & 0,56 & $\begin{array}{l}0,50 \\
0,57\end{array}$ & $\begin{array}{l}0,50 \\
0,57\end{array}$ & 0,56 & 0,56 \\
\hline & 8003 & J & Dec-0? & SYM & & 035 & 050 & 042 & 046 & 048 & 050 & 047 & 046 & 048 & 00 & 04 & 046 & 048 & 050 & 042 & 046 & 048 & 050 \\
\hline
\end{tabular}


Table B6: 5-year rolling multipliers of the optimal pass-through model

\begin{tabular}{|c|c|c|c|c|c|c|c|c|c|c|c|c|c|c|c|c|c|c|c|c|c|c|c|}
\hline \multirow{2}{*}{ country } & \multirow[b]{2}{*}{ rate } & \multicolumn{2}{|c|}{ sample period } & \multirow{2}{*}{$\begin{array}{r}\text { pass-through } \\
\text { model }\end{array}$} & \multirow{2}{*}{\multicolumn{3}{|c|}{$\begin{array}{l}\text { thres- impact long-run } \\
\text { hold multiplier multiplier }\end{array}$}} & \multicolumn{4}{|c|}{ multipliers for +1\% shock } & & ipliers fo & r $-1 \%$ sho & & multip & Dhers for - & $+0.25 \% \mathrm{~s}$ & Shock & multtr & pliers for & $-0.25 \% \mathrm{~s}$ & \\
\hline & & start & end & & & & & $1 \mathrm{mth}$ & $3 \mathrm{mths}$ & $6 \mathrm{mths}$ & $12 \mathrm{mths}$ & $1 \mathrm{mth}$ & $3 \mathrm{mths}$ & $6 \mathrm{mths}$ & $12 \mathrm{mths}$ & $1 \mathrm{mth}$ & $3 \mathrm{mths}$ & $6 \mathrm{mths}$ & $12 \mathrm{mths}$ & $1 \mathrm{mth}$ & $3 \mathrm{mths}$ & $6 \mathrm{mths}$ & $12 \mathrm{mths}$ \\
\hline & 8,03 & $\begin{array}{l}\text { Jan-99 } \\
\end{array}$ & Dec-03 & & & 0,49 & 0,46 & 0,46 & 0,46 & 0,46 & 0,46 & 0,46 & 0,46 & 0,46 & 0,46 & 0,46 & 0,46 & 0,46 & 0,46 & 0,46 & 0,46 & 0,46 & 0,46 \\
\hline & 8,04 & Jan-97 & Dec-01 & SYM & & 0,30 & 0,59 & 0,38 & 0,45 & 0,50 & 0,56 & 0,38 & 0,45 & 0,50 & 0,56 & 0,38 & 0,45 & 0,50 & 0,56 & 0,38 & 0,45 & 0,50 & 0,56 \\
\hline & 8,04 & Jan-98 & Dec-02 & SYM & & 0,25 & 0,63 & 0,36 & 0,47 & 0,55 & 0,61 & 0,36 & 0,47 & 0,55 & 0,61 & 0,36 & 0,47 & 0,55 & 0,61 & 0,36 & 0,47 & 0,55 & 0,61 \\
\hline & 8,04 & Jan-99 & Dec-03 & SYM & & 0,43 & 0,77 & 0,63 & 0,75 & 0,77 & 0,77 & 0,63 & 0,75 & 0,77 & 0,77 & 0,63 & 0,75 & 0,77 & 0,77 & 0,63 & 0,75 & 0,77 & 0,77 \\
\hline & 8,05 & Jan-96 & Dec-00 & & & & & & & & & & & & & & & & & & & & \\
\hline & 8,05 & Jan-97 & Dec-01 & & & & & & & & & & & & & & & & & & & & \\
\hline & 8,05 & Jan-98 & Dec-02 & MTAR* & 0,30 & 0,03 & 0,70 & 0,29 & 0,30 & 0,39 & 0,51 & 0,29 & 0,34 & 0,47 & 0,59 & 0,29 & 0,30 & 0,39 & 0,51 & 0,29 & 0,30 & 0,39 & 0,51 \\
\hline & 8,05 & Jan-99 & Dec-03 & MTAR* & 0,19 & 0,38 & 0,88 & 0,45 & 0,73 & 0,78 & 0,84 & 0,45 & 0,70 & 0,75 & 0,81 & 0,45 & 0,73 & 0,79 & 0,85 & 0,45 & 0,73 & 0,79 & 0,85 \\
\hline & 8,06 & Jan-96 & Dec-00 & MTAR* & $-1,86$ & 0,33 & 0,18 & $-0,07$ & 0,11 & 0,02 & 0,13 & $-0,07$ & 0,11 & 0,02 & 0,13 & $-0,07$ & 0,11 & 0,02 & 0,13 & $-0,07$ & 0,11 & 0,02 & 0,13 \\
\hline & 8,06 & Jan-97 & Dec-01 & & & & & & & & & & & & & & & & & & & & \\
\hline & 8,06 & $\begin{array}{l}\text { Jan-98 } \\
J a n-99\end{array}$ & Dec-02 & MTAR* & $-0,50$ & 0,17 & 0,42 & $-0,06$ & 0,20 & 0,27 & 0,33 & $-0,06$ & 0,20 & 0,27 & 0,33 & $-0,06$ & 0,20 & 0,27 & 0,33 & $-0,06$ & 0,20 & 0,27 & 0,33 \\
\hline Hungary & $\begin{array}{l}8,00 \\
8.01\end{array}$ & $\begin{array}{l}\text { Jana-99 } \\
\text { Jan-95 }\end{array}$ & $\begin{array}{l}\text { Dec-03 } \\
\text { Dec-99 }\end{array}$ & MTAR* & 0.13 & 0.29 & 0.82 & 0.22 & 1.01 & 1.17 & 0.65 & 020 & 0.87 & 1.09 & 069 & 022 & 101 & 114 & 066 & 022 & 087 & 109 & 068 \\
\hline & 8,01 & Jan-96 & Dec-00 & MTAR* & $-0,32$ & 0,33 & 0,85 & 0,47 & 0,93 & 0,94 & 0,86 & 0,47 & 0,92 & 0,93 & 0,86 & 0,47 & 0,92 & 0,93 & $\begin{array}{l}0,00 \\
0,86\end{array}$ & $\begin{array}{l}0,22 \\
0.47\end{array}$ & 0,92 & 0,93 & 0,86 \\
\hline & 8,01 & Jan-97 & Dec-01 & MTAR* & 0,03 & 0,34 & 0,84 & 0,50 & 0,86 & 0,92 & 0,88 & 0,50 & 0,84 & 0,90 & 0,87 & 0,50 & 0,86 & 0,91 & 0,87 & 0,50 & 0,84 & 0,90 & 0,88 \\
\hline & 8,01 & Jan-98 & Dec-02 & MTAR* & 0,05 & 0,35 & 0,84 & 0,56 & 0,87 & 0,87 & 0,87 & 0,56 & 0,87 & 0,88 & 0,88 & 0,56 & 0,87 & 0,87 & 0,87 & 0,56 & 0,87 & 0,87 & 0,87 \\
\hline & 8,01 & Jan-99 & Dec-03 & MTAR* & 0,52 & 0,45 & 0,78 & 0,62 & 0,83 & 0,86 & 0,77 & 0,62 & 0,83 & 0,86 & 0,77 & 0,62 & 0,83 & 0,86 & 0,77 & 0,62 & 0,83 & 0,86 & 0,77 \\
\hline & $\begin{array}{l}8,01 \\
8,02\end{array}$ & Jan-95 & Dec-99 & SYM & $0, J 2$ & 0,23 & 0,98 & 0,60 & 0,96 & $\begin{array}{l}0,00 \\
0,99\end{array}$ & 0,98 & 0,60 & $\begin{array}{l}0,03 \\
0,96\end{array}$ & $\begin{array}{l}0, \infty 0 \\
0,99\end{array}$ & 0,98 & 0,60 & $\begin{array}{l}0,036 \\
0,96\end{array}$ & 0,99 & 0,98 & 0,60 & 0,96 & 0,99 & 0,98 \\
\hline & 8,02 & Jan-96 & Dec-00 & SYM & & 0,28 & 0,90 & 0,61 & 0,89 & 0,91 & 0,90 & 0,61 & 0,89 & 0,91 & 0,90 & 0,61 & 0,89 & 0,91 & 0,90 & 0,61 & 0,89 & 0,91 & 0,90 \\
\hline & $\begin{array}{l}8,02 \\
8,02\end{array}$ & Jan-97 & Dec-01 & SYM & & 0,32 & 0,87 & 0,54 & 0,75 & 0,84 & 0,87 & 0,54 & 0,75 & 0,84 & 0,87 & 0,54 & 0,75 & 0,84 & 0,87 & 0,54 & 0,75 & 0,84 & 0,87 \\
\hline & 8,02 & Jan-98 & Dec-02 & SYM & & 0,26 & 0,79 & 0,51 & 0,72 & 0,78 & 0,79 & 0,51 & 0,72 & 0,78 & 0,79 & 0,51 & 0,72 & 0,78 & 0,79 & 0,51 & 0,72 & 0,78 & 0,79 \\
\hline & 8,02 & Jan-99 & Dec-03 & SYM & & 0,29 & 0,76 & 0,42 & 0,61 & 0,71 & 0,76 & 0,42 & 0,61 & 0,71 & 0,76 & 0,42 & 0,61 & 0,71 & 0,76 & 0,42 & 0,61 & 0,71 & 0,76 \\
\hline Latvia & 8,01 & Jan-99 & Dec-03 & SYM & & 0,86 & 0,77 & 0,70 & 0,76 & 0,77 & 0,77 & 0,70 & 0,76 & 0,77 & 0,77 & 0,70 & 0,76 & 0,77 & 0,77 & 0,70 & 0,76 & 0,77 & 0,77 \\
\hline & 8,02 & Jan-99 & Dec-03 & SYM & & 0,21 & 0,56 & 0,30 & 0,42 & 0,50 & 0,55 & 0,30 & 0,42 & 0,50 & 0,55 & 0,30 & 0,42 & 0,50 & 0,55 & 0,30 & 0,42 & 0,50 & 0,55 \\
\hline & 8,03 & Jan-99 & Dec-03 & SYM & & 0,01 & 0,54 & 0,13 & 0,26 & 0,38 & 0,49 & 0,13 & 0,26 & 0,38 & 0,49 & 0,13 & 0,26 & 0,38 & 0,49 & 0,13 & 0,26 & 0,38 & 0,49 \\
\hline & 8,04 & Jan-99 & Dec-03 & SYM & & 0,00 & 0,38 & 0,02 & 0,04 & 0,08 & 0,13 & 0,02 & 0,04 & 0,08 & 0,13 & 0,02 & 0,04 & 0,08 & 0,13 & 0,02 & 0,04 & 0,08 & 0,13 \\
\hline & 8,05 & Jan-99 & Dec-03 & SYM & & 0,52 & 0,68 & 0,45 & 0,55 & 0,62 & 0,67 & 0,45 & 0,55 & 0,62 & 0,67 & 0,45 & 0,55 & 0,62 & 0,67 & 0,45 & 0,55 & 0,62 & 0,67 \\
\hline & 8,06 & Jan-99 & Dec-03 & SYM & & 0,02 & 0,30 & 0,04 & 0,08 & 0,12 & 0,18 & 0,04 & 0,08 & 0,12 & 0,18 & 0,04 & 0,08 & 0,12 & 0,18 & & 0,08 & 0,12 & 0,18 \\
\hline & 8,07 & Jan-99 & Dec-03 & SYM & & 0,87 & 0,78 & 0,70 & 0,77 & 0,78 & 0,78 & 0,70 & 0,77 & 0,78 & 0,78 & 0,70 & 0,77 & 0,78 & 0,78 & 0,70 & 0,77 & 0,78 & 0,78 \\
\hline & 8,08 & Jan-99 & Dec-03 & SYM & & 0,27 & 0,64 & 0,39 & 0,52 & 0,60 & 0,63 & 0,39 & 0,52 & 0,60 & 0,63 & 0,39 & 0,52 & 0,60 & 0,63 & 0,39 & 0,52 & 0,60 & 0,63 \\
\hline & 8,09 & Jan-99 & Dec-03 & SYM & & 0,11 & 0,64 & 0,28 & 0,45 & 0,57 & 0,63 & 0,28 & 0,45 & 0,57 & 0,63 & 0,28 & 0,45 & 0,57 & 0,63 & 0,28 & 0,45 & 0,57 & 0,63 \\
\hline & 8,1 & Jan-99 & Dec-03 & SYM & & $\begin{array}{r}0,11 \\
-0,01\end{array}$ & 0,34 & $\begin{array}{l}0,20 \\
0,12\end{array}$ & 0,23 & 0,30 & 0,34 & $\begin{array}{l}0,20 \\
0,12\end{array}$ & $\begin{array}{l}0,43 \\
0,23\end{array}$ & 0,30 & 0,34 & $\begin{array}{l}, 20 \\
0,12\end{array}$ & $\begin{array}{l}0,43 \\
0,23\end{array}$ & 0,30 & 0,34 & $\begin{array}{l}, 20 \\
0,12\end{array}$ & 0,23 & 0,30 & 0,34 \\
\hline & 8,11 & Jan-99 & Dec-03 & SYM & & 0,61 & 0,73 & 0,55 & 0,65 & 0,70 & 0,73 & 0,55 & 0,65 & 0,70 & 0,73 & 0,55 & 0,65 & 0,70 & 0,7 & 0,55 & 0,65 & 0,70 & 0,73 \\
\hline & $\begin{array}{l}8,11 \\
8,12\end{array}$ & Jan-99 & Dec-03 & SYM & & 0,17 & 0,07 & 0,07 & 0,09 & 0,09 & 0,08 & 0,07 & 0,09 & 0,09 & 0,08 & 0,07 & 0,09 & 0,09 & 0,08 & 0,07 & 0,09 & 0,09 & 0,08 \\
\hline & 8,13 & Jan-99 & Dec-03 & MTAR* & 0,29 & 0,29 & 0,47 & 0,46 & 0,44 & 0,45 & 0,46 & 0,46 & 0,44 & 0,45 & 0,46 & 0,46 & 0,44 & 0,45 & 0,46 & 0,46 & 0,44 & 0,45 & 0,46 \\
\hline & 8,14 & Jan-99 & Dec-03 & SYM & & $-0,01$ & 0,32 & 0,03 & 0,08 & 0,14 & 0,22 & 0,03 & 0,08 & 0,14 & 0,22 & 0,03 & 0,08 & 0,14 & 0,22 & 0,03 & 0,08 & 0,14 & 0,22 \\
\hline & 8,15 & Jan-99 & Dec-03 & STD & & 0,00 & 0,00 & 0,00 & 0,00 & 0,00 & 0,00 & 0,00 & 0,00 & 0,00 & 0,00 & 0,00 & 0,00 & 0,00 & & 0, & 0,00 & 0,00 & 0,00 \\
\hline & 8,16 & Jan-99 & Dec-03 & STD & & $-0,01$ & $-0,01$ & $-0,01$ & $-0,01$ & $-0,01$ & $-0,01$ & $-0,01$ & $-0,01$ & $-0,01$ & $-0,01$ & $-0,01$ & $-0,01$ & $-0,01$ & $-0,0$ & $-0,01$ & $-0,01$ & $-0,01$ & $-0,01$ \\
\hline & $\begin{array}{l}8,17 \\
8,17\end{array}$ & Jan-99 & Dec-03 & $\begin{array}{l}\text { STD } \\
\text { ST }\end{array}$ & & $\begin{array}{r}-0,01 \\
0,05\end{array}$ & 0,05 & 0,05 & 0,05 & 0,05 & $\begin{array}{r}-0,01 \\
0,05\end{array}$ & 0,05 & $\begin{array}{r}-, 01 \\
0,05\end{array}$ & $\begin{array}{r}-, 01 \\
0,05\end{array}$ & $\begin{array}{r}-0,01 \\
0,05\end{array}$ & $\begin{array}{r}-, 01 \\
0,05\end{array}$ & $\begin{array}{r}-0,01 \\
0,05\end{array}$ & 0,05 & 0,0 & 0,05 & 0,05 & 0,05 & $\begin{array}{r}-0,01 \\
0,05\end{array}$ \\
\hline & 8,18 & Jan-99 & Dec-03 & STD & & $-0,04$ & $-0,03$ & $-0,03$ & $-0,03$ & $-0,03$ & $-0,03$ & $-0,03$ & $-0,03$ & $-0,03$ & $-0,03$ & $-0,03$ & $-0,03$ & $-0,03$ & $-0,03$ & $-0,03$ & $-0,03$ & $-0,03$ & $-0,03$ \\
\hline Lithuania & 8,01 & Jan-94 & Dec-98 & MTAR* & 2,59 & 0,81 & 0,81 & 1,08 & 0,83 & 0,80 & 0,81 & 1,08 & 0,83 & 0,80 & 0,81 & 1,08 & 0,83 & 0,80 & 0,81 & 1,08 & 0,83 & 0,80 & 0,81 \\
\hline & 8,0 & Jan-95 & Dec-9 & MTAR* & $-1,79$ & 0,2 & 0, & 0,5 & & & 0,6 & 0,5 & & 0, & 0 & 0,5 & 0, & 0,67 & 0, & 0 & & 0,67 & 0,68 \\
\hline & 8,01 & Jan-96 & Dec-00 & MTAR* & 1,39 & 0,17 & 0,66 & 0,36 & 0,48 & 0,58 & 0,64 & 0,36 & 0,48 & 0,58 & 0,64 & 0,36 & 0,48 & 0,58 & 0,64 & 0,36 & 0,48 & 0,58 & 0,64 \\
\hline & 8,01 & Jan-97 & Dec-01 & MTAR* & $-0,35$ & 0,1 & 0,53 & 0,29 & 0,3 & 0,44 & 0,50 & 0,29 & 0,3 & 0,45 & 0,50 & 0,29 & 0 & 0,45 & 0 & 0,29 & 0,36 & 0,45 & 0,50 \\
\hline & 8,01 & Jan-98 & Dec-02 & MTAR* & 0,25 & 0,08 & 0,47 & 0,31 & 0,38 & 0,42 & 0,46 & 0,31 & 0,38 & 0,43 & 0,4 & 0,31 & 0,38 & 0,42 & 0,46 & 0,31 & 0,38 & 0,42 & 0,46 \\
\hline & $\begin{array}{l}8,01 \\
8,01\end{array}$ & Jan-99 & Dec-03 & MTAR* & $-0,54$ & 0,07 & 0,41 & 0,32 & $\begin{array}{l}0,28 \\
0,28\end{array}$ & $\begin{array}{l}0,42 \\
0,34\end{array}$ & $\begin{array}{l}0,40 \\
0,38\end{array}$ & 0,32 & 0,28 & 0,34 & 0,3 & 0,32 & $\begin{array}{l}0,28 \\
0,28\end{array}$ & 0,34 & $\begin{array}{l}0,40 \\
0,38\end{array}$ & 0,32 & 0,28 & 0,34 & $\begin{array}{l}0,38 \\
0,38\end{array}$ \\
\hline & 8,02 & Jan-99 & Dec-03 & SYM & & 0,03 & & & & & & & & & & & & & & & & 0,19 & 0,31 \\
\hline & 8,0 & Jan-94 & Dec- & SY & & 0,2 & 0, & 0,4 & & & & 0,4 & & 0, & 0, & 0 & 0, & 0, & & 0, & 56 & 0,65 & 0,64 \\
\hline & 8,03 & Jan-95 & Dec & & & $-0,0$ & 0 , & 0,07 & & & & & & 0 & 0 & 0,07 & 0, & 0,3 & 0,4 & 0,07 & 0,20 & 0,34 & 0,47 \\
\hline & 8,03 & Jan-96 & Dec-00 & SYM & & 0,08 & 0,41 & 0,14 & 0,24 & 0,32 & 0,38 & 0,14 & 0,24 & 0,32 & 0,38 & 0,14 & 0,24 & 0,32 & 0,38 & 0,14 & 0,24 & 0,32 & 0,38 \\
\hline & 8,03 & & Dec-0 & SY & & & & 0,04 & 0,09 & 0,16 & 0,24 & & 0,09 & & & & & & & & 0,09 & 0,16 & 0,24 \\
\hline & 8,0 & Jan- & Dec- & SY & & 0 , & & 0, & & & & & 0, & & & & & & & & & 0,08 & 0,15 \\
\hline & 8,03 & & & SY & & & & 0, & & & 0, & & & & 0, & 0,01 & 0,07 & 0,16 & 0,3 & 0,01 & 0,07 & 0,16 & 0,31 \\
\hline & 8,04 & Jan-99 & Dec-03 & SYM & & $-0,02$ & 0,72 & 0,00 & 0,06 & 0,16 & 0,33 & 0,00 & 0,06 & 0,16 & 0,33 & 0,00 & 0,06 & 0,16 & 0,33 & 0,00 & 0,06 & 0,16 & 0,33 \\
\hline & 8,05 & Jan-94 & Dec-98 & SYM & & 0,25 & 0,69 & 0,51 & 0,67 & 0,69 & 0,69 & 0,51 & 0,67 & 0,69 & 0,69 & 0,51 & 0,67 & 0,69 & 0,69 & 0,51 & 0,67 & 0,69 & 0,69 \\
\hline & 8,05 & Jan-95 & Dec-99 & SY & & 0,10 & 0,59 & 0,2 & 0, & 0,55 & 0,5 & 0,29 & 0,45 & 0 & 0 & 0,29 & 0,45 & 0,55 & 0, & 0,29 & 5 & 0,55 & 0,59 \\
\hline & & & & & & & & & & & & & & & & & & & & & & 43 & 0,46 \\
\hline & 8,05 & Jan-97 & Dec-01 & SYM & & 0,01 & 0,32 & 0,04 & 0,09 & 0,16 & 0,24 & 0,04 & 0,09 & 0,16 & 0,24 & 0,04 & 0,09 & 0,16 & 0,24 & 0,04 & 0,09 & 0,16 & 0,24 \\
\hline & 8,05 & Jan-98 & Dec-02 & SYM & & $-0,02$ & 0,49 & $-0,01$ & 0,04 & 0,10 & 0,19 & $-0,01$ & 0,04 & 0,10 & 0,19 & $-0,01$ & 0,04 & 0,10 & 0,19 & $-0,01$ & 0,04 & 0,10 & 0,19 \\
\hline & 8,05 & Jan-99 & Dec-03 & SYM & & $\begin{array}{r}-0,02 \\
0,00\end{array}$ & 0,75 & 0,02 & 0,08 & 0,16 & 0,29 & $\begin{array}{r}0,01 \\
0,02\end{array}$ & 0,08 & 0,16 & 0,29 & 0,02 & 0,08 & 0,16 & 0,29 & 0,02 & 0,08 & 0,16 & 0,29 \\
\hline & 8,06 & Jan-99 & Dec-03 & SYM & & 0,00 & 0,74 & 0,02 & 0,08 & 0,17 & 0,31 & 0,02 & 0,08 & 0,17 & 0,31 & 0,02 & 0,08 & 0,17 & 0,31 & 0,02 & 0,08 & 0,17 & 0.31 \\
\hline
\end{tabular}


Table B6: 5-year rolling multipliers of the optimal pass-through model

\begin{tabular}{|c|c|c|c|c|c|c|c|c|c|c|c|c|c|c|c|c|c|c|c|c|c|c|c|}
\hline \multirow{3}{*}{ country } & \multirow[b]{2}{*}{ rate } & & \multirow{2}{*}{$\begin{array}{r}\text { pass-through } \\
\text { model }\end{array}$} & \multirow{2}{*}{$\begin{array}{l}\text { thres- } \\
\text { hold } \mathrm{n}\end{array}$} & \multirow{2}{*}{\multicolumn{2}{|c|}{$\begin{array}{l}\text { impact long-run } \\
\text { ultiplier multiplier }\end{array}$}} & & & & & & & & & & & & & & & & \\
\hline & & start & end & & & & & $1 \mathrm{mth}$ & $3 \mathrm{mths}$ & $6 \mathrm{mths}$ & $12 \mathrm{mths}$ & $1 \mathrm{mth}$ & $3 \mathrm{mths}$ & $6 \mathrm{mths}$ & $12 \mathrm{mths}$ & $1 \mathrm{mth}$ & $3 \mathrm{mths}$ & 6 mths & $12 \mathrm{mths}$ & $1 \mathrm{mth}$ & 3 mths & $6 \mathrm{mths}$ & $12 \mathrm{mths}$ \\
\hline & 807 & Tan-94 & Dec-98 & SYM & & 014 & 069 & 051 & 072 & 069 & 069 & 051 & 072 & 069 & 069 & 051 & 072 & 069 & 069 & 051 & 072 & 069 & 069 \\
\hline & 8,07 & Jan-95 & Dec-99 & SYM & & 0,13 & 0.48 & 0,32 & 0,43 & 0,47 & 0.48 & 0,32 & 0,43 & 0,47 & 0.48 & 0,32 & 0.43 & 0.47 & 0.48 & 0,32 & 0,43 & 0,47 & 0.48 \\
\hline & $\begin{array}{l}8,07 \\
8,07\end{array}$ & Jan-96 96 & Dec-00 & SYM & & 0,17 & 0,37 & 0,34 & 0,37 & 0,37 & $\begin{array}{l}0,40 \\
0,37\end{array}$ & 0,34 & $\begin{array}{l}0,43 \\
0,37\end{array}$ & 0,37 & $\begin{array}{l}0,40 \\
0,37\end{array}$ & 0,34 & 0,37 & 0,37 & $\begin{array}{l}0,40 \\
0,37\end{array}$ & 0,34 & 0,37 & 0,37 & 0,37 \\
\hline & 8,07 & Jan-97 & Dec-01 & SYM & & 0,07 & 0,33 & 0,10 & 0,16 & 0,22 & 0,29 & 0,10 & 0,16 & 0,22 & 0,29 & 0,10 & 0,16 & 0,22 & 0,29 & 0,10 & 0,16 & 0,22 & 0,29 \\
\hline & 8,07 & Jan-98 & Dec-02 & SYM & & 0,04 & 0,50 & 0,05 & 0,08 & 0,12 & 0,18 & 0,05 & 0,08 & 0,12 & 0,18 & 0,05 & 0,08 & 0,12 & 0,18 & 0,05 & 0,08 & 0,12 & 0,18 \\
\hline & $\begin{array}{l}8,07 \\
8,07\end{array}$ & Jan-99 & $\begin{array}{l}\text { Dec-02 } \\
\text { Dec-03 }\end{array}$ & SYM & & $\begin{array}{l}0,04 \\
0,00\end{array}$ & 0,75 & 0,03 & $\begin{array}{l}0,08 \\
0,08\end{array}$ & $\begin{array}{l}0,12 \\
0,17\end{array}$ & $\begin{array}{l}0,18 \\
0,30\end{array}$ & 0,03 & $\begin{array}{l}0,08 \\
0,08\end{array}$ & $\begin{array}{l}0,12 \\
0,17\end{array}$ & $\begin{array}{l}0,318 \\
0,30\end{array}$ & 0,03 & $\begin{array}{l}0,08 \\
0,08\end{array}$ & $\begin{array}{l}0,12 \\
0,17\end{array}$ & $\begin{array}{l}0,18 \\
0,30\end{array}$ & 0,03 & $\begin{array}{l}0,08 \\
0,08\end{array}$ & $\begin{array}{l}0,12 \\
0,17\end{array}$ & $\begin{array}{l}0,18 \\
0,30\end{array}$ \\
\hline & 8,08 & Jan-99 & Dec-03 & SYM & & 0,00 & 0,75 & 0,02 & 0,09 & 0,19 & 0,34 & 0,02 & 0,09 & 0,19 & 0,34 & 0,02 & 0,09 & 0,19 & 0,34 & 0,02 & 0,09 & 0,19 & 0,34 \\
\hline & $\begin{array}{l}8,00 \\
8,09\end{array}$ & Jan-94 & Dec-98 & SYM & & 0,22 & 0,52 & 0,42 & 0,51 & $\begin{array}{l}0,19 \\
0,52\end{array}$ & $\begin{array}{l}0,54 \\
0,52\end{array}$ & $\begin{array}{l}0,02 \\
0,42\end{array}$ & $\begin{array}{l}0,51 \\
0,51\end{array}$ & $\begin{array}{l}0,19 \\
0,52\end{array}$ & 0,52 & $\begin{array}{l}0,02 \\
0,42\end{array}$ & 0,51 & $\begin{array}{l}0,19 \\
0,52\end{array}$ & $\begin{array}{l}0,54 \\
0,52\end{array}$ & 0,42 & 0,51 & $\begin{array}{l}0,19 \\
0,52\end{array}$ & 0,52 \\
\hline & 8,09 & Jan-95 & Dec-99 & SYM & & $-0,04$ & 0,46 & 0,25 & 0,40 & 0,45 & 0,46 & 0,25 & 0,40 & 0,45 & 0,46 & 0,25 & 0,40 & 0,45 & 0,46 & 0,25 & 0,40 & 0,45 & 0,46 \\
\hline & 8,09 & Jan-96 & Dec-00 & SYM & & 0,02 & 0,14 & 0,08 & 0,12 & 0,14 & 0,14 & 0,08 & 0,12 & 0,14 & 0,14 & 0,08 & 0,12 & 0,14 & 0,14 & 0,08 & 0,12 & 0,14 & 0,14 \\
\hline & 8,09 & Jan-97 & Dec-01 & SYM & & 0,00 & 0,23 & 0,04 & 0,09 & 0,14 & 0,19 & 0,04 & 0,09 & 0,14 & 0,19 & 0,04 & 0,09 & 0,14 & 0,19 & 0,04 & 0,09 & 0,14 & 0,19 \\
\hline & 8,09 & Jan-98 & Dec-02 & SYM & & 0,03 & 0,39 & 0,05 & 0,08 & 0,12 & 0,20 & 0,05 & 0,08 & 0,12 & 0,20 & 0,05 & 0,08 & 0,12 & 0,20 & 0,05 & 0,08 & 0,12 & 0,20 \\
\hline & 8,09 & Jan-99 & Dec-03 & SYM & & 0,02 & 0,67 & 0,06 & 0,12 & 0,20 & 0,33 & 0,06 & 0,12 & 0,20 & 0,33 & 0,06 & 0,12 & 0,20 & 0,33 & 0,06 & 0,12 & 0,20 & 0,33 \\
\hline & 8,1 & Jan-99 & Dec-03 & STD & & 0,02 & 0,03 & 0,03 & 0,03 & 0,03 & 0,03 & 0,03 & 0,03 & 0,03 & 0,03 & 0,03 & 0,03 & 0,03 & 0,03 & 0,03 & 0,03 & 0,03 & 0,03 \\
\hline & $\begin{array}{l}0,1 \\
8,11\end{array}$ & Jan-97 & Dec-01 & MTAR* & $-1,59$ & $\begin{array}{r}, 0,10 \\
-0,10\end{array}$ & 0,17 & $\begin{array}{r}, 0,02 \\
-0,02\end{array}$ & 0,11 & 0,20 & 0,17 & $\begin{array}{r}-0,02 \\
-0,02\end{array}$ & 0,11 & 0,20 & 0,17 & $\begin{array}{r}-0,02 \\
-0,02\end{array}$ & 0,11 & 0,20 & 0,17 & $\begin{array}{r}-0,02 \\
-0,02\end{array}$ & 0,11 & 0,20 & 0,17 \\
\hline & 8,11 & Jan-98 & Dec-02 & MTAR* & $-0,96$ & 0,00 & 0,38 & 0,09 & 0,15 & 0,11 & 0,26 & 0,09 & 0,15 & 0,11 & 0,26 & 0,09 & 0,15 & 0,11 & 0,26 & 0,09 & 0,15 & 0,11 & 0,26 \\
\hline & 8,11 & Jan-99 & Dec-03 & MTAR* & $-1,62$ & 0,06 & 0,60 & 0,11 & 0,19 & 0,21 & 0,43 & 0,11 & 0,19 & 0,21 & 0,43 & 0,11 & 0,19 & 0,21 & 0,43 & 0,11 & 0,19 & 0,21 & 0,43 \\
\hline & 8,12 & Jan-99 & Dec-03 & STD & & $-0,03$ & $-0,03$ & $-0,03$ & $-0,03$ & $-0,03$ & $-0,03$ & $-0,03$ & $-0,03$ & $-0,03$ & $-0,03$ & $-0,03$ & $-0,03$ & $-0,03$ & $-0,03$ & $-0,03$ & $-0,03$ & $-0,03$ & $-0,03$ \\
\hline & 8,13 & Jan-94 & Dec-98 & MTAR* & 0,58 & 0,39 & 0,67 & 0,74 & 0,63 & 0,67 & 0,67 & 0,74 & 0,63 & 0,67 & 0,67 & 0,74 & 0,63 & 0,67 & 0,67 & 0,74 & 0,63 & 0,67 & 0,67 \\
\hline & 8,13 & Jan-95 & Dec-99 & MTAR* & $-1,60$ & 0,04 & 0,62 & 0,19 & 0,31 & 0,48 & 0,63 & 0.19 & 0,31 & 0,48 & 0,63 & 0,19 & 0,31 & 0.48 & 0,63 & 0,19 & 0,31 & 0,48 & 0,63 \\
\hline & 8,13 & Jan-96 & Dec-00 & MTAR* & $-0,94$ & 0,10 & 0,54 & 0,21 & 0,39 & 0,47 & 0,52 & 0,21 & 0,39 & 0,47 & 0,52 & 0,21 & 0,39 & 0,47 & 0,52 & 0,21 & 0,39 & 0,47 & 0,52 \\
\hline & 8,13 & Jan-97 & Dec-01 & MTAR* & $-0,91$ & 0,10 & 0,53 & 0,21 & 0,32 & 0,37 & 0,44 & 0,21 & 0,32 & 0,37 & 0,44 & 0,21 & 0,32 & 0,37 & 0,44 & 0,21 & 0,32 & 0,37 & 0,44 \\
\hline & 8,13 & Jan-98 & Dec-02 & MTAR* & $\begin{array}{l}-0,69 \\
-\end{array}$ & 0,08 & 0,57 & 0,21 & 0,31 & 0,37 & 0,44 & 0,21 & 0,31 & 0,37 & 0,44 & 0,21 & 0,31 & 0,37 & 0,44 & 0,21 & 0,31 & 0,37 & 0,44 \\
\hline & 8,13 & Jan-99 & Dec-03 & MTAR* & $-0,56$ & 0,07 & 0,55 & 0,24 & 0,28 & 0,34 & 0,41 & 0,24 & 0,28 & 0,34 & 0,41 & 0,24 & 0,28 & 0,34 & 0,41 & 0,24 & 0,28 & 0,34 & 0,41 \\
\hline & 8,14 & Jan-99 & Dec-03 & SYM & & 0,02 & 0,72 & 0,04 & 0,10 & 0,18 & 0,31 & 0,04 & 0,10 & 0,18 & 0,31 & 0,04 & $\begin{array}{l}0,10 \\
0,10\end{array}$ & 0,18 & 0,31 & 0,04 & 0,10 & 0,18 & 0,31 \\
\hline & $\begin{array}{l}8,15 \\
8,15\end{array}$ & Jan-94 & Dec-98 & MTAR* & $-1,42$ & 0,08 & 0,18 & 0,06 & 0,12 & 0,16 & 0,17 & 0,06 & 0,12 & 0,16 & 0,17 & 0,06 & 0,12 & 0,16 & 0,17 & 0,06 & 0,12 & 0,16 & 0,17 \\
\hline & $\begin{array}{l}8,13 \\
8,15\end{array}$ & Jan-95 & Dec-99 & MTAR* & $\begin{array}{l}-1,42 \\
-0,87\end{array}$ & $\begin{array}{l}0,00 \\
0,00\end{array}$ & $\begin{array}{l}0,10 \\
0,23\end{array}$ & $\begin{array}{l}0,00 \\
0,00\end{array}$ & $\begin{array}{l}0,12 \\
0,12\end{array}$ & $\begin{array}{l}0,10 \\
0,20\end{array}$ & 0,23 & $\begin{array}{l}0,00 \\
0,00\end{array}$ & $\begin{array}{l}0,12 \\
0,12\end{array}$ & $\begin{array}{l}0,10 \\
0,20\end{array}$ & 0,23 & $\begin{array}{l}0,00 \\
0,00\end{array}$ & $\begin{array}{l}0,12 \\
0,12\end{array}$ & $\begin{array}{l}0,10 \\
0,20\end{array}$ & 0,23 & $\begin{array}{l}0,00 \\
0,00\end{array}$ & 0,12 & $\begin{array}{l}0,10 \\
0,20\end{array}$ & 0,23 \\
\hline & 8,15 & Jan-96 & Dec-00 & MTAR* & $-0,04$ & $-0,02$ & 0,21 & $-0,03$ & 0,04 & 0,26 & 0,19 & $\begin{array}{r}-0,03 \\
-0,0\end{array}$ & 0,15 & 0,18 & 0,21 & $\begin{array}{r}-0,03 \\
-03\end{array}$ & 0,04 & 0,26 & 0,19 & $-0,03$ & 0,15 & 0,26 & 0,20 \\
\hline & 8,15 & Jan-97 & Dec-01 & MTAR* & $-0,21$ & $-0,02$ & 0,10 & $-0,02$ & 0,01 & 0,05 & 0,08 & $-0,02$ & 0,01 & 0,05 & 0,08 & $-0,02$ & 0,01 & 0,05 & 0,08 & $-0,02$ & 0,01 & 0,05 & 0,08 \\
\hline & 8,15 & Jan-98 & Dec-02 & MTAR* & $-0,33$ & $-0,03$ & 0,17 & $-0,02$ & 0,01 & 0,05 & 0,10 & $-0,02$ & 0,01 & 0,05 & 0,1 & -0, & 0,01 & 0,05 & & -0, & 0,01 & 0,05 & 0,10 \\
\hline & 8,15 & Jan-99 & Dec-03 & MTAR* & 0,05 & $-0,01$ & 0,20 & $-0,01$ & 0,00 & 0,02 & 0,04 & $\begin{array}{l}-0,02 \\
-0,01\end{array}$ & 0,00 & 0,02 & 0,04 & $\begin{array}{l}-0,01 \\
-0,01\end{array}$ & 0,00 & 0,02 & 0,04 & $\begin{array}{l}-0,01 \\
-0,01\end{array}$ & 0,00 & 0,02 & 0,04 \\
\hline Poland & 8,01 & Jan-97 & Dec-01 & SYM & & 0,05 & 0,72 & 0,17 & 0,45 & 0,74 & 0,75 & 0,17 & 0,45 & 0,74 & 0,75 & 0,17 & 0,45 & 0,74 & 0,75 & 0,17 & 0,45 & 0,74 & 0,75 \\
\hline & 8,01 & Jan-98 & Dec-02 & SYM & & 0,17 & 0,84 & 0,35 & 0,63 & 0,82 & 0,84 & 0,35 & 0,63 & 0,82 & 0,84 & 0,35 & 0,63 & 0,82 & 0,84 & 0,35 & 0,63 & 0,82 & 0,84 \\
\hline & $\begin{array}{l}8,01 \\
8,01\end{array}$ & Jan-99 99 & Jun-03 & SYM & & 0,18 & $\begin{array}{l}0,04 \\
0,80\end{array}$ & 0,37 & $\begin{array}{l}0,03 \\
0,64\end{array}$ & $\begin{array}{l}0,82 \\
0,79\end{array}$ & $\begin{array}{l}0,04 \\
0,81\end{array}$ & 0,37 & 0,64 & $\begin{array}{l}0,02 \\
0,79\end{array}$ & $\begin{array}{l}0,84 \\
0,81\end{array}$ & 0,37 & 0,64 & $\begin{array}{l}0,02 \\
0,79\end{array}$ & $\begin{array}{l}0,04 \\
0,81\end{array}$ & 0,37 & 0,64 & 0,02 & $\begin{array}{l}0,04 \\
0,81\end{array}$ \\
\hline & 8,02 & Jan-97 & Dec-01 & SYM & & 0,05 & 0,75 & 0,18 & 0,48 & 0,74 & 0,77 & 0,18 & 0,48 & 0,74 & 0,7 & 0,18 & 0,48 & 0,74 & 0,7 & 0,18 & 0,48 & 0,74 & 0,77 \\
\hline & $\begin{array}{l}8,02 \\
8,02\end{array}$ & Jan-98 & Dec-02 & SYM & & 0,15 & 0,87 & 0,34 & $\begin{array}{l}0,40 \\
0,66\end{array}$ & 0,86 & 0,87 & 0,34 & $\begin{array}{l}0,40 \\
0,66\end{array}$ & 0,86 & 0,87 & 0,34 & $\begin{array}{l}0,40 \\
0,66\end{array}$ & 0,86 & 0,87 & 0,34 & $\begin{array}{l}0,40 \\
0,66\end{array}$ & 0,86 & 0,87 \\
\hline & 8,02 & Jan-99 & Jun-03 & SYM & & 0,18 & 0,83 & 0,36 & 0,64 & 0,81 & 0,83 & 0,36 & 0,64 & 0,81 & 0,83 & 0,36 & 0,64 & 0,81 & 0,83 & 0,36 & 0,64 & 0,81 & 0,83 \\
\hline & 8,03 & Jan-97 & Dec-01 & SYM & & 0,06 & 0,79 & 0,19 & 0,48 & 0,75 & 0,81 & 0,19 & 0,48 & 0,75 & 0,81 & 0,19 & 0,48 & 0,75 & 0,81 & 0,19 & 0,48 & 0,75 & 0,81 \\
\hline & $\begin{array}{l}8,03 \\
8,03\end{array}$ & Jan-98 & Dec-02 & SYM & & 0,17 & 0,88 & 0,19 & $\begin{array}{l}0,40 \\
0,67\end{array}$ & 0,85 & $\begin{array}{l}0,01 \\
0,88\end{array}$ & $\begin{array}{l}0,19 \\
0,37\end{array}$ & $\begin{array}{l}0,40 \\
0,67\end{array}$ & 0,85 & $\begin{array}{l}0,81 \\
0,88\end{array}$ & $\begin{array}{l}0,19 \\
0,37\end{array}$ & $\begin{array}{l}0,40 \\
0,67\end{array}$ & 0,85 & 0, & 0,19 & $\begin{array}{l}0,40 \\
0,67\end{array}$ & 0,85 & $\begin{array}{l}0,01 \\
0,88\end{array}$ \\
\hline & 8,03 & Jan-99 & Jun-03 & SYM & & 0,21 & 0,8 & 0,38 & 0,61 & 0,76 & 0,82 & 0,38 & 0,61 & 0,76 & 0,8 & 0,38 & 0,61 & 0,76 & 0,8 & 0,38 & 0,61 & 0,76 & 0,82 \\
\hline & $\begin{array}{l}8,04 \\
8,04\end{array}$ & Jan-97 & Dec-01 & SYM & & $\begin{array}{l}0,21 \\
0,07\end{array}$ & 0,84 & 0 & 0,49 & 0,76 & $\begin{array}{l}0,02 \\
0,86\end{array}$ & $\begin{array}{l}0,50 \\
0,20\end{array}$ & 0,49 & 0,76 & $\begin{array}{l}0,82 \\
0,86\end{array}$ & $\begin{array}{l}0,50 \\
0,20\end{array}$ & 0,49 & 0,76 & $\begin{array}{l}0,02 \\
0,86\end{array}$ & 0,20 & 0,49 & 0,76 & 0,86 \\
\hline & 8,04 & Jan-98 & Dec-02 & SYM & & 0,17 & 0,90 & 0,37 & 0,66 & 0,86 & 0,90 & 0,37 & 0,66 & 0,86 & 0,90 & 0,37 & 0,66 & 0,86 & 0,90 & 0,37 & 0,66 & 0,86 & 0,90 \\
\hline & 8,04 & Jan-99 & Jun-03 & SYM & & 0,21 & 0,82 & 0,36 & 0,56 & 0,71 & 0,80 & 0,36 & 0,56 & 0,71 & 0,80 & 0,36 & 0,56 & 0,71 & 0,80 & 0,36 & 0,56 & 0,71 & 0,80 \\
\hline & 8,05 & Jan-97 & Dec-01 & SYI & & & 0,8 & 0,22 & 0,49 & 0,71 & 0, & 0,2 & 0,49 & 0 & 0 & 0,2 & 0,4 & 0,7 & & 0,2 & 0,49 & 0,71 & 0,81 \\
\hline & $\begin{array}{l}8,05 \\
8,05\end{array}$ & Jan-98 & Dec-02 & SYM & & 0,16 & 0,89 & 0,35 & 0,63 & 0,81 & 0,89 & 0,35 & 0,63 & 0,8 & 0,8 & 0,35 & 0,63 & 0,81 & 0,89 & 0,35 & 0,63 & 0,81 & 0,89 \\
\hline & 8,05 & Jan-99 & Jun-0 & SYM & & 0,22 & 0,83 & 0,44 & 0,71 & 0,82 & 0,83 & 0,44 & 0,7 & 0,8 & 0, & 0,44 & 0,71 & 0,82 & 0,83 & 0,44 & 0,71 & 0,82 & 0,83 \\
\hline & 8,06 & Jan-97 & Dec-01 & SYM & & 11 & 0,82 & 0,30 & 0,63 & 0,82 & 0,83 & 0,30 & 0,63 & 0,82 & 0,83 & 0,30 & 0,63 & 0,82 & 0,83 & 0,30 & 0,63 & 0,82 & 0,83 \\
\hline & $\begin{array}{l}8,00 \\
8,07\end{array}$ & Jan-97 & Dec-01 & SYM & & 0,11 & 0,74 & 0,26 & $\begin{array}{l}0,03 \\
0,54\end{array}$ & $\begin{array}{l}0,02 \\
0,73\end{array}$ & $\begin{array}{l}0,83 \\
0,75\end{array}$ & $\begin{array}{l}0,26 \\
0,26\end{array}$ & $\begin{array}{l}0,03 \\
0,54\end{array}$ & $\begin{array}{l}0,02 \\
0,73\end{array}$ & $0, \frac{\infty}{0}$ & $\begin{array}{l}0,50 \\
0,26\end{array}$ & 0,54 & 0,02 & 0,75 & 0,26 & 0,54 & 0,73 & 0,75 \\
\hline & 8,07 & Jan-98 & Dec-02 & SYM & & & & 0,43 & & & & & 0,68 & 0,81 & & & & & & & 0,68 & 0,81 & 0,82 \\
\hline & $\begin{array}{l}8,0 \\
8,0\end{array}$ & Jan-99 & Jun. & SYI & & 0,2 & 0, & 0,48 & 0,75 & 0,8 & 0 & 0,4 & 0, & 0, & 0, & 0,4 & 0, & 0,82 & & 0 & 0,75 & 0,82 & 0,79 \\
\hline & 8,08 & Jan-97 & Dec-01 & BTAR* & 0,34 & & 0,7 & 0,24 & 1,55 & 0,32 & 1, & 0,2 & 1,55 & 0,32 & 1, & 0,24 & 1,55 & 0,32 & 1,0 & 0,24 & 1,55 & 0,32 & 1,04 \\
\hline & 8,08 & Jan-98 & Dec-02 & BTAR* & 0,09 & 0,20 & 0,86 & 0,40 & 0,65 & 0,78 & 0,82 & 0,40 & 0,48 & 0,55 & 0,58 & 0,40 & 0,48 & 0,55 & 0,58 & 0,40 & 0,48 & 0,55 & 0,58 \\
\hline & $\begin{array}{l}8,00 \\
8,08\end{array}$ & Jan-99 99 & Jun-03 & BTAR* & 0,18 & $\begin{array}{l}0,20 \\
0,13\end{array}$ & $\begin{array}{l}0,80 \\
0,83\end{array}$ & $\begin{array}{l}0,40 \\
0,15\end{array}$ & $\begin{array}{l}0,03 \\
0,96\end{array}$ & 1,26 & $\begin{array}{l}0,82 \\
0,63\end{array}$ & $\begin{array}{l}0,40 \\
0,15\end{array}$ & $\begin{array}{l}0,40 \\
0,96\end{array}$ & 1,26 & & $\begin{array}{l}0,40 \\
0,15\end{array}$ & & & & & $\begin{array}{l}0,40 \\
0,96\end{array}$ & 1,26 & $\begin{array}{l}0,50 \\
0,63\end{array}$ \\
\hline & 8, & Jan-97 & Dec-0 & SYM & & & 0 & 0,1 & & & 0,8 & 0, & 0,4 & & & & & & & & 0 & 0,70 & 0,80 \\
\hline & $\begin{array}{l}8,09 \\
8,09\end{array}$ & Jan-98 & Dec-02 & SYM & & 0,16 & 0,87 & 0,35 & $\begin{array}{l}0,44 \\
0,63\end{array}$ & 0,82 & $\begin{array}{l}0,80 \\
0,87\end{array}$ & 0,35 & $\begin{array}{l}0,44 \\
0,63\end{array}$ & 0,82 & $\begin{array}{l}0,80 \\
0,87\end{array}$ & 0,35 & $\begin{array}{l}0,44 \\
0,63\end{array}$ & 0,82 & 0,87 & 0,35 & 0,63 & 0,82 & 0,87 \\
\hline & 8,09 & Jan-99 & Jun-03 & SYM & & 0,19 & 0,84 & 0,37 & 0,63 & 0,81 & 0,84 & 0,37 & 0,63 & 0,81 & 0,84 & 0,37 & 0,63 & 0,81 & 0,84 & 0,37 & 0,63 & 0,81 & 0,84 \\
\hline & 8,1 & Jan-97 & Dec-01 & & & & & & & & & & & & & & & & & & & & \\
\hline & 8,1 & Jan-98 & Dec-02 & BTAR* & 0,06 & 0,21 & 0,91 & 0,43 & 0,45 & 0,61 & 0,61 & 0,43 & 0,45 & 0,61 & 0,61 & 0,43 & 0,45 & 0,61 & 0,61 & 0,43 & 0,45 & 0,61 & 0,61 \\
\hline & 8,1 & & Jun-03 & & & & & & & & & & & & & & & & & & & & \\
\hline & 8,11 & Jan-97 & Dec-01 & SYM & & 0,11 & 0,79 & 0,24 & 0,45 & 0,63 & 0,76 & 0,24 & 0,45 & 0,63 & 0,76 & 0,24 & 0,45 & 0,63 & 0,76 & 0,24 & 0,45 & 0,63 & 0,76 \\
\hline & 8,11 & Jan-98 & Dec-02 & SYM & & 0,17 & 0,86 & 0,35 & 0,59 & 0,76 & 0,84 & 0,35 & 0,59 & 0,76 & 0,84 & 0,35 & 0,59 & 0,76 & 0,84 & 0,35 & 0,59 & 0,76 & 0,84 \\
\hline
\end{tabular}


Table B6: 5-year rolling multipliers of the optimal pass-through model

\begin{tabular}{|c|c|c|c|c|c|c|c|c|c|c|c|c|c|c|c|c|c|c|c|c|c|c|c|}
\hline \multirow[b]{2}{*}{ country } & \multirow[b]{2}{*}{ rate } & \multicolumn{2}{|c|}{ sample period } & \multirow{2}{*}{$\begin{array}{r}\text { pass-through } \\
\text { model }\end{array}$} & \multirow{2}{*}{$\begin{array}{l}\text { thres- } \\
\text { hold } \mathrm{m}\end{array}$} & \multirow{2}{*}{\multicolumn{2}{|c|}{$\begin{array}{l}\text { impact long-run } \\
\text { nultiplier multiplier }\end{array}$}} & mult & pleers for & $r+1 \%$ sh & & & ipliers fo & r - $1 \%$ sho & & multtp & Dhers for - & $+0.25 \%$ & Shock & multt1 & pleers for & $-0.25 \% \mathrm{~s}$ & \\
\hline & & start & end & & & & & $1 \mathrm{mth}$ & $3 \mathrm{mths}$ & $6 \mathrm{mths}$ & $12 \mathrm{mths}$ & $1 \mathrm{mth}$ & $3 \mathrm{mths}$ & $6 \mathrm{mths}$ & $12 \mathrm{mths}$ & $1 \mathrm{mth}$ & $3 \mathrm{mths}$ & $6 \mathrm{mths}$ & $12 \mathrm{mths}$ & $1 \mathrm{mth}$ & $3 \mathrm{mths}$ & $6 \mathrm{mths}$ & $12 \mathrm{mths}$ \\
\hline & 8,11 & $\begin{array}{l}\text { Jan-99 } \\
\end{array}$ & $\begin{array}{l}\text { Jun-03 } \\
\end{array}$ & & & 0,27 & 0,82 & 0,53 & 0,81 & 0,85 & 0,82 & 0,53 & 0,81 & 0,85 & 0,82 & 0,53 & 0,81 & 0,85 & 0,82 & 0,53 & 0,81 & 0,85 & 0,82 \\
\hline & 8,12 & Jan-97 & Dec-01 & SYM & & 0,14 & 0,82 & 0,34 & 0,60 & 0,75 & 0,81 & 0,34 & 0,60 & 0,75 & 0,81 & 0,34 & 0,60 & 0,75 & 0,81 & 0,34 & 0,60 & 0,75 & 0,81 \\
\hline Slovak Republic & 8,01 & Jan-95 & Dec-99 & STD & & 0,20 & 0,32 & 0,28 & 0,32 & 0,32 & 0,32 & 0,28 & 0,32 & 0,32 & 0,32 & 0,28 & 0,32 & 0,32 & 0,32 & 0,28 & 0,32 & 0,32 & 0,32 \\
\hline & 8,01 & Jan-96 & Dec-00 & STD & & 0,21 & 0,38 & 0,30 & 0,36 & 0,38 & 0,38 & 0,30 & 0,36 & 0,38 & 0,38 & 0,30 & 0,36 & 0,38 & 0,38 & 0,30 & 0,36 & 0,38 & 0,38 \\
\hline & 8,01 & Jan-97 & Dec-01 & STD & & 0,21 & 0,36 & 0,30 & 0,35 & 0,36 & 0,36 & 0,30 & 0,35 & 0,36 & 0,36 & 0,30 & 0,35 & 0,36 & 0,36 & 0,30 & 0,35 & 0,36 & 0,36 \\
\hline & 8,01 & Jan-98 & Dec-02 & STD & & 0,21 & 0,45 & 0,32 & 0,41 & 0,44 & 0,45 & 0,32 & 0,41 & 0,44 & 0,45 & 0,32 & 0,41 & 0,44 & 0,45 & 0,32 & 0,41 & 0,44 & 0,45 \\
\hline & 8,01 & Jan-99 & Dec-03 & STD & & 0,24 & 0,52 & 0,37 & 0,48 & 0,51 & 0,52 & 0,37 & 0,48 & 0,51 & 0,52 & 0,37 & 0,48 & 0,51 & 0,52 & 0,37 & 0,48 & 0,51 & 0,52 \\
\hline & 8,02 & Jan-95 & Dec-99 & STD & & 0,04 & 0,07 & 0,06 & 0,07 & 0,07 & 0,07 & 0,06 & 0,07 & 0,07 & 0,07 & 0,06 & 0,07 & 0,07 & 0,07 & 0,06 & 0,07 & 0,07 & 0,07 \\
\hline & 8,02 & Jan-96 & Dec-00 & STD & & 0,06 & 0,12 & 0,09 & 0,11 & 0,12 & 0,12 & 0,09 & 0,11 & 0,12 & 0,12 & 0,09 & 0,11 & 0,12 & 0,12 & 0,09 & 0,11 & 0,12 & 0,12 \\
\hline & 8,02 & Jan-97 & Dec-01 & STD & & 0,06 & 0,12 & 0,09 & 0,11 & 0,12 & 0,12 & 0,09 & 0,11 & 0,12 & 0,12 & 0,09 & 0,11 & 0,12 & 0,12 & 0,09 & 0,11 & 0,12 & 0,12 \\
\hline & 8,02 & Jan-98 & Dec-02 & STD & & 0,05 & 0,12 & 0,09 & 0,11 & 0,12 & 0,12 & 0,09 & 0,11 & 0,12 & 0,12 & 0,09 & 0,11 & 0,12 & 0,12 & 0,09 & 0,11 & 0,12 & 0,12 \\
\hline & 8,02 & Jan-99 & Dec-03 & STD & & 0,10 & 0,60 & 0,19 & 0,32 & 0,44 & 0,55 & 0,19 & 0,32 & 0,44 & 0,55 & 0,19 & 0,32 & 0,44 & 0,55 & 0,19 & 0,32 & 0,44 & 0,55 \\
\hline & $\begin{array}{l}8,02 \\
8,03\end{array}$ & Jan-95 & Dec-99 & STD & & 0,03 & 0,03 & 0,03 & 0,03 & 0,03 & 0,03 & 0,03 & 0,03 & 0,03 & 0,03 & 0,03 & 0,03 & 0,03 & 0,03 & 0,03 & 0,03 & 0,03 & 0,03 \\
\hline & 8,03 & Jan-96 & Dec-00 & STD & & 0,04 & 0,05 & 0,04 & 0,05 & 0,05 & 0,05 & 0,04 & 0,05 & 0,05 & 0,05 & 0,04 & 0,05 & 0,05 & 0,05 & 0,04 & 0,05 & 0,05 & 0,05 \\
\hline & 8,03 & Jan-97 & Dec-01 & STD & & 0,04 & 0,06 & 0,05 & 0,06 & 0,06 & 0,06 & 0,05 & 0,06 & 0,06 & 0,06 & 0,05 & 0,06 & 0,06 & 0,06 & 0,05 & 0,06 & 0,06 & 0,06 \\
\hline & 8,03 & Jan-98 & Dec-02 & STD & & 0,04 & 0,05 & 0,05 & 0,05 & 0,05 & 0,05 & 0,05 & 0,05 & 0,05 & 0,05 & 0,05 & 0,05 & 0,05 & 0,05 & 0,05 & 0,05 & 0,05 & 0,05 \\
\hline & 8,03 & Jan-99 & Dec-03 & STD & & 0,15 & 0,33 & 0,23 & 0,30 & 0,33 & 0,33 & 0,23 & 0,30 & 0,33 & 0,33 & 0,23 & 0,30 & 0,33 & 0,33 & 0,23 & 0,30 & 0,33 & 0,33 \\
\hline & $\begin{array}{l}8,03 \\
8,04\end{array}$ & Jan-95 & Dec-99 & $\begin{array}{l}\text { STD } \\
\text { STD }\end{array}$ & & $\begin{array}{r}, 13 \\
-0,01\end{array}$ & $\begin{array}{r}-0,01 \\
-0,03\end{array}$ & $\begin{array}{r},-23 \\
-0,01\end{array}$ & $\begin{array}{r}-0,001 \\
-\end{array}$ & $\begin{array}{r}-0,01 \\
-0,53\end{array}$ & $\begin{array}{r}0,0,01 \\
-0,01\end{array}$ & $\begin{array}{r}, 23 \\
-0,01\end{array}$ & $\begin{array}{r}-0,001 \\
-0,01\end{array}$ & $\begin{array}{r}-0,03 \\
-0,01\end{array}$ & $\begin{array}{l}-0,01 \\
-0,01\end{array}$ & $\begin{array}{r}0,23 \\
-0,01\end{array}$ & $\begin{array}{r}-0,00 \\
-0,01\end{array}$ & $\begin{array}{l}-0,01 \\
-0,01\end{array}$ & $\begin{array}{r}-0,03 \\
-0,01\end{array}$ & $\begin{array}{r}0,01 \\
-0,01\end{array}$ & $\begin{array}{r}-0,01 \\
-0,0\end{array}$ & $\begin{array}{r}-0,01 \\
-0,53\end{array}$ & $\begin{array}{r}-0,01 \\
-0,01\end{array}$ \\
\hline & 8,04 & Jan-96 & Dec-00 & STD & & 0,01 & 0,02 & 0,02 & 0,02 & 0,02 & 0,02 & 0,02 & 0,02 & 0,02 & 0,02 & 0,02 & 0,02 & 0,02 & 0,02 & 0,02 & 0,02 & 0,02 & 0,02 \\
\hline & $\begin{array}{l}8,04 \\
8,04\end{array}$ & Jan-97 & Dec-01 & $\begin{array}{l}\text { STD } \\
\text { ST }\end{array}$ & & 0,01 & 0,02 & 0,02 & 0,02 & 0,02 & 0,02 & 0,02 & 0,02 & 0,02 & 0,02 & 0,02 & 0,02 & $\begin{array}{l}0,02 \\
0,02\end{array}$ & 0,02 & 0,02 & 0,02 & 0,02 & 0,02 \\
\hline & 8,04 & Jan-98 & Dec-02 & STD & & 0,02 & 0,03 & 0,03 & 0,03 & 0,03 & 0,03 & 0,03 & 0,03 & 0,03 & 0,03 & 0,03 & 0,03 & 0,03 & 0,03 & 0,03 & 0,03 & 0,03 & 0,03 \\
\hline & 8,04 & Jan-99 & Dec-03 & STD & & 0,05 & 0,08 & 0,07 & 0,07 & 0,08 & 0,08 & 0,07 & 0,07 & 0,08 & 0,08 & 0,07 & 0,07 & 0,08 & 0,08 & 0,07 & 0,07 & 0,08 & 0,08 \\
\hline & 8,05 & Jan-95 & Dec-99 & STD & & $-0,02$ & $-0,02$ & $-0,02$ & $-0,02$ & $-0,02$ & $-0,02$ & $-0,02$ & $-0,02$ & $-0,02$ & $-0,02$ & $-0,02$ & $-0,02$ & $-0,02$ & $-0,02$ & $-0,02$ & $-0,02$ & $-0,02$ & $-0,02$ \\
\hline & 8,05 & Jan-96 & Dec-00 & STD & & 0,00 & 0,00 & 0,00 & 0,00 & 0,00 & 0,00 & 0,00 & 0,00 & 0,00 & 0,00 & 0,00 & 0,00 & 0,00 & 0,00 & 0,00 & 0,00 & 0,00 & 0,00 \\
\hline & 8,05 & Jan-97 & Dec-01 & STD & & 0,00 & 0,00 & 0,00 & 0,00 & 0,00 & 0,00 & 0,00 & 0,00 & 0,00 & 0,00 & 0,00 & 0,00 & 0,00 & 0,00 & 0,00 & 0,00 & 0,00 & 0,00 \\
\hline & 8,05 & Jan-98 & Dec-02 & STD & & 0,01 & 0,01 & 0,01 & 0,01 & 0,01 & 0,01 & 0,01 & 0,01 & 0,01 & 0,01 & 0,01 & 0,01 & 0,01 & 0,01 & 0,01 & 0,01 & 0,01 & 0,01 \\
\hline & $\begin{array}{l}8,05 \\
8,05\end{array}$ & Jan-99 & Dec-03 & STD & & 0,03 & 0,03 & 0,03 & 0,03 & 0,03 & 0,03 & 0,03 & 0,03 & 0,03 & 0,03 & 0,03 & 0,03 & 0,03 & 0,03 & 0,03 & 0,03 & 0,03 & 0,03 \\
\hline & 8,06 & Jan-95 & Dec-99 & STD & & 0,03 & 0,03 & 0,03 & 0,03 & 0,03 & 0,03 & 0,03 & 0,03 & 0,03 & 0,03 & 0,03 & & & & 0,03 & 0,03 & 0,03 & 0,03 \\
\hline & 8,06 & Jan-96 & Dec-00 & STD & & 0,04 & 0,04 & 0,04 & 0,04 & 0,04 & 0,04 & 0,04 & 0,04 & 0,04 & 0,04 & 0,04 & 0,04 & 0,04 & 0,04 & 0,04 & 0,04 & 0,04 & 0,04 \\
\hline & 8,06 & Jan-97 & Dec-01 & STD & & 0,05 & 0,05 & 0,05 & 0,05 & 0,05 & 0,05 & 0,05 & 0,05 & 0,05 & 0,05 & 0,05 & 0,05 & 0,05 & 0,05 & 0,05 & 0,05 & 0,05 & 0,05 \\
\hline & 8,06 & Jan-98 & Dec-02 & STD & & 0,06 & 0,06 & 0,06 & 0,06 & 0,06 & 0,06 & 0,06 & 0,06 & 0,06 & 0,06 & 0,06 & 0,06 & 0,06 & 0,06 & 0,06 & 0,06 & 0,06 & 0,06 \\
\hline & 8,06 & Jan-99 & Dec-03 & STD & & 0,04 & 0,05 & 0,05 & 0,05 & 0,05 & 0,05 & 0,05 & 0,05 & 0,05 & 0,05 & 0,05 & 0,05 & 0,05 & 0,05 & 0,05 & 0,05 & 0,05 & 0,05 \\
\hline & 8,07 & Jan-95 & Dec-99 & BTAR* & 0,55 & 0,01 & 0,00 & 0,00 & 0,01 & 0,02 & 0,01 & 0,00 & 0,01 & 0,02 & 0,01 & 0,00 & 0,01 & 0,02 & 0,01 & 0,00 & 0,01 & 0,02 & 0,01 \\
\hline & 8,07 & Jan-96 & Dec-00 & & & & & & & & & & & & & & & & & & & & \\
\hline & 8,07 & Jan-97 & Dec-01 & BTAR* & 0,02 & 0,01 & 0,00 & 0,02 & 0,02 & 0,02 & 0,02 & 0,02 & 0,02 & 0,02 & 0,02 & 0,02 & 0,02 & 0,02 & 0,02 & 0,02 & 0,02 & 0,02 & 0,02 \\
\hline & 8,07 & Jan-98 & Dec-02 & & & & & & & & & & & & & & & & & & & & \\
\hline & 8,07 & Jan-99 & Dec-03 & BTAR* & 2,42 & $-0,02$ & $-0,20$ & $-0,02$ & $-0,06$ & $-0,10$ & $-0,15$ & $-0,02$ & $-0,06$ & $-0,10$ & $-0,15$ & $-0,02$ & $-0,06$ & $-0,10$ & $-0,15$ & $-0,02$ & $-0,06$ & $-0,10$ & $-0,15$ \\
\hline Slovenia & 8,01 & Jan-93 & Dec-97 & STD_LL & & 0,69 & 0,63 & 0,63 & 0,63 & 0,63 & 0,63 & 0,63 & 0,63 & 0,63 & 0,63 & 0,63 & 0,63 & 0,63 & 0,63 & 0,63 & 0,63 & 0,63 & 0,63 \\
\hline Sioverita & $\begin{array}{l}8,01 \\
8,01\end{array}$ & Jan-94 & Dec-98 & STD LL & & 0,62 & 0,65 & 0,65 & 0,65 & 0,65 & 0,65 & 0,65 & 0,65 & 0,65 & 0,65 & 0,65 & 0,65 & 0,65 & 0,65 & 0,65 & 0,65 & 0,65 & 0,65 \\
\hline & 8,01 & Jan-95 & Dec-99 & STD_LL & & 0,42 & 0,66 & 0,57 & 0,65 & 0,66 & 0,66 & 0,57 & 0,65 & 0,66 & 0,66 & 0,57 & 0,65 & 0,66 & 0,66 & 0,57 & 0,65 & 0,66 & 0,66 \\
\hline & 8,01 & Jan-96 & Dec-00 & STD LL & & 0,19 & 0,59 & 0,31 & 0,46 & 0,55 & 0,58 & 0,31 & 0,46 & 0,55 & 0,58 & 0,31 & 0,46 & 0,55 & 0,58 & 0,31 & 0,46 & 0,55 & 0,58 \\
\hline & 8,01 & Jan-97 & Dec-01 & D_LL & & & & 0,50 & & 1 , & 1, & 0,5 & 0,8 & 1, & & & & & & & & 1,13 & 1,36 \\
\hline & 8,01 & Jan-98 & Dec-02 & STD_LL & & 0,14 & 0,95 & 0,26 & 0,45 & 0,64 & 0,83 & 0,26 & 0,45 & 0,64 & 0,8 & 0,26 & 0,45 & 0,64 & 0,83 & 0,26 & 0,45 & 0,64 & 0,83 \\
\hline & 8,01 & Jan-99 & Dec-03 & D_LL & & 0 , & 0,38 & 0,10 & & 0,26 & 0 & 0,10 & 0, & 0,26 & 0, & 0,10 & 0,18 & 0,2 & & 0,10 & 0,18 & 0,26 & 0,33 \\
\hline & 8,02 & Jan-93 & Dec-S & STD LL & & 0 , & 0,76 & 0,76 & & 0,76 & 0,76 & 0,76 & 0,76 & 0,76 & 0,7 & 0,76 & 0,76 & 0,76 & 0,7 & 0,76 & 0,76 & 0,76 & 0,76 \\
\hline & $\begin{array}{l}8,02 \\
8,02\end{array}$ & Jan-94 & Dec-98 & STD_LL & & 0,79 & 0,84 & 0,8 & 0,8 & 0,84 & 0,84 & 0,84 & 0,84 & 0,84 & 0,84 & 0,84 & 0,84 & 0,84 & $\begin{array}{l}0,84 \\
0,84\end{array}$ & 0,84 & 0,84 & 0,84 & 0,84 \\
\hline & 8,02 & & Dec-99 & STD_LL & & & & & & & & & & & & & & & & & & 1,05 & 1,05 \\
\hline & $\begin{array}{l}8,02 \\
8,02\end{array}$ & Jan-96 & Dec-0 0 & D_LL & & & 0,9 & 0,5 & & & 0, & 0, & 0,8 & 0 & 0, & 0, & 0, & 0, & & & 30 & 0,90 & 0,93 \\
\hline & 8,02 & Jan-9 & Dec- & STD_LL & & & 1,7 & & & & & 0, & & 1, & 1,7 & 0,9 & 1,3 & 1,6 & & 0,94 & 1,38 & 1,65 & 1,77 \\
\hline & 8,02 & Jan-98 & Dec-02 & STD LL & & 0,28 & 1,19 & 0,50 & 0,79 & 1,01 & 1,16 & 0,50 & 0,79 & 1,01 & 1,16 & 0,50 & 0,79 & 1,01 & 1,16 & 0,50 & 0,79 & 1,01 & 1,16 \\
\hline & $\begin{array}{l}8,02 \\
8,02\end{array}$ & Jan-99 & Dec-03 & STD LL & & $\begin{array}{l}0,20 \\
0,19\end{array}$ & & 0,36 & 0,66 & 1,00 & 1,44 & & & $\begin{array}{l}1,01 \\
1,00\end{array}$ & 1,44 & & & $\begin{array}{l}1,00 \\
1,0\end{array}$ & & & & $\begin{array}{l}1,00 \\
1,00\end{array}$ & $\begin{array}{l}1,10 \\
1,44\end{array}$ \\
\hline & 8,0 & Jan- & Dec- & STD_LL & & & & 0 & & 0,7 & 0 & 0 & 0 & 0 & & & & & & & & 0,78 & 0,78 \\
\hline & 8,03 & Jan- & & D LL & & & & & & & & & 0, & & 0,8 & 0, & 0, & 0,8 & & 0,85 & 0,86 & 0,86 & 0,86 \\
\hline & 8,03 & Jan-95 & Dec-99 & STD_LL & & 0,68 & 1,03 & 0,91 & 1,01 & 1,03 & 1,03 & 0,91 & 1,01 & 1,03 & 1,03 & 0,91 & 1,01 & 1,03 & 1,03 & 0,91 & 1,01 & 1,03 & 1,03 \\
\hline & 8,03 & Jan-96 & Dec-00 & STD LL & & 0,35 & 0,86 & 0,55 & 0,75 & 0,84 & 0,86 & 0,55 & 0,75 & 0,84 & 0,86 & 0,55 & 0,75 & 0,84 & 0,86 & 0,55 & 0,75 & 0,84 & 0,86 \\
\hline & 8,03 & Jan-97 & Dec-01 & STD_LL & & 0,47 & 1,65 & 0,80 & 1,22 & 1,49 & 1,6 & 0,80 & 1,22 & 1,49 & 1, & 0,80 & 1, & & & & 1,22 & 1,49 & 1,63 \\
\hline & 8,0 & & & STD_LL & & & & & & & & & & & & & & & & & & 3 & 1,22 \\
\hline & $\begin{array}{l}8,03 \\
8,03\end{array}$ & Jan-99 & Dec-03 & STD LL & & 0,21 & $\begin{array}{l}1,20 \\
3,35\end{array}$ & 0,40 & 0,75 & 1,20 & $\begin{array}{l}1,89 \\
1,89\end{array}$ & $\begin{array}{l}0,40 \\
0,40\end{array}$ & 0,75 & 1,20 & $\begin{array}{l}1,22 \\
1,89\end{array}$ & $\begin{array}{l}0,40 \\
0,40\end{array}$ & 0,75 & $\begin{array}{l}1,20 \\
1,20\end{array}$ & $\begin{array}{l}1,22 \\
1,89\end{array}$ & 0 & 0,75 & 1,20 & $\begin{array}{l}1,22 \\
1,89\end{array}$ \\
\hline & 8,04 & Jan-93 & Dec-97 & STD LL & & 0,85 & 0,81 & 0,81 & 0,81 & 0,81 & 0,81 & 0,81 & 0,81 & 0,81 & 0,81 & 0,81 & 0,81 & 0,81 & 0,81 & 0,81 & 0,81 & 0,81 & 0,81 \\
\hline & $\begin{array}{l}8,04 \\
8,04\end{array}$ & Jan-94 & Dec-98 & STD_LL & & 0,82 & 0,89 & 0,89 & 0,89 & 0,89 & 0,89 & 0,89 & 0,89 & 0,89 & 0,89 & 0,89 & 0,89 & 0,89 & 0,89 & 0,89 & 0,89 & 0,89 & 0,89 \\
\hline & 8,04 & Jan-95 & Dec-99 & STD LL & & 0,67 & 1,03 & 0,90 & 1,02 & 1,03 & 1,03 & 0,90 & 1,02 & 1,03 & 1,03 & 0,90 & 1,02 & 1,03 & 1,03 & 0,90 & 1,02 & 1,03 & 1,03 \\
\hline
\end{tabular}


Table B6: 5-year rolling multipliers of the optimal pass-through model

\begin{tabular}{|c|c|c|c|c|c|c|c|c|c|c|c|c|c|c|c|c|c|c|c|c|c|c|}
\hline \multirow{2}{*}{ country } & \multirow{3}{*}{$\frac{\text { rate }}{8.04}$} & \multicolumn{2}{|c|}{ sample $\mathrm{p}$} & \multirow{2}{*}{$\begin{array}{r}\text { pass-through } \\
\text { model }\end{array}$} & \multirow{2}{*}{$\begin{array}{c}\text { thres- } \\
\text { hold } \mathrm{m}\end{array}$} & \multirow{2}{*}{$\begin{array}{l}\text { impact long-run } \\
\text { nultiplier multiplier }\end{array}$} & \multicolumn{4}{|c|}{ multipliers for $+1 \%$ shock } & \multicolumn{4}{|c|}{ multipliers for $-1 \%$ shock } & \multicolumn{4}{|c|}{ multipliers for $+0.25 \%$ shock } & \multicolumn{4}{|c|}{ multipliers for $-0.25 \%$ shock } \\
\hline & & start & end & & & & $1 \mathrm{mth}$ & $3 \mathrm{mths}$ & $6 \mathrm{mths}$ & $12 \mathrm{mths}$ & $1 \mathrm{mth}$ & $3 \mathrm{mths}$ & $6 \mathrm{mths}$ & $12 \mathrm{mths}$ & $1 \mathrm{mth}$ & $3 \mathrm{mths}$ & $6 \mathrm{mths}$ & $12 \mathrm{mths}$ & $1 \mathrm{mth}$ & $3 \mathrm{mths}$ & $6 \mathrm{mths}$ & $12 \mathrm{mths}$ \\
\hline & & Jan-96 & Dec-00 & & 0,31 & 0,82 & 0,50 & 0,69 & 0,79 & 0,82 & 0,50 & 0,69 & 0,79 & 0,82 & 0,50 & 0,69 & 0,79 & 0,82 & 0,50 & 0,69 & 0,79 & 0,82 \\
\hline & 8,04 & Jan-97 & Dec-01 & STD_LL & 0,41 & 1,66 & 0,72 & 1,13 & 1,43 & 1,62 & 0,72 & 1,13 & 1,43 & 1,62 & 0,72 & 1,13 & 1,43 & 1,62 & 0,72 & 1,13 & 1,43 & 1,62 \\
\hline & 8,04 & Jan-98 & Dec-02 & STD_LL & 0,25 & 1,34 & 0,45 & 0,75 & 1,02 & 1,25 & 0,45 & 0,75 & 1,02 & 1,25 & 0,45 & 0,75 & 1,02 & 1,25 & 0,45 & 0,75 & 1,02 & 1,25 \\
\hline & 8,04 & Jan-99 & Dec-03 & STD_LL & 0,21 & 4,37 & 0,40 & 0,77 & 1,25 & 2,04 & 0,40 & 0,77 & 1,25 & 2,04 & & & 1,25 & 2,04 & 0,40 & 0,77 & 1,25 & 2,04 \\
\hline & 8,05 & Jan-93 & Dec-97 & STD_LL & 0, & 0,80 & 0,80 & 0,80 & 0,80 & 0,80 & 0,80 & 0,80 & 0,80 & 0,80 & 0,80 & 0,80 & 0,80 & 0,80 & 0,80 & 0,80 & 0,80 & 0,80 \\
\hline & 8,05 & Jan-94 & Dec-98 & STD_LL & 0, & 0,88 & 0,87 & 0,88 & 0,88 & 0,88 & 0,87 & 0,88 & 0,88 & 0,88 & 0,87 & 0,88 & 0,88 & 0,88 & 0,87 & 0,88 & 0,88 & 0,88 \\
\hline & 8,05 & & Dec-99 & STD_LL & & & 0,86 & 0,99 & 1,01 & 1,01 & 0,86 & 0,99 & 1,01 & 1,0 & & 0, & & & 0,86 & 0,99 & 1,01 & 1,01 \\
\hline & 8,05 & Jan-96 & Dec-00 & STD_LL & 0,30 & 0,80 & 0,48 & 0,67 & 0,77 & 0,80 & 0,48 & 0,67 & 0,77 & 0,80 & 0,48 & 0,67 & 0,77 & 0,80 & 0,48 & 0,67 & 0,77 & 0,80 \\
\hline & 8,05 & Jan-97 & Dec-01 & STD_LL & 0,43 & 1,69 & 0,75 & 1,16 & 1,47 & 1,65 & 0,75 & 1,16 & 1,47 & 1,65 & 0,75 & 1,16 & 1,47 & 1,65 & 0,75 & 1,16 & 1,47 & 1,65 \\
\hline & 8,05 & Jan-98 & Dec-02 & STD_LL & 0,28 & 1,38 & 0,51 & 0,83 & 1,10 & 1,31 & 0,51 & 0,83 & 1,10 & 1,31 & 0,51 & 0,83 & 1,10 & 1,31 & 0,51 & 0,83 & 1,10 & 1,31 \\
\hline & 8,05 & Jan-99 & Dec-03 & STD_LL & 0,23 & 4,00 & 0,44 & 0,84 & 1,35 & 2,14 & 0,44 & 0,84 & 1,35 & 2,14 & 0,44 & 0,84 & 1,35 & 2,14 & 0,44 & 0,84 & 1,35 & 2,14 \\
\hline
\end{tabular}

${ }^{1}$ The optimal pass-through model does not converge for this sub-period. The second best pass-through model is chosen for all sub-periods. ${ }^{2}$ In this panel, empty rows indicate that the optimal pass-through model does not converge. Due to the large number of time deposit rates available, the observation has been deleted. 
Table B7: Country, rate, time, and asymmetric patterns in the speed of the pass-through \begin{tabular}{ll}
$\begin{array}{l}\text { Panel A: Regressions using a panel of all } \\
\text { loan and deposit rate multipliers }\end{array}$ & $\begin{array}{l}\text { Panel B: Regre } \\
\text { rate multiplier }\end{array}$ \\
\cline { 1 - 2 } &
\end{tabular}

Panel C: Regressions using a panel of

multipliers deposit rate multipliers

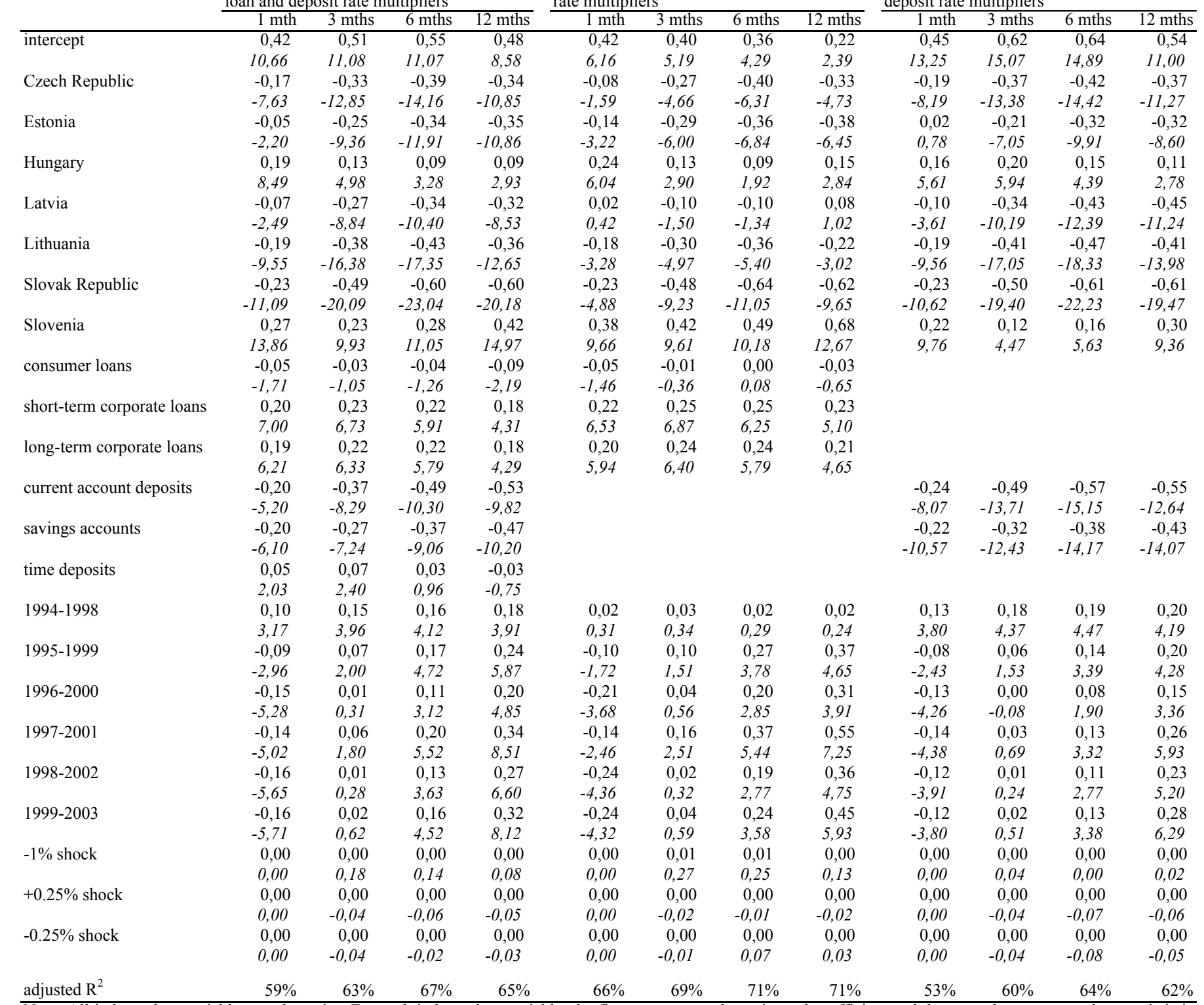

adjusted R

$59 \% \quad 63 \% \quad 67 \% \quad 65 \%$

$66 \% \quad 69 \% \quad 71 \% \quad 71 \%$ 